\title{
All-optical ultrafast switches based on thin film coated- tilted fiber Bragg grating
}

by

Fu Liu

A Thesis submitted to

the Faculty of Graduate Studies and Research in partial fulfillment of the requirements for the degree of

Doctor of Philosophy

Ottawa-Carleton Institute for Electrical and Computer Engineering Department of Electronics

Carleton University

Ottawa, Ontario, Canada

May 2020

Copyright $(\mathrm{C}$ Fu Liu, 2020 


\section{Abstract}

All-optical switching could play a significant role in future high-speed telecommunication networks. Switches based on tilted fiber Bragg gratings (TFBG), coupling the forward propagation core mode to hundreds of discrete backward propagation cladding modes provide a novel platform for light-matter interaction by modulation of material permittivity via the evanescent field of cladding modes which in turn modulate the cladding resonances shifts in wavelength and/or amplitude. In this thesis, in-situ, real time detection of the percolation of Ag films coated on the surface of fibers is achieved through measurements of TFBG transmission spectra by gradually etching the metal films to thicknesses between $15-25 \mathrm{~nm}$. Secondly, all-optical photo-thermal based bistability and self-pulsing, i.e., repetition rate up to $269 \mathrm{~Hz}$ and pulse width of $1.4 \mathrm{~ms}$ was achieved by using a multi-layered graphene-coated TFBG pumped by single Watt-level CW laser light in the fiber core. Thirdly, all-optical synchronous modulation of a probe $\mathrm{CW}$ signal propagating in a gold-coated TFBG immersed in water was achieved by excitation of a plasmonic hybrid cladding guide mode. A $1 \mathrm{MHz}, 25 \mathrm{ps}$ long pump signal with average power of $50 \mathrm{~mW}$ at one wavelength modulated the low power $\mathrm{CW}$ probe at another wavelength by $4.5 \%$, with a widened duration of 56 ps due to hot carrier generation in the gold coating. Finally, broadband antireflection of cladding guided light was demonstrated at the same thicknesses where percolation occurred, which leads to further potential applications in fiber-based nonlinear light-matter interactions. 


\section{Acknowledgments}

I would like to thank and acknowledge first to my supervisor Prof. Jacques Albert, who is super kind, patient like my father to me, and also distinguished, strict on science research. Because of him, I can feel warm in cold days and be strong to face new challenges, and also can enjoy my research project because he opens the door for me to live in a new world where the science research is re-defined.

I would like to acknowledge our professional technician Ms. Albane Laronche for her help with all my experiment. I can't complete my thesis without her. She is so nice and always pass happiness to the people around her. Also thank Mr. Robert Vandusen, Ms. Angela Williams, Mr. Rodney Aiton for their contribution to metal deposition and microscopic imaging, also Dr. Daniel Prezgot, Mr. Mike Bushell from Prof. Anatoli Lanoul's group in Department of Chemistry, Carleton University, for their contribution of performing AFM. Finally, thank Dr. M. Z. Alam from University of Ottawa for his help about my research project.

I would like to thank my friend Pengshuai Ren, Tingting Gang, Xuejun Zhang, Xiaohong Bai for your support and accompany. Thank for my colleague Dr. S. Hamed Jafari, Dr. Salah Abdullah, Dr. Violeta Marquez-cruz, Dr. Wenjun Zhou, Ms. Dingyi Feng, they teach and drive me to be a better researcher. Also thank for Mr. Pierre-Alexis Nault, Mr. Frédéric St-Amand, Ms. Hannah Dinovitzer, they are guiding me to know more about Canada.

Finally, I am sincerely thankful to my family including my parents, brother Hao, sister Jin and my girlfriend Lu, who supported me throughout the years. 


\title{
Preface
}

This thesis is in the form of an "Integrated thesis" for which the bulk of the material, i.e. Chapters 2-4, is a reproduction of three research papers that are either published (Chapter 3-4) or in final revisions (Chapter 2). Therefore, most of the figures for these three chapters are either identical or slightly modified versions of those in the copyrighted articles. Mr. Liu also participated in the research and reporting of several other research articles and refereed conference presentations as a contributing co-author (these additional contributions are not included in this thesis). All these publications, as well as descriptions of the contributions of each of the co-authors, are listed at the end of Chapter 1.

I confirm that $\mathrm{Mr}$. Fu Liu reported on this accurately and in particular that he is the main researcher and writer for the first three papers listed, i.e. Chapters 2-4, of this thesis. For these three chapters, Fu was fully involved in generating research ideas, setting up and conducting the research, obtaining data and analyzing results, as well as preparing and writing the material presented. I also confirm that he is the sole author of the remaining material in the thesis, i.e. Chapters 1,5 and 6.

\author{
Jacques Albert \\ Canada Research Chair in Advanced Photonic Components \\ Associate Editor, IEEE/OSA Journal of Lightwave Technology \\ Fellow of the Optical Society of America \\ Department of Electronics \\ Carleton University
}




\section{Table of Contents}

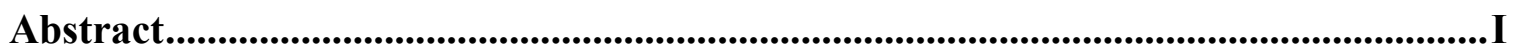

Acknowledgments ....................................................................................................................... II

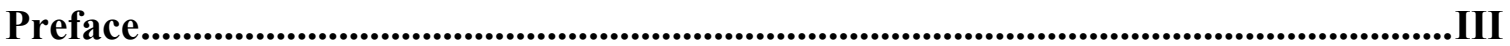

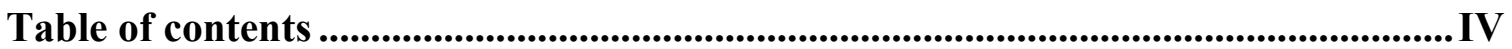

List of Figures................................................................................................................. VII

Nomenclature …................................................................................................................ XVI

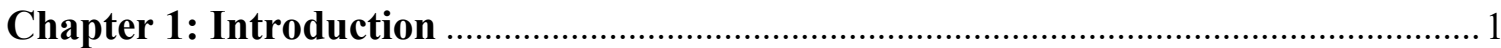

$1.1 \quad$ Tilted fiber Bragg gratings ..........................................................................

1.2 Application of material coated-tilted fiber Bragg gratings ………………………..... 10

1.2.1 Functional material coated-TFBG ………………....................................... 10

1.2.2 TFBG-assisted plasmonics excitation by thin metal film coating ........................ 11

1.2.3 Nanophotonic phenomenon based on metal particle coated-TFBG ……............ 20

1.2.3.1 Nanophotonic phenomenon based on sphere metal particles ……………....2 20

1.2.3.2 Nanophotonic phenomenon based on metal rod ......................................... 25

1.2.3.3 Nanophotonic phenomenon based on random particles at percolation..........29

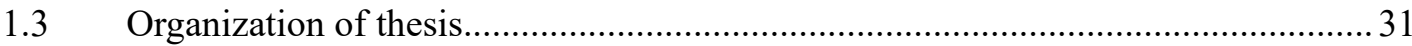

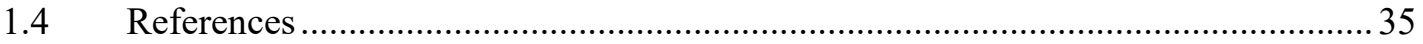

Chapter 2: Optical detection of the percolation threshold of nanoscale silver coatings

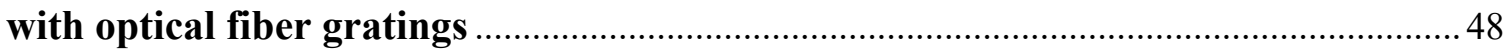

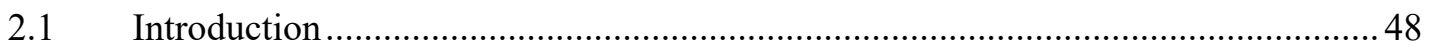

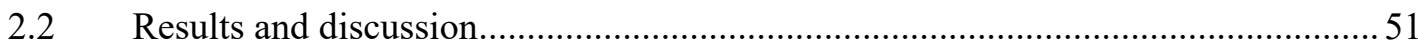

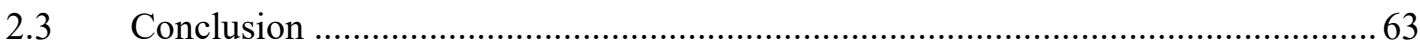

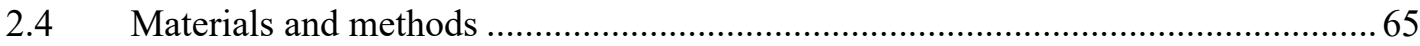

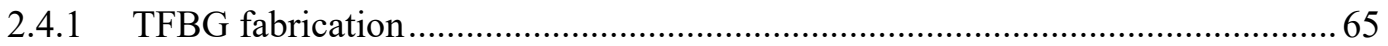




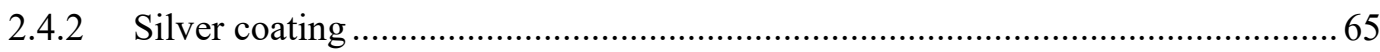

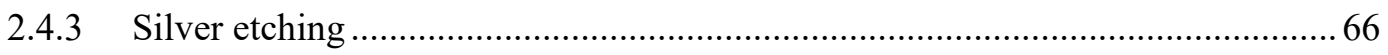

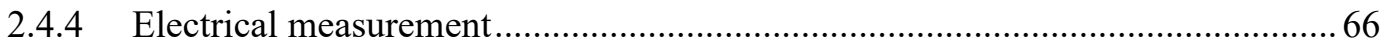

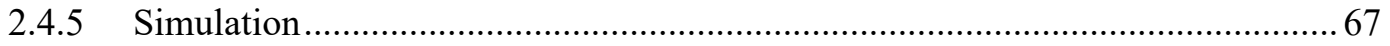

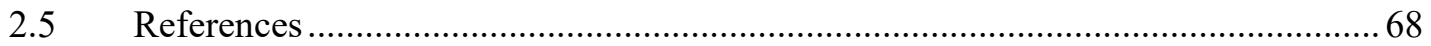

Chapter 3: Saturable absorption and bistable switching of single mode fiber coreguided light by a $6 \mathrm{~nm}$-thick, few layers graphene coating on the cladding surface. 73

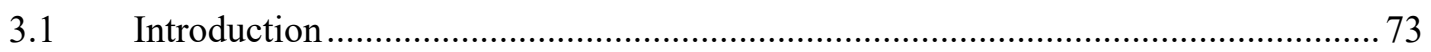

3.2 Photo-thermal effects in graphene coated-TFBG................................................ 76

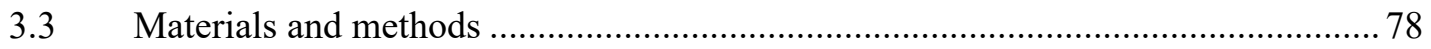

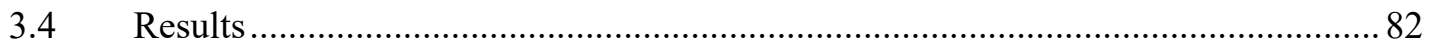

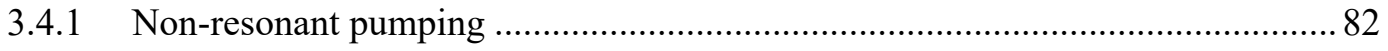

3.2.2 Self-starting modulated switching under resonant pumping ............................... 89

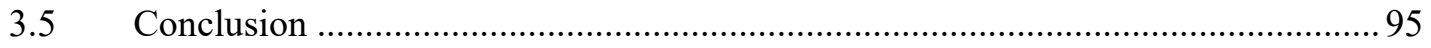

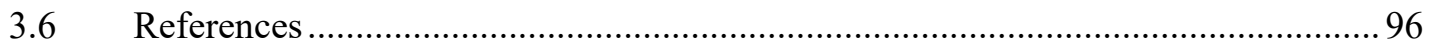

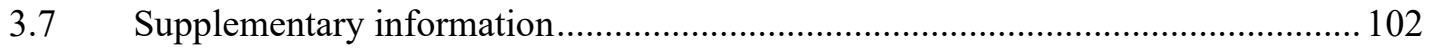

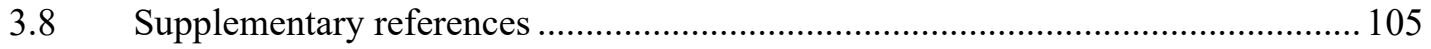

Chapter 4: 40 GHz-rate all-optical cross-modulation of core-guided near infrared light in single mode fiber by surface plasmons on gold-coated tilted fiber Bragg

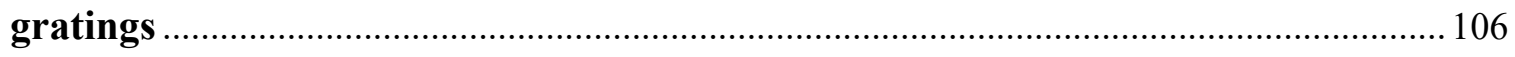

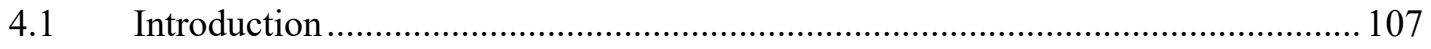

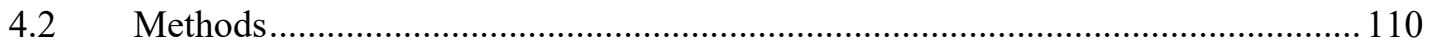

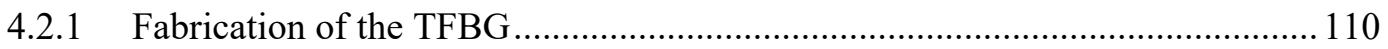

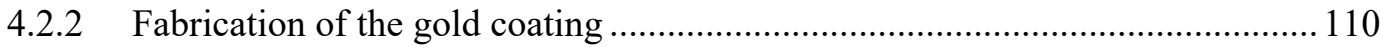

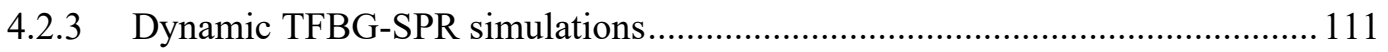

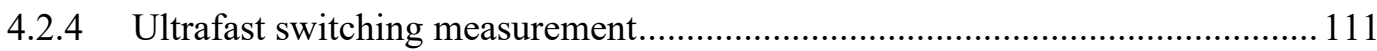




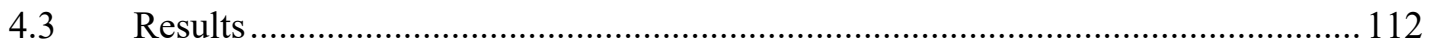

4.3.1 The hybrid SPP-cladding modes of a TFBG …............................................. 112

4.3.2 Ultrafast dynamic of surface plasmon decay ................................................. 117

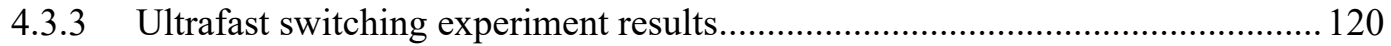

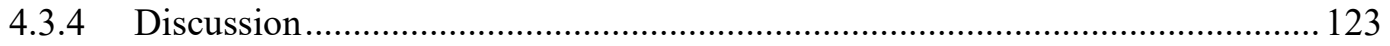

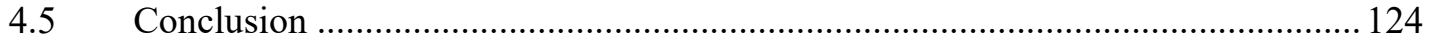

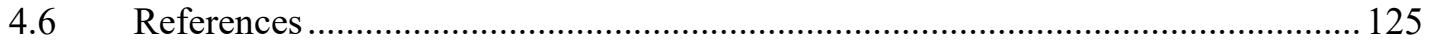

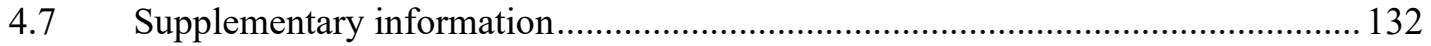

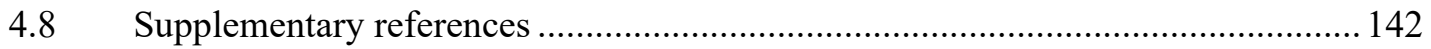

Chapter 5: Broadband anti-reflection coating for fiber cladding modes by partially etched nanoscale silver film at percolation (future work).................................. 144

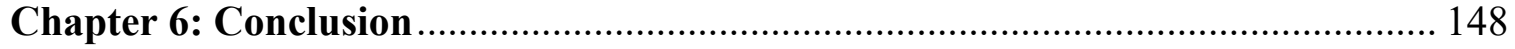




\section{List of Figures}

Fig. 1.1.1. Schematic illustration of the TFBG.......................................2

Fig. 1.1.2. Experimental transmission spectrum of a 12-degree TFBG immersed in water (top); Mode intensity profiles of 5 guided modes near cut-off (bottom) simulated by a complex finite-difference vectoral simulation tool (FIMMWAVE, by Photon Design). The model consisted of a three-layer waveguide: $8.2 \mu \mathrm{m}$ core diameter with refractive index 1.449311, $125 \mu \mathrm{m}$ cladding diameter with refractive index $1.444078,80 \mu \mathrm{m}$ diameter

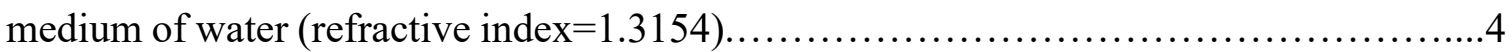

Fig. 1.1.3. Experimental transmission spectra of 12 -degree $T F B G$ in air as a function of the direction of linear polarized incident light........................................

Fig. 1.1.4. Experimental transmission spectra of 12-degree TFBG as a function of the

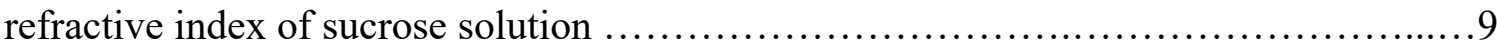

Fig. 1.2.1. (a) Schematic of four waves mixing (FWM) in TFBG coated with single walled carbon nanotubes (SWCNT); (b) Transmission of FWM device pumped by core guided light at $\lambda_{1}$ and $\lambda_{2}$ and producing idler waves at shorter and longer wavelengths (adapted

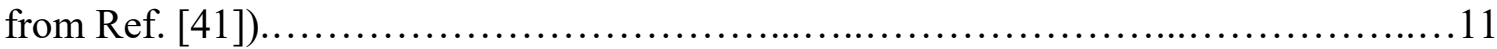

Fig. 1.2.2. Properties of TFBG-SPR: (a) Experimental polarized transmission spectra in water (red, P-pol input with SPP phase matching near $1545 \mathrm{~nm}$, and S-pol input without surface plasmon); (b) Radial (P-pol) and azimuthal (S-pol) dependence of simulated Efield intensity of plasmonic guided mode (the arrows show the E-field vector orientations

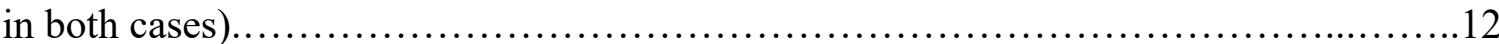


Fig. 1.2.3. Experimental spectra of TFBG-SPR as a function of the refractive index of external dielectric layer. (spectra offset to clarify the linearity of the SPR shift response

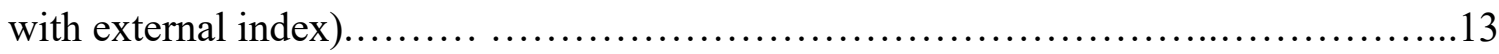

Fig. 1.2.4. Isolated hybridized cladding mode resonances near the SPR as the SRI changes (a), and detailed evolution of one resonance (a,b,c: simulations; d,e:

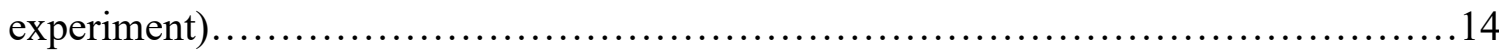

Fig. 1.2.5. (a) Radial distribution of the electric field energy density around the Au layer on the fiber cladding surface for the first adjacent resonance away from the SPR maximum (without the additional dielectric layer); (b) schematic of the Au-coated fiber with an optional additional dielectric "sensing" layer; (c) wavelength shift as a function of the thickness of dielectric layer, for different values of the complex refractive index of the additional layer (indicated by the labels on the plots). The arrows point to the thickness where the wavelength shift reaches $1 / \mathrm{e}$ of its maximum value in each case; (d) resonance transmission spectra corresponding to the situations plotted in (c)

Fig. 1.2.6. Simulated TFBG-SPR Au thickness dependence: (a) P-polarized input core mode; (b) corresponding to the spectrum with Au thickness at $30 \mathrm{~nm}, 50 \mathrm{~nm}$ and $70 \mathrm{~nm}$ of (a); (c) S-polarized input core mode; (d) corresponding to the spectrum with Au thickness at $10 \mathrm{~nm}, 40 \mathrm{~nm}$ and $70 \mathrm{~nm}$. 19

Fig. 1.2.7. The simulated extinction (a), scattering (b) and absorption (c) of Ag particles as a function of radius in water. .23

Fig. 1.2.8. The simulated extinction (a), scattering (b) and absorption (c) of Ag particles at $1550 \mathrm{~nm}$ wavelength. 
Fig. 1.2.9. The simulated absorption of $\mathrm{Ag}$ nanorod as a function of long axis length (the short axis was fixed at $10 \mathrm{~nm}$ ) in water. .26

Fig. 1.2.10. Near-field intensity of LSPR for (a) cylindrical Au nanorod with radius R of $40 \mathrm{~nm}$; (b) a pair of identical cylindrical Au nanorods with $2 \mathrm{~nm}$ gap and $\mathrm{R}=40 \mathrm{~nm}$ (The inset shows the results of two coupled sphere) (reproduced from [82]) .27

Fig. 1.2.11. Scattering cross section for gold nanoparticles embedded in a medium with a large dielectric constant: (a) nanorods, (b) ellipsoidal nanoparticles. The nanoparticle radius is $\mathrm{R}=10 \mathrm{~nm}$, excited by a plane wave polarized along their long axis (reproduced from [83]) 27

Fig. 1.2.12. (a) SEM image of aligned nanowires perpendicular to the fiber axis; (b) the UV-vis-NIR spectrum of the nanowires solution; (c) PDL spectrum of a TFBG coated with randomly oriented nanowires; (d) and with orthogonally aligned nanowires (reproduced

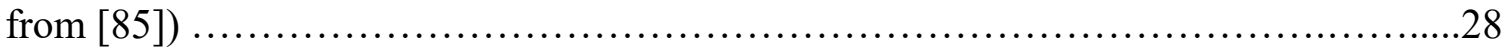

Fig. 1.2.13. Cladding mode response to gold nanoparticle grown through CVD for P- and S- polarized incident input (reproduced from [95]) 30

Fig. 2.1. Sketch of the experimental configuration used to measure transmission of silver coated TFBGs. The device is fully immersed in a dilute etching solution and the input and output leads of the fiber are connected to an external polarization-controlled broadband source (BBS) and optical spectral analyzer (OSA) respectively. For the confirmation of the correlation between the optical measurements and the silver conductivity presented in this work, additional probes from an electrical resistance meter are attached to the silver coating. See Methods for details. .50 
Fig. 2.2. Representative experimental transmission spectra at various characteristic stages of etching for: (a) radially polarized cladding modes; (b) azimuthally polarized cladding modes. The three spectra in each plot are offset for clarity. The green shaded spectral range corresponds to the guided-to-leaky mode transition, or "mode cutoff' .52

Fig. 2.3. AFM of the surface morphology on nominally identical silver coated TFBGs with an initial silver thickness of $50 \mathrm{~nm}$ and etched for various durations (indicated by the labels).

Fig. 2.4. Measured average thickness and roughness from the atomic force microscopy results of Fig. 2.3. A linear fit of the thicknesses between 0 and 230 minutes is used to provide a calibration of the thickness vs etch time correspondence (over this range) for the results of chapter 2 .

Fig. 2.5. Continually measured spectra during etching for the two polarization states, at intervals of 1.5 minutes. Resonances show up as blue lines of various hues except when they diminish significantly in amplitudes, tending towards pink colours. The vertical dashed lines in the figure indicate the approximate mode cutoff point where the mode effective indices become smaller than 1.3154 (the refractive index of water at these

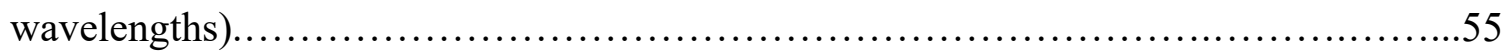

Fig. 2.6. Extraction of the experimental wavelength and amplitude of one of last non-leaky resonances as a function of etch time (the etch time axis is inverted so that the thicknesses

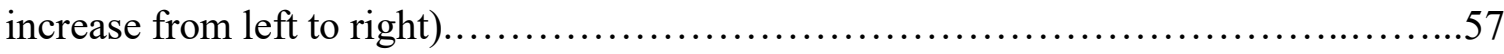

Fig. 2.7. Measurement of the silver coating sheet resistance during etching (filled black squares), with simultaneous measurements of the wavelength shift of the last guided 
resonance (empty red squares) and of its amplitude change (empty blue circles) (the amplitude change is reported here in linear units instead of $\mathrm{dB}$ ). The distance between the

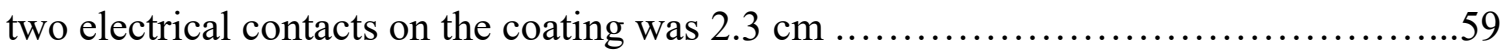

Fig. 2.8. Fitted values of silver refractive index as a function of thickness for thermally evaporated coatings and wavelengths near $1500 \mathrm{~nm}$

Fig. 2.9. Comparison between measured $(a, b)$ and simulated $(c, d)$ wavelength and amplitude shifts for a guided mode near cutoff. The properties of silver used in the simulation are taken from Fig. 2.8 61

Fig. 2.10. Measured transmission spectra of a bare TFBG immersed in media with various refractive indices. The spectra are offset by $\sim 10 \mathrm{~dB}$ for clarity.

Fig. 3.1. Schematic diagram of the G-TFBG. The CW laser input power is coupled out of the core by the cladding mode resonance that in turn modulates the graphene and fiber permittivity by photo-induced Joule heating. The transmission of the $\mathrm{CW}$ laser at the pumping wavelength gets modulated as the grating resonances shift in response to temperature and refractive index change of graphene. Intensity (color bar) and orientation of the electric field of radially and azimuthally polarized cladding modes are also shown................................................................ 76

Fig. 3.2. Transmission spectra of (a) bare; and (b) graphene-coated TFBG ( $1 \mathrm{~cm}$-long, 12 degree tilted), both in air. 80

Fig. 3.3. Microscope images of $12^{\circ}$ TFBG with $514 \mathrm{~nm}$ green light coupled into the core (a1) without graphene on the cladding, and (a2) with graphene; (b) average thickness of graphene from AFM height histograms; AFM images over (c) $5 \mu \mathrm{m} \times 5 \mu \mathrm{m}$, and (d) $2 \mu \mathrm{m}$

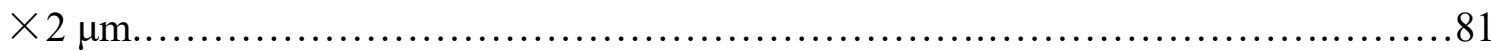


Fig. 3.4. Measured normalized transmission of G-TFBG for several values of input power, and two input core mode polarization: a) P-polarized (radial cladding mode electric field at fiber surface); b) S-polarized (tangential electric field at fiber surface). Note: The actual un-normalized spectra are offset vertically by the change in input power: here, the highest value of the transmitted power was used as the normalization level. Evolution of several S and $\mathrm{P}$ resonance wavelengths (c) and amplitudes (d) as a function of input power in the core mode. .83

Fig. 3.5. Simulation of the electric field of a mode of the graphene coated cladding for (a) S-polarization mode (E field tangent to fiber surface); (b) P-polarization mode (E field radially oriented); (c) mode energy density distribution of the two modes along the fiber diameter. The inset shows the the energy distribution in and around the graphene layer. The overall shapes of the modes in (a) and (b) are essentially identical for the uncoated fiber, while the discontinuity at the cladding surface in the inset of (c) is slightly different

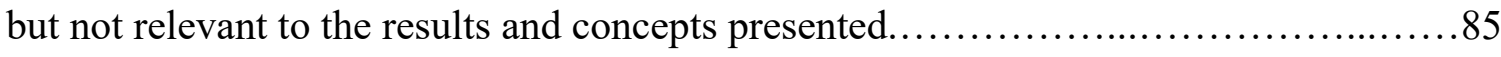

Fig. 3.6. (a) $\mathrm{CW}$ pumping wavelengths used relative to the resonance spectrum (transmission converted from $\mathrm{dB}$ to linear units for better correspondence with parts (bd)); (b) Self-pulsing response for wavelengths shown in (a); (c) Input polarization

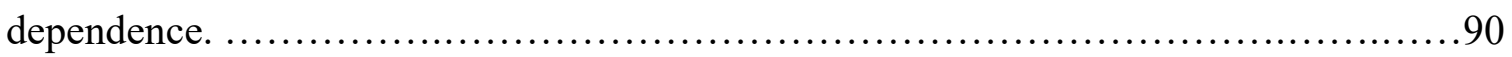

Fig. 3.7. Self-pulsing response to pump power: a) simulation; b) measured ..............92

Fig. 3.8. The self-pulsing repetition rate as a function of pump power in the core .......92

Fig. 3.9. Schematic diagram of the switching system; (b) G-TFBG spectrum changes during non-resonant pumping. The dashed green spectrum represents both the stable output 
power resulting from non-resonant pumping, and the instantaneous one produced by resonant pumping; (c) resonant pumping spectrum.

Fig. 3.10. Simulation of the attenuation of graphene coating-guided cladding modes with effective indices near 1.33 corresponding to $\mathrm{CW}$ pumping position. Each vertical bar corresponds to a particular cladding mode and is color-coded by its polarization state (Red and green for TE and HE modes, which are S-polarized at the cladding surface; Black and blue for TM and EH mode, which are P-polarized)

Fig. 4.1. Schematic diagram of the plasmonic gold-coated TFBG. The pulsed pump $\left(I_{P}\right)$ coupled out of the core by the tilted grating excite SPP on the gold surface and hot carriers $\left(\mathrm{e}^{-}\right.$and $\left.\mathrm{h}^{+}\right)$that in turn modulate the metal permittivity. A co-propagating $\mathrm{CW}$ probe at a different wavelength gets modulated as the grating resonances shift in response to the permittivity change.

Fig. 4.2. TFBG-SPR linear properties: (a) measured spectrum in water (red, SPP phase matching near $1540 \mathrm{~nm}$ ) and air (blue, no surface plasmon at these wavelengths); (b) the Lorentz model fitting for wavelength shifts with- and without- SPR (indicative of changes in the real part of the cladding mode effective index), and of the amplitude change (due to the imaginary part of the mode effective index); (c) The relationship between the Lorentz fit of (b) and the values of the imaginary part of the effective index of guided cladding modes calculated using FIMMWAVE close to SPR (the TM/EH modes are those excited by P-polarized input light). Non SPR-active modes have imaginary parts of the order of $10^{-5}$ and less at the same wavelengths

Fig. 4.3. Plasmonic ultrafast switching based on TFBG spectral combs: (a) simulated spectrum evolution during the hot carrier dynamic process (Supplementary Video 1); (b) 
simulated spectra for electrons at room temperature (black line) and hot electron temperature (870 K, red line); (c) dynamics of cladding modes close to SPR; (d) experimental ultrafast switching of the probe light (Supplementary Video 2)...........118 Fig. 4.4. Pump-induced modulation of $\mathrm{CW}$ probe: (a) the output response with pumping power; (b) the simulation for (a); (c) the output response with probe position; (d) the simulation for (c).

Fig. 4.5. Summary of pump-induced modulation of a $\mathrm{CW}$ probe as a function of the power density in the metal layer. The input (pump) pulse width is $25 \mathrm{ps}$ 122

Fig. 4.6. AFM and SEM images of the Au coating on the fiber cladding. .132

Fig. 4.7. The transient response of $T_{e}$ and $T_{1}$ for hot carriers 134

Fig. 4.8. Evolution of the (a) real and (b) imaginary part of effective RI of the $\mathrm{Au}$ film. 135

Fig. 4.9. (a) Schematic diagram of the switching system using a New Focus $40 \mathrm{GHz}$ bandwidth photodetector ("PD") and a Yenista tunable band blocking filter ("filter") (for measurement of the pump pulse variations, the pump filter is removed); (b) Measured transmission spectrum of the TFBG-SPR 136

Fig. 4.10. The pump pulse response for pumping at different cladding mode wavelengths (see Fig. 4.9 for wavelengths corresponding to mode numbers)

Fig. 4.11. The transmission of pump pulses at mode 3 versus pumping power. 138

Fig. 4.12. Radial dependence of simulated plasmonic guided mode E-fields versus normal guided mode E-field. Insets show corresponding 2D intensity profiles.

Fig. 4.13. Simulated TFBG-SPR Au thickness dependence: (a) P-polarized input core mode; (b) S-polarized. .141 
Fig. 5.1. Transmission spectra of a silver coated TFBG with S-polarized input light at three stages of etching of the silver coating (left) and corresponding AFM images of the silver

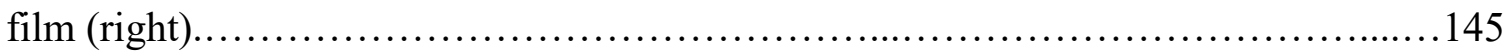

Fig. 5.2. Far-field scattering of metal surface pumping by broadband S-polarized cladding mode immersed in water (a) $50 \mathrm{~nm} \mathrm{Ag} \mathrm{coating;} \mathrm{(b)} \mathrm{Ag} \mathrm{layer} \mathrm{etched} \mathrm{to} \mathrm{antireflection} \mathrm{state;}$

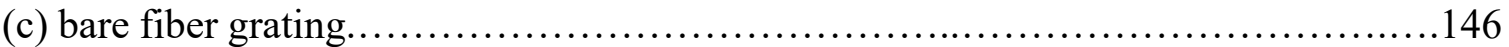




\section{Nomenclature}

$\begin{array}{ll}\text { Symbol } & \text { Description } \\ \lambda & \text { Wavelength } \\ \bar{n}_{11}(\lambda) & \text { Core mode effective index } \\ l & \text { Azimuthal order } \\ m & \text { Radial order } \\ \bar{n}_{l m}(\lambda) & \text { Cladding mode effective index } \\ \Lambda & \text { Grating period } \\ \kappa & \text { Coupling coefficient } \\ \Delta n & \text { Grating induced index perturbation } \\ E_{11} & \text { Transverse electric fields of core mode } \\ E_{l m} & \text { Transverse electric fields of cladding mode } \\ N_{e f f}^{S P P} & \text { Effective index of surface plasmon polariton } \\ \varepsilon_{1} & \text { Permittivity of metal } \\ \varepsilon_{2} & \text { Permittivity of external dielectric } \\ \sigma_{a b s} & \text { Absorption cross section } \\ \sigma_{s c a} & \text { The order of spherical multipole excitation } \\ L & \end{array}$


The surface area of the average particle

The volume of the average particle

Bulk film permittivity of metal

The depolarization factors of nanorod

Temperature perturbation of graphene layer

The power absorbed by graphene layer

Input power in the core of fiber

Fraction of coupling between core mode and cladding mode

Fraction of cladding mode energy in graphene layer

Effective absorption coefficient of graphene layer

The radial coordinate of the fiber

Diffusivity parameter

The length of grating

Propagation constant

Phase mismatch

The lattice heat capacity

The electronic capacity

The temperature of the thermal lattice

The temperature of the thermal electrons

Relaxation time of electron-electron collision

Relaxation time of electron-phonon collision 


$\begin{array}{ll}\omega_{p} & \text { Plasma frequency } \\ k_{B} & \text { Boltzmann constant } \\ \hbar & \text { Reduced Planck constant } \\ \chi_{\text {eff }}(\omega) & \text { Effective linear susceptibility of SPP } \\ m_{\text {eff }} & \text { Effective mass of electrons } \\ \omega_{S P R} & \text { SPR frequency } \\ P_{\text {ave }} & \text { Average power of laser pulse } \\ \tau_{p} & \text { The pulse duration } \\ H & \text { Pulse repetition } \\ S_{m e t a l} & \text { The surface area of gold layer on the fiber cladding } \\ L_{d} & \text { The skin depth of the enhanced plasmonic guide mode } \\ f_{\text {filing }} & \text { Fill factor }\end{array}$

Acronyms

Description

TFBG

Tilted fiber Bragg grating

G-TFBG Graphene coated TFBG

TE

Transverse electric

TM

Transverse magnetic

HE

Hybrid electric mode

EH

Hybrid magnetic mode 


\begin{tabular}{|c|c|}
\hline PDL & Polarization dependent loss \\
\hline SRI & Surrounding refractive index \\
\hline RI & Refractive index \\
\hline RIU & Refractive index unit \\
\hline SPP & Surface Plasmon polariton \\
\hline SPR & Surface Plasmon resonance \\
\hline LSPP & Localized surface Plasmon polariton \\
\hline SEM & Scanning electron microscopy \\
\hline AFM & Atomic force microscopy \\
\hline BBS & Broadband source \\
\hline ASE & Erbium-doped fiber amplified emission source \\
\hline OSA & Optical spectrum analyzer \\
\hline $\mathrm{PC}$ & Polarization controller \\
\hline PD & Power detector \\
\hline SWCNT & Single-walled carbon nanotube \\
\hline FWM & Four waves mixing \\
\hline $\mathrm{CW}$ & Continuous wave \\
\hline APCVD & Atmospheric pressure chemical vapor deposition \\
\hline
\end{tabular}




\section{Chapter 1: Introduction}

Integrated-optical switching devices are considered as significant elements in high speed telecommunication networks today [1]. Unlike the conventional electrically-controlled optical switches with low switching speed, massive all-optical switches with ultrafast responses using nonlinear materials are based on ultrafast modifications of the refractive index of nonlinear media due to Kerr effect or two-photon effects in optical fibers or optical planar waveguides with small cross-section and long nonlinear interaction lengths. Examples include Mach-Zehnder interferometers [2-4], Sagnac interferometers [5-7], optical couplers [8-11], Fabry-Perot resonators [12-14] and ring resonators [15-17]. Meanwhile, the applications of nanophononics technology using materials at nanoscale dimensions, either inorganic (semiconductors, dielectrics, metal, etc.) or organic (molecules, clusters) and biological (cells, proteins, etc...), or nano-structured materials (nano-thin film, nanoparticles, nanowires, nanotubes, etc.) are increasing. These advances in switching systems benefit from a cut down of the threshold power for switching in nanostructure devices like nano-waveguide interferometers [18,19], photonic crystals [20-23], and surface plasmon polaritons devices [24-26]. In contrast with the various configurations so far, a simple, monolithic, low insertion loss, low cost all optical switching structure is proposed in this thesis: the tilted fiber Bragg grating (TFBG) inscribed in standard telecom single mode fiber without physical modification such as mechanically polished D-shape fiber [27,28], unclad or etched fiber taper [29,30], fiber end face coating [31], or microstructure fiber [32-34]. Based on the phase matching between the effective refractive index of the core more, the cladding mode and the grating period, a TFBG couples the 
forward propagating light in the core to backward propagatiing cladding modes (i.e. hundreds of discrete cladding mode resonance separated by $0.2-2 \mathrm{~nm}$ and with a high $\mathrm{Q}$ factor (wavelength/full width at half maximum, larger than $10^{4}$ ). These narrowband transmission loss resonances respond to external permittivity perturbations through the evanescent field of cladding modes and their wavelength shifts or change in amplitude. This has been used to develop high performance sensors for biomedicine [35-37], magnetic detection [38], refractometry based on plasmon excitation [39], or for optical fiber grating based devices with enhanced nonlinear optical properties [40-42]. Therefore, the TFBG is a promising platform for light-interactions targeted for ultrafast switching with simplified structure, low intrinsic loss, ease of fabrication (i.e. in contrast to using an interferometric arrangement with high coherent laser, a phase mask with fixed period (our method) is used to generate the interference pattern so that it is easy for mass-produced TFBG with low coherence ultraviolet sources) and low-cost fiber-coupled instrumentation.

In this chapter, a literature review and description of TFBGs is presented in terms of basic theory, their configurations as sensors, and as a promising platform for light-matter interactions based on nonlinear material coatings. This forms the inspiration for the rest of the work presented in this thesis about all optical switching devices.

\subsection{Tilted fiber Bragg gratings}

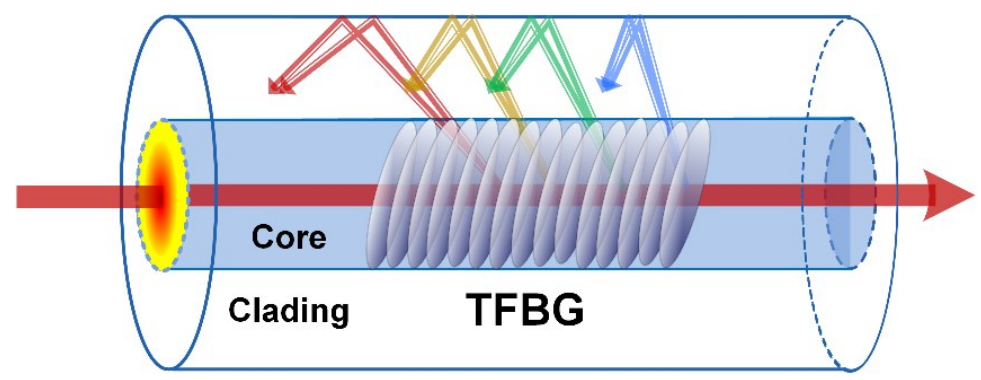

Fig. 1.1.1. Schematic illustration of the TFBG 
The discovery of the formation of gratings in optical fibers has opened up a whole new class of light processing devices, which has developed into a mature technology with a wide range of applications in various areas, including physical sensing for temperature, strain, acoustic waves, pressure and specially nonlinear optical switching components [4345]. Meanwhile, another category of short period FBGs were also proposed that involved tilting the grating planes relative to fiber axis, named TFBG, as shown in Fig. 1.1.1. TFBGs allow the application single mode fibers to surrounding refractive index (SRI) sensing, and all other sensing modalities based on SRI, without modifying the geometry of fiber. This is because TFBGs couple the light from the forward-propagating core-guided mode to a large number of backward-propagating cladding modes that have an evanescent field penetrating in the surrounding medium.

Fig. 1.1.2 shows the experimental transmission spectrum and simulated guided modes (i. e. first 5 guided modes near cutoff) of a 12-degree, $2 \mathrm{~cm}$-length TFBG immersed in water. The core mode resonance (usually referred to as the "Bragg resonance") is located at the longest wavelength around $1610 \mathrm{~nm}$, as decided by the grating period and effective index of the fiber core. A subset of large number of cladding modes guided by the cladding are shown on the left of the Bragg resonance including a "ghost" mode consisting of superposition of several low order cladding modes [46], and high order cladding modes at shorter wavelength. The leaky modes of the cladding, i. e. cladding modes with effective indices lower than the water, are shown beyond $1530 \mathrm{~nm}$ where there is a discontinuity in the cladding mode envelope after which the resonance amplitude decrease sharply. For the whole thesis, we focus on the high order guided cladding modes that obey the phase matching condition with the fundamental $H E_{11}$ mode of the core: 


$$
\lambda=\left(\bar{n}_{11}(\lambda)+\bar{n}_{l m}(\lambda)\right) \Lambda
$$

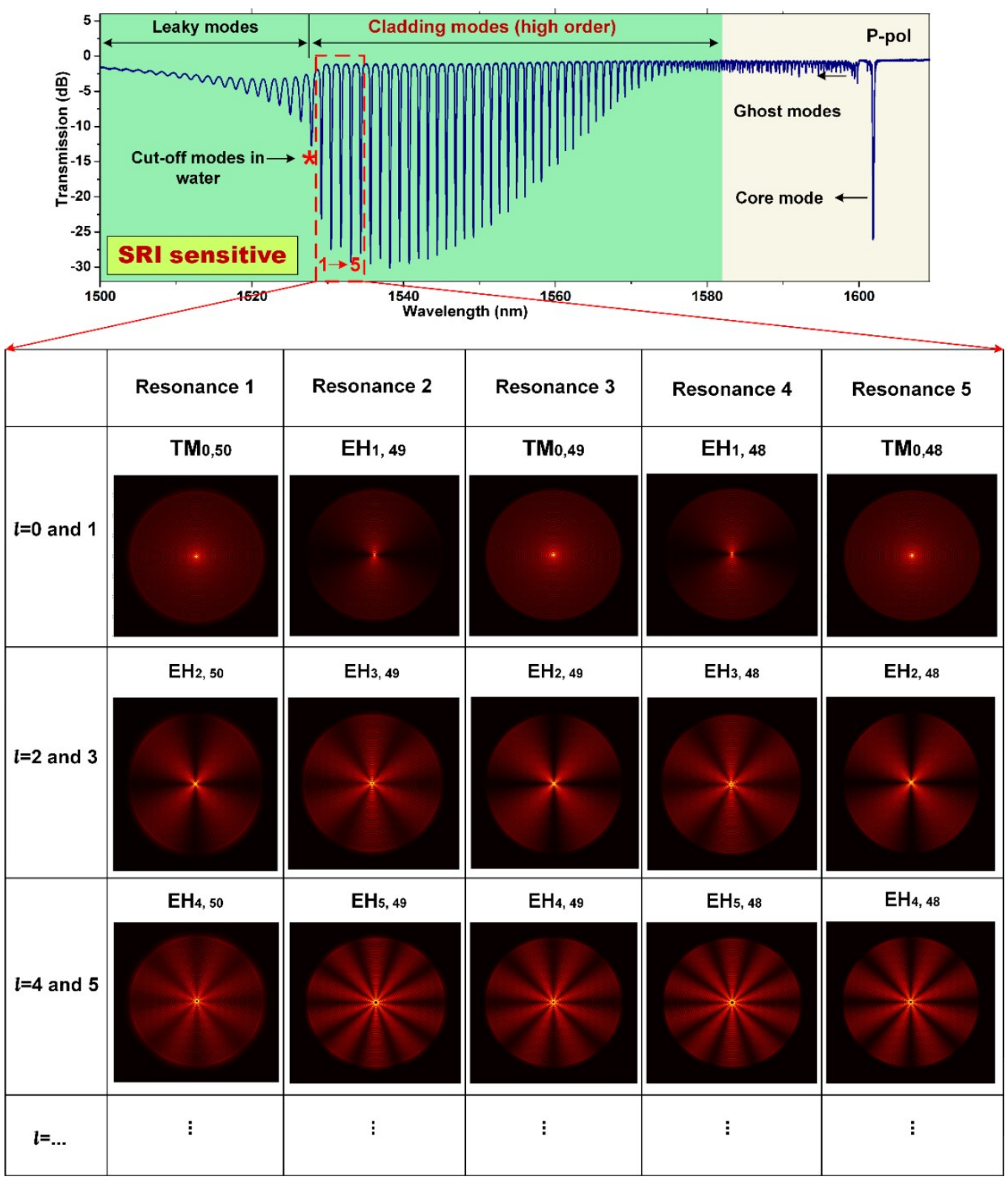

Fig. 1.1.2. Experimental transmission spectrum of a 12-degree TFBG immersed in water (top); Mode intensity profiles of 5 guided modes near cut-off (bottom) simulated by a complex finitedifference vectoral simulation tool (FIMMWAVE, by Photon Design). The model consisted of a three-layer waveguide: $8.2 \mu \mathrm{m}$ core diameter with refractive index $1.449311,125 \mu \mathrm{m}$ cladding diameter with refractive index 1.444078, $80 \mu \mathrm{m}$ diameter medium of water (refractive index=1.3154). 
Where $\lambda$ is the cladding mode resonance wavelength, $\bar{n}_{11}(\lambda)$ is the effective index of core mode, and $\bar{n}_{l m}(\lambda)$ is the effective index of cladding mode (with azimuthal order $l$ and radial order $m$ ). $\Lambda$ is the period of the grating along the fiber axis.

The guided cladding modes in standard single mode fiber consist of four types: $T E_{0 m}$ , $T M_{0 m}, H E_{l m}$ and $E H_{l m}$ based on three layer cylindrical waveguide using analytical methods developed in [47]. Again, $l$ is the azimuthal order and $m$ is the radial order, in which the radial and the azimuthal electric fields are zero for $T E_{0 m}$ and $T M_{0 m}$ modes respectively, while hybrid $H E_{l m}$ and $E H_{l m}$ modes have no vanishing components. In particular, TE modes have their electric fields parallel to the cladding surface while TM modes have their electric fields in the $(r, z)$ plane $(\mathrm{z}$ is the axis of the fiber, and $\mathrm{r}$ the radial coordinate). On the other hand, EH and HE modes have no vanishing field component but it has been demonstrated that for high order modes, the electric field of EH modes is predominantly radially polarized while HE modes are mostly azimuthally polarized [48]. Furthermore, the field patterns of all mode families can be rotated around the fiber axis by an arbitrary angle since the fiber has total cylindrical symmetry (this is meaningless for TE and TM modes since they have no azimuthal dependence). In the following, the polarization state of the four types of cladding modes are separated into two groups according to the electric field orientation: azimuthally polarized modes (TE and HE mode) and radially polarized modes (TM and EH mode).

As for the uniform grating with refractive index modulation $\Delta n$ in the core, the coupling coefficient $(\kappa)$ between the fundamental mode (core mode) and multiple 
cladding modes is calculated by using coupled mode theory based on the weak coupling approximation (the maximum core index modulation is of the order of 0.001 ):

$$
\kappa=\frac{\pi \bar{n}_{c o}}{4 \lambda Z_{0}} \int_{0}^{2 \pi} \int_{0}^{a} E_{11}^{*} E_{l m}\left[\Delta n \cos \left(\frac{2 \pi}{\Lambda} r \cos \varphi \tan \theta\right)\right] r d r d \varphi
$$

where $\bar{n}_{c o}$ is the effective index of the core mode, $Z_{0}=377 \Omega$ is the free space impedance, $E_{11}$ and $E_{l m}$ are the transverse electric fields of the core mode and cladding mode. The magnitude of the coupling coefficient depends on the scalar product of the mode fields of the $E_{11}$ and $E_{l m}$ modes, multiplied by the projection of the tilted grating planes in the cross-section of the core (the refractive index modulation is limited to the core in our case). While the electric field of the fundamental mode of the core is largely linearly polarized (the core is "weakly guiding"), any arbitrary light signal in the core can be decomposed as a linear combination of two orthogonally polarized modes. It is convenient to define the radial direction that lies in the tilt plane as one of the arbitrary orthogonal axes of the fiber cross section, and then the core mode component with an electric field that lies in the tilt plane is "P-polarized" while the orthogonal component is "S-polarized" relative to the tilt plane.

Now orientation of the tilt plane breaks the cylindrical symmetry of the fiber and the result of the integral in Equation (1.2) depends on the orientation of the electric field of the incoming core mode. As demonstrated in Ref. 48 (and proven experimentally countless times in follow up publications from our group), an S-polarized core modes couples primarily to the TE and $H E_{l m}$ modes while a P-polarized core mode couples to TM and $E H_{l m}$ cladding modes. Therefore, if the input core mode is linearly polarized (with an inline polarizer or polarization controller) upstream of the TFBG in the S or P plane, the 
transmission spectrum will only contain $\mathrm{TE} / \mathrm{HE}$ modes or $\mathrm{TM} / \mathrm{EH}$ modes respectively. In the remainder of this thesis, "S" and "P" labels for modes and measurements refer to this convention. Moreover, for both P- and S-polarized cases, cladding modes with odd azimuthal order $(l=1,3,5 \cdots)$ and even azimuthal order $(l=0,2,4 \cdots)$ are nearly degenerate and form alternating groups of resonance pairs in transmission spectra, as shown in bottom of Fig. 1.1.2. It was further demonstrated in Ref. 48 that for gratings with tilt angles of the order of 10 degrees (the maximum tilt used in this thesis) modes with azimuthal orders greater than 9 contribute very little to any resonance. What is important here is that for high order modes (at wavelengths farthest from the Bragg resonance) $\mathrm{P}$ spectra will have only cladding modes with electric fields radially polarized while S spectra will only have modes with electric fields parallel to the cladding surface, with important consequences when cladding mode resonances are used for accurate refractometry [49].
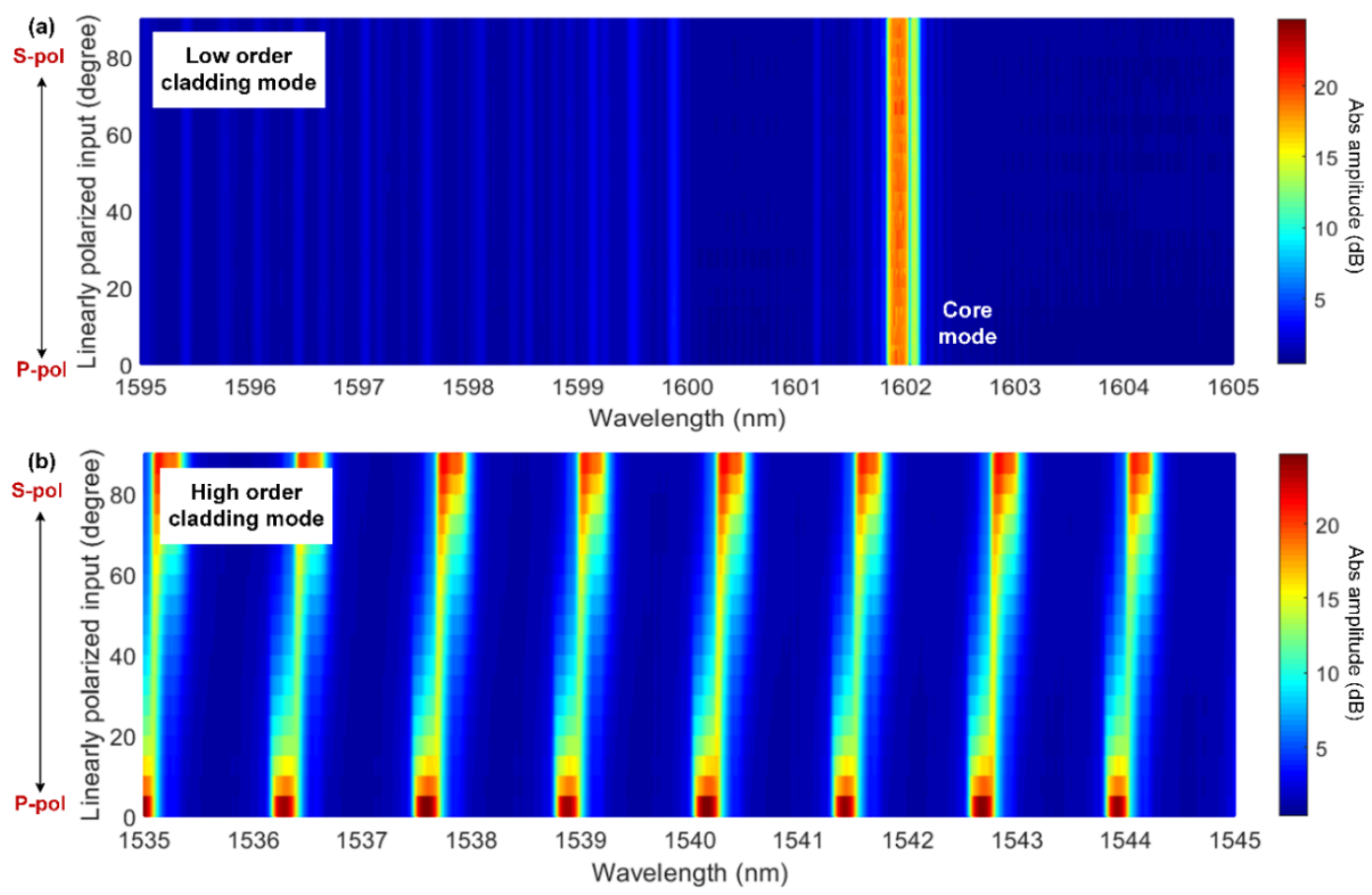

Fig. 1.1.3. Experimental transmission spectra of 12-degree $T F B G$ in air as a function of the direction of linearly polarized incident light. 
Fig. 1.1.3 shows how the experimental transmission spectrum of a TFBG depends on the input core mode polarization direction. The low order cladding modes close to Bragg resonance (small $m$, higher effective indices and in longer resonance wavelengths) have truly mixed polarization states and no visible shift with input polarization (Fig. 1.1.3 (a)). On the other hand, for the high order cladding mode far away from Bragg resonance (large $m$, lower effective indices), shown in Fig. 1.1.3 (b), the cladding mode resonances shift by about one full width at half maximum as the input polarization rotates by 90 degrees. This comes from two effects: 1) as indicated earlier, higher order modes split into TE/HE and $\mathrm{TM} / \mathrm{EH}$ groups and; 2) the boundary condition for the two groups are markedly different because total internal reflection is predominanly TE-like or TM-like, respectively, with correspondingly different phase shifts in the Fresnel reflection coefficients. Therefore, in addition to the alternation between resonances with even $(\mathrm{m}=0,2,4, \ldots)$ and odd $(\mathrm{m}=1,3,5, \ldots)$ azimuthal orders, separated by about $1.3 \mathrm{~nm}$ in Fig. 1.1.3(b), each of these resonances can be further split in two, separated by about $0.1 \mathrm{~nm}$, by polarizing the input core mode along the S or P plane of the TFBG. Finally, it was determined both theoretically and experimentally that in the small polarization splitting of each resonance the short wavelength state of the resonance corresponds to the P-polarized input while the orientation giving rise to the long wavelength state corresponds to the S-polarized input orientation (as indicated by the labels on Fig. 1.1.3). This is very important information in particular for experiments involving metal coatings for plasmonics, because surface plasmon polariton (SPP) waves can only exist in TM states, i.e. with radially polarized electrical fields for metal coatings on optical fibers [50]. 


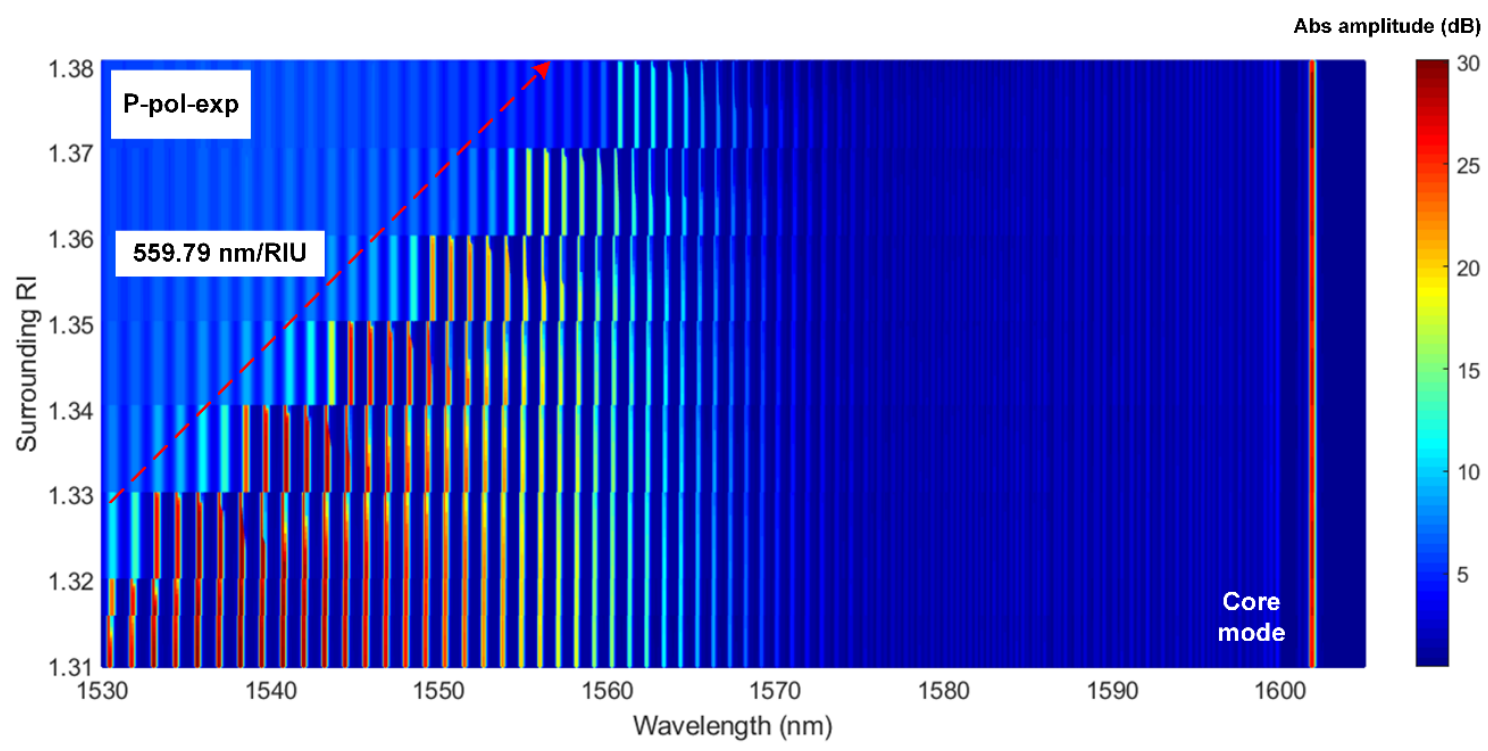

Fig. 1.1.4. Experimental transmission spectra of 12-degree TFBG as a function of the refractive index of sucrose solution.

In order to demonstrate the general use of TFBG devices for sensing, Fig. 1.1.4 shows P transmission spectra of TFBGs immersed in sucrose solution with SRI ranging from 1.31 to 1.38. Since the evanescent field penetration of cladding modes increases with decreasing effective index, the high order cladding modes at shorter wavelengths shift notably more than the lower order modes since a larger fraction of the mode power actually propagates in the external medium. It is also confirmed that the core mode is totally immune to SRI and thus can be used as a power and wavelength reference. Finally, there is a clear cut-off where cladding mode resonances decrease sharply in amplitude along a diagonal in the wavelength/SRI plane, indicating loss of total internal reflection at the point where the cladding mode effective index becomes equal to the SRI. These features allow the construction of all-fiber refractometers either by following the cutoff point (with an uncertainty corresponding to the distance beween individual resonances), as shown in [51] or more accurately by following the exact displacements of the individual resonances [49]. 
Therefore, the thrilling properties of TFBG responding to external environments through the evanescent fields of cladding modes makes it possible to build a platform for lightmatter interaction where the functional material such as organic material, nonlinear material, nanoparticles, semiconductor layer can be well integrated with fiber grating by using the mature coating technologies.

\subsection{Applications based on material coated-TFBG}

\subsubsection{Functional material coated-TFBG}

The functional material coated TFBGs are well studied due to the evanescent field of cladding mode going through and interacting with the coating layer together with the high Q-factor of the cladding mode resonances. Firstly, it is working beautifully as all-optical sensors taking advantage of the simplified, low cost and low insertion loss structure, such as a $\mathrm{pH}$ sensor based on a polyaniline coated-TFBG reacting to $\mathrm{pH}$ in the range of 2-12 with sensitivity of $82 \mathrm{pm} / \mathrm{pH}[52,53]$, an anemometer based on single-walled carbon nanotube (SWCNT) coating because of its outstanding thermal properties with $93 \%$ absorption efficiency and heating the fiber to $146.1{ }^{\circ} \mathrm{C}$ [54], and a RI sensor with high efficiency for the low-refractive index region in a graphene-coated TFBG [55].

Secondly, a TFBG working as a light-matter interaction platform was demonstrated by: 1) Ultrafast nonlinear effects with picosecond time scale obtained by means of pumpprobe experiments based on Kerr effect in carbon nanotubes coatings [40]; and 2) fourwave mixing (FWM) in SWCNT-coated TFBGs with nonlinear coefficient as high as $1.8 \times 10^{3} \mathrm{~W}^{-1} \mathrm{Km}^{-1}$ as shown in Fig. 1.2.1 [41]. These prior investigations by former members of our group indicate promise to achieve more new applications by coating high quality functional materials on the surface of TFBG. 


\section{(a)}

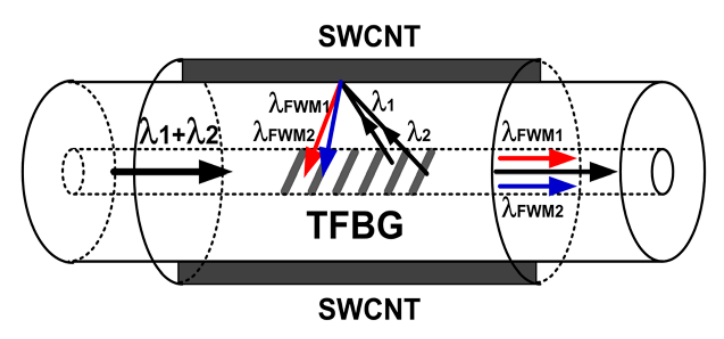

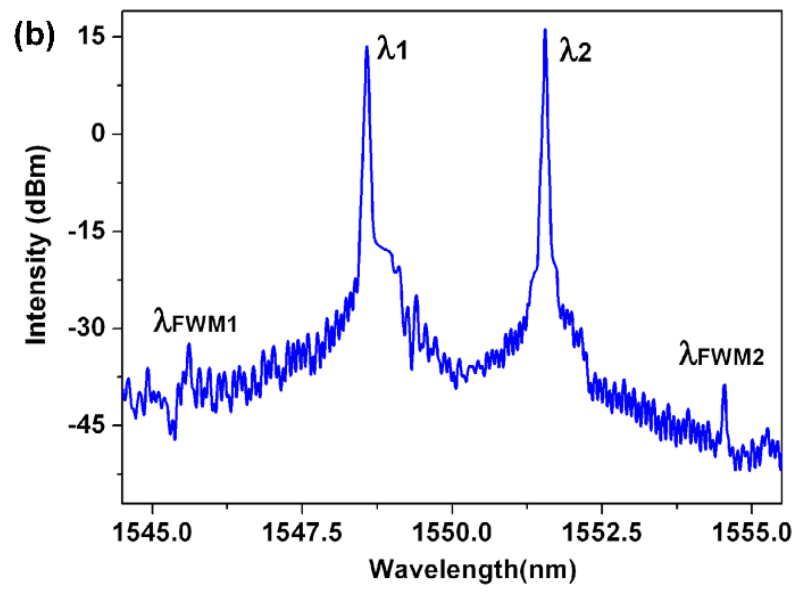

Fig. 1.2.1. (a) Schematic of four waves mixing (FWM) in TFBG coated with single walled carbon nanotubes (SWCNT); (b) Transmission of FWM device pumped by core guided light at $\lambda_{1}$ and $\lambda_{2}$ and producing idler waves at shorter and longer wavelengths (adapted from Ref. [41]).

\subsubsection{TFBG-assisted plasmonics excitation by thin metal film coating}

The surface plasmon polariton (SPP) is an electromagnetic wave that propagates at the boundary between a metal and a dielectric. In a metal coated fiber, hybrid guided SPPcladdings mode can be excited successfully by a TFBG when the effective index of the cladding mode is phase matched to that of the SPP [39], expressed by:

$$
N_{e f f}^{S P P}=\sqrt{\frac{\varepsilon_{1} \varepsilon_{2}}{\varepsilon_{1}+\varepsilon_{2}}}
$$

Where $\varepsilon_{1}, \varepsilon_{2}$ are the relative permittivities of the metal and external dielectric, respectively. For instance, the $N_{e f f}^{S P P}=1.328$ if we consider Au and water at a wavelength of $1550 \mathrm{~nm}$ wavelength and a cladding mode with such effective index will be hybridized with the SPP at a wavelength determined by the period of the TFBG. In the transmission spectra shown in Fig. 1.2.2 (a) (2 cm length, $556.015 \mathrm{~nm}$ period, 12 degree TFBG with 45 $\mathrm{nm}$ of $\mathrm{Au}$ on the cladding surface), the amplitudes of cladding modes near $1545 \mathrm{~nm}$ 
attenuate and "disappear" close to SPP phase matched wavelength with P-polarized incident light where radially polarized cladding modes (i. e. TM-like) are excited. There is no SPP excitation for azimuthally polarized cladding modes (i.e, TE-like). More details of the plasmonic hybrid guided cladding mode fields are shown in Fig. 1.2.2 (b).
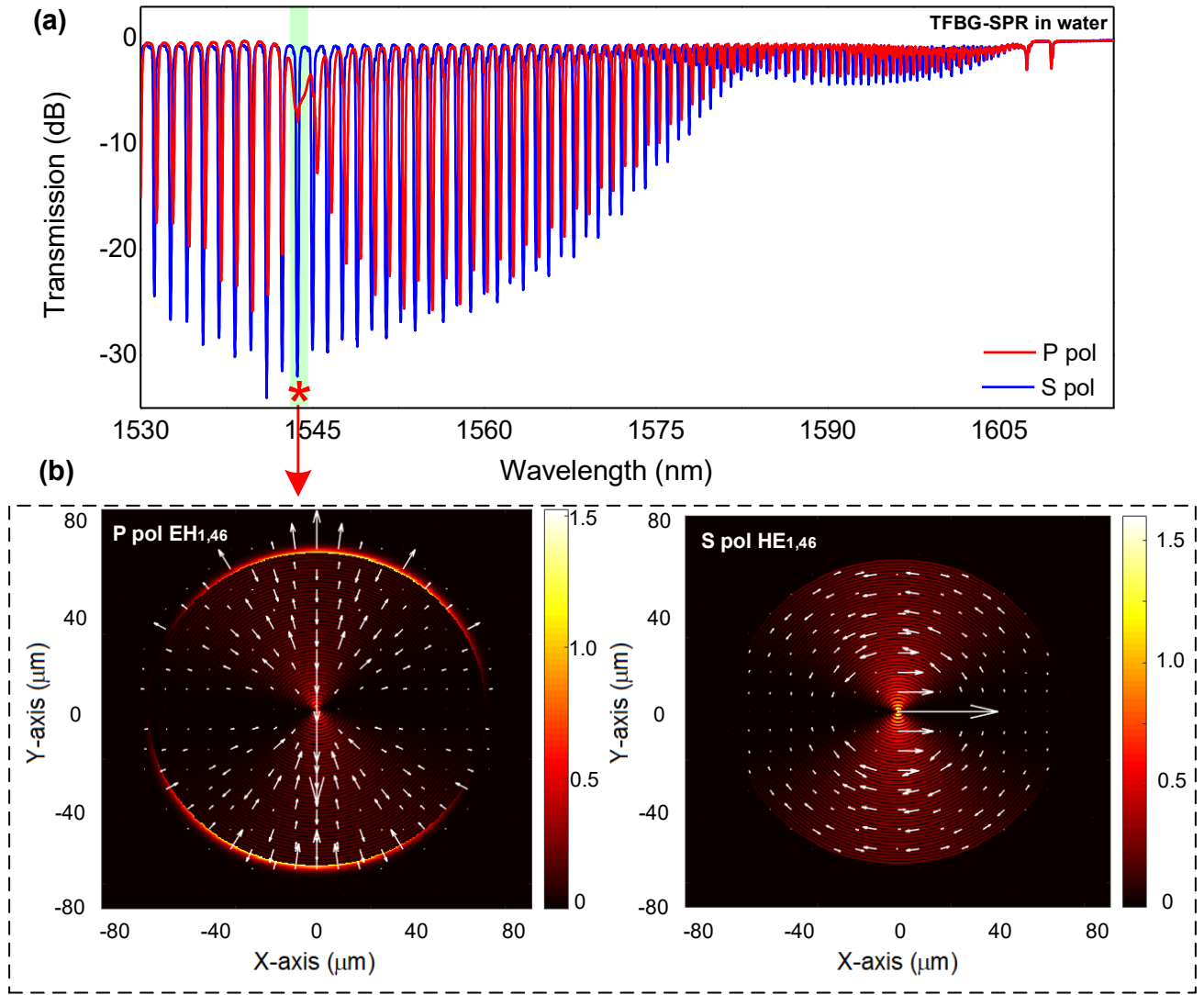

Fig. 1.2.2. Properties of TFBG-SPR: (a) Experimental polarized transmission spectra in water (red, P-pol input with SPP phase matching near $1545 \mathrm{~nm}$, and S-pol input without surface plasmon); (b) Radial (P-pol) and azimuthal (S-pol) dependence of simulated E-field intensity of plasmonic guided mode (the arrows show the E-field vector orientations in both cases).

The simulation was done by using FIMMWAVE with a $8.2 \mu \mathrm{m}$ core diameter and a refractive index of 1.449311 , a $125 \mu \mathrm{m}$ cladding diameter and a refractive index of 1.444078 , a $45 \mathrm{~nm}$ thick $\mathrm{Au}$ coating with refractive index of $0.5240+10.7427 \mathrm{i}$, and a 20 
$\mu \mathrm{m}$ layer of water above the gold (water refractive index $=1.3154$ ). As for the mode field on the left of Fig. 1.2.2 (b), the SPP is excited with an effective mode index $\mathrm{n}=1.3287+0.00129 \mathrm{i}$ and the energy density at the interface between $\mathrm{Au}$ and water is definitely enhanced. The mode power fraction in water increases from $0.68 \%$ to $25.7 \%$ and in the metal layer from $0.035 \%$ to $0.736 \%$, compared to the S-pol guided mode without SPP excitation but at a very similar wavelength (and real part of the effective index) shonw on the right of Fig. 1.2.2 (b). Because of the large dispersion of SPP modes, a small permittivity changes near the metal surface can lead to large changes in the transmission at SPP cladding mode wavelengths, hereafter referred to as surface plasmon resonance (SPR resonance). Fig. 1.2.3 shows a highly linear response of the SPR (i.e. absolute value of real part permittivity of metal is much larger than that of dielectric around $1550 \mathrm{~nm}$ wavelength) over a range of SRI from 1.33 to 1.41 , where the guided cladding modes become too lossy to build strong transmission resonances along the grating.

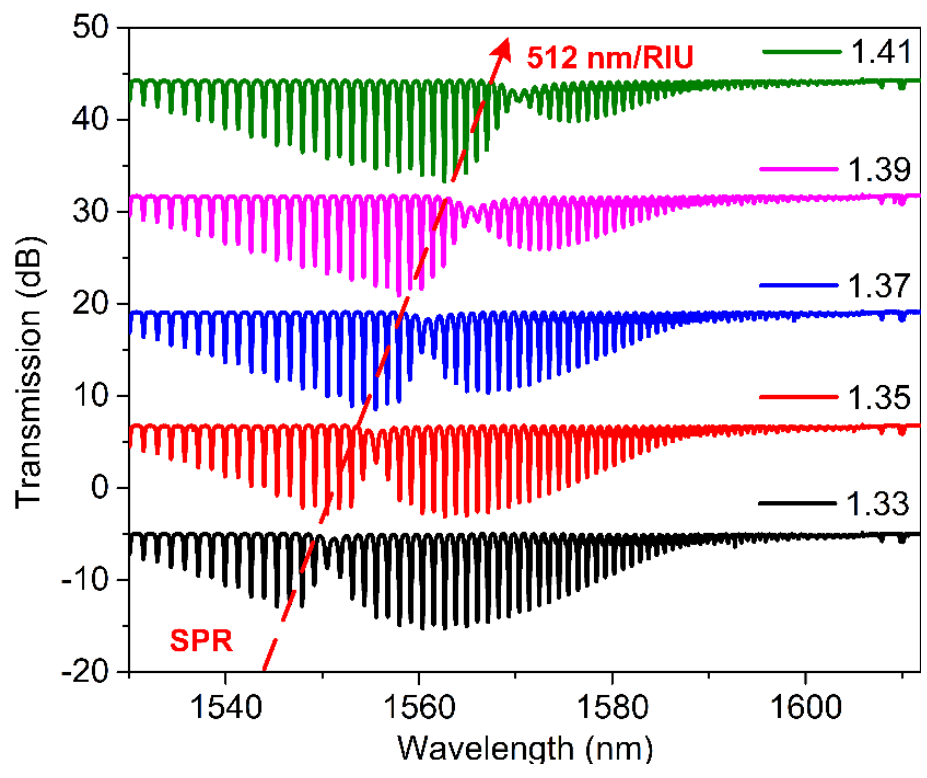

Fig. 1.2.3. Experimental spectra of TFBG-SPR as a function of the refractive index of external dielectric layer. (spectra offset to clarify the linearity of the SPR shift response with external index). 
The corresponding RI sensitivity of the SPR is around $512 \mathrm{~nm} / \mathrm{RIU}$ which is similar to that of the cut-off mode of bare fiber TFBGs, but potentially more accurate as it can be determined more accurately by following the amplitudes of a group of resonances as shown in the development of high-performance sensors in multiple areas such as biomedicine [39,56-57], electrochemical and magnetic detection [58,59], gas monitoring [60,61], and renewable energy [62].
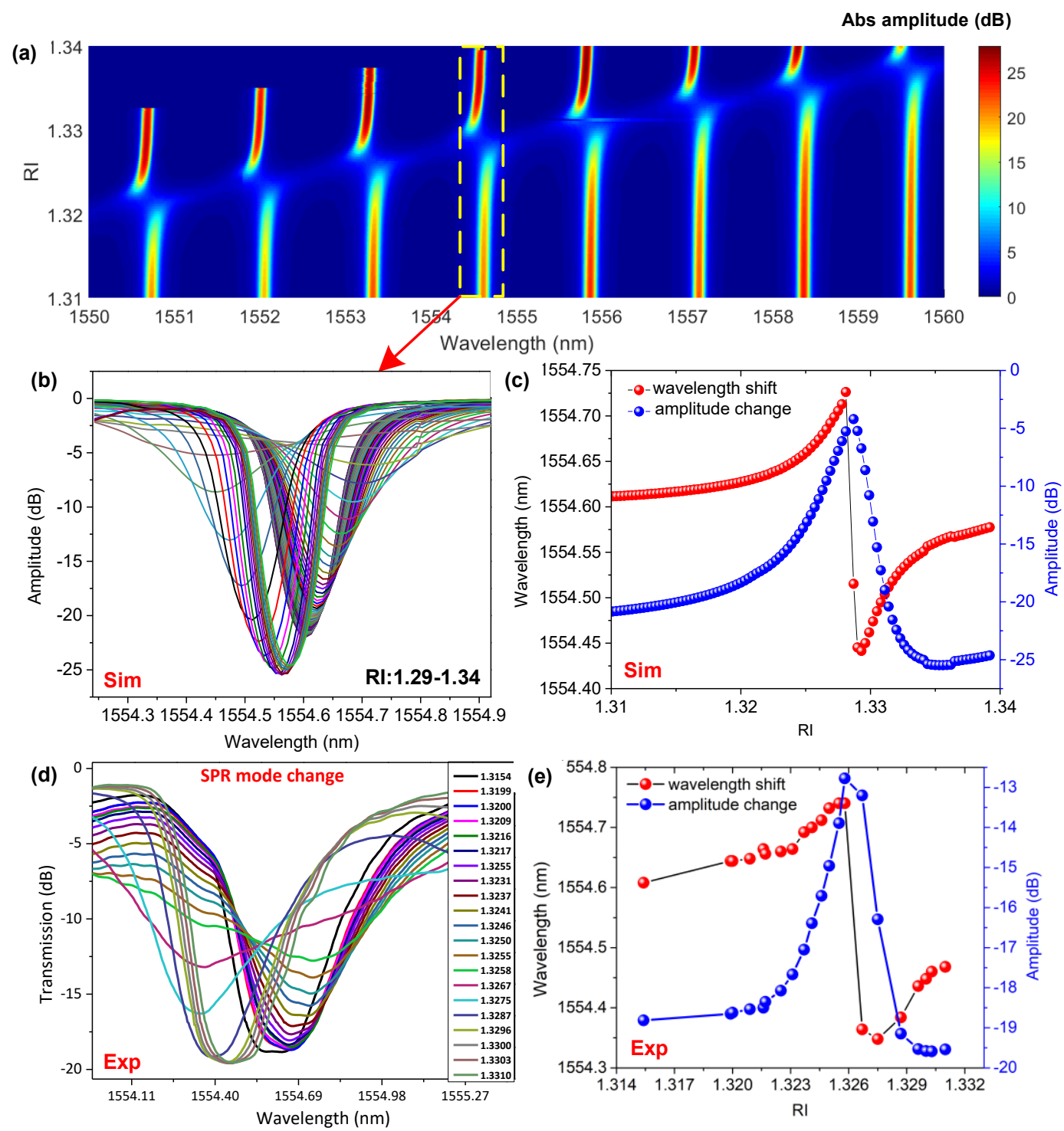

Fig. 1.2.4. Isolated hybridized cladding mode resonances near the SPR as the SRI changes (a), and detailed evolution of one resonance (a,b,c: simulations; d,e: experiment). 
The behavior of SPR in metal-coated fibers is also well understood and modelled theoretically. First, Fig. 1.2.4(a) shows the results of 300 spectrum simulations at SRI increasing gradually from 1.31 to 1.4 at 0.0003 intervals. In this case, the Au thickness was $45 \mathrm{~nm}$ and its complex refractive index was determined as a function of wavelength from the best literature values availableby the FIMMWAVE software. The TFBG had a length of $2 \mathrm{~cm}$, a core radius $=4.1 \mathrm{um}$, cladding radius $=62.5 \mathrm{um}$, cladding material of pure silica $\left(\mathrm{SiO}_{2}\right)$, and core material of germanium-doped silica with 0.0625 germanium/silicon concentration ratio (the refractive index of the silica and germanium-doped silica as a function of wavelength were also determined within FIMMWAVE). The cladding mode resonances "disappear" one after the other when the SRI increases gradually as the real part of the effective index of each mode becomes equal to the SPP effective index. The wavelength of the SPR response where the mode amplitude disappears is highly linear with SRI, confirming the observation already made in the experimental result of Fig. 1.2.3. However, the wavelength shift of the single cladding mode resonance is anomalous when the SPP passes through. Looking at cladding mode around 1554.5 nm shown in Fig. 1.2.4 (b) and (c), the resonance is shifting to longer wavelengths and accelerates to the fastest shift when the SPP is well matched and where the amplitude of the resonance attenuates the most (i.e. "disappearing" lossy mode), after which there is sudden blue shift of wavelength (wavelength "jump") and a decelerating red shift together with the amplitude recovery. All these predictions are further experimentally demonstrated in Fig. 1.2.4 (d) and (e). The reason behind this anomalous wavelength shift can be associated with a Lorentzian type relation between the real and imaginary parts of the SPP excited as in the case of SPR at visible wavelengths using a prism platform [63]. Additionally, there is an 
upper limit of RI sensitivity in terms of the rate of wavelength shift of a plasmonic hybrid cladding mode resonance (it cannot be larger than $512 \mathrm{~nm} / \mathrm{RIU}$ ) determined by the period of the grating, as discussed in [64]. The accurate calibration of the RI sensitivity of a single plasmonic hybrid cladding mode is really helpful for us to understand the sensing principle when very small permittivity perturbations are monitored such as biosensing [39,56-57] or pump-probe grating switching systems [42]. The disappearance of the resonance from the transmission spectrum, due to the strong mode loss, results in weak coupling between core mode when the input wavelength is phase matched to the "best" hybridized SPP cladding mode. Therefore, this is not an ideal choice for efficient sensing and switching. Therefore, the "weakly-matched" hybridized mode resonance immediately adjacent (on the shorter wavelength) side of the SPR total attenuation resonance is often chosen since it remains very sensitive to the SPP dispersion while keeping the imaginary part of its effective index low enough to keep a narrow linewidth (leading to more accurate wavelength and amplitude shift determinations). Fig. 1.2.5 (a) shows the average energy density profile of this adjacent resonance near the metal surface, which is 1000 times smaller than the corresponding value of the perfectly phase matched plasmon guided mode [42, Supplementary information, and Chapter 4 of this thesis]. The skin depth of the mode, where the energy density falls to $1 / \mathrm{e}$ of its maximum at the Au surface, is around $720 \mathrm{~nm}$. This is the thickness over which the TFBG can be used for sensing such as for monitoring biomedical samples attached on the surface of the fiber [39,56-57]. 
(a)

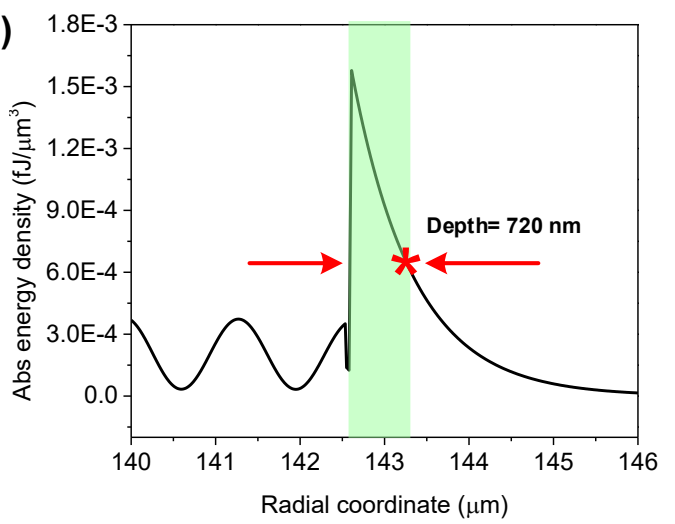

$\overline{(} \mathbf{b})$

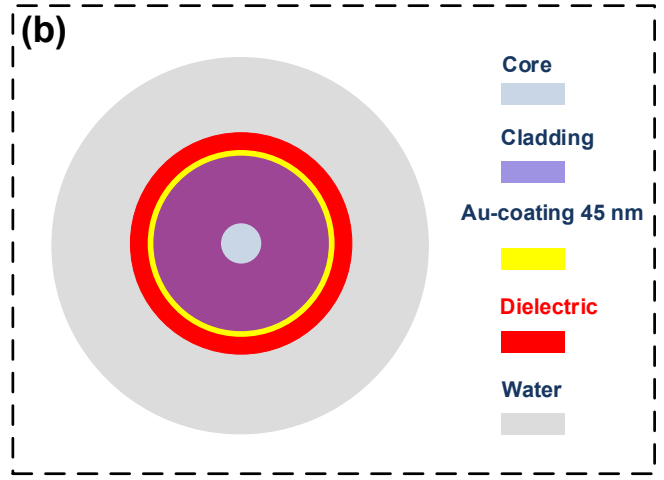

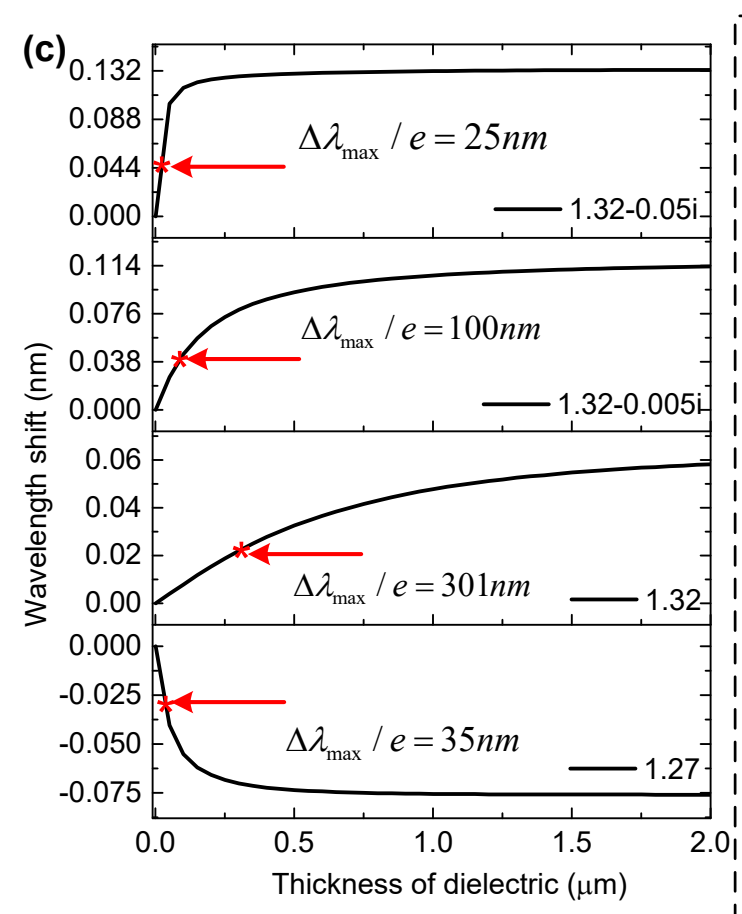

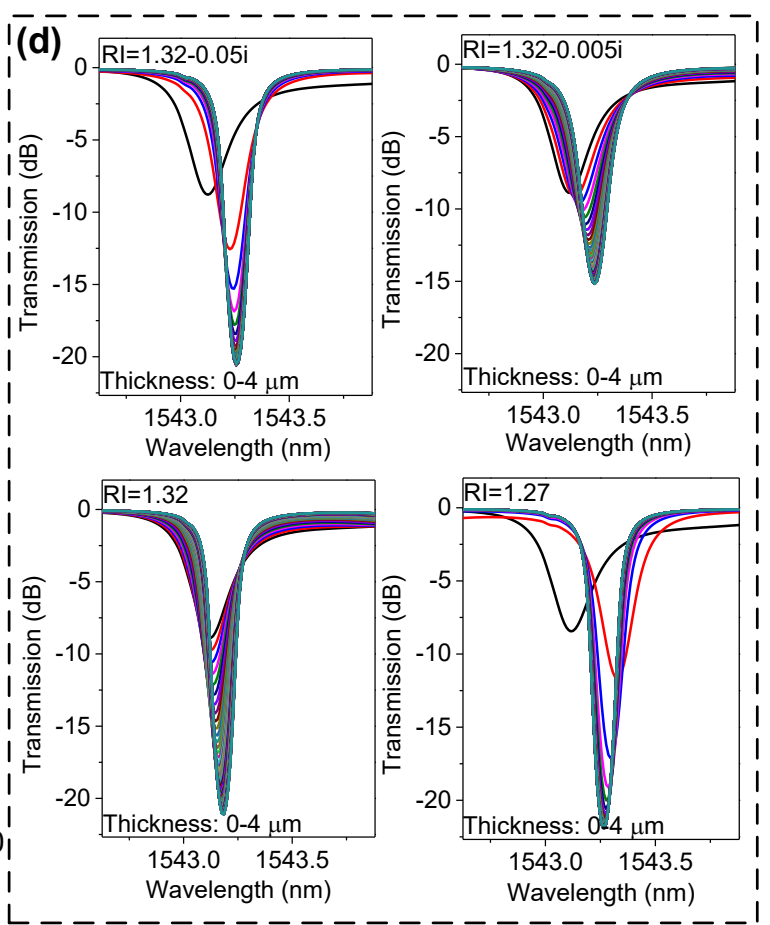

Fig. 1.2.5. (a) Radial distribution of the electric field energy density around the Au layer on the fiber cladding surface for the first adjacent resonance away from the SPR maximum (without the additional dielectric layer); (b) schematic of the Au-coated fiber with an optional additional dielectric "sensing" layer; (c) wavelength shift as a function of the thickness of dielectric layer, for different values of the complex refractive index of the additional layer (indicated by the labels on the plots). The arrows point to the thickness where the wavelength shift reaches $1 / \mathrm{e}$ of its maximum value in each case; (d) resonance transmission spectra corresponding to the situations plotted in (c). 
When adding a layer of material on the top of Au film as shown in Fig. 1.2.5 (b) (for instance when sensing for thickness changes in process monitoring or biochemical sensing), the wavelength and amplitude of this weakly matched SPR change. These changes are simulated in terms of the increasing thickness of the dielectric layer, and for several values of the complex index in Fig. 1.2.5 (c) and (d). In all cases, the wavelength shifts increase with material thickness, up to a maximum value (which is reached when the penetration depth of the mode energy is totally contained in the added thickness, so that the mode field no longer "sees" the outer layer of water). The comparative values of the thickness range for which significant wavelength shifts occur is indicated in Fig. 1.2.5 (c) by the thickness where the wavelength shifts by $1 / \mathrm{e}$ of its maximum value. This thickness decreases with increasing difference between the complex index of the added layer and that of water. It is also clear from these plots (and somewhat obvious from a theoretical standpoint) that the maximum wavelength shift increases with increasing difference in index. The fact that quite large wavelength shifts occur even if the real part of the index of the added layer remains constant but the loss increases is quite helpful to point out another great feature of TFBG-assisted SPR sensing, such as biosensing where induced losses occur when using assays involving slightly absorbing materials (such as cells and proteins) or metal nanoparticles on the surface fiber. Finally, the direction of the wavelength shift also indicates whether the real part of the index of the added layer is larger or smaller than that of the SRI.

A final simulation result is shown in Fig. 1.2.6 where the SPR region of the transmission spectrum of a Au-coated TFBG is plotted as a function of the thickness of the Au layer for thicknesses from $10 \mathrm{~nm}$ to $70 \mathrm{~nm}$. The "best" SPR response (spectrally 
narrowest and with the highest localization of cladding mode energy in the metal layer) is obtained for a thickness of $45 \mathrm{~nm}$. The broad bandwidth of SPR profile involving more than 10 cladding modes (at a thickness of $10 \mathrm{~nm}$ ) is narrowed to only one cladding mode (at a thickness around $45 \mathrm{~nm}$ ). Spectra with S-polarized input light do not show SPR, at any thickness as the cladding mode fields are TE-like at the metal interface and cannot excite SPP waves (the S-polarized spectra are very similar to bare fiber spectra apart from slightly lower resonance amplitudes and some wavelength shifts due to the gold coating; even at $10 \mathrm{~nm}$ thicknesses the Au acts as a slightly lossy mirror). This has also been demonstrated from experiment [64] (also it agrees with the result of conventional prismbased SPR linewidths as function of metal thickness [65]). The optimized thickness of metal coatings for plasmon excitation, determined from similar simulations, will be used in all sensing or switching systems presented in this thesis.
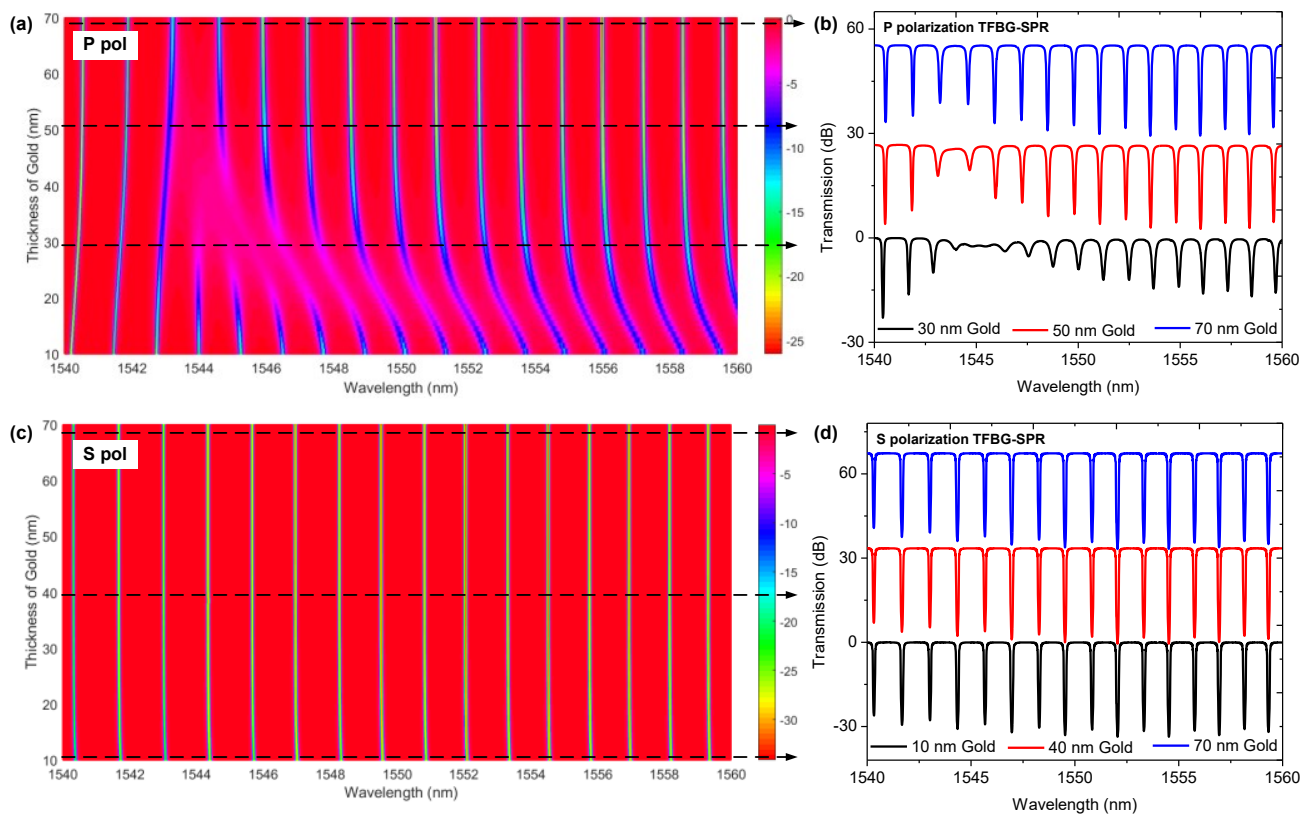

Fig. 1.2.6. Simulated TFBG-SPR Au thickness dependence: (a) P-polarized input core mode; (b) corresponding spectra with Au thickness at $30 \mathrm{~nm}, 50 \mathrm{~nm}$ and $70 \mathrm{~nm}$ of (a); (c) S-polarized input core mode; (d) corresponding spectra with Au thickness at $10 \mathrm{~nm}, 40 \mathrm{~nm}$ and $70 \mathrm{~nm}$. 


\subsubsection{Nanophotonic phenomenon based on metal particle coated-TFBG}

Localized (nanometer scale) surface plasmon resonance (LSPR) based on nanostructured metal particles strongly confines the electromagnetic of plasmon around its surface, amplifying various physical and chemical process phenomena such as surface-enhanced optical techniques [66], photovoltaics [67], and nonlinear ultrafast switching [68]. Recently, TFBGs coated with metal particles on the surface of fiber were proposed as a sensing platform. where the narrowband resonances were modulated by the permittivity perturbation of surroundings through the evanescent filed of the guided mode. For example, electromagnetic hot spots with more than two orders of magnitude enhancement were observed around randomly spaced silver nanocubes (40-100 nm edge length) coated on the surface of a TFBG at a wavelength of $647 \mathrm{~nm}$ where the LSPR is excited [69]. Also, the RI sensitivity is enhanced by coating a nanoparticle layer on surface of a TFBG, resulting in an improvement of the detection limit for avidin-biotin binding by several orders of magnitude [70], even at wavelengths in the near infrared. This points out the interesting fact that structured metal nanoparticles improve the sensing properties even far away their "natural" plasmon resonances (LSPR is mostly excited at visible wavelengths while the TFBG used are mostly for 1500-1600 nm). Therefore, combining the simple, monolithic, and low insertion loss TFBG platform with well designed nanoparticles and other light enhancing structures makes it quite promising to excite more fruitful applications which makes LSPR effects observable at telecom wavelengths.

\subsubsection{Nanophotonic phenomenon based on spherical metal particles}

Considering the LSPR for an Ag sphere immersed in water, the collective oscillation of the conduction electrons typically occurs in the visible to near-ultraviolet region of the 
spectrum [71]. Mie theory (i.e. analytical solution to Maxwell's equation describing the scattering of an electromagnetic (EM) plane wave by a homogeneous sphere (i.e. absorbing or non-absorbing considering the EM field inside and outside of the sphere object) without a particular bound on particles size by taking the form of an infinite series of spherical multipole partial waves) was used to express the metal optical properties in terms of absorption and scattering cross sections $\sigma_{a b s}$ and $\sigma_{s c a}$, which induces the generation of heat (absorption) and changing the propagation direction (scattering), respectively and they are given by

$$
\sigma_{e x t}=\sigma_{a b s}+\sigma_{s c a}
$$

where the extinction, scattering, absorption cross section are obtained by:

$$
\begin{aligned}
& \sigma_{e x t}=\frac{2 \pi}{|\vec{k}|^{2}} \sum_{L=1}^{\infty}(2 L+1) \operatorname{Re}\left\{a_{L}+b_{L}\right\} \\
& \sigma_{s c a}=\frac{2 \pi}{|\vec{k}|^{2}} \sum_{L=1}^{\infty}(2 L+1)\left(\left|a_{L}\right|^{2}+\left|b_{L}\right|^{2}\right) \\
& \sigma_{a b s}=\sigma_{e x t}-\sigma_{s c a}
\end{aligned}
$$

In which

$$
\begin{aligned}
& a_{L}=\frac{m \psi_{L}(m x) \psi_{L}^{\prime}(x)-\psi_{L}^{\prime}(m x) \psi_{L}(x)}{m \psi_{L}(m x) \eta_{L}(x)-\psi_{L}^{\prime}(m x) \eta_{L}(x)} \\
& b_{L}=\frac{\psi_{L}(m x) \psi_{L}^{\prime}(x)-m \psi_{L}^{\prime}(m x) \psi_{L}(x)}{\psi_{L}(m x) \eta_{L}(x)-m \psi_{L}^{\prime}(m x) \eta_{L}(x)}
\end{aligned}
$$

Where $m=n / n_{m}, n$ is the complex index of the metal particle and $n_{m}$ is the index of the water (both $n$ and $n_{m}$ must be set considering the wavelength dispersion), $x=|\vec{k}| R$ is he size parameter, $\psi_{L}(x)$ and $\eta_{L}(x)$ are Riccati-Bessel cylindrical functions, the $L$ is the 
order of spherical multipole excitations in the clusters ( $\mathrm{L}=1$, dipole fields; $\mathrm{L}=2$, quadrupole fields ; $\mathrm{L}=3$, octupole fields).

As for the wavelength dispersion of the permittivity of the metal particle, it should be correlated with its polycrystalline structure based on the relative contributions of bulk damping (electron-electron scattering, electron-phonon scattering, impurities and lattice defects), electron-surface scattering, radiation damping (energy loss via coupling to the radiation field) and with the different dimensions (shape, size) of the particle. As for Ag spherical nanoparticles, the dielectric function is based on bulk optical functions [72] and computed with:

$$
\varepsilon(\omega, R)=\varepsilon_{\text {Bulk }}(\omega)+\omega_{p}^{2}\left(\frac{1}{\omega^{2}+\Gamma_{\infty}^{2}}-\frac{1}{\omega^{2}+\Gamma(R)^{2}}\right)+i \frac{\omega_{p}^{2}}{\omega}\left(\frac{\Gamma(R)}{\omega^{2}+\Gamma(R)^{2}}-\frac{\Gamma_{\infty}}{\omega^{2}+\Gamma_{\infty}^{2}}\right)
$$

Where $\varepsilon_{\text {Bulk }}(\omega)$ is the bulk film permittivity of the metal including both interband and intraband contribution, which are well expressed by using a Drude model with two critical points (DCP), as discussed in Chapter 4. The additional damping parameters of the permittivity (which defines the total electron relaxation rate) and can be represented as:

$$
\Gamma(R)=\Gamma_{\infty}+\Gamma_{r}+\Gamma_{s}
$$

Where $\Gamma_{\infty}=\sum \Gamma_{i}=v_{F} / l_{\infty}\left(v_{F}=1.448 \times 10^{6} \mathrm{~m} / \mathrm{s}\right.$ is the Fermi velocity of Ag, $l_{\infty}=53.3$ $\mathrm{nm}$ is the electron mean free path of $\mathrm{Ag}[73])$.

The electron-surface scattering will play an important role when particle size becomes comparable or smaller than $l_{\infty}$, which can be calculated from a semiclassical relation:

$$
\Gamma_{s}=A \frac{v_{F} S}{4 V}
$$


Where S and V are the surface area and the volume of the average particle, respectively. $\mathrm{A}=0.1$ is a phenomenological factor.

As the particle size increase, coupling of the LSPR oscillation to the radiation field can become an important energy loss mechanism, known as radiation damping:

$$
\Gamma_{r}=2 \hbar \kappa V
$$

Where $\kappa$ is a constant that describes the magnitude of radiation damping.
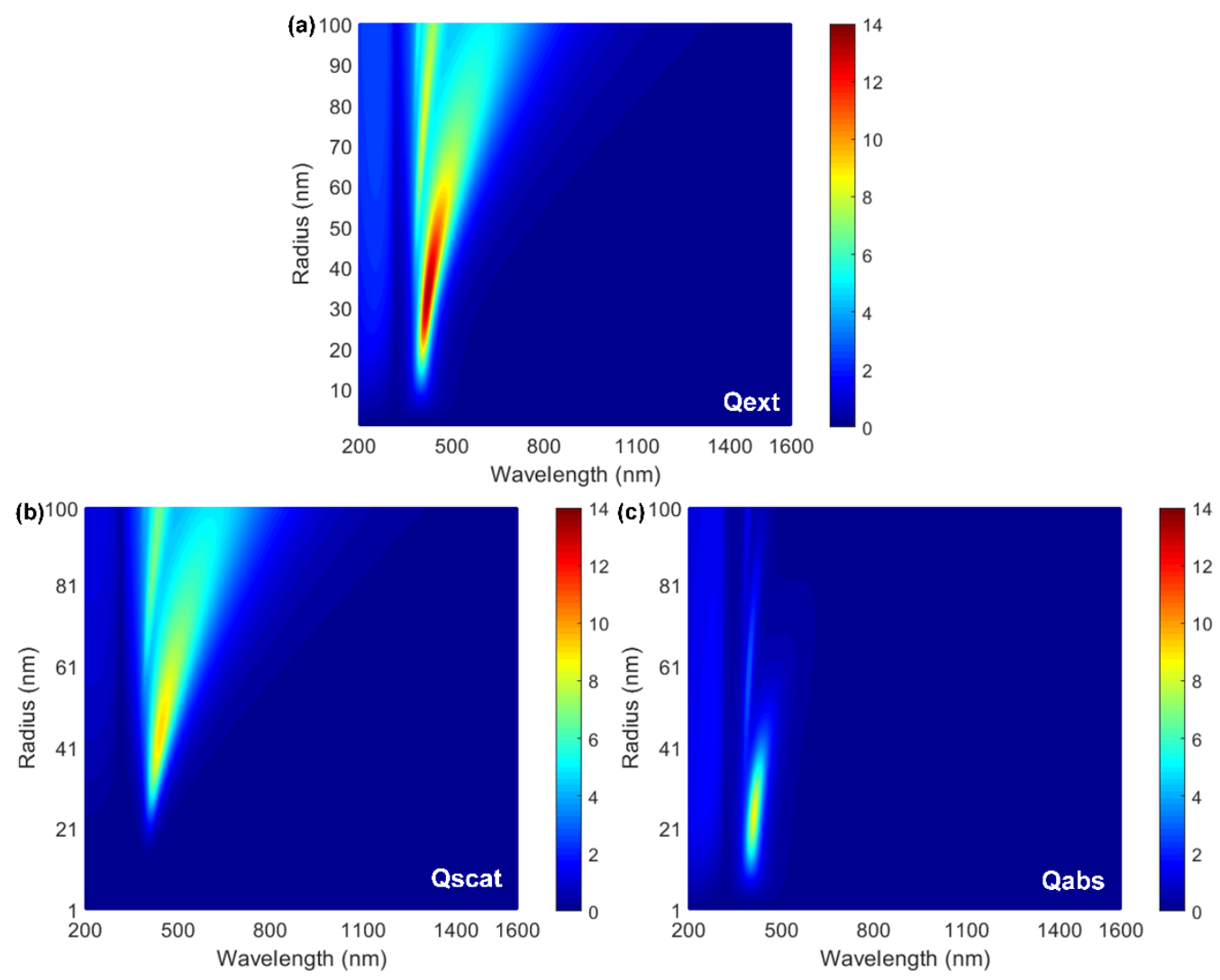

Fig. 1.2.7. The simulated extinction (a), scattering (b) and absorption (c) of Ag particles as function of radius in water.

Based on the Mie theory above, the corresponding simulated results based on for Ag particles (radius from $1 \mathrm{~nm}$ to $100 \mathrm{~nm}$ ) in water are shown in Fig. 1.2.7. The most noticeable effect when the size of the particle increases is that the plasmon resonance redshifts and broadens. The red-shift is a retardation effect, it occurs because the electric field 
is not uniform across the particle. The broadening comes from damping of LSPR in which for small particles the line width is broadened by electron-surface scattering, and for large particles radiation damping causes broadening. Moreover, when the size of particle increases, both the scattering and absorption are rising, and the absorption is larger until the radius is $32 \mathrm{~nm}$ (absorption is 2.69 (arb. units) at $399 \mathrm{~nm}$ wavelength) after which the scattering will be dominant. As for the scattering, the peak value is 9.09 (arb. units) where the radius is $45.5 \mathrm{~nm}$ at $445 \mathrm{~nm}$ wavelength. As for the absorption, the peak value is 8.367 (arb. units) where the radius is $24.5 \mathrm{~nm}$ at $412 \mathrm{~nm}$ wavelength.

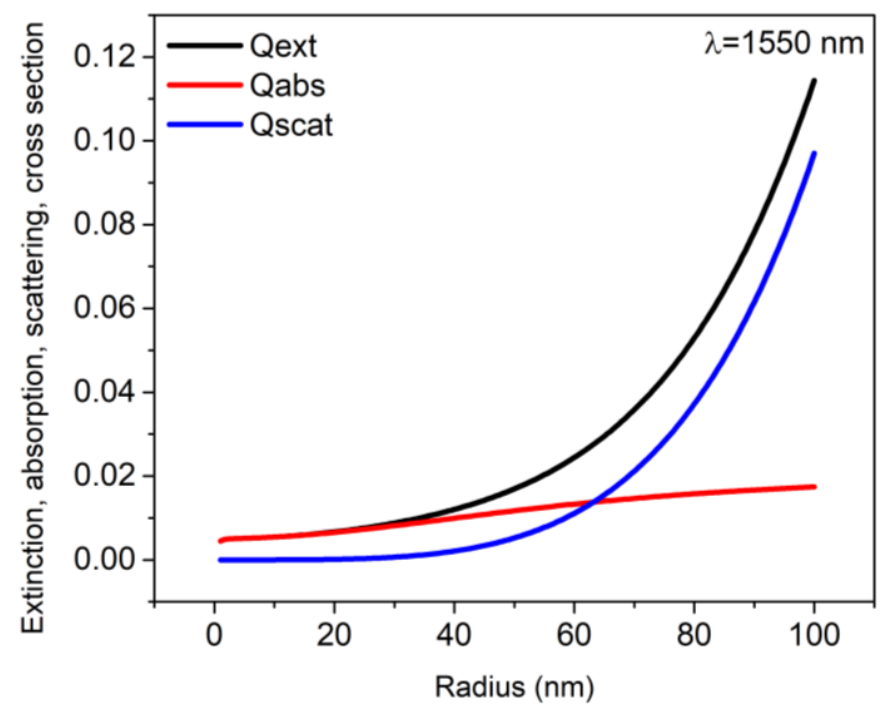

Fig. 1.2.8. The simulated extinction (a), scattering (b) and absorption (c) of Ag particles at 1550 nm wavelength.

Fig. 1.2.8 shows the extinction, scattering and absorption of a Ag particle at $1550 \mathrm{~nm}$ wavelength which is far away from the LSPR in the visible wavelength region. The scattering at a radius of $45.5 \mathrm{~nm}$ is 2561 times smaller than that around the resonance of the particle at $445 \mathrm{~nm}$ wavelength, and the absorption at a radius of $24.5 \mathrm{~nm}$ is 1114 times smaller than that at $412 \mathrm{~nm}$ wavelength. However, the TFBG sensing platform still can benefit from the enhancement coming from the metal particles working far away from their 
resonance because of the combination of the high effective surface area of metal particles together with the high $\mathrm{Q}$ factor of the cladding mode resonances. For instance, the minimum detected concentration of bio-samples improved from $90 \mathrm{nM}$ to $11 \mathrm{pM}$ and $8 \mathrm{pM}$ respectively by coating with gold nanocages ( $30 \mathrm{~nm}$ edge length, maximum absorption is at $620 \mathrm{~nm}$ ) or gold nanospheres (average diameter of $30 \mathrm{~nm}$, maximum absorption is at 520 $\mathrm{nm}$ ) on the surface of a TFBG using a $1538 \mathrm{~nm}$ working wavelength [70].

\subsubsection{Nanophotonic phenomenon based on metal rods}

It would be helpful for light-matter interactions if the resonance of the material could be moved to the near infrared region and match the cladding mode resonances of TFBGs in standard telecom single mode fibers. In this respect, a simple case of ellipsoidal particles can be considered because two localized SPR (a longitudinal resonance along the major axis and a transverse SPR across its smallest dimension) are excited, and the longitudinal resonance red shifts significantly when the aspect ratio of the rod increase [74]. This unique capability enables extremely large electric field enhancement around the rod, giving rise to a variety of light-matter interaction mechanisms, such as plasmon enhanced-ultrafast switching [75], enhancement of second harmonic generation [76], optical antenna effect [77], photothermal conversions [78], plasmon-induced vacuum Rabi splitting [79].

As for the linear principle of nanorod, we assume the particles are much smaller than the wavelength of the light, i.e. in the so-called "quasi-static" limit (where phase retardation and effects of higher multipoles are neglected), and only the dipole contributions are important. The extinction is then dominated by absorption. The absorption for ellipsoidal particles was developed in [80] as:

$$
\sigma_{\text {abs }}(\omega)=\frac{2 \pi}{3 \lambda} \varepsilon_{\text {water }}^{3 / 2} V \sum_{j=1-3} \frac{\left(1 / P_{j}^{2}\right) \varepsilon_{\text {imag }}(\omega)}{\left[\varepsilon_{\text {real }}(\omega)+\left(1-P_{j}\right) \varepsilon_{\text {water }} / P_{j}\right]^{2}+\varepsilon_{\text {imag }}(\omega)^{2}}
$$


Where $V$ is the particle volume, $\varepsilon(\omega)$ is the metal particle dielectric function, and the depolarization factors $P_{j}$ for three axes: A, B, C of the rod with $A>B=C$ are:

$$
\begin{gathered}
P_{A}=\left(\frac{1-e^{2}}{e^{2}}\right)\left\{\frac{1}{2 e} \ln \left(\frac{1+e}{1-e}\right)-1\right\} \\
P_{B}=P_{C}=\frac{1-P_{A}}{2} \\
e=\sqrt{1-(B / A)^{2}}
\end{gathered}
$$

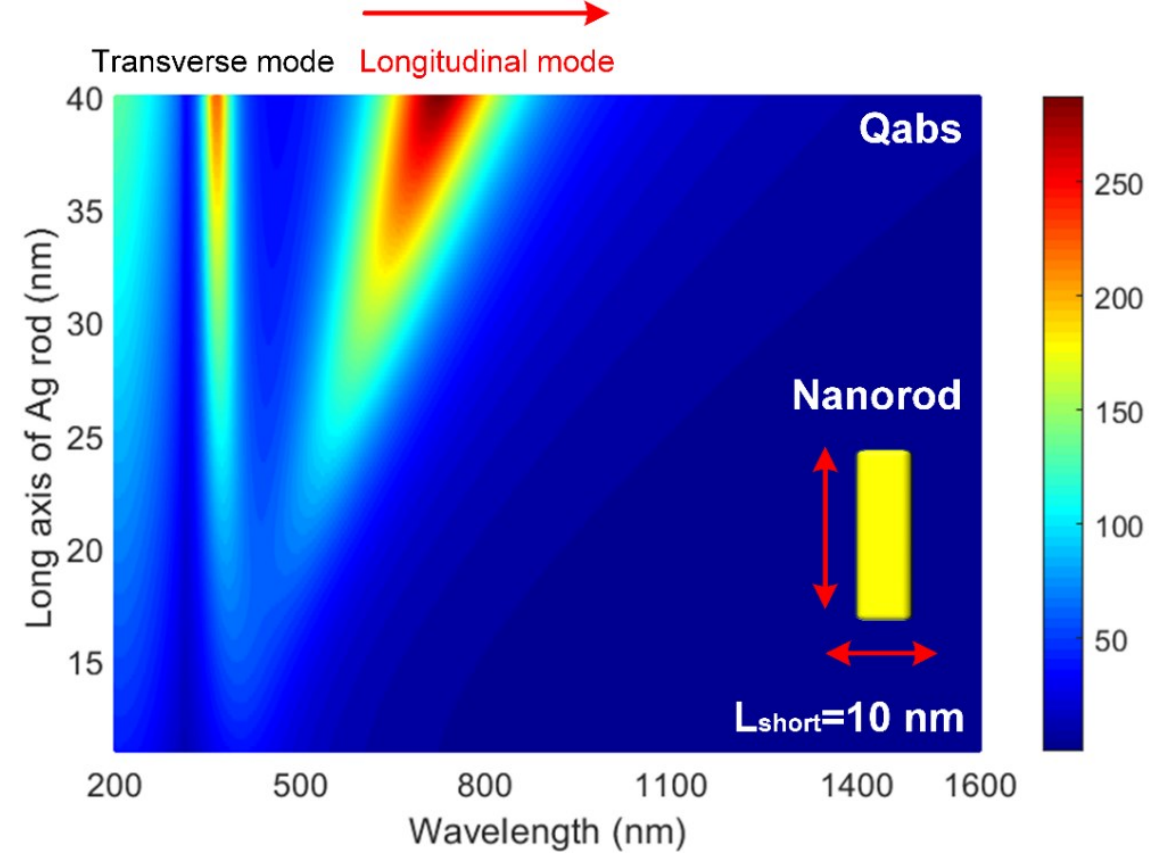

Fig. 1.2.9. The simulated absorption of an $\mathrm{Ag}$ nanorod as a function of long axis length (the short axis was fixed at $10 \mathrm{~nm}$ ) in water.

Fig. 1.2.9 shows the simulated absorption spectra corresponding to transverse and longitudinal resonance for a $\mathrm{Ag}$ nanorod $(10 \mathrm{~nm}$ for the short axis and the long axis changing from $10 \mathrm{~nm}$ to $40 \mathrm{~nm}$ ). The absorption maximum of the transverse mode shifts to shorter wavelength with increasing aspect ratio, which agrees with the results of Martin and coworkers [81] obtained with randomly orientated nanorods as predicted by Gan's 
theory. The maximum absorption of the longitudinal mode red-shifts with increasing Ag nanorod aspect ratio, showing a variation of over $100 \mathrm{~nm}$. Furthermore, the relative intensity ratio of the longitudinal to the transverse mode increases. Therefore, it is quite promising to move the longitudinal mode towards $1550 \mathrm{~nm}$, such as the specially designed nanorod or a pair of nanorods with strong coupling (Fig. 1.2.10) [82], or the high dielectric constant material coated nanorod (Fig.1.2.11) [83].
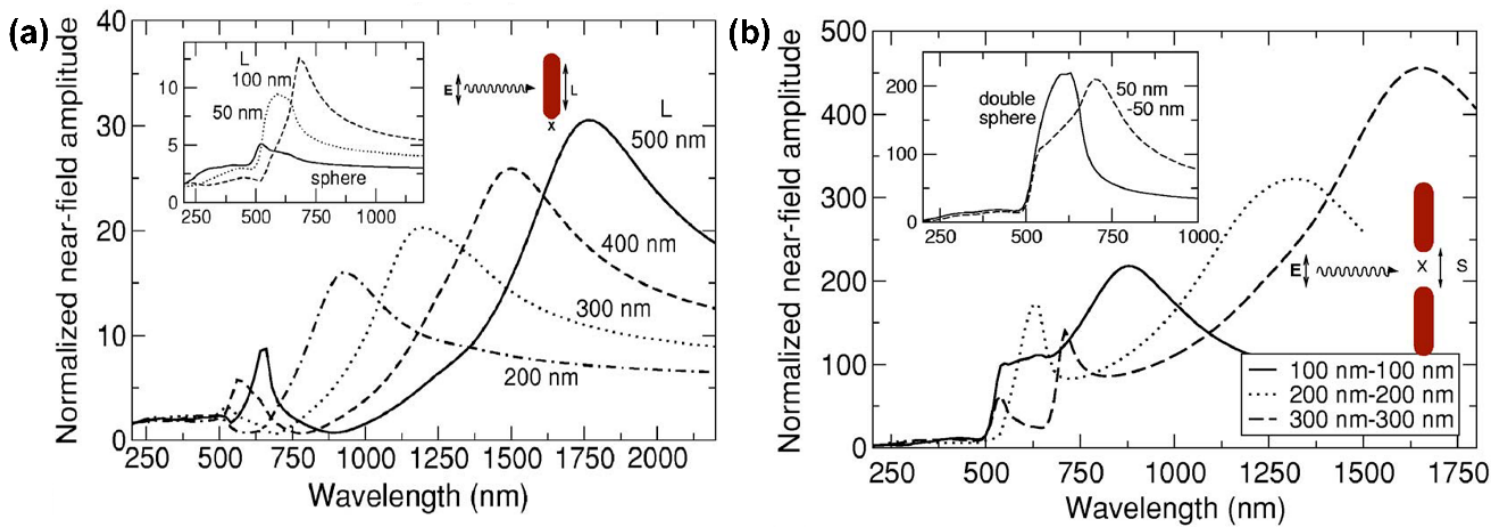

Fig. 1.2.10. Near-field intensity of LSPR for (a) cylindrical Au nanorod with radius R of $40 \mathrm{~nm}$; (b) a pair of identical cylindrical Au nanorods with $2 \mathrm{~nm}$ gap and $\mathrm{R}=40 \mathrm{~nm}$ (The inset shows the results of two coupled sphere) (reproduced from [82]).
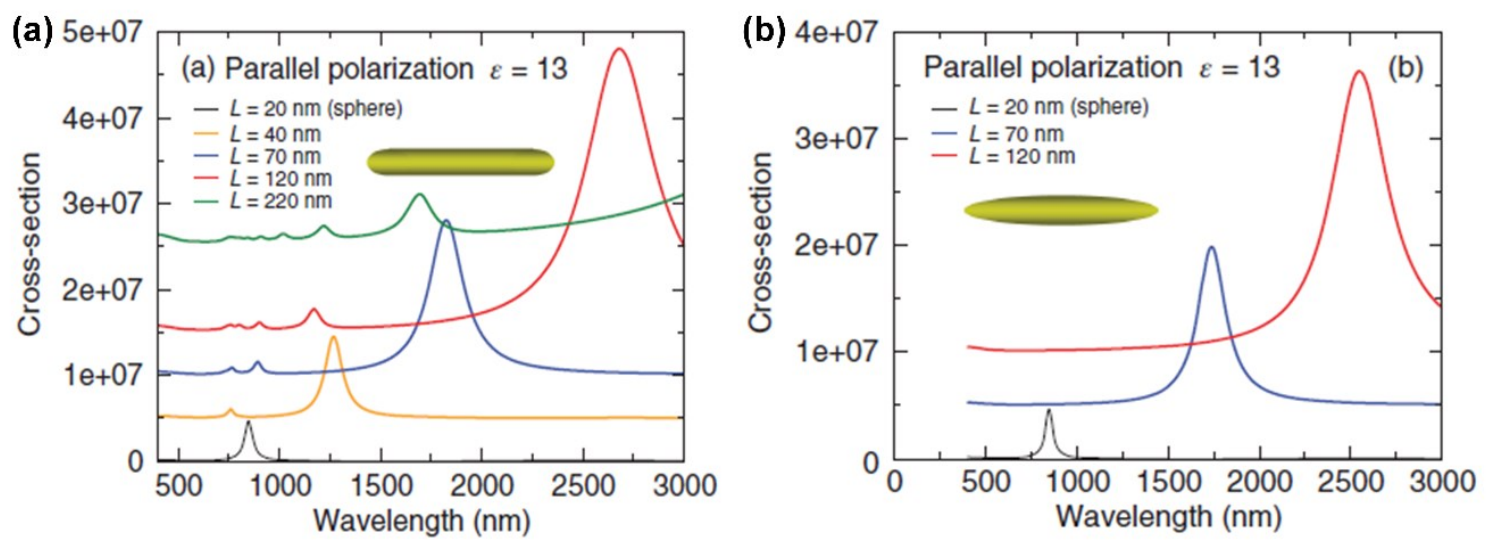

Fig. 1.2.11. Scattering cross section for gold nanoparticles embedded in a medium with a large dielectric constant: (a) nanorods, (b) ellipsoidal nanoparticles. The nanoparticle radius is $\mathrm{R}=10 \mathrm{~nm}$, excited by a plane wave polarized along their long axis (reproduced from [83]). 
As for $\mathrm{Ag}$ nanowires used at $1550 \mathrm{~nm}$ wavelength [84,85], J. M. Renoirt and coworkers [85] proposed an excellent work in terms of surface plasmon resonance in oriented Ag nanowires deposited on TFBGs. The nanowires with 1-3 $\mu \mathrm{m}$ in length and diameters of $0.04-0.05 \mu \mathrm{m}$ and $40 \%$ surface coverage shown in Fig. 1.2.12 (a) were first measured dispersed in solution and they show a small absorption peak near $500 \mathrm{~nm}$ corresponding to the short axis of the wires, and a broad absorption tail extending across the near infrared range (there is no peak of absorption in the long wavelength range, which means that a specific LSPR is not excited, but these nanowires were randomly oriented). However, when the nanowires were partially aligned in the aziumuthal direction on the TFBG surface, they behaved very much as a continuous metal thin film with a well-defined SPR response (as evidenced by the PDL difference between a TFBG with oriented and non oriented nanowires shown in Fig. 1.2.12 (c),(d)).
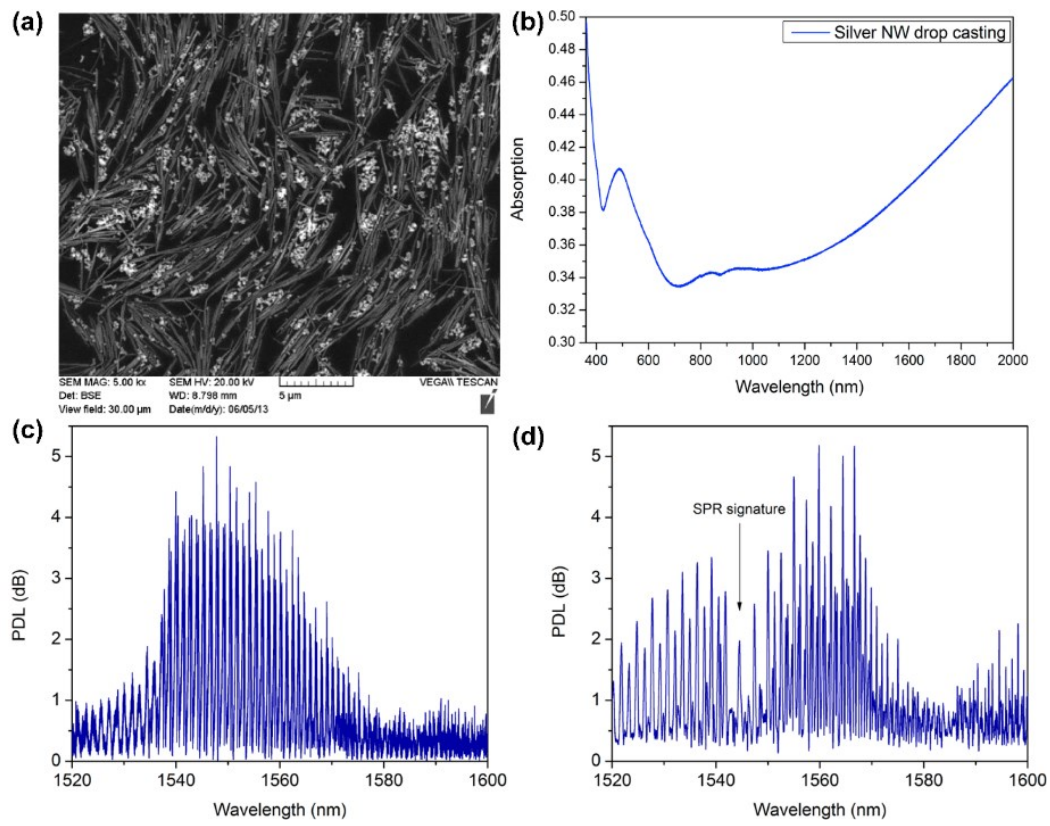

Fig. 1.2.12. (a) SEM image of aligned nanowires perpendicular to the fiber axis; (b) the UV-visNIR spectrum of the nanowires solution; (c) PDL spectrum of a TFBG coated with randomly oriented nanowires; (d) and with orthogonally aligned nanowires (reproduced from [85]). 


\subsubsection{Nanophotonic phenomena based on metal films at percolation}

Percolation in metal films is defined as the point (during deposition or etching for instance) where an insulator-metal phase transition occurs. At percolation, the "metal" film shows remarkable optical properties resulting from localization of plasmons leading to extremely strong confinement of electromagnetics filed in sharp nm-sized volumes called "hot spots." The local field enhancements associated with the hot spots amplify the nonlinear optical processes such as Kerr optical nonlinearities [86,87], surface enhanced Raman scattering [88], and enhancements of second harmonic generation [89-91]. Metal layers at percolation can be produced by thermal evaporation or sputtering of metal onto an insulatiing substate where the metal filling factor is gradually increased [92,93], and the coalescence between initially isolated metal granules results in the formation of irregularly shaped fractal clusters of metal particles. As we have seen, it is possible to detect the optical properties of thin metal films on the surface of TFBGs and thus to detect the percolation threshold. In this respect the polarization control of the probing evanescent field (i.e. either parallel or perpendicular to the thin film, by using S- or P-polarized input light) by the TFBG is critical since percolation (i.e. sudden changes in conductivity) is only meaningful for electron motion in the plane of the film. Preliminary work in the measurements of thin film optical properties by tracking resonance amplitude and wavelength shifts with TFBGs at wavelengths between 1500 and $1600 \mathrm{~nm}$ was carried out in the following cases: Chemical vapour deposition (CVD) of $\mathrm{Au}$ and $\mathrm{Cu}$ [94-96], electron-beam evaporation of $\mathrm{Au}$ [97], electroless plating of $\mathrm{Au}$ [98], and atomic layer deposition (ALD) of $\mathrm{Al}_{2} \mathrm{O}_{3}$ [99]. 


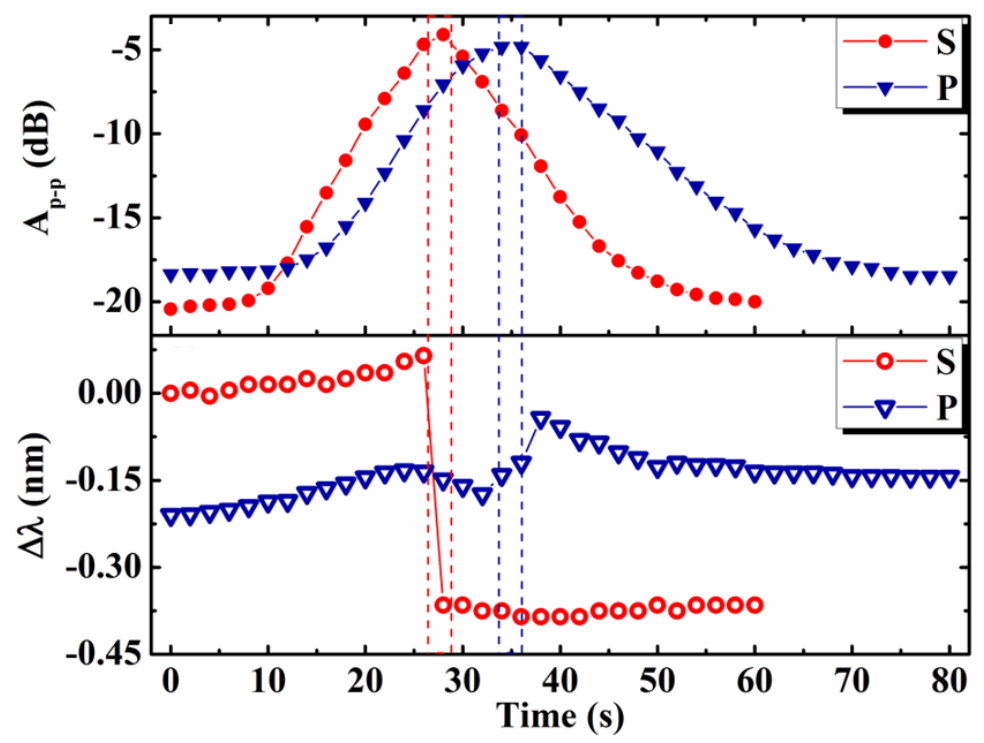

Fig. 1.2.13. Cladding mode response to gold nanoparticle grown by CVD for P- and S- polarized incident input (reproduced from [95]).

For instance, Fig. 1.2.13 shows experimental results of cladding modes resonances (1 cm in length, 10-degree TFBG) responding to the gold film growth real time during (CVD) under vacuum, as the gold film changes from isolated nanoparticles to a continuous film. Basically, both the S- and P-polarized resonances go through a similar evolution in that cladding modes become much attenuated at first, and then recover to a strong strength. Meanwhile, a very large wavelength shift (i.e. $0.45 \mathrm{~nm}$ blue shift) for S-polarized light occurs at the same time as the resonance amplitude collapses totally, while the P-polarized cladding modes shifts much less (i.e. $0.1 \mathrm{~nm}$ red shift) as shown Fig. 1.2.13. New results from Chapter 2 of this thesis on gradual etching of a Ag coating now indicate that the special signature that was observed for the S-mode during CVD Ag deposition more than 6 years ago corresponds in fact to the percolation threshold (thanks to simultaneous measurements of film conductivity during etching that show a large change in conductivity when the S-mode amplitude collapses and its wavelength suddenly blue shifts). 


\subsection{Organization of thesis}

This thesis is organized in the following manner. Chapter 1 provides an introduction to the problem, motivation, and general contents of this thesis. It also contains a literature review containing the basic optical properties of TFBG and of coated TFBGs (including functional materials, metal nanospheres, nanorods, and metal films at percolation). A list of research output published or presented during this $\mathrm{PhD}$ is also included, with descriptions of the author's contributions in each case.

Chapter 2 reproduces the research article entitled "Optical detection of the percolation threshold of nanoscale silver coatings with optical fiber gratings," by Fu Liu, Xuejun Zhang, Tuan Guo, and Jacques Albert, presently under review at APL Photonics. This article shows the in-situ, real-time measurement of the TFBG spectra at wavelengths near $1550 \mathrm{~nm}$ to monitor the metal-to-dielectric transition of silver films coated on surface of grating during wet etching. Confirmation of the percolation threshold is obtained from simultaneous measurements of the silver film conductivity, corresponding to the total collapse of all cladding modes resonance polarized azimuthally in the TFBG transmission spectrum.

Chapter 3 reproduces the research article "Saturable absorption and bistable switching of single mode fiber core-guided by a $6 \mathrm{~nm}$-thick graghene coating on the cladding surface," by F. Liu, M. Qi, T. Guo, and J. Albert, accepted for publication in Annalen der Physik. This article shows that the coupling of less than $80 \mu \mathrm{W}$ of in-plane polarized nearinfrared light in a $6 \mathrm{~nm}$-thick graphene layer deposited on TFBG produces important permittivity changes leading to bistability and self-starting $50 \%$ modulation of over $1 \mathrm{~W}$ of continuous wave light in the core. The pulse repetition rate of the modulation increases 
from 10 to $269 \mathrm{~Hz}$ for input powers ranging from 0.3 to $1.33 \mathrm{~W}$ in the core, which results from photo-induced Joule heating in the graphene layer giving rise to temperature increases of the order of $60^{\circ} \mathrm{C}$ and corresponding permittivity changes in the graphene and underlying silica fiber.

Chapter 4 reproduces the research article " $40 \mathrm{GHz}$-rate all-optical cross-modulation of core-guided near infrared light in single mode fiber by surface plasmons on gold-coated tilted fiber Bragg gratings," by F. Liu and J. Albert, APL Photon. 4, 126104 (2019). This article shows that a 25 ps-time scale switching of continuous wave light is achieved by pump pulses pumping plasmonic hybrid guided cladding modes near $1550 \mathrm{~nm}$ due to hot carriers from the SPPs in a $45 \mathrm{~nm}$-thin gold coating on the fiber cladding, leading to modification of metal complex permittivity on a time scale of the order of picoseconds and hence modulating the cladding mode resonance wavelengths and amplitude of the TFBG.

Chapter 5 shows the anti-reflection properties of a partially etched Ag film at percolation coated on surface of a fiber grating. The far-field scattering of the special film is measured at near-infrared wavelengths. This investigation will be continued as future work.

Chapter 6 summarizes the main results proposed in this thesis.

Some repetition between chapters is unavoidable, such as the fabrication of TFBGs from chapter 2 to chapter 4 , because this is an "integrated thesis" which reproduces the actually facsimile copies of published or submitted papers. The references for each chapter follow the chapter instead of being grouped at the end. In response to comments and questions raised during the oral thesis defense, minor additions (clarifications) were made to the text in Chapters 3-4, so that they are no longer exact replicas of the published papers. 
Furthermore, Chapter 2 is undergoing minor revisions in response to the review process at the journal where it was submitted, so again the published version will be slightly different.

This doctoral project resulted in the following publications:

Publications in peer-reviewed journals:

1. F. Liu, X. Zhang, T. Guo, and J. Albert*, "Optical detection of the percolation threshold of nanoscale silver coating with optical fiber gratings," APL Photonics, under review.

Contribution: F. L. carried out the experiments and analyzed the data; X. Z and T. G. fabricated silver coated sample; J. A. supervised the project and analyzed the data; F. L. and J. A. co-wrote the manuscript.

2. F. Liu, M. Qi, T. Guo, and J. Albert*, "Saturable absorption and bistable switching of single mode fiber core-guided light by a $6 \mathrm{~nm}$-thick, few layers graphene coating on the cladding surface, " Annalen der Physik, 2000157 (2020).

Contribution: F. L. carried out the experiments and analyzed the data; M. Q. and T. G. fabricated the graphene sample; J. A. supervised the project and analyzed the data; F. L. and J. A. co-wrote the manuscript.

3. F. Liu, J. Albert*, "40 GHz-rate all-optical cross-modulation of core-guided near infrared light in single mode fiber by surface plasmons on gold-coated tilted fiber Bragg gratings," APL Photonics 4, 126104 (2019).

Contribution: F. L. carried out the experiments and analyzed the data; J. A. supervised the project and analyzed the data; F. L. and J. A. co-wrote the manuscript.

4. T. Gang, F. Liu, M. Hu, and J. Albert*, "Integrated differential area method for vaviable sensitivity interrogation of tilted fiber Bragg grating sensors," J. Light. 
Technol. 37, 4531-4536 (2019).

Contribution: T. G. carried out the experiments; F. L and T. G. analyzed the data; J. A. supervised the project and analyzed the data; T. G and J. A. co-wrote the manuscript.

5. J. Albert* (supervisor), F. Liu, V. Marquez-Cruz, "Hypersensitivity and application of cladding modes of optical fibers coated with nanoscale metal layers," Sensors 18, 1518 (2018).

Contribution: J.A. designed the study, performed all the data analyses, and wrote the paper; F.L. performed all of the simulations; V.M.-C. performed all the experiments and data analysis for Section 3.4.

6. J. J. Lao (co-first author), P. Sun (co-first author), F. Liu, X. J. Zhang, Ch. X. Zhao, W. J. Mai*, T. Guo*, G. Z. Xiao, and J. Albert, "In situ plasmonic optical fiber detection of the state of charge of supercapacitors for renewable energy storage," Light Sci. Appl. 7, 34 (2018).

Contribution: T.G. and W.J.M. conceived the sensors, supervised the project, and analyzed the data; J.L. P.S. and F. L. carried out the experiments; Theoretical analyses were done by G.X. and J.A. All authors contributed to the preparation of the manuscript.

7. X. J. Zhang, S. S. Cai, F. Liu, H. Chen, P. G. Yan, Y. Yuan, T. Guo*, and J. Albert, "In situ determination of the complex permittivity of ultrathin H2-infused palladium coatings for plasmonic fiber optic sensors in the near infrared," J. Mater. Chem. C 6, $5161-5170(2018)$. 
Contribution: X. J. Z. performed the experiment; Z. J. Z, S. S. C and F. L. analyzed the data; T. G. and J. A. supervised the project, and carried out the theory simulation; All authors contributed to the preparation of the manuscript.

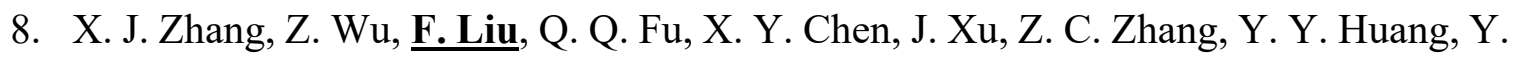
Tang, T. Guo*, J. Albert, "Hydrogen peroxide and glucose concentration measurement using optical fiber grating sensors with corrodible plasmonic nanocoatings," Biomed. Opt. Express 9 (4), 1735-1744 (2018).

Contribution: X. J. Z. performed the experiment; Z. J. Z, Z. W. and F. L. analyzed the data and performed the simulation; T. G. and J. A. supervised the project; All authors contributed to the preparation of the manuscript.

\section{Conference papers:}

1. F. Liu, and J. Albert*, "All-optical ultrafast switching based on plasmon-generated hot carriers in gold-coated fiber gratings," Laser Science to Photonic Applications (CLEO) (San Jose, USA), May. 2019. (Oral presentation)

2. F. Liu, and J. Albert*, "Anti-reflection for fiber cladding modes by silver island film coating," Photonics North (Quebec City, Canada), June. 2019. (Oral presentation)

3. T. Gang, F. Liu, M. Hu, and J. Albert*, "Tilted fiber Bragg grating interrogation method with adjustable and increased sensitivity," 26 th Optical Fiber Sensors (Lausanne, Switzerland), Sep. 2018. (Poster presentation)

\subsection{References}

1. V. Sasikala, and K. Chitra, "All optical switching and associated technologies: a review," J Opt. 47, 307-317 (2018). 
2. Y. D. $\mathrm{Wu}$, and T. T. Shih, "New all-optical logic gates based on the local nonlinear Mach-Zehnder interferometer," Opt. Express 16, 248-257 (2008).

3. C. Sturm, D. Tanese, H. S. Nguyen, H. Flayac, E. Galopin, A. Lemaitre, I. Sagnes, D. Solnyshkov, A. Amo, G. Malpuech, and J. Bloch, "All-optical phase modulation in a cavity-polariton Mach-Zehnder interferometer," Nat. Commun. 5, 3278 (2014).

4. H. Zhao, W. S. Fegadolli, J. Yu, Z. Zhang, L. Ge, A. Scherer, and L. Feng, "Metawaveguide for asymmetric interferometric light-light switching," Phys. Rev. Lett. 117, 193901 (2016).

5. M. Bradford, K. C. Obi, and J. T. Shen, "Efficient single-photon frequency conversion using Sagnac interferometer," Phys. Rev. Lett. 108, 103902 (2012).

6. A. C. Ferreira, M. B. C. Costa, A. G. Coelho, C. S. Sobrinho, J. L. S. Lima, J. W. M. Menezes, M. L. Lyra, and A. S. B. Sombra, "Analysis of the nonlinear optical switching in a Sagnac interferometer with non-instantaneous Kerr effect," Opt. Commun. 285, 1408-1417 (2012).

7. J. Wu, T. Moein, X. Xu, and D. J. Moss, "Advanced photonic filters based on cascaded Sagnac loop reflector resonators in silicon-on-insulator nanowires," APL Photonics 3, 046102 (2018).

8. S. Pricking, M. Vieweg, and H. Giessen, "Influence of the retarded response on an ultrafast nonlinear optofluidic fiber coupler," Opt. Express 19, 21673-21679 (2011).

9. R. Schiek, A. S. Solntsev, and D. N. Neshev, "Temporal dynamics of all-optical switching in quadratic nonlinear directional couplers," Appl. Phys. Lett. 100, 111117 (2012). 
10. K. Ogusu, "All-optical switching in nonlinear multimode interference couplers," Jpn. J. Appl. Phys. 51, 082503 (2012).

11. K. Ogusu, and H. Li, "Normal-mode analysis of switching dynamics in nonlinear directional couplers," J. Lightw. Technol. 31, 2639-2656 (2013).

12. V. Eckhouse, I. Cestier, G. Eisenstein, S. Combrie, G. Lehoucg, and A. D. Rossi, "Kerrinduced all-optical switching in a GaInP photonic crystal Fabry-Perot resonator," Opt. Express 20, 8524-8534 (2012).

13. L. Jiang, J. Guo, L. Wu, X. Dai, and Y. Xiang, "Manipulating the optical bistability at terahertz frequency in the Fabry-Perot cavity with graphene," Opt. Express 23, 3118131191 (2015).

14. A. A. Ryzhov, I. M. Belousova, Y. Wang, H. Qi, and J. Wang, "Optical limiting properties of a nonlinear multilayer Fabry-Perot resonator containing niobium pentoxide as nonlinear medium," Opt. Lett. 39, 4847-4850 (2014).

15. J. S. Pele, K. Rivoire, S. Vo, C. Santori, D. A. Fattal, and R. G. Beausoleil, "Picosecond all-optical switching in hydrogenated amorphous silicon microring resonators," Opt. Express 22, 3797-3810 (2014).

16. W. Yoshiki, and T. Tanable, "All-optical switching using Kerr effect in a silica toroid microcavity," Opt. Express 22, 24333-24341 (2014).

17. J. Volz, M. Scheucher, C. Junge, and A. Rauschenbeutel, "Nonlinear $\pi$ phase shift for single fibre-guided photons interacting with a single resonator-enhanced atom," Nature Photon. 8, 965-970 (2014).

18. A. Martinez, J. Blasco, P. Sanchis, J. V. Galan, J. G. Ruperez, E. Jordana, P. Gautier, Y. Lebours, S. Hernandez, R. Spano, R. Guider, N. Daldosso, B. Garrido, J. M. Fedeli, 
L. Pavesi, and J. Marti, "Ultrafast all-optical switching in a silicon-nanocrystal-based silicon slot waveguide at telecom wavelength," Nano Lett. 10, 1506-1511 (2010).

19. Y. Fu, X. Hu, C. Lu, S. Yue, H. Yang and Q. Gong, "All-optical logic gates based on nanoscale plasmonic slot waveguides," Nano Lett. 12, 5784-5790 (2012).

20. K. Nozaki, T. Tanabe, A. Shinya, S. Matsuo, T. Sato, H. Taniyama, and M. Notomi, "Sub-femtojoule all-optical switching using a photonic-crystal nanocavity," Nature Photon. 4, 477-483 (2010).

21. N. Mattiucci, M. J. Bloemer, and G. D. Aguanno, "All-optical bistability and switching near the Dirac point of a 2-D photonic crystal," Opt. Express 21, 11862-11868 (2013).

22. Z. Li, and Z. Meng, "Polystyrene Kerr nonlinear photonic crystals for building ultrafast optical switching and logic devices," J. Mater. Chem. C 2, 783-800 (2014).

23. M. R. Shcherbakov, P. P. Vabishchevich, A. S. Shorokhov, K. E. Chong, D. Y. Choi, I. Staude, A. E. Miroshnichenko, and D. N. Neshev, "Ultrafast all-optical switching with magnetic resonances in nonlinear dielectric nanostructure," Nano Lett. 15, 69856990 (2015).

24. A. V. Krasavin, T. P. Vo, W. Dickson, P. M. Bolger, and A. V. Zayats, "All-plasmonic modulation via stimulated emission of copropagating surface plasmon polaritons on a substrate with gain," Nano Lett. 11, 2231-2235 (2010).

25. G. A. Wurtz, R. Pollard, W. Hendren, G. P. Wiederrecht, D. J. Gosztola, V. A. Podolskiy, and A. V. Zayats, "Designed ultrafast optical nonlinearity in a plasmonic nanorod metamaterial enhanced by nonlocality," Nat. Nanotechnol. 6, 107-111 (2011).

26. M. Kauranen, and A. V. Zayats, "Nonlinear plasmonics," Nature Photon. 6, 737-748 (2012). 
27. K. K. Chow, S. Yamashita, and Y. W. Song, "A widely tunable wavelength converter based on nonlinear polarization rotation in a carbon-nanotube-deposited D-shaped fiber," Opt. Express 17, 7764-7669 (2009).

28. K. K. Chow, and S. Yamashita, "Four-wave mixing in a single-walled carbonnanotube-deposited D-shaped fiber and its application in tunable wavelength conversion," Opt. Express 17, 15608-15613 (2009).

29. P. Dumais, F. Gonthier, S. Lacroix, J. Bures, A. Villeneuve, P. G. J. Wigley, and G. I. Stegeman, "Enhanced self-phase modulation in tapered fibers," Opt. Lett. 18, 19961998 (1993).

30. W. Li, B. Chen, C. Meng, W. Fang, Y. Xiao, X. Li, Z. Hu, Y. Xu, L. Tong, H. Wang, W. Liu, J. Bao, and Y. R. Shen, "Ultrafast all-optical graphene modulator," Nano Lett. 14, 955-959 (2014).

31. J. F. Li, H. Y. Luo, Y. L. He, Y. Liu, L. Zhang, K. M. Zhou, A. G. Rozhin, and S. K. Turistyn, "Semiconductor saturable absorber mirror passively Q-switched $2.97 \mu \mathrm{m}$ fluoride fiber laser," Laser Phys. Lett. 11, 065102 (2014).

32. K. R. Khan, T. X. Wu, D. N. Christodoulides, and G. I. Stegeman, "Soliton switching and multi-frequency generation in a nonlinear photonic crystal fiber coupler," Opt. Express 16, 9417-9428 (2008).

33. F. AbdelMalek, H. Li, A. Schulzgen, J. V. Moloney, N. Peyghambarian, H. Ademgil, and S. Haxha, "A nonlinear switch based on irregular structures and nonuniformity in doped photonic crystal fibers," IEEE J. Quantum Electron. 45, 684-693 (2009). 
34. M. Bajcsy, S. Hofferberth, V. Balic, T. Peyronel, M. Hafezi, A. S. Zibrow, V. Vuletic, and M. D. Lukin, "Efficient all-optical switching using slow light within a hollow fiber," Phys. Rev. Lett. 102, 203902 (2009).

35. S. Maguis, G. Laffont, P. Ferdinand, B. Carbonnier, K. Khan, T. Mekhalif, and M. C. Millot, "Biofunctionalized tilted fiber Bragg gratings for label-free immunosensing," Opt. Express 16, 19049-19062 (2008).

36. F. Liu, T. Guo, J. Liu, X. Zhu, Y. Liu, B. O. Guan, and J. Albert, "High-sensitive and temperature-self-calibrated tilted fiber grating biological sensing probe," Chin. Sci. Bull. 58, 2611-2615 (2013).

37. T. Guo, F. Liu, Y. Liu, N. Chen, B. O. Guan, and J. Albert, "In-situ detection of density alteration in non-physiological cells with polarimetric tilted fiber grating sensors," Biosens. Bioelectron. 55, 452-458 (2014).

38. J. Zheng, X. Dong, P. Zu, L. Shao, C. C. Chan, Y. Cui, and P. P. Shum, "Magnetic field sensor using tilted fiber grating interacting with magnetic fluid," Opt. Express 21, 17863-17868 (2013).

39. Y. Shevchenko, T. J. Francis, D. A. D. Blair, R. Walsh, M. C. DeRosa, J. Albert, "In situ biosensing with a surface plasmon resonance fiber grating aptasensor," Anal. Chem. 83, 7027-7034 (2011).

40. G. E. Villanueva, M. B. Jakubinek, B. Simard, C. J. Oton, J. Matres, L. Y. Shao, P. P. Millan, J. Albert, "Linear and nonlinear optical properties of carbon nanotube- coated single-mode optical fiber gratings," Opt. Lett. 36, 2104-2016 (2011).

41. L. Y. Shao, M. B. Jakubinek, T. Sun, B. Simard, J. Albert, "Four-wave mixing in carbon nanotube-coated optical fiber gratins," Appl. Phys. Lett. 100, 071108 (2012). 
42. F. Liu, J. Albert, "40 GHz-rate all-optical cross-modulation of core-guided near infrared light in single mode fiber by surface plasmons on gold-coated tilted fiber Bragg gratins," APL Photonics 4, 126104 (2019).

43. D. Taverner, D. G. R. Broderick, D. J. Richardson, R. I. Laming, and M. Ibsen, "Nonlinear self-switching and multiple gap-soliton formation in a fiber Bragg grating," Opt. Lett. 23, 328-330 (1998).

44. N. G. R. Broderick, D. Taverner, and D. J. Richardson, "Nonlinear switching in fibre Bragg gratings," Opt. Express 3, 447-453 (1998).

45. N. G. R. Broderick, D. J. Richardson, and M. Ibsen, "Nonlinear switching in a 20-cmlong fiber Bragg grating," Opt. Lett. 25, 536-538 (2000).

46. T. Guo, L. Shao, H. Y. Tan, P. A. Krug, and J. Albert, "Tilted fiber grating accelerometer incorporating and abrupt biconical taper for cladding to core recoupling," Opt. Express 17, 20651-20660 (2009).

47. C. Yeh, and G. Lindgren, "Computing the propagation characteristics of radially stratified fibers: An efficient method," Appl. Opt. 16, 483-493 (1977).

48. M. Z. Alam, and J. Albert, "Selective excitation of radially and azimuthally polarized optical fiber cladding modes," J. Lightw. Technol. 31, 3167-3175 (2013).

49. W. J. Zhou, Y. Zhou, and J. Albert, "A true fiber optic refractometer," Laser Photonics Rev 11, 1600157 (2017).

50. Y. Shevchenko, C. Chen, M. A. Dakka, J. Albert, "Polarization-selective grating excitation of plasmons in cylindrical optical fibers," Opt. Lett. 35 (5), 637-639 (2010). 
51. W. Zhou, D. J. Mandia, S. T. Barry, and J. Albert, "Absolute near-infrared refractometry with a calibrated tilted fiber Bragg grating," Opt. Lett. 40 (8), 1713-1716 (2015).

52. L. Y. Shao, M. J. Yin, H. Y. Tan, and J. Albert, "Fiber optic pH sensor with selfassembled polymer multilayer nanocoatings," Seonsors 13, 1425-1434 (2013).

53. A. L. Aldaba, A. G. Vila, M. Debliquy, M. L. Amo, C. Caucheteur, D. Lahem, "Polyaniline-coated tilted fiber Bragg gratings for $\mathrm{pH}$ sensing," Sens. Actuators B 254, 1087-1093 (2018).

54. Y. Zhang, F. Wang, Z. Liu, Z. Duan, W. Cui, J. Han, Y. Gu, Z. Wu, Z. Jing, C. Sun, and W. Peng, "Fiber-optic anemometer based on single walled carbon nanotube coated filted fiber Bragg grating,” Opt. Express 25, 24521-24530 (2017).

55. B. Jiang, X. Lu, X. Gan, M. Qi, Y. Wang, L. Han, D. Mao, W. Zhang, Z. Ren, and J. Zhao, "Graphene-coated tilted fiber-Bragg grating for enhanced sensing in lowrefractive-index region,” Opt. Lett. 40, 3994-3997 (2015).

56. V. Voisin, J. Pilate, P. Damman, P. Megret, and C. Caucheteur, "Highly sensitive detection of molecular interactions with plasmonic optical fiber grating sensors," Biosens. Bioelectron. 51, 249-254 (2014).

57. T. Guo, F. Liu, X. Liang, X. H. Qiu, Y. Y. Huang, Ch. Xie, P. Xu, W. Mao, B. O. Guan, and J. Albert, "Highly sensitive detection of urinary protein variations using tilted fiber grating sensors with plasmonic nanocoatings," Biosens. Bioelectron. 78, 221-228 (2016).

58. Y. Yuan, T. Guo, X. H. Qiu, J. H. Tang, Y. Y. Huang, L. Zhuang, Sh. G. Zhou, Zh. H. Li, B. O. Guan, X. M. Zhang, and J. Albert, "Electrochemical surface plasmon 
resonance fiber-optic sensor: In situ detection of electroactive biofilms," Anal. Chem. 88, 7609-7616 (2016).

59. Z. C. Zhang, T. Guo, X. J. Zhang, J. Xu, W. P. Xie, M. Nie, Q. Wu, B. O. Guan, and J. Albert, "Plasmonic fiber-optic vector magnetometer," Appl. Phys. Lett. 108, 101105 (2016).

60. C. Caucheteur, T. Guo, F. Liu, B. O. Guan, and J. Albert, "Ultrasensitive plasmonic sensing in air using optical fibre spectral combs," Nat. Commun. 7, 13371 (2016).

61. X. J. Zhang, Sh. Sh. Cai, F. Liu, H. Chen, P. G. Yan, Y. Yuan, T. Guo, and J. Albert, "In situ determination of the complex permittivity of ultrathin H2-infused palladium coatings for plasmonic fiber optic sensors in the near infrared," J. Mater. Chem. C 6, $5161-5170$ (2018).

62. J. J. Lao, P. Sun, F. Liu, X. J. Zhang, Ch. X. Zhao, W. J. Mai, T. Guo, G. Zh. Xiao, and J. Albert, "In situ plasmonic optical fiber detection of the state of charge of supercapacitors for renewable energy storage," Light Sci. Appl. 7, 34 (2018).

63. J. B. Khurgin, "Ultimate limit of field confinement by surface plasmon polaritons," Faraday Discuss. 178, 109-122 (2015).

64. C. Caucheteur, V. Voisin, and J. Albert, "Near-infrared grating-assisted SPR optical fiber sensors: design rules for ultimate refractometric sensitivity," Opt. Express 23, 2918-2932 (2015).

65. E. Kretschmann, "The determination of optical constants of metals by excitation of surface plasmons," Z. Phys. 241, 313-324 (1971).

66. J. R. Lakowicz, "Radiative decay engineering: biophysical and biomedical applications," Anal. Biochem. 298, 1-24 (2001). 
67. H. A. Atwater, and A. Polman, "Plasmonic for improved photovoltaic devices," Nature Mater. 9, 205-213 (2010).

68. H. Baida, D. Mongin, D. Christofilos, G. Bachelier, A. Cru, P. Maioli, N. D. Fatti, and F. Vallee, "Ultrafast nonlinear optical response of a single gold nanorod near its surface plasmon resonance," Phys. Rev. Lett. 107, 057402 (2011).

69. A. Lanoul, M. Robson, V. Pripotnev, and J. Albert, "Polarization-selective excitation of plasmonic resonance in silver nanocube random arrays by optical fiber cladding mode evanescent fields," RSC Adv. 4, 19725-19730 (2014).

70. S. Lepinay, A. Staff, A. Ianoul, and J. Albert, "Improved detection limits of protein optical biosensors coated with gold nanoparticles," Biosens. Bioelectron. 52, 337-344 (2014).

71. G. V. Hartland, "Optical studies of dynamics in noble metal nanostructures," Chem. Rev. 111, 3858-3887 (2011).

72. C. Noguez, "Surface plasmon on metal nanoparticles: the influence of shape and physical environment," J. Phys. Chem. C 111, 3806-3819 (2007).

73. D. Gall, "Electron mean free path in elemental metals," J. Appl. Phys. 119, 085101 (2016).

74. H. Chen, L. Shao, Q. Li, and J. Wang, "Gold nanorods and their plasmonic properties," Chem. Soc. Rev. 42, 2679-2724 (2013).

75. G. A. Wurtz, R. Pollard, W. Hendren, G. P. Wiederrech, D. J. Gosztola, V. A. Podolskiy, and A. V. Zayats, "Designed ultrafast optical nonlinearity in a plasmonic nanorod metamaterial enhanced by nonlocality," Nat. Nanotechnol. 6, 107-111 (2011). 
76. M. Celebrano, X. Wu, M. Baselli, S. Grobmann, P. Biagioni, A. Locatelli, C. D. Angelis, G. Cerullo, R. Osellame, B. Hecht, L. Duo, F. Ciccacci, and M. Finazzi, "Mode matching in multiresonant plasmonic nanoantennas for enhanced second harmonic generation," Nat. Nanotechnol. 10, 412-417 (2015).

77. V. Giannini, A. T. F. Dominguez, S. C. Heck, and S. A. Maier, "Plasmonic nanoantennas: fundamentals and their use in controlling the radiative properties of nanoemitters," Chem. Rev. 111, 3888-3912 (2011).

78. Z. P. Qin and J. C. Bischof, "Thermophysical and biological responses of gold nanoparticle laser heating," Chem. Soc. Rev. 41, 1191-1217 (2012).

79. Y. W. Hao, H. Y. Wang, Y. Jiang, Q. D. Chen, K. Ueno, W. Q. Wang, H. Misawa, and H. B. Sun, "Hybrid-state dynamics of gold nanorods/dye J-aggregates under strong coupling," Angew. Chem. Int. Ed. 50, 7824-7828 (2011).

80. S. Link, M. B. Mohamed, and M. A. El-Sayed, "Simulation of the optical absorption spectra of gold nanorods as a function of their aspect ratio and the effect of the medium dielectric constant," J. Phys. Chem. B 103, 3073-3077 (1999).

81. C. A. Foss, J. G. L. Hornyak, J. A. Stockert, and C. R. Martin, "Template-Synthesized Nanoscopic Gold Particles: Optical Spectra and the Effects of Particle Size and Shape," J. Phys. Chem. 98, 2963-2971 (1994).

82. J. Aizpurua, G. W. Bryant, L. J. Richter, and F. J. G. D. Abojo, "Optical properties of coupled metallic nanorods for field-enhanced spectroscopy," Phys. Rev. B 71, 235420 (2005).

83. M. Pelton, and G. Bryant, Introduction to metal-nanoparticle plasmonics. (Wiley, 2013). 
84. A. Bialiayeu, A. Bottomley, D. Prezgot, A. Ianoul, and J. Albert, "Plasmon-enhanced refractometry using silver nanowire coating on tilted fibre Bragg gratings," Nanotechnology 23, 444012 (2012).

85. J. M. Renoirt, M. Debliquy, J. Albert, A. Ianoul, and C. Caucheteur, "Surface plasmon resonances in oriented silver nanowire coatings on optical fibers," J. Phys. Chem. C 118, 11035-11042 (2014).

86. H. B. Liao, R. F. Xiao, J. S. Fu, P. Yu, G. K. L. Wong, and P. Sheng, "Large thirdorder optical nonlinearity in $\mathrm{Au}: \mathrm{SiO}_{2}$ composite films near the percolation threshold," Appl. Phys. Lett. 70, 1 (1997).

87. E. Xenogiannopoulou, K. Iliopoulos, S. Couris, T. Karakouz, A. Vaskevich, and I. Rubinstein, "Third-order nonlinear optical response of gold-island films," Adv. Funct. Mater. 18, 1281-1289 (2008).

88. P. Gadenne, F. Brouers, V. M. Shalaev, A. K. Sarychev, "Giant stokes fields on semicontinuous metal films," J. Opt. Soc. Am. B 15, 68-72 (1998).

89. M. Breit, V. A. Podolskiy, S. Gresillon, G. V. Plessen, J. Feldmann, J. C. Rivoal, and P. Gadenne, "Experimental observation of percolation-enhanced nonlinear light scattering from semicontinuous metal films," Phys. Rev. B 64, 125106 (2001).

90. M. I. Stockman, D. J. Bergman, C. Anceau, S. Brasselet, and J. Zyss, "Enhanced second-harmonic generation by metal surfaces with nanoscale roughness: nanoscale dephasing, depolarization, and correlations," Phys. Rev. Lett. 92, 057402 (2004).

91. S. D. Zuani, T. Peterseim, A. Berrier, B. Gompf, and M. Dressel, "Second harmonic generation enhancement at the percolation threshold," Appl. Phys. Lett. 104, 241109 (2014). 
92. P. Gadenne, Y. Yagil, and G. Deutscher, "Transmittance and reflectance in situ measurements of semi-continuous gold films during deposition," J. Appl. Phys. 66, 3019- 3025(1989).

93. S. Ding, X. Wang, D. J. Chen, and Q. Q. Wang, "Optical percolation and nonlinearity of sputtered Ag island films," Opt. Express 14, 1541-1546 (2006).

94. L. Y. Shao, J. P. Coyle, S. T. Barry, and J. Albert, "Anomalous permittivity and plasmon resonances of copper nanoparticle conformal coatings on optical fibers," Opt. Express 1, 128-137 (2011).

95. W. Zhou, D. J. Mandia, M. B. E. Griffiths, A. Bialiayeu, Y. Zhang, P. G. Gordon, S. T. Barry, and J. Albert, "Polarization-dependent properties of the cladding modes of single mode fiber covered with gold nanoparticles," Opt. Express 21, 245-255 (2013).

96. W. Zhou, D. J. Mandia, M. B. E. Griffiths, S. T. Barry, and J. Albert, "Effective permittivity of ultrathin chemical vapor deposited gold films on optical fibers at infrared wavelength," J. Phys. Chem. C 118, 670-678 (2014).

97. A. Bialiayeu, C. Caucheteur, N. Ahamad, A. Ianoul, and J. Albert, "Self-optimized metal coating for fiber plasmonics by electroless deposition," Opt. Express 19, 1874218753 (2011).

98. W. Zhou, D. J. Mandia, S. T. Barry, and J. Albert, "Anisotropic effective permittivity of an ultrathin gold coating on optical fiber in air, water, and saline solutions," Opt. Express 22, 31665-31676 (2014).

99. D. J. Mandia, W. Zhou, M. J. Ward, H. Joress, J. J. Sims, J. B. Giorgi, J. Albert, and S. T. Barry, "The effect of ALD-grown Al2O3 on the refractive index sensitivity of CVD gold-coated optical fiber sensors," Nanotechnology 26, 434002 (2015). 


\title{
Chapter 2: Optical detection of the percolation threshold of nanoscale silver coatings with optical fiber gratings
}

\begin{abstract}
The metal-to-dielectric transition of silver films deposited on single-mode optical fibers is monitored by measurements of the transmission spectra of tilted fiber Bragg gratings inscribed in the core of the fiber. In-situ, real-time measurements of the spectrum at wavelengths near $1550 \mathrm{~nm}$ during the wet etching of a $50 \mathrm{~nm}$ thick silver coating show a sudden and temporary decrease of more than $90 \%$ in the amplitudes of the core-to-cladding mode coupling resonances when the film thickness reaches $18 \mathrm{~nm}$. Confirmation that this observation corresponds to the percolation threshold is obtained from simultaneous measurements of the silver coating conductivity during etching and by simulations of the grating response. The characteristic spectral signature of the percolation threshold is only obtained for cladding modes polarized azimuthally in the fiber crosssection, i.e. parallel to the film surface.
\end{abstract}

\subsection{Introduction}

Following early investigations in the 1950s, ultra-thin metallic and semi-metallic coatings have received renewed interest in recent years due to their interesting plasmonic and nonlinear optical properties [1-16]. Part of the interest also lies in the fact that these properties depend quite strongly on how the coating is produced (method, rate, substrate) $[1,5]$. Since thicknesses of interest often lie in the few tens of nm range, and since such coatings tend to have some roughness, standard optical techniques to measure their complex permittivity from visible to near infrared wavelengths $(400-1600 \mathrm{~nm})$, such as spectroscopic ellipsometry, are pushed to their limits $[17,18]$. This is further complicated 
by the fact that the response of ultrathin metals depends greatly on the orientation of the electric field of an incoming electromagnetic wave relative to the plane of the film, since the in-plane conductivity can be much larger than the out-of-plane one [19]. Finally, there have been efforts to fabricate metal films with thicknesses as near as possible to the percolation threshold, i.e. where the in-plane conductivity changes from near zero to significant values marking the metal-to-insulator transition and where the absorption at visible and near-infrared wavelengths becomes anomalous [7-8,20]. Also, at the percolation threshold the real part of the "metal" film permittivity goes from highly negative to slightly positive and the zero crossing corresponds to a situation where the nonlinear optical properties are greatly enhanced [9-13]. Since this transition occurs very suddenly as a function of thickness and that it depends strongly on the material morphology, it is difficult to prepare films exactly at the percolation threshold even with a thickness monitor. Here, we present a highly efficient method to determine the percolation threshold of silver layers deposited on silica surfaces independently of their thickness, based on the polarization-controlled measurements of high Q-factor plasmonic resonant structures at near infrared wavelengths using low loss tilted fiber Bragg gratings (TFBG) written in standard single mode optical fibers [21-22]. The overall design of the experiment is shown in Fig. 2.1. It will be shown that the transmission spectra of TFBGs with a $50 \mathrm{~nm}$ thick silver coating have a clear, sudden, and unique collapse of the grating resonance amplitudes of more than $90 \%$ during a slow wet etch of the silver when its thickness drops below $\sim 20 \mathrm{~nm}$. Independent measurements of the coating conductivity during the etching further demonstrates that these thicknesses also correspond to the metal-insulator transition with a sudden change in resistance from less than 0.1 to over $10 \mathrm{M} \Omega$ across a length of 2.3 
$\mathrm{cm}$ on the surface of the fiber. Therefore, the proposed technique allows the real-time and in-situ monitoring of the preparation of ultrathin metallic films at the percolation threshold, either as a process measurement tool based on plasmon excitation [23-27], or by itself for the fabrication of optical fiber-based devices with enhanced nonlinear optical properties [28-30]. The importance of such technique is that the percolation transition is very abrupt (in terms of rate of change with thickness) and that the properties of ultrathin films depend greatly on the process used and on the rate of deposition (or etching), and therefore an insitu measurement is critical to ensure that the film fabrication is stopped exactly at the threshold condition [1,8-9].

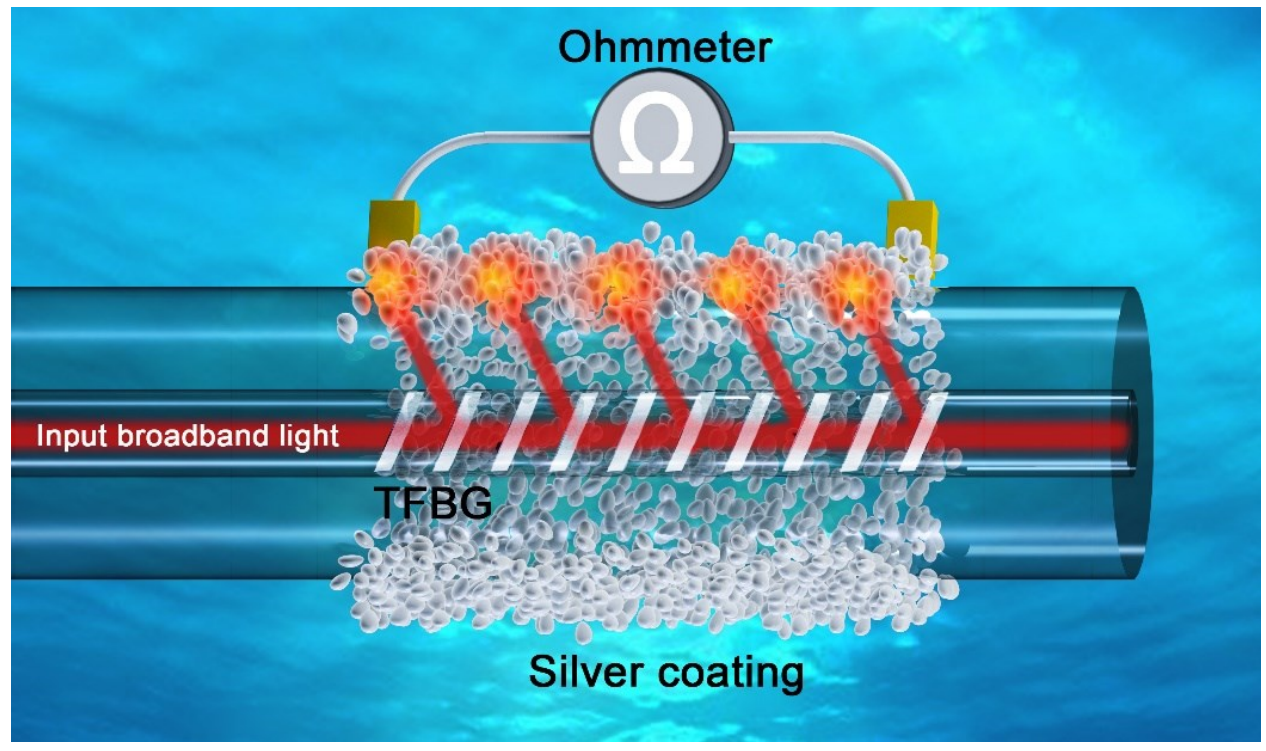

Fig. 2.1. Sketch of the experimental configuration used to measure transmission of silver coated TFBGs. The device is fully immersed in a dilute etching solution and the input and output leads of the fiber are connected to an external polarization-controlled broadband source (BBS) and optical spectral analyzer (OSA) respectively. For the confirmation of the correlation between the optical measurements and the silver conductivity presented in this work, additional probes from an electrical resistance meter are attached to the silver coating. See Methods for details. 


\subsection{Results and discussion}

Transmission spectra of silver coated TFBGs in the etch solution are shown in Fig. 2.2, at the beginning and end of the etch process, as well as at a critical intermediate step. The spectra for both polarization states before the etch clearly show that the silver coating is metallic: the P-polarized spectrum has a notch in the spectrum of resonances near $1550 \mathrm{~nm}$ due to coupling of the cladding modes to a surface plasmon polariton of the silver-water interface, forming a surface plasmon resonance (SPR) superimposed on the cladding mode resonances [22]. The initial thickness of $50 \mathrm{~nm}$ is very close to the optimal thickness for observation of SPR effects in silver on glass substrates, but for P-polarized light only [31]. At a characteristic time of 27 minutes of etching, the thickness has decreased to a point where the SPR has broadened to cover the $1560-1590 \mathrm{~nm}$ range, as expected for thinner, rougher metallic coatings [31]. Upon further etching, the P-spectrum ends up similar to that of a bare TFBG in water, with a relatively sharp decrease in the amplitudes of the cladding mode resonances at wavelengths shorter than those of the last cladding guided resonance, where modes become leaky and hence lossy [21]. In the remainder of the text we will refer to the etch solution as water since it is so dilute that its optical properties are essentially identical. On the other hand, the resonances in S-polarization present strikingly different behavior. Before etching, the metal coating completely shields the guided modes from the external medium and even resonances with effective indices lower than that of water remain essentially lossless and guided [32]. Then, as the etch progresses, the resonance amplitudes in S-spectra begin to fade more or less uniformly, reaching a point after 170 minutes where they are completely collapsed, leaving a smooth and slowly varying attenuation across the measurement range (except near the Bragg wavelength 
where the modes are either confined to the core or very strongly confined cladding modes). At the end of the etch, the S-spectrum becomes similar to that of the P-spectrum, for the same reasons (i.e. the behavior of a bare TFBG in water) [33].
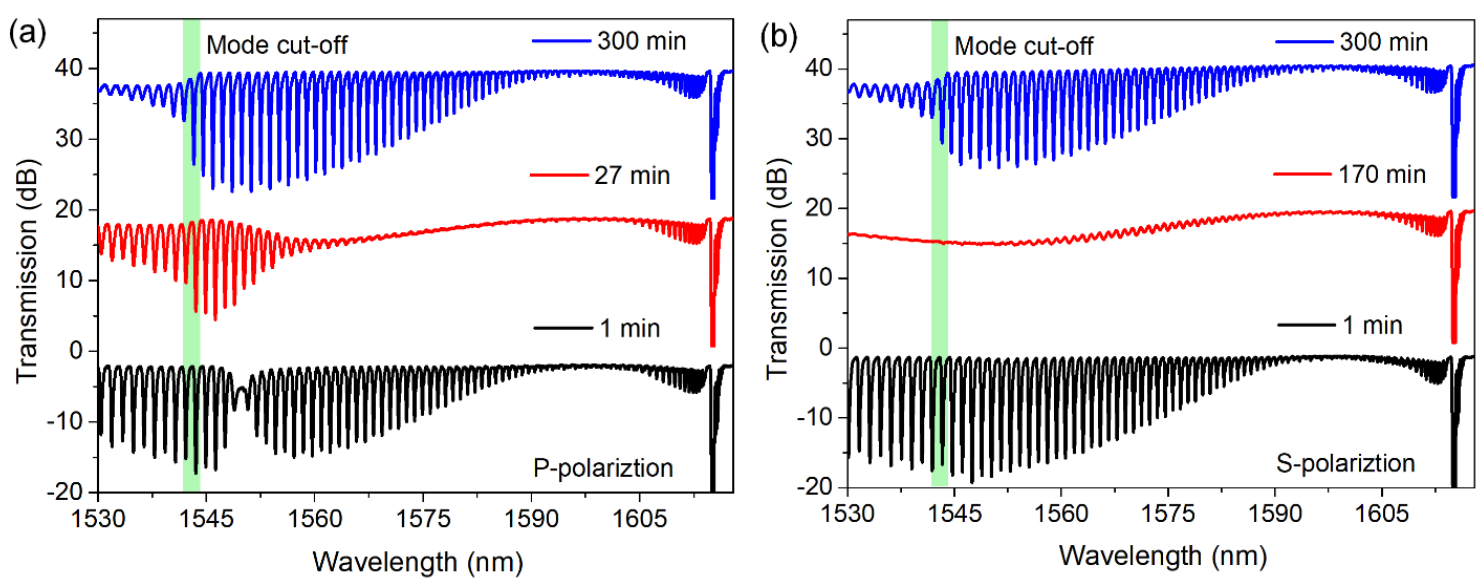

Fig. 2.2. Representative experimental transmission spectra at various characteristic stages of etching for: (a) radially polarized cladding modes; (b) azimuthally polarized cladding modes. The three spectra in each plot are offset for clarity. The green shaded spectral range corresponds to the guided-to-leaky mode transition, or "mode cut-off".

In order to describe what happens to the cladding modes, the surface morphology of silver coated TFBGs was measured by atomic force microscopy (AFM) on samples etched for various durations. Fig. 2.3 and 2.4 show the surface profiles obtained and the extracted average thickness and roughness as a function of etch time, respectively. 

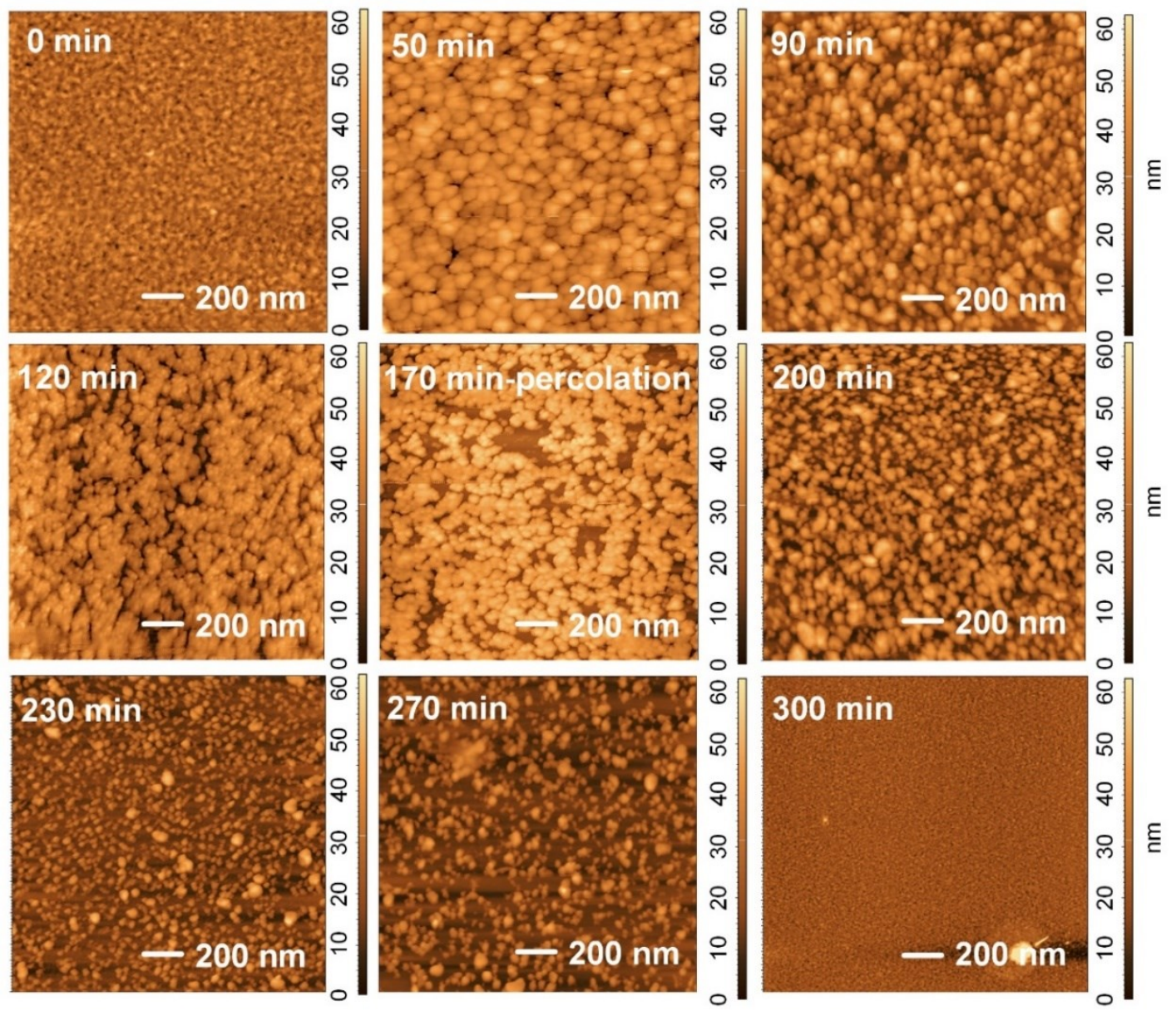

Fig. 2.3. AFM of the surface morphology on nominally identical silver coated TFBGs with an initial silver thickness of $50 \mathrm{~nm}$ and etched for various durations (indicated by the labels). 


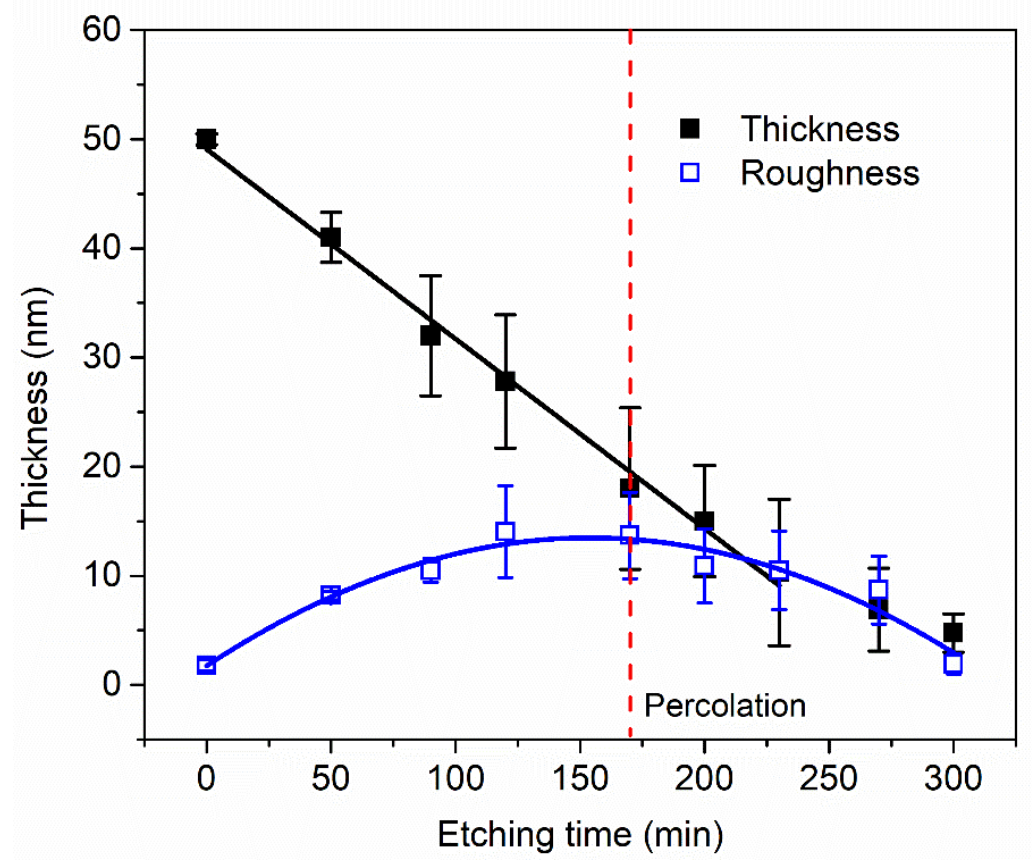

Fig. 2.4. Measured average thickness and roughness from the atomic force microscopy results of Fig. 2.3. A linear fit of the thicknesses between 0 and 230 minutes is used to provide a calibration of the thickness vs etch time correspondence (over this range) for the results of chapter 2.

It is clear from Fig. 2.3 and 2.4 that the silver coatings are quite uniform and smooth initially but that as the etch progresses, the thickness decreases regularly but the roughness peaks at significant values (relative to the thickness) between 125 and 175 minutes, corresponding to thicknesses between 18 to $27 \mathrm{~nm}$. A polynomial fit of the thicknesses on Fig. 2.4 was used to calibrate the correspondence between etch time and thickness for all the spectral results in the remainder of the text. Another interesting observation from the results of Fig. 2.3 occurs in the image corresponding to an etch of 170 minutes where a very discontinuous structure with significant agglomerations of very uniform nanoparticles and large voids is found, unlike all other etch times. In order to further elucidate the correlation between the optical and physical measurements, more detailed spectral evolution plots as a function of etch time are presented in Fig. 2.5. 


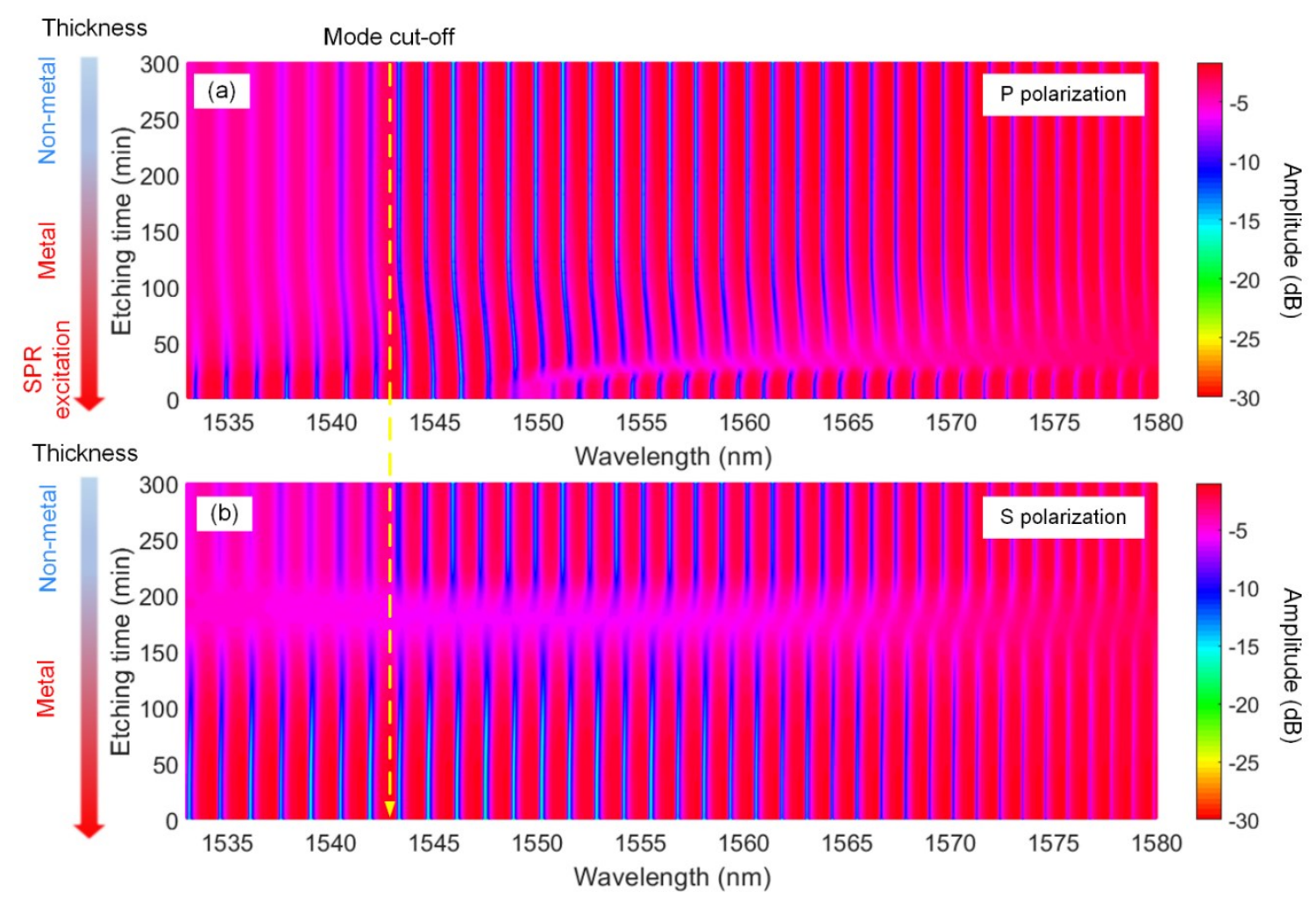

Fig. 2.5. Continually measured spectra during etching for the two polarization states, at intervals of 1.5 minutes (Video 1 in Supporting Information). Resonances show up as blue lines of various hues except when they diminish significantly in amplitudes, tending towards pink colours. The vertical dashed lines in the figure indicate the approximate mode cutoff point where the mode effective indices become smaller than 1.3154 (the refractive index of water at these wavelengths).

While there is interesting information throughout the plots shown in Fig. 2.5, the spectral region where the strongest resonances occur is between 1545 and $1565 \mathrm{~nm}$, and it is also where the most clearly identifiable changes are found during etching. At longer wavelengths, the evanescent fields of the modes penetrate less and less outside of the fiber cladding and therefore become insensitive to what happens in the silver layer. At shorter wavelengths the modes have effective indices lower than that of the external medium and as soon as the silver layer becomes thin enough some of the evanescent fields tunnel and 
lose energy radiatively, in addition to the intrinsic losses due to the silver layer itself. Concentrating thereon on the resonances of interest, the P-spectra indicate a widening and weakening of the SPR notch as the thickness decreases, in total accord with the literature on the subject [31], followed by a gradual transition towards the spectrum corresponding of a bare fiber in water. By the time the thickness reaches the "interesting" range near 20 $\mathrm{nm}$, there is so very little interaction between the radially polarized mode fields and the free electrons in the metal that the modes behave more or less as if the coating was not there: the mean free path of the electrons at these wavelengths becomes similar to the available path length across the layer thickness. For the S-spectra however, little change occurs until about 160 minutes of etching and then a sudden, large decrease in the mode amplitudes is observed simultaneously across the band, followed by another sudden recovery associated with a wavelength shift. Because of this highly peculiar behavior, and recalling that the S-spectra correspond to cladding modes with their electric fields polarized in the plane of the coating and thus that the resonances probe the in-plane conductivity of the silver coating, the remainder of this chapter deals solely with S-polarized results. Having explored the "big picture", we now look more closely at one of the interesting resonances from Fig. 2.5. Fig. 2.6 shows how the amplitude (i.e. minimum power level) and the wavelength of the resonance initially located at $1544.8 \mathrm{~nm}$ evolves during etching. 


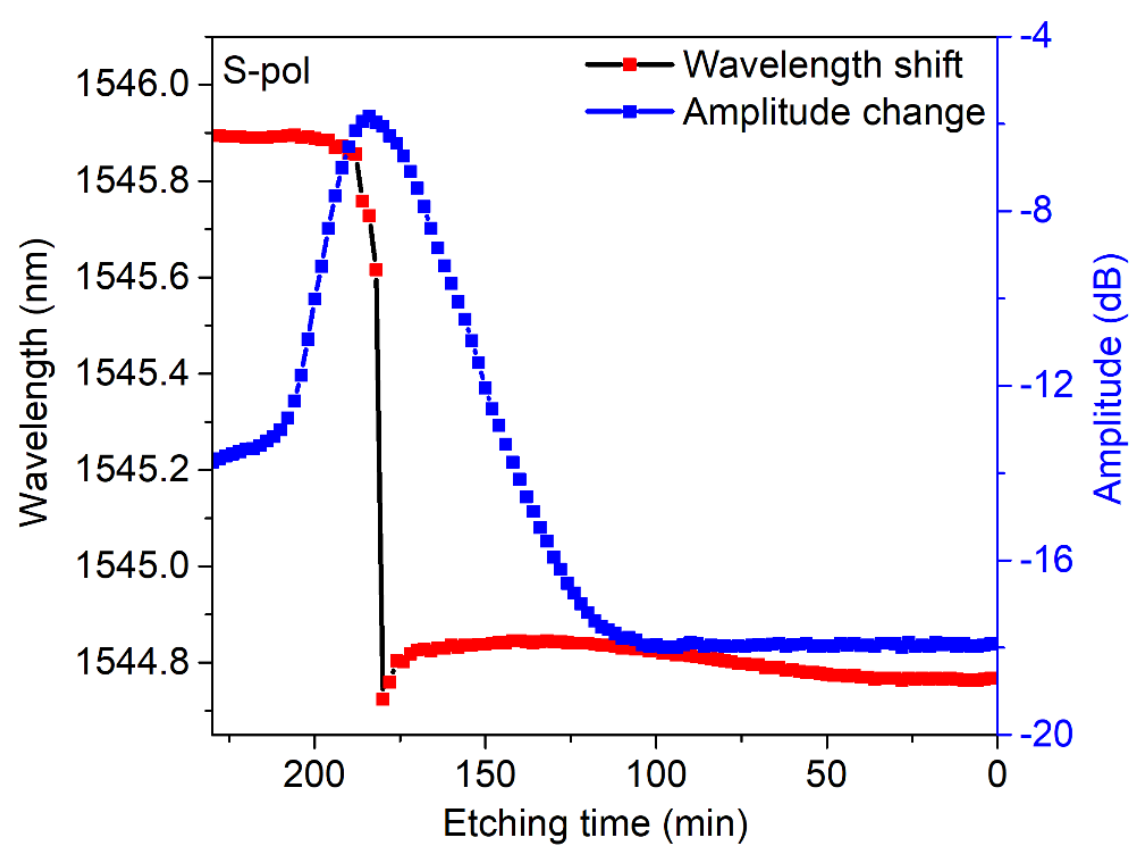

Fig. 2.6. Extraction of the experimental wavelength and amplitude of one of last non-leaky resonances as a function of etch time (the etch time axis is inverted so that the thicknesses increase from left to right).

Clearly, something special happens around 175-180 minutes (corresponding to a thickness of $18 \mathrm{~nm}$ ), where the amplitude change is maximum, and where the wavelength shift is large and very sudden. Since a decrease of the amplitude of a resonance (the measured transmission increases) and some broadening occurs with increasing mode loss, Fig. 2.6 indicate a loss increase (i.e. an increase in the imaginary part of the mode effective index), while the wavelength blue shift points to a large decrease in the real part of the mode index (to preserve phase matching between the wavelength and grating period) [21]. Before discussing this further, another test was carried out where electrical measurements were made simultaneously with optical ones at 2.5 -minute intervals. The results are presented in Fig. 2.7. In this case, the approximate sheet resistance is seen to remain near zero until about 155 minutes of etching ( $22 \mathrm{~nm}$ thickness), where it jumps to $300 \Omega / \square$ at 
160 minutes $(21 \mathrm{~nm})$. This is followed by a gradual increase to $1 \mathrm{k} \Omega / \square$ at 180 minutes (18 $\mathrm{nm}$ ) and then more rapidly to higher values as the thickness continues to decrease. If we assign the transition of the sheet resistance from 30 to $300 \Omega / \square$ to the metal-to-insulator transition, in accordance with the literature [16,34], the corresponding thickness would be $21 \mathrm{~nm}$ (with a roughness of $12 \mathrm{~nm}$ ). This value is different from the thickness of the transition observed in Fig. 2.6 (i.e. 18 nm), but we see in Fig. 2.7 that for this particular sample both the wavelength shift and amplitude collapse also occur earlier and correspond to the jump in resistance. The results of this simultaneous measurement, shown in Fig. 2.7, confirm two things. First, the "special point" where the amplitude collapse is maximum and where the resonance wavelength suddenly red-shifts by a large amount is a very good indicator of the metal-to-insulator transition and thus can be used (without the need for the electrical probing) to determine the percolation threshold of the film. Secondly, the percolation threshold occurs here at a slightly larger thickness $(21 \mathrm{~nm})$ than in the case shown in Fig. 2.6 where the thickness at the which the amplitude collapse was maximum at $18 \mathrm{~nm}$. This reflects the fact that there is no unique relationship between thickness and percolation threshold and that other factors come into play, causing the threshold to occur at slightly different thicknesses. The optical and electrical properties of these ultra-thin metal films depend strongly on the exact values of the process parameters and the very sudden nature of the transition makes it very hard to predict accurately the properties of films deposited in nominally identical conditions and at a given target thickness. Therefore, even an accurate in-situ thickness monitor may not be able to produce layers at the percolation threshold accurately and reproducibly. What is demonstrated here however, is not an in-situ measurement of thickness but rather of the percolation threshold itself. 


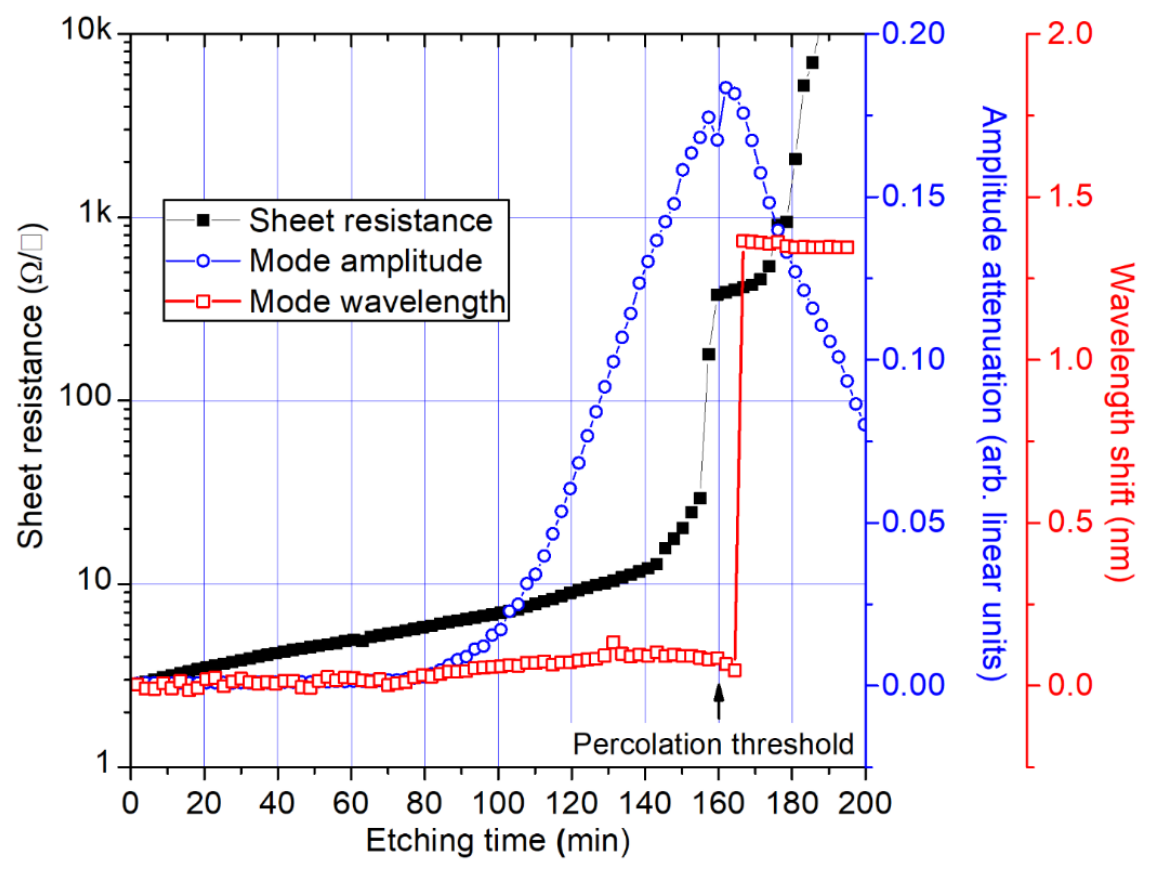

Fig. 2.7. Measurement of the silver coating sheet resistance during etching (filled black squares), with simultaneous measurements of the wavelength shift of the last guided resonance (empty red squares) and of its amplitude change (empty blue circles) (the amplitude change is reported here in linear units instead of $\mathrm{dB}$ ). The distance between the two electrical contacts on the coating was 2.3 $\mathrm{cm}$ (Video 2 in Supporting Information).

Finally, simulations of the TFBG transmission spectra were carried out to investigate if the observed results could be related to known properties of silver coatings at these thicknesses and wavelengths. S-polarized spectra were simulated for 253 silver different thicknesses between 0 and $50 \mathrm{~nm}$, over a wavelength range from 1544 to $1547 \mathrm{~nm}$. The real and imaginary parts of the refractive index of silver used in the simulations were taken from ref 2 , where similarly produced coatings were measured by spectroscopic reflection and transmission measurements at oblique incidence. The values they reported are reproduced in Fig. 2.8 and they were fitted between the measurements to allow continuous simulations with finer thickness spacing. 


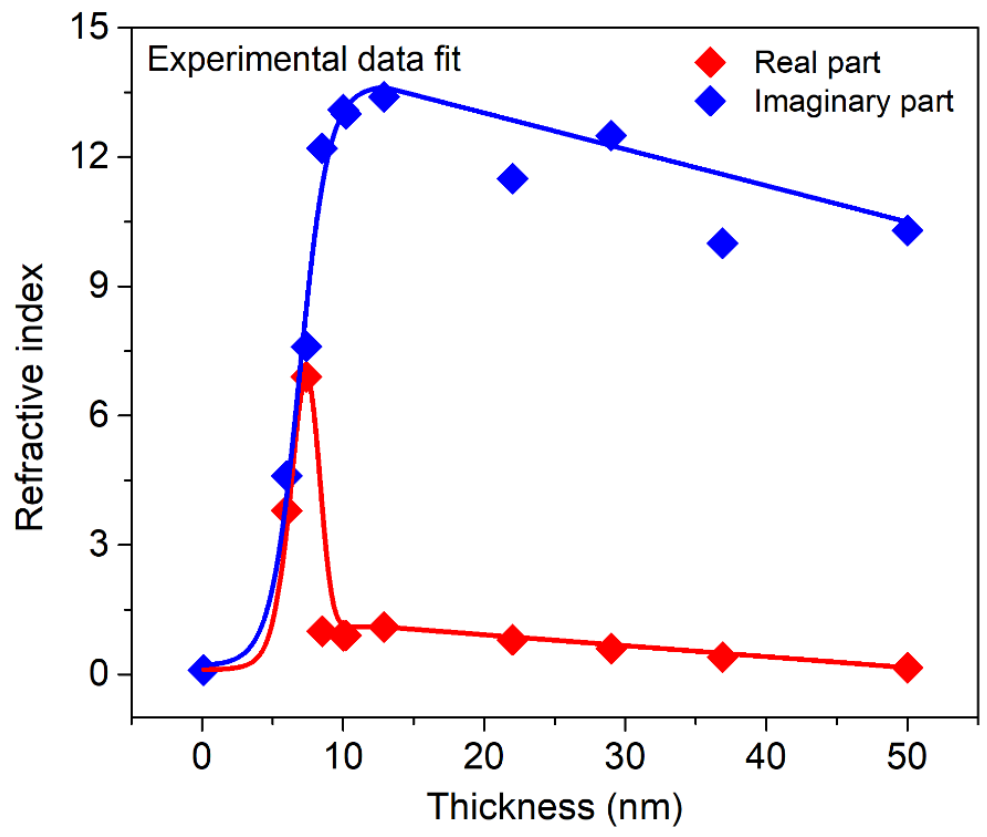

Fig. 2.8. Fitted values of silver refractive index as a function of thickness for thermally evaporated coatings and wavelengths near $1500 \mathrm{~nm}$ (data points reproduced from ref 2).

The percolation threshold shows up in Fig. 2.8 as a maximum in loss (imaginary part of the refractive index, k) and a sudden increase in the real part of the refractive index (n) which becomes nearly equal to the imaginary part (so that the real part of the relative permittivity $\left(\mathrm{n}^{2}-\mathrm{k}^{2}\right)$ goes from large and negative (typical of metals) to near zero. Using this data, Fig. 2.9 shows simulated spectra as well as replicas of the experimental results of Fig. 2.6, for both spectral evolution plots and relevant parameters. The main and obvious differences between our experimental results and those obtained by simulations with silver data from ref 2 are that the percolation threshold thickness is different (18 nm vs $6.5 \mathrm{~nm}$ ), the thickness range where the amplitude collapse is larger (20 nm vs $10 \mathrm{~nm}$ ), and amplitude collapse does not fully recover at the end of etching (while that of the simulation returns to that of a perfectly bare TFBG). On the other hand, the wavelength shifts overlap quite well in size and in the range of thicknesses over which they occur. 

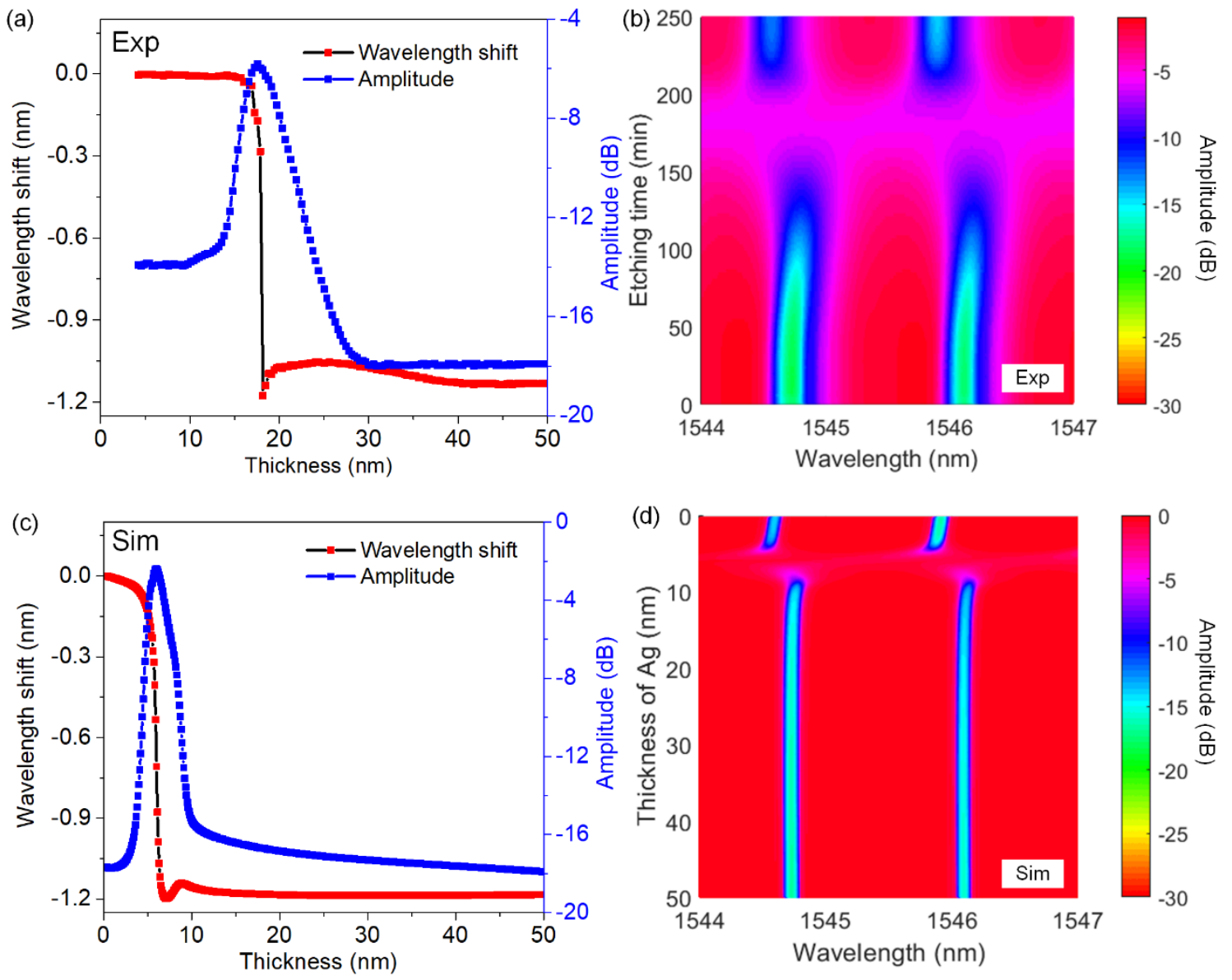

Fig. 2.9. Comparison between measured $(a, b)$ and simulated $(c, d)$ wavelength and amplitude shifts for a guided mode near cutoff. The properties of silver used in the simulation are taken from Fig. 2.8 (Video 3 in Supporting Information).

The overall qualitative agreement between the simulations and experiments supports our hypothesis that the sudden change in spectral properties occurring at thicknesses near 20 $\mathrm{nm}$ in our case is due to a metal-to-insulator transition. The large difference in thickness where this occurs in the simulation is obviously due to the fact that the percolation threshold of the underlying refractive index data (Fig. 2.8) is located at $6.5 \mathrm{~nm}$, and this is attributed to the fact that the silver coatings in the reference were produced by thermal evaporation, likely at a relatively fast rate (there is little information about this in the 
reference), and that measurements were performed on as-deposited films of different thicknesses. These conditions are known to produce very smooth and uniform films down to thicknesses below $10 \mathrm{~nm}[1,5]$. By contrast, while our silver coatings are very smooth and uniform initially (as seen in Fig. 2.3 and 2.4, a finding confirmed by the very narrow spectral width of the SPR coupling in Fig. 2.2 (a)), our method to obtain different thicknesses by etching a thicker film very slowly produces relatively rougher coatings. In fact, the roughness becomes equal to the thickness (within experimental error) after about 175 min of etching, i.e. in the vicinity of where we find the percolation thresholds and begin to lose conductivity. The roughness can also be the cause for the larger range of thicknesses where the amplitude collapse is observed experimentally. We attribute the widening of the loss curve to the fact that an additional loss mechanism, i.e. roughnessinduced scattering (which cannot be included in the imaginary part of the coating refractive index in the simulation because it does not change the permittivity in the same way as a change in conductivity), increases the overall mode loss for a range of thicknesses around the percolation threshold, including at the end of the etching. The latter effect is supported by the observation of residual silver particles with sizes near 1-3 nm in Fig. 2.3 (at 300 minutes of etching) and its extracted parameters in Fig. 2.4.

Finally, if the response of the TFBG to changes in silver thicknesses near the percolation threshold is due to the real part of the permittivity of the film approaching zero, it should be comparable to that of the same TFBG covered with a broadband perfect absorber, as discussed in ref 35 . In order to verify this, a bare TFBG was immersed in an index matching fluid, i.e. a liquid with an index equal to that of the cladding so that all the light coupled out of the core is radiated away instead of being coupled to cladding modes. Results from 
Fig. 2.10 show transmission spectra of a bare TFBG as a function of the refractive index of the external medium. As expected, and as measured from the transmitted light propagating in the core, individual resonances disappear completely and are replaced by a smooth spectral loss curve that only depends on the variation of the coupling coefficient of the grating with wavelength. This situation is identical to what would happen if a perfect absorber was located at the cladding boundary. Therefore, the similarity of the indexmatched spectrum of Fig. 2.10 to that of the silver coated-TFBG at the percolation point in Fig. 2.2(b) further supports our claim that the spectral signature is indicative of the percolation threshold.

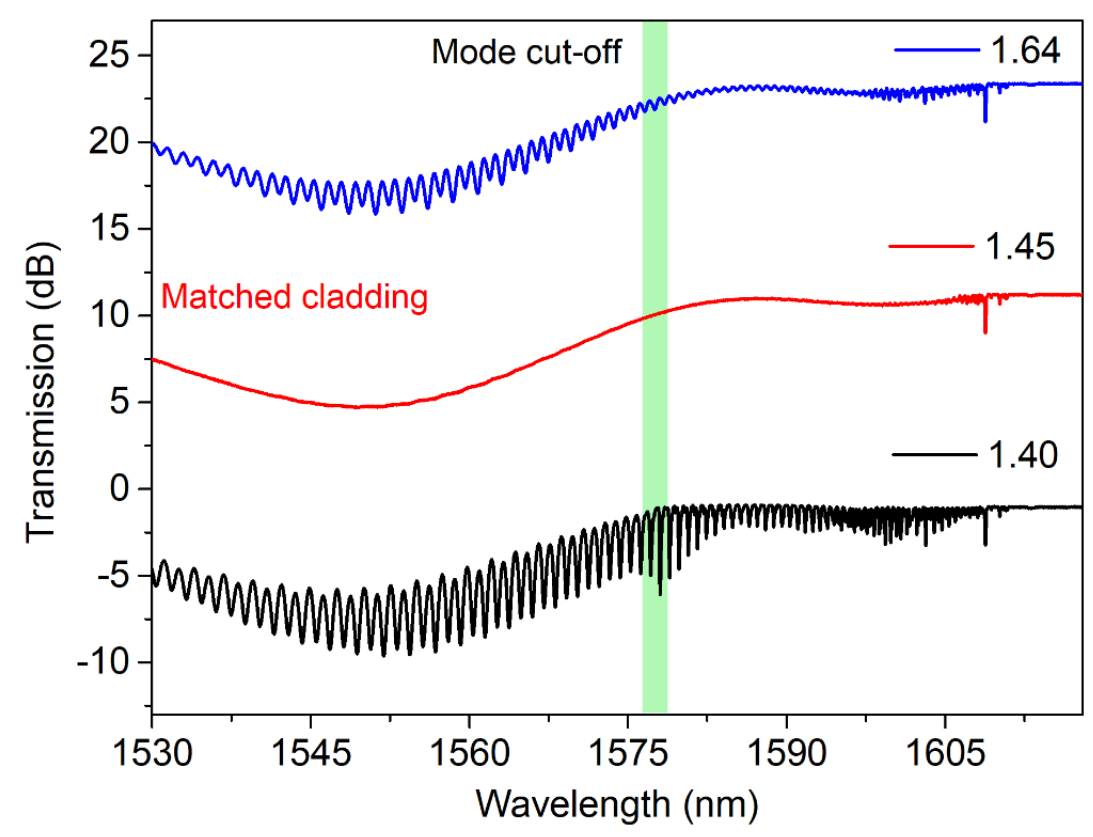

Fig. 2.10. Measured transmission spectra of a bare TFBG immersed in media with various refractive indices. The spectra are offset by $\sim 10 \mathrm{~dB}$ for clarity.

\subsection{Conclusion}

The occurrence of a percolation threshold for RF sputtered $50 \mathrm{~nm}$-thick silver films deposited on TFBGs and then slowly etched in liquid has been detected in-situ and in real 
time by simultaneous measurements of the TFBG transmission spectra. Polarizationselective spectral measurements for cladding modes with their electric fields aligned in the plane of the coating on the fiber surface show a sudden and very large change in the wavelengths (by more than $1 \mathrm{~nm}$, i.e. almost 10 times their width) and amplitudes (by more than $10 \mathrm{~dB}$ ) of a group of cladding modes coupled from the input core-guided light by the grating. It was verified by measurements of the electrical conductivity of the silver coating that this optical signature is associated with the beginning of a loss of conductivity, i.e. a percolation threshold. Simulations of the TFBG transmission confirm that a percolation threshold does lead to the types of spectral transmission changes observed experimentally, with only minor differences due to the exact values of the variation of the complex permittivity and scattering properties of silver coatings at thicknesses of the order of a few tens of nm. Furthermore, the spectral signature of the TFBG at the percolation point is identical to that of a TFBG with a perfect absorber at its boundary. This supports the claim that the silver coating has a near zero permittivity at this value of thickness. In contrast to other methods to prepare thin metal films exactly at the percolation threshold by measuring their thickness, TFBG measurements can precisely determine the moment where the layer goes through a metal-to-insulator (or insulator-to-metal) transition during deposition or etching instead of by trial and error using external measurements on multiple samples. These results indicate that TFBG technology is a valuable new approach to probe for the optical properties of matter at the nanoscale and that it can be used as a process monitor for both optical and electrical properties of ultrathin metal coatings on surfaces. 


\subsection{Materials and methods}

2.4.1 TFBG fabrication. Each $20 \mathrm{~mm}$-long TFBG with 12-degree internal tilt angle was inscribed in hydrogen-loaded CORNING SMF-28 fibers by using the phase-mask method and a rotation of the phase mask and the fiber around an axis perpendicular to the fiber orientation [21]. Hydrogen loading was performed at room temperature and a pressure of 15.2 MPa for 14 days. Gratings were written in a section of fiber stripped of its protective polymer jacket with a KrF pulsed excimer laser (model PM-848 from Light Machinery Inc.) emitting light at $248 \mathrm{~nm}$ and 100 pulses/s. A rectangular section of the large output beam of the laser was selected and cylindrically focused along the fiber axis over the desired length (at the fiber). The resulting pulse energy at the fiber was $\sim 40 \mathrm{~mJ}$. Optical transmission spectra were measured with an Erbium-doped fiber amplified emission source (ASE) and an optical spectrum analyzer (OSA) (ANDO AQ 6317B) with a resolution of $0.02 \mathrm{~nm}$. A polarization controller (OZ OPTICS. LTD.) was used to linearize the input light polarization either in the tilt plane of the TFBG (P-polarized) or out of plane (Spolarized), as determined by maximizing either the short or long wavelength resonance of the polarization-dependent pairs of resonances [36]. For the continuous monitoring of spectra during etching, the data acquisition system produced full spectra every 1.5 minutes.

2.4.2 Silver coating. $50 \mathrm{~nm}$-thick uniform silver films were deposited on TFBGs by RF magnetron sputtering (Polaron Instruments TRI-S500 fiber material metal coating system). To achieve a high-quality coating, the stripped fiber section was cleaned by immersing it in a piranha solution (an 8:1:1 mixture of deionized water, Ammonium Hydroxide, and Hydrogen Peroxide) for 30 minutes. Each TFBG was fixed on a movable holder inside the sputtering chamber and coated in $5 \mathrm{~Pa}$ pressure of Ar gas at a discharge current of $0.2 \mathrm{~A}$ 
and a voltage of $0.4 \mathrm{kV}$. The uniformity of the silver film around the cladding surface was ensured by rotating the fiber around its axis at a constant speed of $0.5 \mathrm{rad} / \mathrm{s}$, resulting in a mass-equivalent thickness of $50 \mathrm{~nm}$ in $130 \mathrm{~s}$ of deposition.

2.4.3 Silver etching. In order to be able to characterize the film state with sufficient thickness resolution and to obtain highly uniform thickness changes, the silver-coated TFBGs were etched in very dilute $(1: 10,000)$ solutions of Hydrogen Peroxide $(30 \%$ VLSI grade Amplex Chemical Products Ltd) in deionized water [37]. At this dilution, the total etch time lasted 6 hours, during which various measurements (spectral and electrical) could be carried out at regular intervals of 2.4 minutes. The thickness calibration was performed by AFM measurements on a set of TFBGs etched for various durations and a linear fit of the results yielded a thickness in nm of $49-0.174 \times$ etch time in minutes.

2.4.4 Electrical measurements. The electrical measurements to determine the resistance of the silver film over grating region were performed as follows. The silver coated TFBG was fixed in the etch solution with metal clamps that also secured soft metal wires (each with a total resistance under $0.005 \Omega$ ) in contact with the coating on each side of the TFBG. The exact location of the TFBG was predetermined by launching red light in the fiber core and observing scattered light under a microscope. The metal wires were then connected to a multi-frequency LCR meter (Hewlett Packard HP 4274A) operated at 2 kHz. Since the electrical measurements were to be conducted in real time and in-situ, a measurement of the resistance of the etch solution without the silver coated-TFBG was made to ensure that there was no good electrical path other than the silver coating. The resistance of the highly dilute etch solution was larger than $12 \mathrm{M} \Omega$. The sheet resistance was approximated (because taken with 2-point probing only) by dividing the measured 
resistance by the distance between the probes and multiplying the result by the width of the coating, i.e. the $393 \mu \mathrm{m}$ cladding circumference. Resistance values were logged manually after each automatically captured optical spectrum. Because of this, the time interval between measurements was increased to 2.4 minutes, which caused an additional uncertainty of $+/-0.5 \mathrm{~nm}$ in thicknesses reported for the rate of etching reported in Section. 4.3 .

2.4.5. Simulation. Finally, simulations of the transmission spectra of silver coated TFBGs in the etch solution were carried out by first calculating the vector mode fields and effective indices of the core and cladding as a function of silver thickness and wavelength with a cylindrical finite-difference mode solver for layered structures with complex permittivity. Then, transmission spectra for P- and S-polarized input core guided light were obtained using complex coupled-mode theory to establish mode couplings at each wavelength and a Runge-Kutta algorithm to calculate the transfer function at each wavelength. The simulation tools used were our own, based on the algorithms reported in Ref. 38. The parameters used in the simulation of the fiber were: core radius $=4.1 \mu \mathrm{m}$, cladding radius $=62.5 \mu \mathrm{m}$, and core refractive index $=1.4535$, cladding refractive index $=1.4441$, silver coating thicknesses and refractive indices varied as in Fig. 2.10, external medium refractive index $=1.3181$ (based on the value of pure water at $1545 \mathrm{~nm}$ ) [33]. 


\subsection{References}

1. R. S. Sennett, G. D. Scott, "The structure of evaporated metal films and their optical properties," J. Opt. Soc. Am. 40 (4), 203-211 (1950).

2. M. S. Oldham, "Determination of optical constants in the near infrared of evaporated metal films from reflectance, transmittance and film thickness measurements," Ph. D Thesis, Iowa State University, America, 1949.

3. K. Ishiguro, G. Kuwahara, "Determination of the optical constants of Ag films from the measurements of intensity and phase change (I)," J. Phys. Soc. Japan 6 (2), 71-75 (1951).

4. V. M. Shalaev, Nonlinear Optics of Random Media, Springer, Berlin 2000.

5. A. S. Baburin, A. M. Merzlikin, A. V. Baryshev, I. A. Ryzhikov, Y. V. Panfilov, I. A. Rodionov, "Silver-based plasmonics: golden material platform and application challenges [Invited]," Opt. Mater. Express 9 (2), 611-642 (2019).

6. C. Percharroman, J. S. Moya, "Experimental evidence of a giant capacitance in insulator-conductor composites at the percolation threshold," Adv. Mater. 12 (4), 294$297(2000)$.

7. M. Breit, V. A. Podolskiy, S. Grésillon, G. V. Plessen, J. Feldmann, C. J. Rivoal, P. Gadenne, A. K. Sarychev, V. M. Shalaev, "Experimental observation of percolationenhanced nonlinear light scattering from semicontinuous metal films," Phys. Rev. B 64, 125106 (2001).

8. M. Hövel, B. Gompf, M. Dressel, "Dielectric properties of ultrathin metal films around the percolation threshold," Phys. Rev. B 81, 035402 (2010).

9. P. Gadenne, F. Brouers, V. M. Shalaev, A. K. Sarychev, "Giant stokes fields on semicontinuous metal films," J. Opt. Soc. Am. B 15 (1), 68-72 (1998). 
10. M. I. Stockman, D. J. Bergman, C. Anceau, S. Brasselet, J. Zyss, "Enhanced secondharmonic generation by metal surfaces with nanoscale roughness: nanoscale dephasing, depolarization, and correlation," Phys. Rev. Lett. 92, 057402 (2004).

11. E. Xenogiannopoulou, K. Iliopoulos, S. Couris, T. Karakouz, A. Vaskevich, T. Rubinstein, "Third-order nonlinear optical response of gold-island films," Adv. Funct. Mater. 18, 12811289 (2008).

12. S. D. Zuania, T. Peterseim, A. Berrier, B. Gompf, M. Dressel, "Second harmonic generation enhancement at the percolation threshold," Appl. Phys. Lett. 104, 241109 (2014).

13. S. M. Novikov, C. Frydendahl, J. Beermann, A. Zenin, N. Stenger, V. Coello, N. A. Mortensen, S. I. Bozhevolnyi, "White light generation and anisotropic damage in gold films near percolation threshold," ACS Photonics 4, 1207-1215 (2017).

14. H. Qian, Y. Xiao, Z. Liu, "Giant Kerr response of ultrathin gold films from quantum size effect," Nat. Commun. 7 (1), 13153 (2016).

15. J. Xu, J. Li, J. Huang, Z. Wang, W. Sheng, Y. Yang, R. Tan, G. Chen, X. Fang, W. Song, "An oxide-dispersed preparation strategy for silver ultrathin films with low percolation threshold thickness, subnanometer smoothness, and prominent durability," Adv. Mat. Interfaces 6, 1900608 (2019).

16. R. A. Maniyara, D. Rodrigo, R. Yu, J. Canet-Ferrer, D. S. Ghosh, R. Yongsunthon, D. E. Baker, A. Rezikyan, F. J. G. D. Abajo, V. Pruneri, "Tunable plasmons in ultrathin metal films," Nat. Photonics 13, 328-333 (2019). 
17. Y. Shan, G. Hu, M. L. Grilli, H. He, M. Zhu, Y. Zhao, J. Shao, "Measuring ultrathin metal coatings using SPR spectroscopic ellipsometry with a prism- dielectric-metalliquid configuration," Opt. Express 27 (6), 7912-7921 (2019).

18. R. Secondo, D. Fomra, N. Izyumskaya, V. Avrutin, J. N. Hilfiker, A. Martin, Ü. Özgür, N. Kinsey, "Reliable modeling of ultrathin alternative plasmonic materials using spectroscopic ellipsometry," Opt. Mater. Express 9 (2), 760-770 (2019).

19. W. J. Zhou, D. J. Mandia, M. B. E. Griffiths, S. T. Barry, J. Albert, "Effective permittivity of ultrathin chemical vapor deposited gold films on optical fibers at infrared wavelengths," J. Phys. Chem. C 118 (1), 670-678 (2014).

20. J. J. Tu, C. C. Homes, M. Strongin, "Optical properties of ultrathin films: evidence for a dielectric anomaly at the insulator-to-metal transition," Phys. Rev. Lett. 90 (1), 017402 (2003).

21. J. Albert, L. Y. Shao, C. Caucheteur, "Tilted fiber Bragg grating sensors," Laser \& Photonics Rev. 7 (1), 83-108 (2013).

22. Y. Shevchenko, C. Chen, M. A. Dakka, J. Albert, "Polarization-selective grating excitation of plasmons in cylindrical optical fibers," Opt. Lett. 35 (5), 637-639 (2010).

23. Y. Shevchenko, T. J. Francis, D. A. D. Blair, R. Walsh, M. C. DeRosa, J. Albert, "In situ biosensing with a surface plasmon resonance fiber grating aptasensor," Anal. Chem. 83, 7027-7034 (2011).

24. Y. Yuan, T. Guo, X. H. Qiu, J. H. Tang, Y. Y. Huang, L. Zhuang, S. G. Zhou, Z. H. Li, B. O. Guan, X. M. Zhang, J. Albert, "Electrochemical surface plasmon resonance fiberoptic sensor: in situ detection of electroactive biofilms," Anal. Chem. 88, 7609-7616 (2016). 
25. C. Caucheteur, T. Guo, F. Liu, B. O. Guan, J. Albert, "Ultrasensitive plasmonic sensing in air using optical fibre spectral combs," Nat. Commun. 7, 13371 (2016).

26. X. J. Zhang, S. S. Cai, F. Liu, H. Chen, P. G. Yan, Y. Yuan, T. Guo, J. Albert, "In situ determination of the complex permittivity of ultrathin $\mathrm{H}_{2}$-infused palladium coatings for plasmonic fiber optic sensors in the near infrared," J. Mater. Chem. C 6, 5161-5170 (2018).

27. J. J. Lao, P. Sun, F. Liu, X. J. Zhang, C. X. Zhao, W. J. Mai, T. Guo, G. Z. Xiao, J. Albert, "In situ plasmonic optical fiber detection of the state of charge of supercapacitors for renewable energy storage," Light Sci. Appl. 7, 34 (2018).

28. G. E. Villanueva, M. B. Jakubinek, B. Simard, C. J. Oton, J. Matres, L. Y. Shao, P. P. Millan, J. Albert, "Linear and nonlinear optical properties of carbon nanotube- coated single-mode optical fiber gratings," Opt. Lett. 36 (11), 2104-2016 (2011).

29. L. Y. Shao, M. B. Jakubinek, T. Sun, B. Simard, J. Albert, "Four-wave mixing in carbon nanotube-coated optical fiber gratins," Appl. Phys. Lett. 100, 071108 (2012).

30. F. Liu, J. Albert, "40 GHz-rate all-optical cross-modulation of core-guided near infrared light in single mode fiber by surface plasmons on gold-coated tilted fiber Bragg gratins," APL Photonics 4, 126104 (2019).

31. H. Raether, Surface plasmons: on smooth and rough surfaces and on gratings, Springer, Verlag 1988.

32. C. Caucheteur, C. Chen, V. Voisin, P. Berini, J. Albert, "A thin metal sheath lifts the EH to HE degeneracy in the cladding mode refractometric sensitivity of optical fiber sensors," Appl. Phys. Lett. 99, 041118 (2011). 
33. W. J. Zhou, Y. Zhou, J. Albert, "A true fiber optic refractometer," Laser Photonics Rev. 11 (1), 1600157 (2017).

34. R. Scherwitzl. S. Gariglio, M. Gabay, P. Zubko, M. Gilbert, J. M. Triscone, "Metalinsulator transition in ultrathin $\mathrm{LaNiO}_{3}$ films," Phys. Rev. Lett. 106, 246403 (2011).

35. J. R. Hendrickson, S. Vangala, C. Dass, R. Gibson, J. Goldsmith, K. Leedy, D. E. Walker, Jr, J. W. Cleary, W. Kim, J. Guo, "Coupling of Epsilon-Near-Zero mode to gap plasmon mode for flat-top wideband perfect light absorption," ACS Photonics 5, 776-781 (2018).

36. M. Z. Alam, J. Albert, "Selective excitation of radially and azimuthally polarized optical fiber cladding modes," J. Lightw. Technol. 31, 3167-3175 (2013).

37. X. J. Zhang, Z. Wu, F. Liu, Q. Q. Fu, X. Y. Chen, J. Xu, Z. C. Zhang, Y. Y. Huang, Y. Tang, T. Guo, J. Albert, "Hydrogen peroxide and glucose concentration measurement using optical fiber grating sensors with corrodible plasmonic nanocoatings," Biomed. Opt. Express 9 (4), 1735-1744 (2018).

38. Y. C. Lu, W. P. Huang, S. S. Jian, "Full vector complex coupled mode theory for tilted fiber gratings," Opt. Express 18 (2), 713-726 (2010). 


\title{
Chapter 3: Saturable absorption and bistable switching of single mode fiber core-guided light by a $6 \mathrm{~nm}$-thick, few layers graphene coating on the cladding surface
}

\begin{abstract}
The coupling of less than $80 \mu \mathrm{W}$ of in-plane polarized near-infrared light in a $6 \mathrm{~nm}$-thick graphene layer deposited on an optical fiber produces important permittivity changes leading to bistability and self-starting $50 \%$ modulation of over $1 \mathrm{~W}$ of continuous wave light in the core. These features arise from resonant coupling of core-guided light into the cladding by a 12-degree tilted, $1 \mathrm{~cm}$ long fiber Bragg grating (TFBG) via narrowband, polarization-dependent resonances that allow the selection of cladding modes with electric fields polarized in the plane of the graphene. The pulse repetition rate of the modulation increases from 10 to $269 \mathrm{~Hz}$ for input powers ranging from 0.3 to $1.33 \mathrm{~W}$ in the core, with no evidence of saturation. Investigations into the origin of these effects through physical modelling and different experimental conditions point to photo-induced Joule heating in the graphene layer giving rise to temperature increases of the order of $60^{\circ} \mathrm{C}$ and corresponding permittivity changes in the graphene and underlying silica fiber. Those changes lead to shifts in the resonance positions which result in the equivalent of saturable absorption for light guided in the core without direct contact with the absorbing graphene layer.
\end{abstract}

\subsection{Introduction}

Graphene has many interesting applications in several areas of physics, electronics and photonics, including an ultrafast carrier mobility and a high intrinsic thermal conductivity along the plane of its two-dimensional structure [1-5], including thermal bistability [6-10]. 
While switching based on thermal bistability is a relatively slow process, it still may be used for applications where ultrafast response speed is not necessary such as optical routing and switching [7-8,10-11]. In fact, several silicon-based thermally bistable photonic integrated devices using graphene-based flexible heat conductors have been demonstrated, such as waveguide resonators [6], microfiber phase shifters [7,8], photonic ring resonators [9], and micro gratings [10]. In contrast with the various configurations used so far, a very simple, monolithic, and low insertion loss all-fiber structure is proposed and demonstrated here, comprising a tilted fiber Bragg grating (TFBG) inscribed in a standard telecom singlemode fiber with a nanometer scale graphene coating on the surface of the cladding of the fiber. This configuration does not require to physically modify the fiber by etching, tapering or side polishing $[7-8,10]$. Furthermore, and this is a very unique property of the proposed configuration: the graphene layer is never in contact with the core-guided light and a very small fraction (less than $0.01 \%$ ) of the input light is actually needed to modulate it by more than $50 \%$. The demonstration of the ability to excite novel nanomaterial layers with controlled amounts of light intensity and polarization on the surface of un-modified standard optical fibers represents a significant advance in materials research. One straightforward application of the configuration shown here is Q-switching of fiber lasers, with the particular advantage that all of the laser light would not go through the graphene layer but only a very small portion of it, and also that the fiber would not have to be physically modified $[12,13]$. Fibers used here have inherently zero transmission loss for the lengths used in such experiments, near zero insertion loss, and a wide array of relatively low-cost fiber-coupled instrumentation (sources, detectors, spectrum analysers, 
polarization controllers) is available because of the telecommunication industry and associated R\&D activities.

A TFBG written in the core of a fiber by an ultraviolet light induced photosensitive process enhances the coupling of light from the single forward-propagating core mode to a large number of backward propagating cladding modes. Coupling to each cladding mode occurs at different wavelengths to satisfy the phase matching relation between the effective index of the core, the effective index of one of the cladding modes and the grating period. This results in a low insertion loss transmission spectrum that includes a large number of discrete lossy resonances with a Q-factor greater than $10^{4}$, separated by $0.2-2 \mathrm{~nm}$ and distributed over a broad spectral range (10-100 nm depending on tilt angle) [14]. These cladding modes interact with the external medium through their evanescent fields and thus penetrate the graphene layer where they are partially absorbed, generating heat. An important feature of TFBGs is that the evanescent field of the cladding modes can be oriented either parallel or perpendicular to the cladding surface by linearly polarizing the input core light in S- or P-polarization relative to the tilt plane [14]. Upon any change in the external medium permittivity, the narrow cladding resonances shift in wavelength and/or amplitude, resulting in large transmission level modifications within their spectral width. This high sensitivity to very small changes in the TFBG surrounding has been used previously to develop high performance sensors for biomedicine $[15,16]$, electrochemical and magnetic detection [17,18], gas monitoring [19,20], and renewable energy storage facilities [21] Here, permittivity changes are due to evanescent field absorption by graphene to demonstrate a platform for light-matter interaction with a simplified waveguiding structure, very low intrinsic loss, and ease of fabrication [22-24]. 
In chapter 3, the optical bistability and self-pulsing properties of graphene-coated tilted fiber Bragg gratings (G-TFBG) are studied experimentally as a function of the core propagating input light power, polarization and wavelength. Modulation amplitudes larger than $50 \%$ and pulse repetition rates up to $269 \mathrm{~Hz}$ are obtained for input light powers near $1 \mathrm{~W}$ in the core of the fiber. With regards to the polarization dependence however, significant modulation amplitudes were only obtained for light polarized tangentially (along the graphene plane), in spite of the preliminary results for broadband pumping that showed little polarization sensitivity. These results are discussed in the context of a bistable scheme based on nonlinear positive feedback of the output power on the system transmission [25-29].

\subsection{Photo-thermal effects in graphene coated-TFBG}

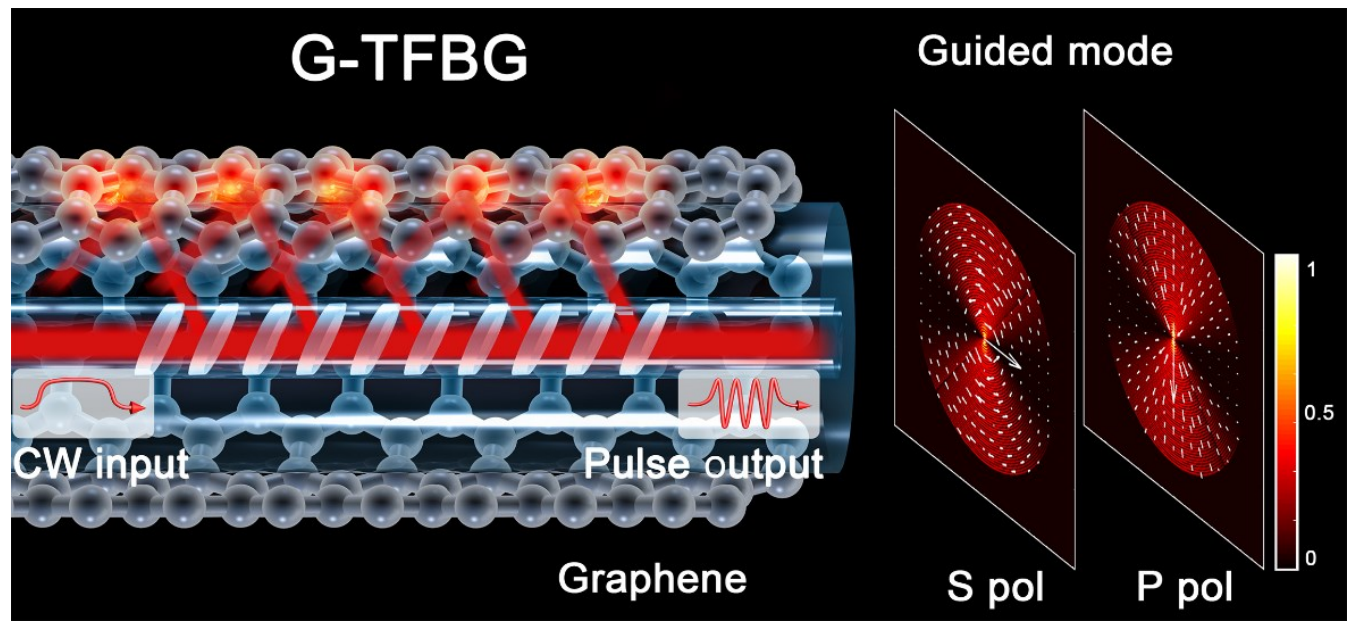

Fig. 3.1. Schematic diagram of the G-TFBG. The CW laser input power is coupled out of the core by the cladding mode resonance that in turn modulates the graphene and fiber permittivity by photoinduced Joule heating. The transmission of the $\mathrm{CW}$ laser at the pumping wavelength gets modulated as the grating resonances shift in response to temperature and refractive index change of graphene. Intensity (color bar) and orientation of the electric field of radially and azimuthally polarized cladding modes are also shown. 
The measured transmission spectrum of a TFBG contains a multitude of narrowband resonances corresponding to contra-directional coupling from the single core mode to the many available modes guided by the much larger fiber cladding. The wavelengths of the cladding mode resonances $\left(\lambda_{\text {clad }}\right)$ depend on the phase matching provided by the grating period $(\Lambda)$ between the propagation constants of the core, $N_{\text {eff }}($ core $)$, and that of the coupled cladding mode $\mathrm{N}_{\text {eff }}($ clad $)$, as $\lambda_{\text {clad }}=\left(\mathrm{N}_{\text {eff }}(\right.$ core $)+\mathrm{N}_{\text {eff }}($ clad $\left.)\right) \Lambda$. As a result, if the effective index of a cladding mode is modified, the corresponding resonance shifts. While the core and the cladding of the fiber are extremely transparent at the wavelengths used (in the telecommunication band around $1550 \mathrm{~nm}$ ), graphene on the cladding surface can absorb some of the cladding mode power, leading to permittivity changes and heat. This effect is proportional to the fraction of cladding mode power propagating the very thin graphene layer, where the temperature increase is essentially instantaneous because of the small volume involved. Heat then diffuses with some delay to the underlying glass and changes its refractive index through the photothermal effect. For the glass, the only consequence is a change in the real part of the refractive index, but for graphene both the real and imaginary parts can be modified by the absorped power. Depending on the power density present in the graphene, its properties may depend nonlinearly on the absorbed light power. All these interactions are also polarization-dependent as the conductivity of very thin graphene (as that of all "2D" materials) is very different for in-plane and out-of-plane electric fields of the pump light, a property which can be selected by a TFBG [30]. When a broadband input pump light source is used, i.e. much broader than individual resonances, even if the resonance wavelengths shift under optical pumping, this does not cause a significant change in the amount of power coupled from the core to the cladding and then to the 
graphene material, yielding a stable, but different, G-TFBG transmission spectrum. In resonant pumping on the other hand, a laser with a linewidth much narrower than the spectral width of the cladding mode resonances is used. In this case, any shift of the cladding mode resonance results in an immediate change in the coupling efficiency and hence to the pumping level into the graphene layer, which in turn modifies the heat generation rate. This feedback system leads to time-dependent fluctuations of the system transmission. The dynamics of these fluctuations depend strongly on the initial position of the laser wavelength within a resonance spectral width and on the pump power. As will be demonstrated below, resonant pumping can result in self-starting oscillations of the transmitted power from the pump laser. The frequency of the oscillations depends on the input power and their amplitudes depend on the initial detuning of the pump from the resonance.

The current manuscript aims at studying the effect of broadband and resonant pumping on the static and dynamic power transmission of the G-TFBG, as a function of input power, cladding mode polarization, and, in the case of resonant pumping, the wavelength of the laser in relation to its position in the spectral band of a resonance.

\subsection{Materials and methods}

The graphene film was synthesized on a copper surface by atmospheric pressure chemical vapor deposition (APCVD) with acetylene $\left(\mathrm{C}_{2} \mathrm{H}_{2}\right)$ as the carbon precursor. This process can dramatically decrease the defects in synthesized graphene. A five-layer graphene film can be obtained by synthesis at a temperature of $850^{\circ} \mathrm{C}$ for 60 minutes [31,32]. After the synthesis, the copper foil supporting the deposited graphene was etched by using an $\left(\mathrm{NH}_{4}\right)_{2} \mathrm{~S}_{2} \mathrm{O}_{8}$ solution and rinsed in deionized water several times. 
The $12^{\circ}$ TFBG $(1 \mathrm{~cm}$ length) was fabricated in photosensitive single mode fiber (CORNING SMF-28) using $248 \mathrm{~nm}$ light from a pulsed high-energy KrF excimer laser (model PM-848 from Light Machinery Inc.) operated at $248 \mathrm{~nm}$ and $40 \mathrm{~mJ}$ per $20 \mathrm{~ns}$ pulse at $100 \mathrm{pulses} / \mathrm{s}$ with the phase mask technique. Optical transmission spectra were measured with an Erbium amplified emission source (ASE) and an optical spectrum analyzer (ANDO AQ 6317B) with a resolution of $0.02 \mathrm{~nm}$. A polarization controller (OZ OPTICS. LTD.) was used to linearize the input light polarization either in the tilt plane of the TFBG (Ppolarized) or out of plane (S-polarized), as determined by maximizing either the short or long wavelength resonance of the polarization-dependent pairs of resonances (Fig. 3.2(a)) [30]. As discussed in Refs 14 and 30, the polarization convention used here refers the orientation of linearly polarized core guided input light relative to the plane in which the grating fringes are tilted. Furthermore, an important feature of TFBGs is that high order cladding modes excited by P-polarized light are polarized almost $100 \%$ in the radial direction (i.e. perpendicular to the cladding surface) while those excited by S-polarized input light are polarized azimuthally (i.e. parallel to the cladding surface). In the remainder of this chapter the spectrum resonances corresponding to those two types of excitation will be referred as "P-polarized" and "S-polarized" resonances.

The graphene film was transferred onto the surface of TFBG by contacting the side of the fiber with the edge of the graphene sheet and wrapping it in a single turn around the fiber circumference. Fig. 3.2(b) shows the effect of the graphene coating on the transmission spectrum of the TFBG: mostly a small attenuation and broadening of the $\mathrm{S}$ and $\mathrm{P}$ resonances. Given that the $\mathrm{S}$ resonances are deeper by about $2 \mathrm{~dB}$ than the $\mathrm{P}$ ones in the bare TFBG and end up several $\mathrm{dB}$ shallower in the graphene coated TFBG, it is obvious 
that the graphene impacts S polarized modes (electrical field of the mode in the plane of the graphene) much more than P polarized ones. Furthermore, the significantly decreased spacing (from $240 \mathrm{pm}$ to $72 \mathrm{pm}$ ) between resonances in the coated sample relative to the bare one shows that the real part of the mode effective indices of S and P modes are also modified differently. The induced loss following the addition of the very thin graphene layer must be attributed to mechanisms that include absorption by graphene but likely also some scattering. While the spectral changes just noted are certain, the exact resonance positions and amplitudes cannot be compared between Fig. 3.2(a) and 3.2(b) as they were measured on different (but similar) gratings.
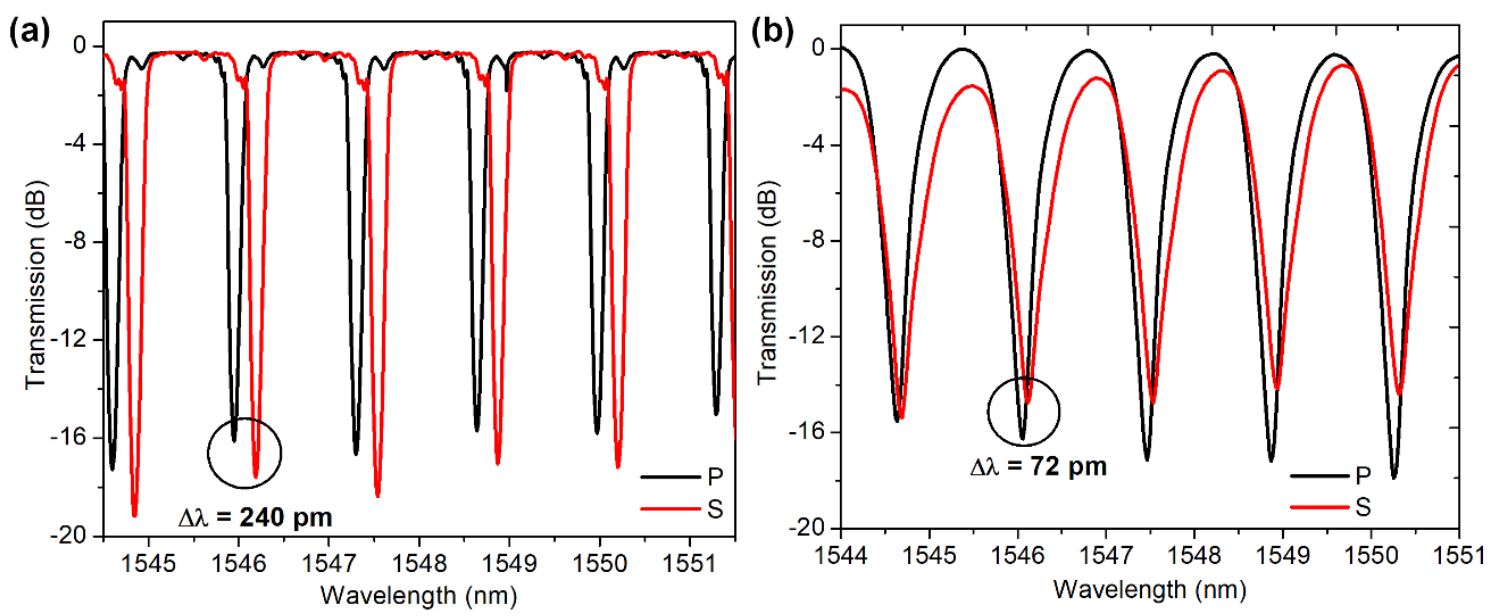

Fig. 3.2. Transmission spectra of (a) bare; and (b) graphene-coated TFBG (1 cm-long, 12 degree tilted), both in air. 

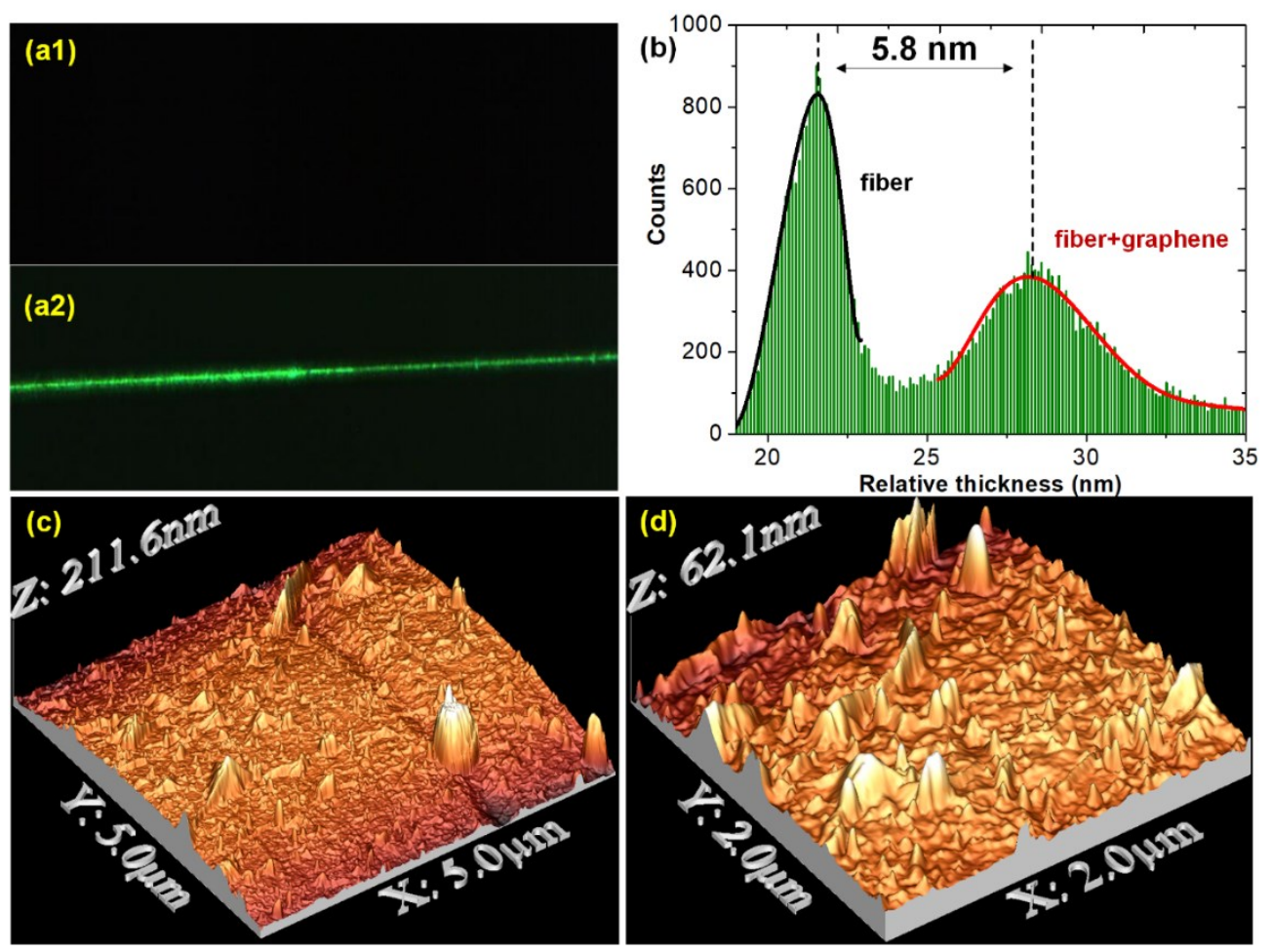

Fig. 3.3. Microscope images of $12^{\circ}$ TFBG with $514 \mathrm{~nm}$ green light coupled into the core (a1) without graphene on the cladding, and (a2) with graphene; (b) average thickness of graphene from AFM height histograms; AFM images over (c) $5 \mu \mathrm{m} \times 5 \mu \mathrm{m}$, and (d) $2 \mu \mathrm{m} \times 2 \mu \mathrm{m}$.

For the loss, a contribution from scattering is supported by the fact that when visible light is coupled into the core, a significant amount of light can be observed to come out of the grating region resulting from the fact that the input light at $514 \mathrm{~nm}$ is experiencing a small grating coupling to cladding mode through a third order (fundamental cladding mode resonance is around $1550 \mathrm{~nm}$ ) phase matching equation, but only when graphene is present (Fig. 3.3(a)). Indeed, an examination of the graphene surface by atomic force microscopy (AFM) reveals a relatively rough surface with an average thickness of $5.8 \mathrm{~nm}$ with random variations of the order of $+/-3 \mathrm{~nm}$ (Fig. 3.3(b)-3(d)).

Light-induced variations of the G-TFBG static and dynamic transmission characteristics were carried out as follows. For broadband pumping, the output of an 
Erbium-doped fiber amplifier (Amonics AEDFA-33-B-FA) was coupled in the core of the fiber without input seed laser signal. The total broadband power was controlled by the driving current of the amplifier pump laser. On the other hand, for resonant pumping the narrowband light from a tunable laser source (HP 81640A tunable laser, power: $2 \mathrm{~mW}$ ) was amplified by the same amplifier before being coupled into the fiber. In both cases, the polarization controller was set to either S- or P-polarization of the input light by an initial spectrum measurement with the amplifier without seed and at low power. In the resonant case, the output spectrum is unstable and cannot be measured; dynamic (time-dependent) transmitted power measurements of the narrowband pump source were then carried out with a photo-detector (New Focus D400FC) and monitored with an oscilloscope (Rigol DS4054 Digital Oscilloscope).

\subsection{Results}

\subsubsection{Non-resonant pumping}

From the characterizations shown in Fig. 3.4, it is clear that the loss induced by graphene is at least partly due to scattering. But linear absorption also plays a role in the spectrum modifications due to the graphene layer, since this material has a complex refractive index at these wavelengths (2.52-2.24i) [7]. Separating the contributions of scattering and absorption can be carried out by monitoring resonance wavelength shifts amplitude changes as a function of input power. Scattering does not lead to refractive index changes and therefore to wavelength shifts, while absorbed power does because of the heat generated which leads to refractive index changes. With regards to resonance amplitude changes, they can come about from two sources: 1) absorption changes in the graphene layer; 2) effective index chirp along the grating (which widens and flattens the resonances). 
It is more difficult to distinguish between these two causes of resonance amplitude changes but chirp typically induces asymmetry in the spectral shape of resonances while absorption causes a symmetrical widening.
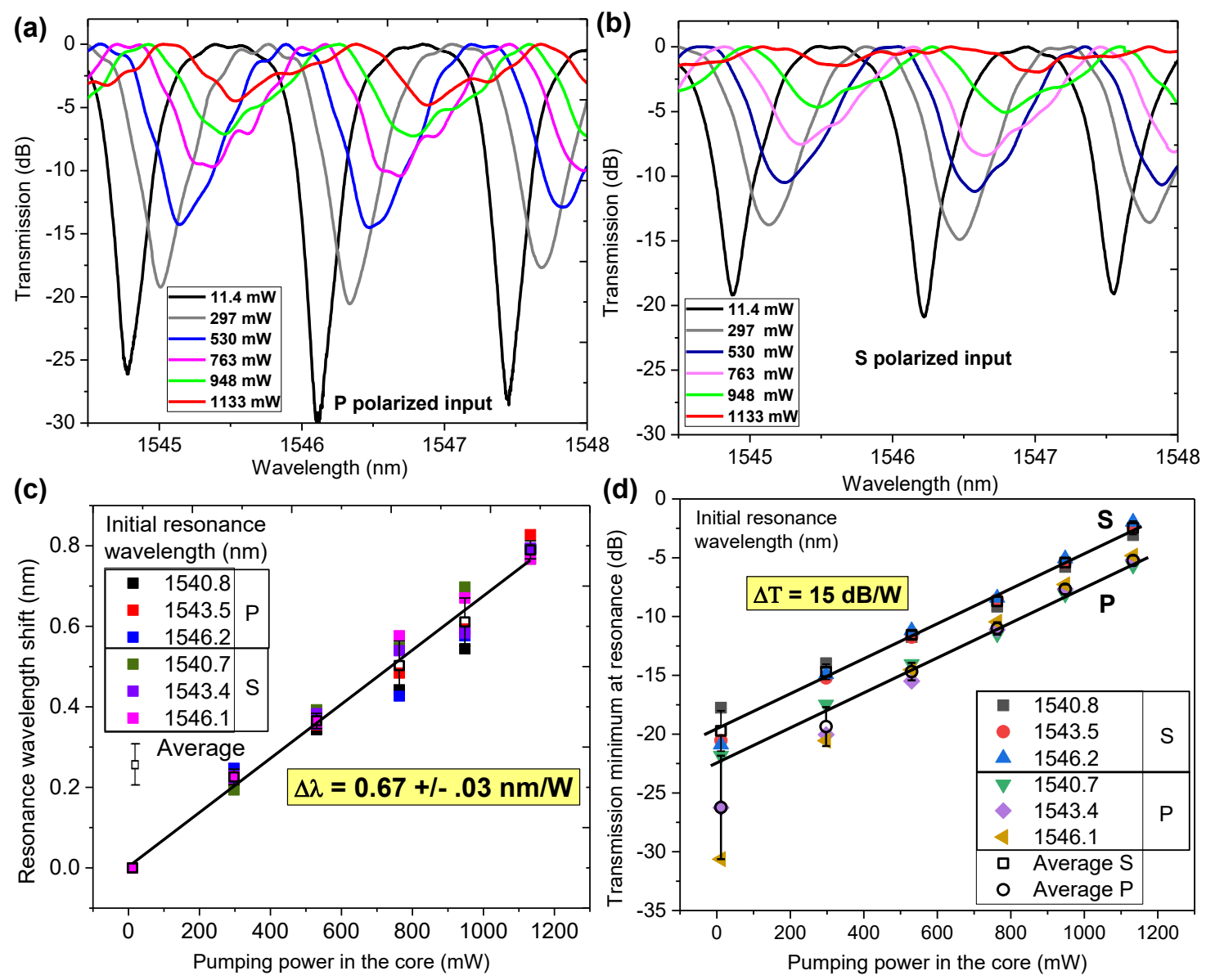

Fig. 3.4. Measured normalized transmission of G-TFBG for several values of input power, and two input core mode polarization: a) P-polarized (radial cladding mode electric field at fiber surface); b) S-polarized (tangential electric field at fiber surface). Note: The actual un-normalized spectra are offset vertically by the change in input power: here, the highest value of the transmitted power was used as the normalization level. Evolution of several S and P resonance wavelengths (c) and amplitudes (d) as a function of input power in the core mode. 
Fig. 3.4(a) and 3.4(b) shows the effect of broadband pumping with S- and P-polarized light on the transmission spectrum of G-TFBG. As expected, in all cases the resonances red-shift, broaden (while becoming somewhat asymmetric, indicating potential optical bistability [33-35]) and attenuate with increasing pump power and these effects are stable in time. With regards to the origin of the optical bistability in graphene, the thermo-optical nonlinearity is likely dominant in our case while the free-carrier dispersion and optical Kerr effect can be neglected due to the relatively weak broadband pumping power and the millisecond scale responses to be shown in section 3.4.2 [36-38]. In order to extract information from the modified spectra, the wavelength and the minimum transmission of typical S and P resonances are plotted in Fig. 3.4(c) and 3.4(d) as a function of the total input power in the fiber core. For wavelength, an average linear shift of $670 \mathrm{pm} / \mathrm{W}$ of input power in the core is obtained $(+/-26 \mathrm{pm} / \mathrm{W})$, regardless of polarization, for the six resonances analyzed. The net coupling strength (as indicated by the amplitude of the resonance in transmission) also changes quite linearly (especially at higher powers and transmission levels). The slope of the amplitude changes with power is the same for $\mathrm{S}$ and P polarized input, and they remain separate. 

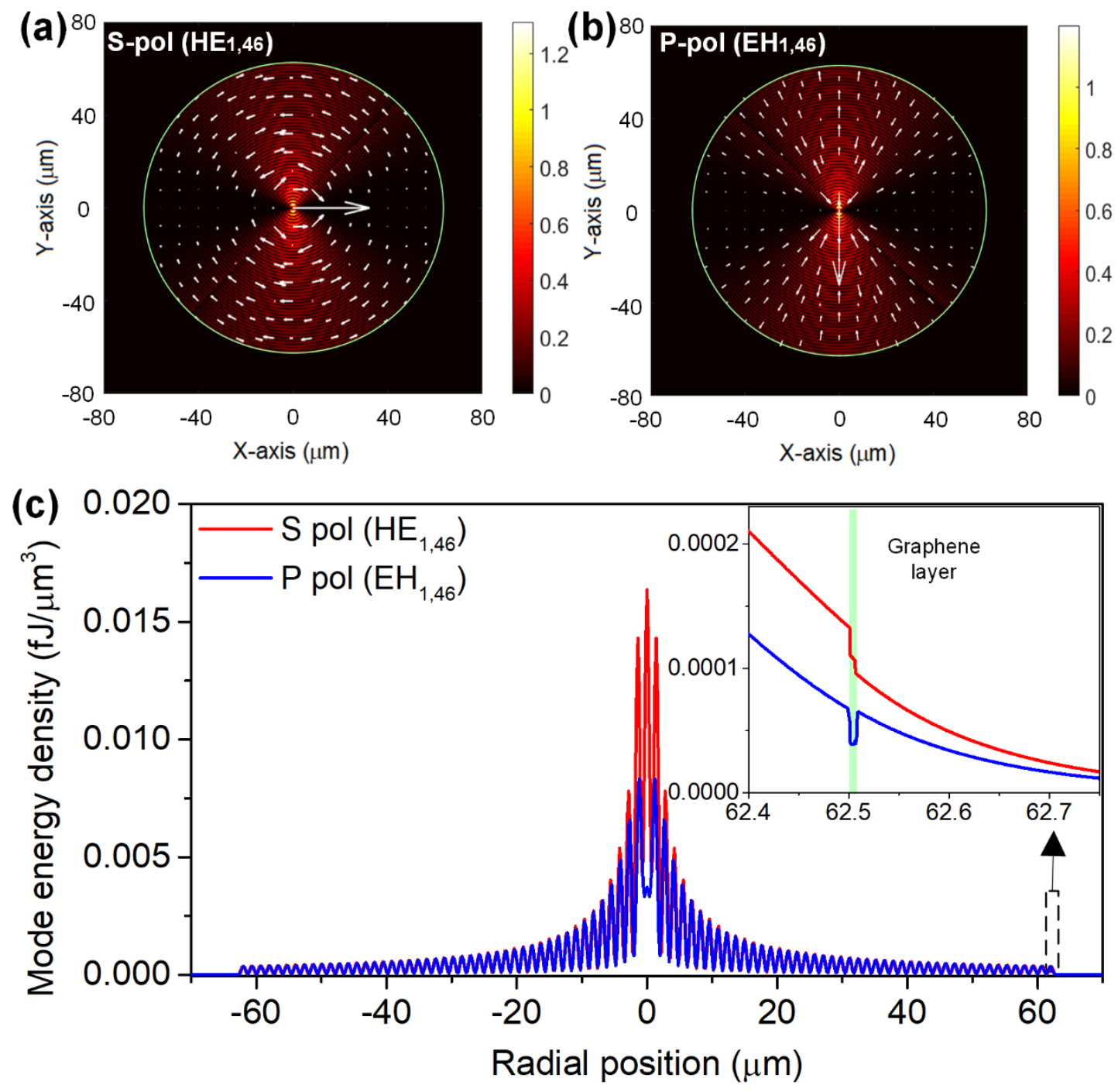

Fig. 3.5. Simulation of the electric field of a mode of the graphene coated cladding for (a) Spolarization mode (E field tangent to fiber surface); (b) P-polarization mode (E field radially oriented); (c) mode energy density distribution of the two modes along the fiber diameter. The inset shows the the energy distribution in and around the graphene layer. The overall shapes of the modes in (a) and (b) are essentially identical for the uncoated fiber, while the discontinuity at the cladding surface in the inset of (c) is slightly different but not relevant to the results and concepts presented.

In order to understand these results, we first compute an estimate of the amount of optical power absorbed by the graphene layer. Fig. 3.5 shows the simulation of the electric 
field of a graphene-coated fiber at the resonance pumping position ( $\mathrm{HE}_{1,46}(\mathrm{~S}$ mode) and $\mathrm{EH}_{1,46}$ (P mode)). The modes are calculated with a complex finite-difference vectoral simulation tool (FIMMWAVE, by Photon Design). The following parameters are used: 8.2 $\mu \mathrm{m}$ core diameter with refractive index 1.449311, $125 \mu \mathrm{m}$ cladding diameter with refractive index 1.444078, graphene thickness and complex refractive index as noted at the beginning of this section, and surrounding medium of air (refractive index=1.00027). For the modes corresponding to resonances near $1546 \mathrm{~nm}$ (i.e. those used in our experiments), the effective indices found by phase matching from the grating period are close to 1.33 , corresponding to radial mode order 46 in the mode list found by simulation. The inset of Fig. 3.5(c) shows how the mode energy is distributed in and around the graphene layer, with discontinuities reflecting the different boundary conditions for the fields of the modes and the relatively large permittivity differences occurring at the graphene boundaries. The fraction of the mode energy density located in the graphene layer is $7.4 \times 10^{-5}$ for the $\mathrm{S}$ mode and $4.4 \times 10^{-5}$ for the P mode, and these values do not change much over the spectral range where this TFBG has significant resonances. Therefore, for a given input power and similar integrated core to cladding coupling for $\mathrm{S}$ and $\mathrm{P}$ modes, there would be almost twice as much energy dissipated in the graphene for $\mathrm{S}$ modes than for $\mathrm{P}$ modes, so the rate of heating should be significantly higher. However, Fig. 3.4 shows that the power coupling from the core to the cladding is higher for $\mathrm{P}$ resonances than for $\mathrm{S}$ resonances. The fact that the wavelength shifts for both polarizations are nearly the same reveals that the difference in energy density in the graphene is compensated by the difference in coupling efficiencies into cladding modes. More importantly however, under continuous broadband pumping, energy keeps being dumped into graphene at a very high rate regardless of where the 
resonances shift or their exact amplitudes, as long as there is some coupling (i.e. the coreto-cladding mode resonances do not flatten out completely). In this situation it is reasonable to expect the TFBG-graphene system to converge to a steady state that does not really depends on polarization but rather on the heat dissipation properties of the graphene surroundings. A difference due to the heating rate would appear only briefly upon the start of the pumping, on a time scale of the order of ms and before the whole system is driven towards thermal equilibrium between heat generation (absorbed light power) and heat dissipation. This dissipation occurs mostly via conduction in the graphene and the glass, and possibly by convection in the surrounding air. Since the dissipation rates are proportional to the temperature difference $\Delta \mathrm{T}$ between the heated body (the graphene) and its surroundings, and heat generation is proportional to the absorbed power, equilibrium will be reached when:

$$
\mathrm{A} \times \Delta \mathrm{P}=\mathrm{C} \times \Delta \mathrm{T}
$$

where $\Delta \mathrm{P}$ is the amount of power absorbed by the graphene while $\mathrm{A}$ and $\mathrm{C}$ are constants related to geometry and to the thermal properties of the materials in the system. The amount of power absorbed is further related to the device parameters by:

$$
\Delta \mathrm{P}=\mathrm{P}_{\text {in }} \times \mathrm{F}_{\text {clad }} \times \mathrm{P}_{\text {frac }} \times \text { a effective }
$$

where $\mathrm{P}_{\text {in }}$ is the input power in the core, $\mathrm{F}_{\text {clad }}$ the fraction of the core guided light coupled to the cladding mode, $P_{\text {frac }}$ the fraction of cladding mode energy in the graphene layer, and aeffective an effective absorption coefficient for the graphene layer located on the TFBG. Therefore, there is a one-to-one relationship between the input power in the core and the steady-state temperature of the system, and the experiments show that it is the same for S 
and P polarized inputs. Finally, in fiber gratings the wavelength shifts of resonances can be related to the thermal coefficient of the silica glass ( $\mathrm{dn} / \mathrm{dT}$ near $\left.10^{-5} / \mathrm{degree}\right)$ as follows: first, the phase matching condition can be used to derive the following relationship between wavelength shifts and input power:

$$
\Delta \lambda / \Delta \mathrm{P}=\Delta\left(\mathrm{N}_{\text {eff }}(\text { core })+\mathrm{N}_{\text {eff }}(\text { clad })\right) \times \Lambda / \Delta \mathrm{P}
$$

(where $\Lambda$ is the grating period along the fiber axis, equal to $557.2 \mathrm{~nm}$, and $\mathrm{N}_{\text {eff }}$ the effective index of the core and cladding mode being coupled). We can replace $\Delta\left(\mathrm{N}_{\text {eff }}(\right.$ core $\left.)+\mathrm{N}_{\text {eff }}(\mathrm{clad})\right)$ by $(2 \times \mathrm{dn} / \mathrm{dT}) \times \Delta \mathrm{T}$ since the effective index changes are entirely due to the temperature increase and almost perfectly equal for core and cladding modes [14]. Therefore, the average temperature increase of the graphene layer at equilibrium as a function of input power can be found as:

$$
\Delta \mathrm{T} / \Delta \mathrm{P}=(\Delta \lambda / \Delta \mathrm{P}) /(2 \times \mathrm{Lg} \times \mathrm{dn} / \mathrm{dT})=60^{\circ} \mathrm{C} \text { per } \mathrm{W} \text { of input per power }
$$

With regards to the amplitude dependence on pump power, it can also be related to temperature increases because the cladding mode power distribution along the grating length is highly non-uniform: from coupled mode theory for contra-directional coupling, it follows a $\sinh (C(z-L g))^{2}$ dependence (where $z$ is the axial position along the grating, $C$ is a coupling constant and $L g$ the length of the grating) (i.e. an exponential-like decay from the beginning of the grating to the end) [39]. The grating is much hotter at its input than at its end. Therefore, the effective index shifts are also non uniform and phase matching occurs at different wavelengths along the grating, which is equivalent to a period chirp. The grating response is then weaker at each wavelength and distributed over a broader wavelength range around the original resonance, as seen on the experimental results in Fig. 
3.4(a) and 3.4(b). This mechanism of a purely thermal effect explains the fact that the slopes of the amplitude changes in Fig. 3.4(d) are independent of polarization, as were the wavelength shifts. The constant separation between the S and P results comes solely from the starting point, as the $\mathrm{P}$ resonance is stronger at zero pumping because it was less attenuated by the addition of graphene on the cladding (Fig. 3.2).

\subsubsection{Self-starting modulated switching under resonant pumping}

A second group of experiments was carried out by resonant pumping (See Fig. 3.9 in the Supporting information for the experimental set-up). First, an amplified, S-polarized laser signal was launched in the core of the G-TFBG at different wavelength positions relative to a cladding mode resonance. The relative positions of the laser wavelength and transmission resonance was verified by a spectrum measurement obtained with low laser light power and simultaneous low power broadband light in the device. Then, the output was switched to the power detector instead of the spectrum analyzer, and the timedependent transmission measured as a function of the laser wavelength, for a fixed value of the amplified laser power. The results shown in Fig. 3.6 indicate that for S-polarized input (E-field mode polarization in the plane of the graphene) a strong modulation of the output power occurs for several laser wavelength positions.

While the average output power levels in Fig. 3.6(b) change in accordance with the transmission factor of the G-TFBG for the tested wavelengths, as shown in Fig. 3.6(a), the strongest modulation of $56 \%$ of the average transmitted power occurs when the pump signal wavelength is near the middle of the resonance upward slope on the long wavelength side. Negligible modulation occurs for pumping on the short wavelength side of the resonance, or for pumping at any wavelength when using P-polarized input (Fig. 3.6(c)). 

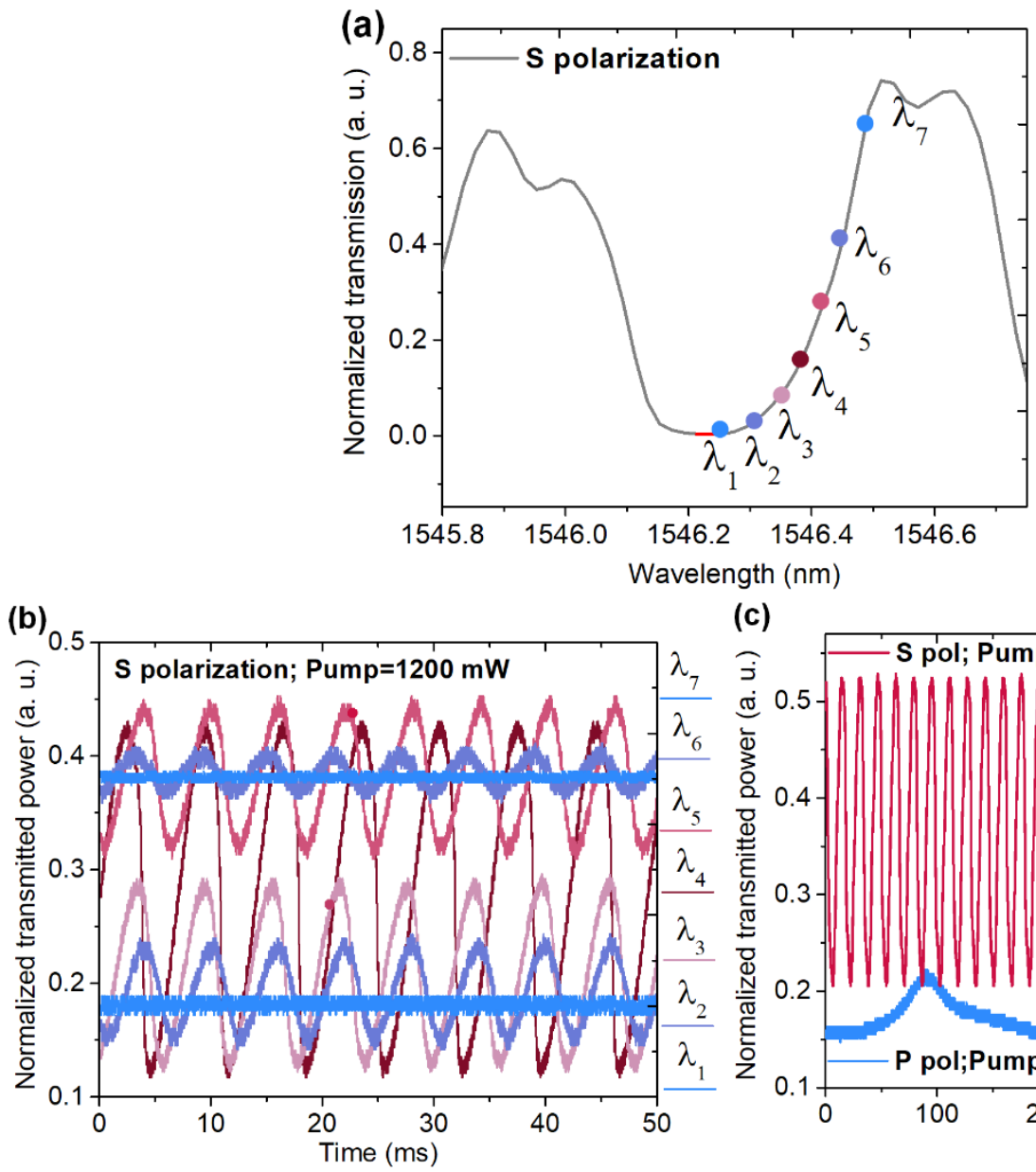

(c)

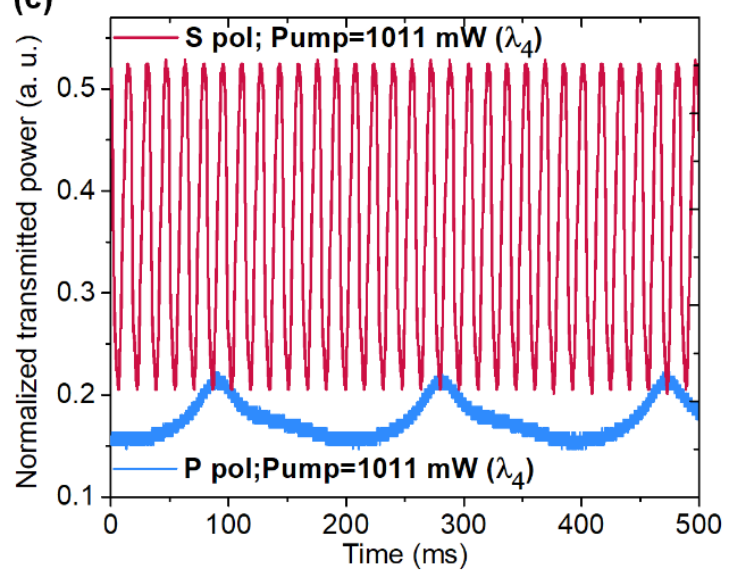

Fig. 3.6. (a) $\mathrm{CW}$ pumping wavelengths used relative to the resonance spectrum (transmission converted from $\mathrm{dB}$ to linear units for better correspondence with parts (b-d)); (b) Self-pulsing response for wavelengths shown in (a); (c) Input polarization dependence.

This general behavior can be explained as a dynamic bistability of the transmitted light caused by the feedback of the amount of power transmitted on changes of the position of the resonance due to heat from absorbed cladding mode power. In simple terms, power coupled to a cladding mode by a pump on the long wavelength side heats up the grating and causes a red shift of the resonance, as seen in the broadband pumping case. In single wavelength pumping however, this shift first increases the coupling of power to the cladding (because the maximum coupling wavelength of the resonance becomes closer to 
the pump wavelength), which further accelerates the red shift of the resonance wavelength, which increases the heating rate further. This continues to accelerate until the resonance wavelength becomes equal to the pump wavelength. As the graphene continues to heat up however, the resonance wavelength red shifts past the pump wavelength and the coupling to cladding modes starts to decrease, thereby decreasing the rate of heating. Then the fiber eventually cools down by the same dissipative mechanisms seen in broadband pumping. As a result, the resonance begins to shift back towards shorter wavelengths (and higher coupling to cladding modes for higher heating rate) for the cycle to restart. In order to investigate this further, another experiment was carried out by fixing the pump wavelength at $\lambda_{4}$ (where the maximum modulation is observed) but varying the input power. Results from Fig. 3.7 show that the frequency (or pulse repetition rate) of the self-starting oscillations increases with input power, reaching $269 \mathrm{~Hz}$ at the maximum power that could be tested with our equipment, and without loss in the amplitude of the modulation. 


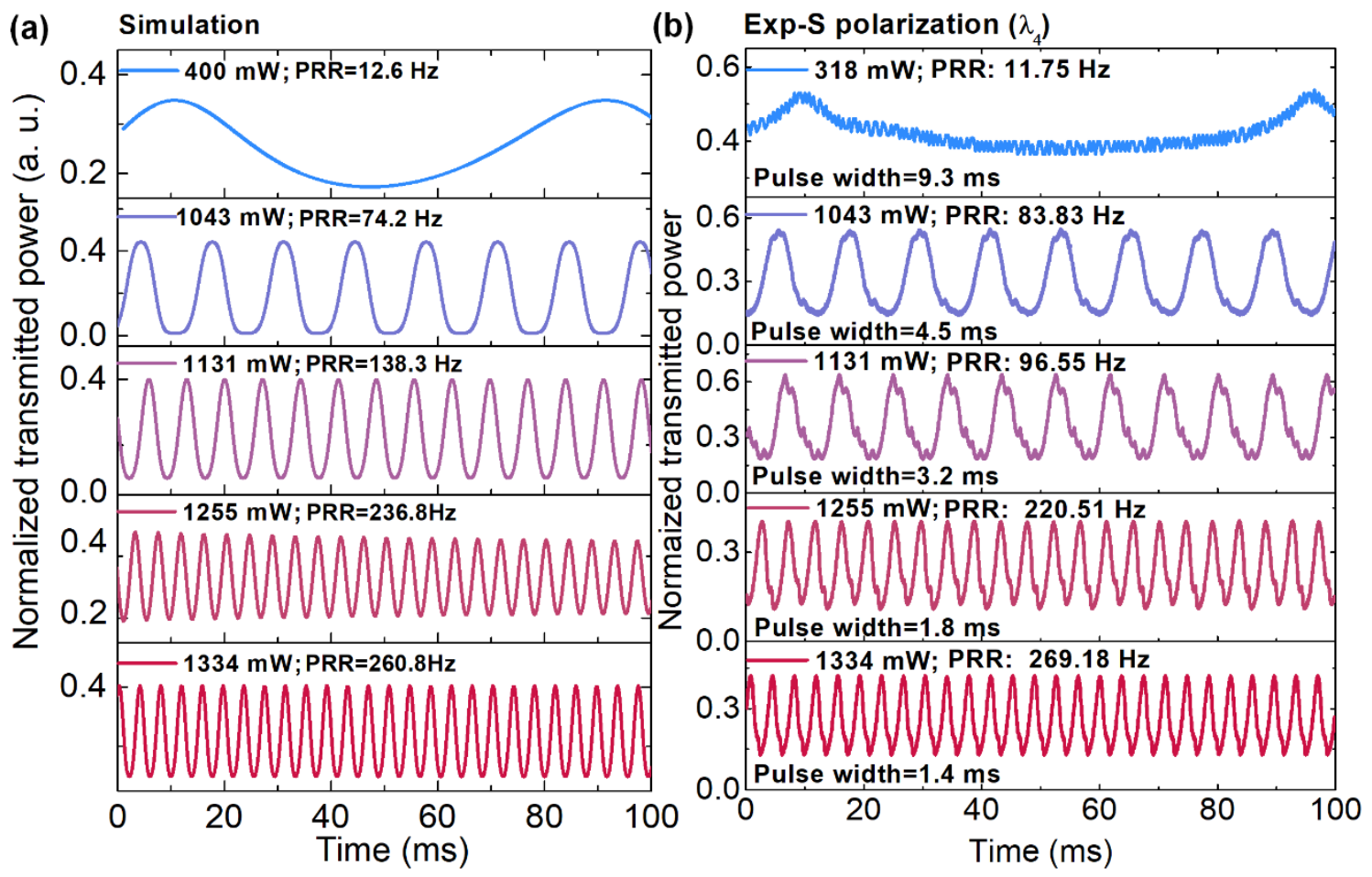

(b) Exp-S polarization $\left(\lambda_{4}\right)$

Fig. 3.7. Self-pulsing response to pump power: a) simulation; b) measured.

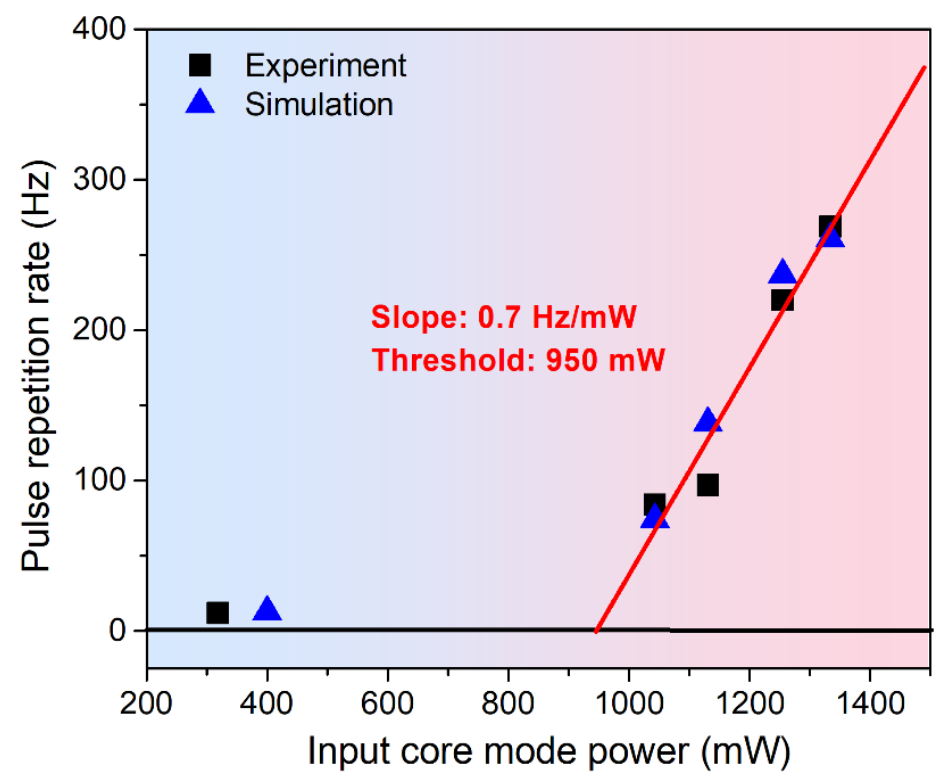

Fig. 3.8. The self-pulsing repetition rate as a function of pump power in the core. 
The trend in frequency vs input power (Fig. 3.8) shows an apparent threshold near $950 \mathrm{~mW}$ and a non-saturating increase of $0.7 \mathrm{~Hz} / \mathrm{mW}$. It is expected for the modulation rate to increase with input power because this increases the heating rate as well as the cooling rate (as defined in Equation 3.4), according to our simple model. This model for self-pulsing is based on a simplified one-dimensional finite-difference simulation of the thermal diffusion equation for the temperature on a $125 \mu \mathrm{m}$ long spatial grid for the radial position " $r$ " oriented along a diameter of the coated fiber, with time-dependent heat sources at the fiber surface locations. The heat sources were calculated from absorption of the cladding mode power according to Equation 3.2. The initial state of the system was fixed at room temperature everywhere and an initial heat input was calculated from the power coupling and absorption in graphene at the initial value of the resonance position. Then at each time step the temperatures were updated across the simulation domain, with a time step $\Delta \mathrm{t}=\Delta \mathrm{r}^{2} / 2 \alpha$ to ensure numerical stability, where $\Delta \mathrm{r}=1 \mu \mathrm{m}$ is the spatial discretization and $\alpha$ is a power dependent thermal diffusivity parameter determined empirically. The updated temperatures were used to recalculate the resonance shift and to find new values of the absorbed power for the next time step (see Supplementary note 2 in the Supporting information for further details). Results shown in Fig. 3.7(a) indicate that this model can reproduce the power dependent increase of the self-pulsing frequency, provided that the thermal diffusivity parameter changes with input power. This relationship is believed to originate at least partly in changes of the graphene absorption and permittivity as a function of the power propagating in it. Note that the simulation results in Fig. 3.7(a), show the steady state oscillations that begin a few ms after the input power is introduced into the system. 
In fact, a better estimate of the efficiency of power dependent switching process (than that provided by Equation 3.4 which calculates the efficiency in terms of input power in the core) can be obtained by calculating the amount of the optical power actually located in the graphene. Since the transmission of the grating at the pump wavelength $\left(\lambda_{4}\right)$ is about $10 \%$, then $90 \%$ of the input light is initially redirected into a cladding mode. Following this, for the $\mathrm{S}$ mode the fraction of the mode power in the graphene layer is $\approx 7 \times 10^{-5}$ (from Fig. 3.5). Therefore, at the maximum pump power used $(1334 \mathrm{~mW})$ there is $\approx 80 \mu \mathrm{W}$ in the graphene cross-section around the fiber, i.e. an intensity of $29 \mathrm{~kW} / \mathrm{cm}^{2}$. The bistable explanation is also supported by the absence of modulation for pumping on the short wavelength side: in this case the feedback is negative (even less power is coupled to the cladding as the resonance shifts to longer wavelengths due to heating): similar effects have been predicted and observed for Kerr-type nonlinearities in gratings and distributed feedback structures at much higher pump powers (but also much faster modulation speeds) [40-42].

Finally, the absence of modulation for P-polarized light is clear (Fig. 3.6(c)) and surprising in view of the results obtained in broadband steady state pumping. The most likely explanation is that in the case of $\mathrm{P}$-polarized light, with the fraction of cladding mode power being about half of that for S-polarized light, even at the maximum power level we could generate the absorbed power (and heating rate) fall below the threshold needed for self-starting oscillations, i.e. about $1000 \mathrm{~mW}$, below which even S-polarized light does not induce oscillations. This difference between S- and P-induced effects is confirmed by the difference in the imaginary part of their effectiv index (Fig. 3.10 of the Supporting information) and by similar observations that the light-matter interaction efficiency in 2D 
materials is stronger for HE or TE modes (light polarized in the plane of the 2D material) $[43-45]$.

\subsection{Conclusion}

An all single-mode fiber configuration for photo-thermal based bistability and self-pulsing has been investigated by using a multi-layered graphene coated TFBG and single watt level CW laser pumping. Self-starting pulsing at a rate of up to $269 \mathrm{~Hz}$, pulse width of $1.4 \mathrm{~ms}$, and modulation amplitudes larger than $50 \%$ has been observed. The pulse repetition rate was further observed to increase with pump power at a rate of $0.7 \mathrm{~Hz} / \mathrm{mW}$ with no evidence of saturation over the range of pump power available to us $(0-1.4 \mathrm{~W})$. Calculations of cladding mode overlap with the graphene indicate that the power density there is less than $80 \mu \mathrm{W}$, corresponding to an intensity of $29 \mathrm{~kW} / \mathrm{cm}^{2}$. The bistability arises from the nonlinear transmission of the system as a function of wavelength associated with positive feedback of the transmitted power on the wavelength of the high finesse spectral resonances via the rapid heating of the graphene layer. This phenomenon is akin to a saturable absorption of the core-guided light, but without direct contact between coreguided light and the absorbing material located on the cladding surface, and with relatively very little power actually being absorbed by the graphene. This demonstrated configuration can potentially be extended to any other system where the power coupled to the cladding of a TFBG would cause a change in the effective index of the cladding mode resonances, including coating materials with large nonlinear optical coefficients to change refractive indices through the much faster Kerr effect or other nonlinear effects such as two-photon absorption, self-focusing, self-phase modulation, saturable absorption [46-47]. 


\subsection{References}

1. A. A. Balandin, "Thermal properties of graphene and nanostructured carbon materials," Nat. Mater. 10, 569-581 (2011).

2. D. L. Nika, E. P. Pokatilov, A. S. Askerov, and A. A. Balandin, "Phonon thermal conduction in graphene: role of Umklapp and edge roughness scattering," Phys. Rev. B. 79, 155413 (2009).

3. A. A. Balandin, S. Ghosh, W. Z. Bao, I. Calizo, D. Teweldebrhan, F. Miao, and C. N. Lau, "Superior thermal conductivity of single-layer graphene," Nano Lett. 8(3), 902907 (2008).

4. R. Prasher, "Graphene spreads the heat," Science 328(5975), 185-186 (2010).

5. D. Schall, M. Mohsin, A. A. Sagade, M. Otto, B. Chmielak, S. Suckow, A. L. Giesecke, D. Neumaier, and H. Kurz, "Infrared transparent graphene heater for silicon photonic integrated circuits," Opt. Express 24(8), 7871-7878 (2016).

6. C. Horvath, D. Bachman, R. Indoe, and V. Van, "Photothermal nonlinearity and optical bistability in a graphene-silicon waveguide resonator," Opt. Lett. 38(23), 5036-5039 (2013).

7. X. Gan, C. Zhao, Y. D. Wang, D. Mao, L. Fang, L. Han, and J. J. Zhao, "Grapheneassisted all-fiber phase shifter and switching," Optica. 2(5), 468-471 (2015).

8. R. Chu, C. Guan, Y. Bo, J. Liu, J. Shi, J. Yang, P. Ye, P. Li, J. Yang, and L. Yuan, "Graphene decorated twin-core fiber Michelson interferometer for all-optical phase shifter and switch," Opt. Lett. 45(1), 177-180 (2020). 
9. Y. Gao, W. Zhou, X. K. Sun, H. K. Tsang, and C. Shu, "Cavity-enhanced thermo-optic bistability and hysteresis in a graphene-on-Si3N4 ring resonator," Opt. Lett. 42(10), 1950-1953 (2017).

10. X. Gan, Y. Wang, F. Zhao, C. Zhao, B. Jiang, L. Fang, D. Li, H. Wu, Z. Ren, and J. Zhao, "Graphene-controlled fiber Bragg grating and enabled optical bistability," Opt. Lett. 41(3), 603-606 (2016).

11. Z. Sun, A. Martine, and F. Wang, "Optical modulators with 2D layered materials," Nat. Photonics 10, 227-238 (2016).

12. A. Martinez, and Z. Sun, "Nanotube and graphene saturable absorbers for fiber lasers," Nat. Photonics 7, 842-845 (2013).

13. D. D. Li, J. W. Zhu, M. Jiang, D. Li, H. Wu, J. Han, Z. P. Sun, Z. Y. Ren, "Activepassive Q-switching fiber laser based on graphene microfiber," Appl. Phys. B 125, 203 (2019).

14. J. Albert, L. Y. Shao, and C. Caucheteur, "Tilted Fiber Bragg Grating Sensors," Laser Photonics Rev. 7(1), 83-108 (2013).

15. Y. Shevchenko, T. J. Francis, D. A. D. Blair, R. Walsh, M. C. DeRosa, and J. Albert, "In situ biosensing with a surface plasmon resonance fiber grating aptasensor," Anal. Chem. 83, 7027-7034 (2011).

16. T. Guo, F. Liu, X. Liang, X. H. Qiu, Y. Y. Huang, C. Xie, P. Xu, W. Mao, B. O. Guan, and J. Albert, "Highly sensitive detection of urinary protein variations using tilted fiber grating sensors with plasmonic nanocoating," Biosens. Bioelectron. 78, 221-228 (2016).

17. Y. Yuan, T. Guo, X. H. Qiu, J. H. Tang, Y. Y. Huang, L. Zhuang, S. G. Zhou, Z. H. Li, B. O. Guan, X. M. Zhang, and J. Albert, "Electrochemical surface plasmon resonance 
fiber-optic sensor: in situ detection of electroactive biofilms," Anal. Chem. 88, 76097616 (2016).

18. Z. C. Zhang, T. Guo, X. J. Zhang, J. Xu, W. P. Xie, M. Nie, Q. Wu, B. O. Guan, and J. Albert, "Plasmonic fiber-optic vector magnetometer," Appl. Phys. Lett. 108, 101105 (2016).

19. C. Caucheteur, T. Guo, F. Liu, B. O. Guan, and J. Albert, "Ultrasensitive plasmonic sensing in air using optical fibre spectral combs," Nat. Commun. 7, 13371 (2016).

20. X. J. Zhang, S. S. Cai, F. Liu, H. Chen, P. G. Yan, Y. Yuan, T. Guo, and J. Albert, "In situ determination of the complex permittivity of ultrathin $\mathrm{H}_{2}$-infused palladium coatings for plasmonic fiber optic sensors in the near infrared," J. Mater. Chem. C 6, $5161-5170$ (2018).

21. J. J. Lao, P. Sun, F. Liu, X. J. Zhang, C. X. Zhao, W. J. Mai, T. Guo, G. Z. Xiao, and J. Albert, "In situ plasmonic optical fiber detection of the state of charge of supercapacitors for renewable energy storage," Light Sci. Appl. 7, 34 (2018).

22. G. E. Villanueva, M. B. Jakubinek, B. Simard, C. J. Oton, J. Matres, L. Y. Shao, P. P. Millan, and J. Albert, "Linear and nonlinear optical properties of carbon nanotubecoated single-mode optical fiber gratings," Opt. Lett. 36, 2104-2016 (2011).

23. L. Y. Shao, M. B. Jakubinek, T. Sun, B. Simard, and J. Albert, "Four-wave mixing in carbon nanotube-coated optical fiber gratins," Appl. Phys. Lett. 100, 071108 (2012).

24. F. Liu, and J. Albert, "40 GHz-rate all-optical cross-modulation of core-guided near infrared light in single mode fiber by surface plasmons on gold-coated tilted fiber Bragg gratings," APL Photon. 4, 126104 (2019). 
25. J. Albert, D. Vincent, and R. Tremblay, "Hybrid bistable optical device using an acoustooptic waveguide modulator," Can. J. Phys. 59, 1251-1253 (1981).

26. H. M. Gibbs, Optical Bistability: Controlling light by light (Academic, 1985).

27. C. M. D. Sterke, J. E. Sipe, "Switching dynamics of finite periodic nonlinear media: a numerical study," Phys. Rev. A 42(5), 2858-2869 (1990).

28. C. M. D. Sterke, "Theory of modulational instability in fiber Bragg gratings," J. Opt. Soc. Am. B 15(11), 2660-2667 (1998).

29. I. V. Kabakova, D. Grohnic, S. Mihailov, E. C. Magi, C. M. D. Sterke, B. J. Eggleton, "Bragg grating-based optical switching in a bismuth-oxide fiber with strong $\chi^{(3)}$ nonlinearity," Opt. Express 19(7), 5869-5873 (2011).

30. M. Z. Alam and J. Albert, "Selective excitation of radially and azimuthally polarized optical fiber cladding modes," J. Lightw. Technol. 31(19), 3167-3175 (2013).

31. M. Qi, Y. Zhou, F. Hu, X. Xu, W. Li, A. Li, J. Bai, and Z. Ren, "Improving terahertz sheet conductivity of graphene films synthesized by atmospheric pressure chemical vapour deposition with acetylene," J. Phys. Chem. C 118(27), 15054-15060 (2014).

32. M. Qi, Z. Ren, Y. Jiao, Y. Zhou, X. Xu, W. Li, J. Li, X. Zheng, and J. Bai, "Hydrogen kinetics on scalable graphene growth by atmospheric pressure chemical vapor deposition with acetylene," J. Phys. Chem. C 117(27), 14348-14353 (2013).

33. S. Radic, N. George, and G. P. Agrawal, "Optical switching in $\lambda / 4$-shift nonlinear periodic structures," Opt. Lett. 19, 1789-1791 (1994).

34. S. Radic, N. George, and G. P. Agrawal, "Theory of low-threshold optical switching in nonlinear phase-shift periodic structures," J. Opt. Soc. Am. B 12(4), 671-680 (1995). 
35. N. Mattiucci, M. J. Bloemer, and G. D. Aguanno, "All-optical bistability and switching near the Dirac point of a 2-D photonic crystal," Opt. Express 21(10), 11862-11868 (2013).

36. T. Gu, N. Petrone, J. F. McMillan, A. V. D. Zande, M. Yu, G. Q. Lo, D. L. Kwong, J. Hone, and C. W. Wong, "Regenerative oscillation and four-wave mixing in graphene optoelectronics," Nature Photon. 6, 554-559 (2012).

37. T. Christensen, W. Yan, A. P. Jauho, M. Wubs, and N. A. Mortensen, "Kerr nonlinearity and plasmonic bistability in graphene nanoribbons," Phys. Rev. B 92 , $121407(2015)$.

38. R. Li, H. Wang, B. Zheng, S. Dehdashti, E. Li, and H. Chen, "Bistable scattering in graphene-coated dielectric nanowires," Nanoscale 9, 8449-8457 (2017).

39. A. Yariv, Quantum Electronics (2nd ed.) (Wiley: New York, 1975).

40. H. G. Winful, J. H. Marburger, E. Garmire, "Theory of bistability in nonlinear distributed feedback structures," Appl. Phys. Lett. 35(5), 379-381 (1979).

41. H. G. Winful, G. D. Cooperman, "Self-pulsing and chaos in distributed feedback bistable optical devices," Appl. Phys. Lett. 40(4), 298-300 (1982).

42. Q. L. Bao, H. Zhang, B. Wang, Z. H. Ni, C. H. Y. X. Lim, Y. Wang, D. Y. Tang, and K. P. Loh, "Broadband graphene polarizer," Nature Photon. 5, 411-415 (2011).

43. J. T. Kim, and C. G. Choi, "Graphene-based polymer waveguide polarizer," Opt. Express 20(4), 3556-3558 (2012).

44. X. Yin, T. Zhang, L. Chen, and X. Li, "Ultra-compact TE-pass polarizer with graphene multilayer embedded in a silicon slot waveguide," Opt. Lett. 40(8), 1733-1735 (2015). 
45. F. Bonaccorso, Z. Sun, T. Hasan, and A. C. Ferrari, "Graphene photonics and optoelectronics," Nature Photon. 4, 611-622 (2010).

46. A. Parini, G. Bellanca, S. Trillo, M. Conforti, A. Locatelli, C. D. Angelis, "Self-pulsing and bistability in nonlinear Bragg gratings," J. Opt. Soc. Am. B 24(9), 2229-2237 (2007).

47. Q. L. Bao, and K. P. Loh, "Graphene photonics, plasmonics, and broadband optoelectronic devices," ACS Nano. 6(5), 3677-3694 (2012). 


\subsection{Supplementary information}

\subsubsection{Supplementary note 1: Experiment configuration}
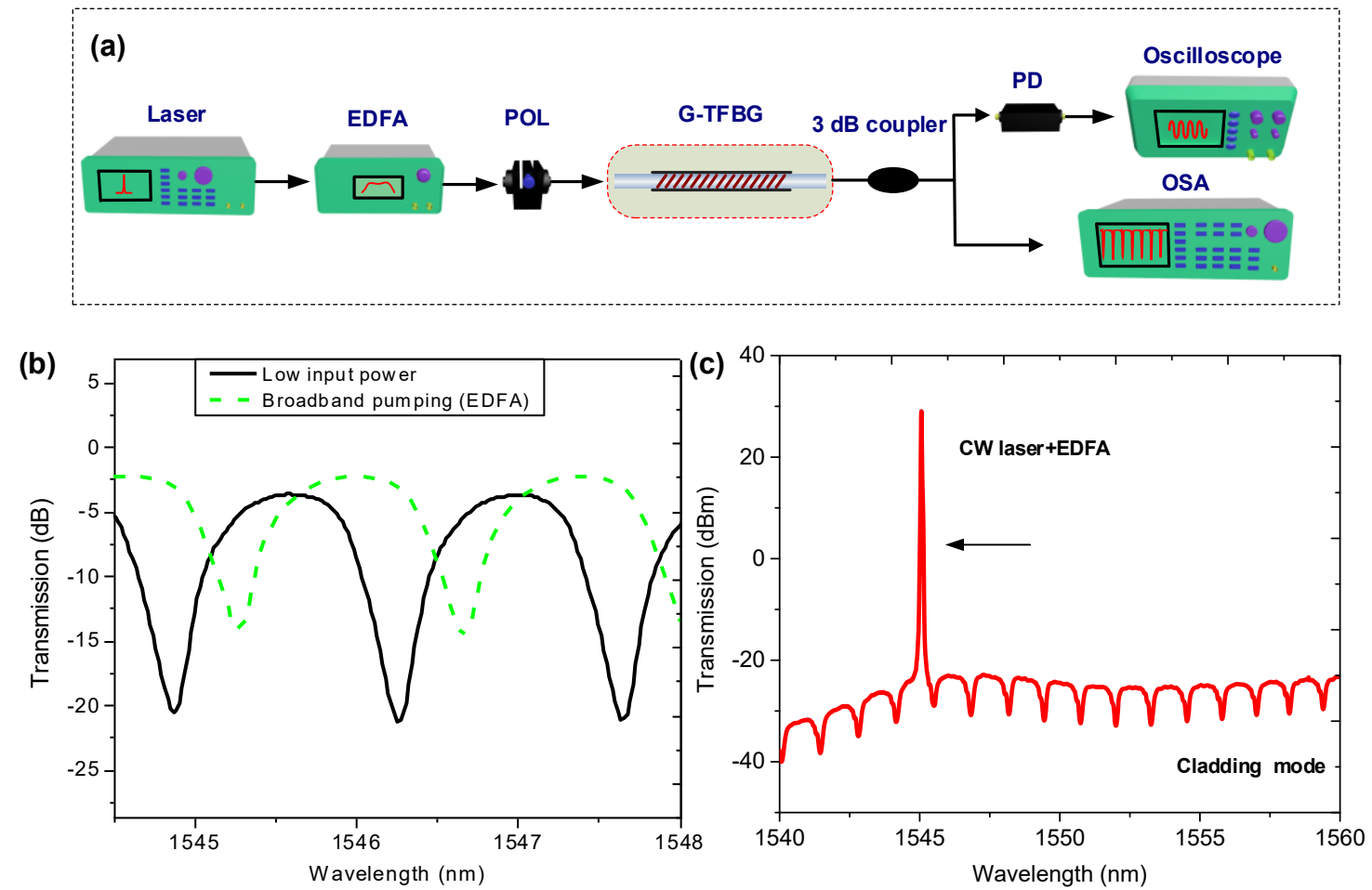

Fig. 3.9. Schematic diagram of the switching system; (b) G-TFBG spectrum changes during nonresonant pumping. The dashed green spectrum represents both the stable output power resulting from non-resonant pumping, and the instantaneous one produced by resonant pumping; (c) resonant pumping spectrum.

\subsubsection{Supplementary note 2: dynamic of the self-starting modulated switching}

The model for self-pulsing is based on a simplified one-dimensional finite-difference simulation of the temperature on a $125 \mu \mathrm{m}$ long spatial grid corresponding to a diameter of the coated fiber using the thermal diffusion equation and a time-dependent heat source at the two ends of the simulation domain, which correspond to the fiber surface [S1]: 


$$
\frac{\partial^{2} \Delta T}{\partial^{2} r}=\frac{\partial \Delta T}{\alpha \cdot \partial t}
$$

Where $\Delta T$ is the temperature rise due to graphene heating, $r$ is the radial coordinate, and $\alpha$ is an equivalent diffusivity parameter for the system. The system is solved in Matlab by using the finite differential form of Equation 3.5 (on a spatial grid of $r$ values separated by $\Delta \mathrm{r})$ :

$$
\frac{\mathrm{T}^{\mathrm{t}+1}-\mathrm{T}^{\mathrm{t}}}{\Delta \mathrm{t}}=\alpha \frac{\left(\mathrm{T}^{\mathrm{x}+1, \mathrm{t}}+\mathrm{T}^{\mathrm{x}-1, \mathrm{t}}-2 \mathrm{~T}^{\mathrm{x}, \mathrm{t}}\right)}{(\Delta \mathrm{r})^{2}}
$$

Where the equivalent diffusivity parameter $\alpha$ has been adjusted to fit the experimental results as:

$$
\alpha=5.05 \times 10^{-8}+1.35 \times 10^{-6} /\left(1+10^{5.353\left(1.135-\mathrm{P}_{\text {in }}\right)}\right) \mathrm{m}^{2} / \mathrm{s}
$$

In Equation 3.5 the temperature at the surface is increased at each time step by an amount derived from Equation 3.1 and 3.2 where $F_{\text {clad }}$ is determined from standard coupled-mode theory as [S2]:

$$
\mathrm{F}_{\text {clad }}=\left|\frac{\kappa \cdot \sinh (\mathrm{SL})}{-\Delta \beta \cdot \sinh (\mathrm{SL})+\mathrm{iS} \cdot \cosh (\mathrm{SL})}\right|^{2} \text { with } \mathrm{S}=\sqrt{\kappa^{2}-(\Delta \beta+\delta(\Delta \beta))^{2}}
$$

and where

$$
\Delta \beta=(2 \pi / \lambda)\left(\mathrm{N}_{\text {eff }}(\text { core })+\mathrm{N}_{\text {eff }}(\text { clad })\right)-2 \pi / \Lambda
$$

is the detuning of the input light from the resonance before heating comes into play. Furthermore,

$$
\kappa=\tanh ^{-1} \sqrt{1-\mathrm{T}_{\min }} / \mathrm{Lg}
$$


is the coupling constant of the grating derived from the maximum amplitude of the core mode loss at resonance $\left(\mathrm{T}_{\min }\right)$ while the change in detuning of the resonance by the temperature change in the glass is derived from Equation 3.3 as:

$$
\delta(\Delta \beta)=\frac{4 \pi}{\lambda} \frac{\mathrm{dn}}{\mathrm{dT}} \Delta \mathrm{T}(\text { fiber })
$$

\subsubsection{Supplementary note 3: Imaginary part of graphene coating-guided cladding} mode

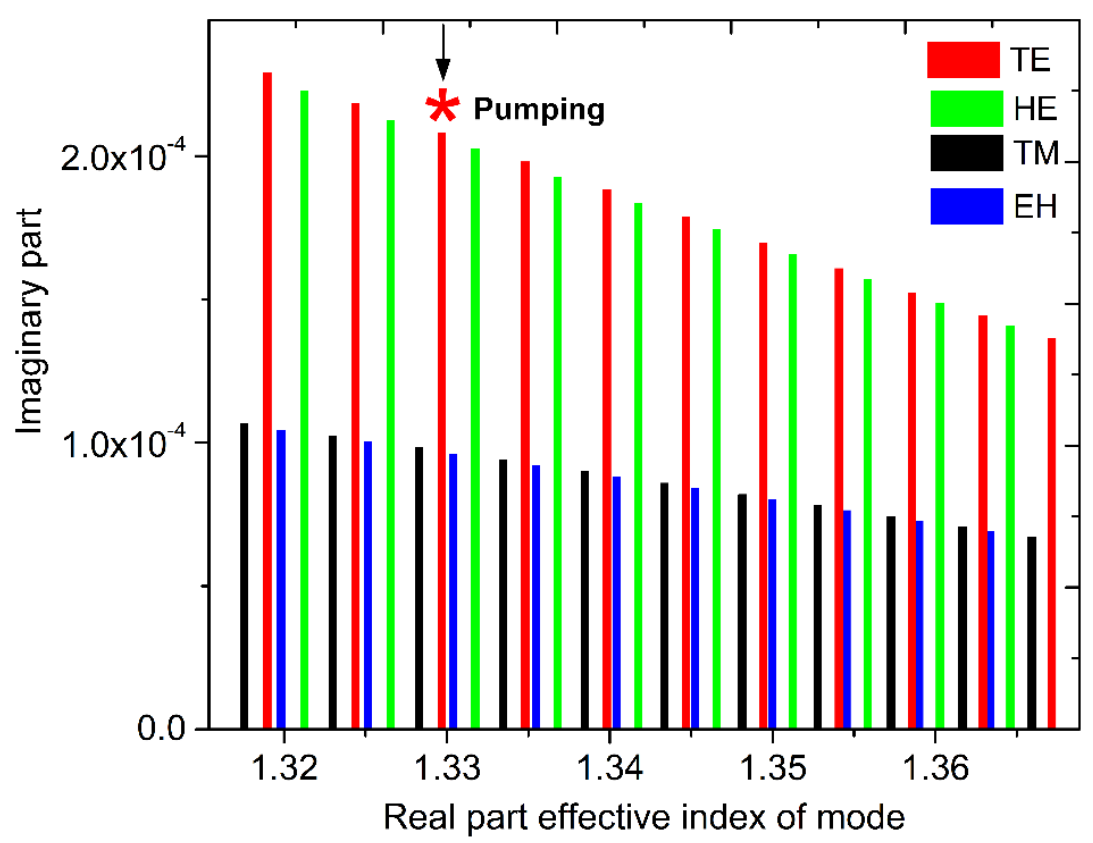

Fig. 3.10. Simulation of the attenuation of graphene coating-guided cladding modes with effective indices near 1.33 corresponding to $\mathrm{CW}$ pumping position. Each vertical bar corresponds to a particular cladding mode and is color-coded by its polarization state (Red and green for TE and HE modes, which are S-polarized at the cladding surface; Black and blue for TM and EH mode, which are P-polarized).

Fig. 3.10 shows the effective refractive index of graphene coating-guided cladding mode by FIMMWAVE mode solver by setting: $8.2 \mu \mathrm{m}$ core diameter with refractive index 
1.449311, $125 \mathrm{~mm}$ cladding diameter with refractive index $1.444078,6 \mathrm{~nm}$ thick graphene thickness with complex refractive index 2.52-2.24i [S3], and surrounding medium of air (refractive index $=1.00027$ ). The imaginary part of guided mode (which determines the absorption of cladding guided mode power by the graphene overlayer and induces the thermal nonlinearity) rises gradually towards smaller mode effective indices (i.e. higher order modes and corresponding lower wavelength resonances in the TFBG spectrum) [S4S6]. Because of the 2D nature and thinness of the graphene coating, the absorption efficiency for S polarized input is more than two times larger than that of $\mathrm{P}$ polarized at the mode where the effective index is around 1.33 , corresponding to resonances where $\mathrm{CW}$ pumping is performed, and the mode fill factor is $7.4 \times 10^{-5}$ in the graphene layer (HE mode).

\subsection{Supplementary References}

S1. J. P. Holman, Heat Transfer (10nd ed.), McGraw-Hill, New York 2010.

S2. A. Yariv, Quantum Electronics (2nd ed.), Wiley, New York 1975.

S3. X. Gan, C. Zhao, Y. D. Wang, D. Mao, L. Fang, L. Han, and J. J. Zhao, "Grapheneassisted all-fiber phase shifter and switching," Optica. 2(5), 468-471 (2015).

S4. C. Caucheteur, T. Guo, F. Liu, B. O. Guan, and J. Albert, "Ultrasensitive plasmonic sensing in air using optical fibre spectral combs," Nat. Commun. 7, 13371 (2016).

S5. W. J. Zhou, Y. Zhou, and J. Albert, "A true fiber optic refractometer," Laser Photonics Rev 11(1), 1600157 (2017).

S6. X. Chen, J. Xu, X. Zhang, T. Guo, and B. O. Guan, "Wide range refractive index measurement using a multi-angle tilted fiber Bragg grating," IEEE Photon. Technol. Lett. 29(9), 719-722 (2017). 


\title{
Chapter 4: $40 \mathrm{GHz}-$ rate all-optical cross-modulation of core-guided near infrared light in single mode fiber by surface plasmons on gold-coated tilted fiber Bragg gratings
}

\begin{abstract}
All-optical control of Surface Plasmon Polaritons (SPPs) can switch light with high speed and large signal to noise ratio. We demonstrate 25 ps-timescale switching of continuous wave light by pump pulses co-propagating in the same single mode fiber at different wavelengths near $1550 \mathrm{~nm}$. The switching is due to hot carriers from the SPPs in a $45 \mathrm{~nm}$-thin gold coating on the fiber cladding. The SPPs are generated by pump pulses coupled from the core to cladding modes by a tilted fiber Bragg grating (TFBG). Hot carriers modify the complex permittivity of the metal coating on a time scale of the order of picoseconds and hence the cladding mode resonance wavelengths of the TFBG. A probe light signal co-propagating in the same fiber can therefore be modulated by the transmission resonance shifts. With 25 ps pulses at $1540.4 \mathrm{~nm}, 1 \mathrm{MHz}$ and $50 \mathrm{~mW}$ average power, the modulation depth of a $\mathrm{CW}$ probe at $1543.4 \mathrm{~nm}$ co-propagating in the core reached $4.5 \pm 1 \%$ with a pulse width broadened to $56 \mathrm{ps}$. Under these conditions, the pump power density was $0.147 \mathrm{GW} / \mathrm{cm}^{2}$ in the metal layer, for a conversion efficiency as high as $30 \pm 7 \%$ per $\mathrm{GW} / \mathrm{cm}^{2}$. Since many other plasmonic and nonlinear active materials can be deposited on fiber claddings, we believe that this very simple all-fiber configuration to perform all-optical switching of core-guided light in single mode fibers by plasmonmodulated resonances has strong potential applications in studies of light-matter interactions over fast and ultrafast time scales.
\end{abstract}




\subsection{Introduction}

Surface Plasmons Polaritons (SPP) provide an effective way to confine electro-magnetic fields to sub-micrometer thick layers near the surface of metals and excitation of SPP at femtosecond time scales can initiate a cascade of linear and nonlinear processes with multiple outcomes such as non-radiative decay to carriers and phonons, radiative decay via emission of photons [1-4]. Such processes have opened new avenues toward the realization of ultrafast nanophotonic devices for all-optical signal processing in high speed telecommunication networks but one of the major challenges is how to achieve fast and efficient switching or modulation of light by SPPs without excessive insertion losses in fiber or optical waveguide systems. In earlier work, plasmonic devices combining phase control in external materials like semiconductor [5,6] or organic films [7] achieved light switching or modulation but on relatively long timescales of microseconds because of dominant thermal processes. When generating SPPs by interband transition pulsed pumping on the other hand, hot carriers are generated on femtosecond time scales in the metal and they strongly modify the complex permittivity on the same time scale [8-12]. Such changes can then be used to build modulators and switches from nonlinear effects in guided wave interferometers and resonators operating at sub-picosecond time scales [1315]. The issue with interband pumping is that it necessitates pump photons at wavelengths much shorter than the telecommunication bands in the near infrared. To avoid this problem, intraband (using longer wavelength photons) transitions in so called "epsilon near zero" material have been identified as extraordinary new building blocks for high performance optical devices with extremely high nonlinearity and ultrafast switching speed covering the near- and mid-infrared wavelength ranges [16-19]. Again, integration of epsilon near zero 
materials with optical fibers remains challenging for now and it is desirable to find devices and configurations where hot carriers from intraband pumping could be used. It has been shown that hot carriers generated by SPPs at near-infrared wavelengths in "conventional" metals can be used for switching but with modulation efficiencies is much lower than those achieved by interband transitions [20-22]. Therefore, designing practical switches with GHz-level bandwidths and high modulation efficiencies at telecommunication wavelengths remains an important goal for photonic and materials research.

One of the ways in which light guided in the core of standard single mode fibers can be made to interact with materials deposited on the surface of the cladding is through TFBG written in the fiber core by photosensitive processes [23]. The transmission spectrum of TFBGs contains hundreds of "comb"-like discrete resonances corresponding to coupling from a forward propagating core mode light to backward propagating cladding modes. Under certain conditions, when the cladding is covered by a thin metal coating, some of the cladding modes have effective indices that are closely matched to the effective index of an SPP mode propagating at the outer surface of the metal coating. When this occurs, and when the metal layer is thin enough for the evanescent field of the cladding modes to tunnel through the metal layer, some of the cladding mode power is coupled to the SPP, resulting in increased attenuation of the cladding mode and some broadening of the corresponding resonances [23-25]. It is important to note that the TFBG resonances have a Q-factor greater than $10^{4}$ and their signal to noise ratio can be greater than $40 \mathrm{~dB}$, with near zero insertion loss out of the resonances. Because of the large spectral slopes of the cladding mode resonances, small perturbations of the cladding mode properties (such as those due to permittivity changes occurring near the outer metal surface) can lead to large 
changes in transmission at certain wavelengths, by $10 \mathrm{~s}$ of $\mathrm{dB}$ in certain cases thus allowing the development of high-performance sensors in multiple areas such as biomedicine [2628], electrochemical and magnetic detection [29,30], gas monitoring [31,32], and renewable energy [33]. Furthermore, these features are obtained with very simple device fabrication method and trivial, very low loss input/output coupling. Therefore, such devices provide an ideal tool to study light-matter interaction processes and to develop optical switching systems.

In this chapter, an all-optical 25 ps-scale switching configuration based on TFBGassisted plasmonics in a gold coated standard single mode optical fiber is proposed and demonstrated. Pumping one of the SPP-active cladding modes with intense laser pulses launched in the fiber core leads to an increase in the damping term in the dielectric function of the gold and consequently to a synchronous (due to the femtosecond response time for the generation of hot carriers) change in the SPP effective index. This in turn shifts the SPP spectrum as well as the underlying cladding mode resonances. When a second (continuous wave $(\mathrm{CW})$ ) signal is injected in the core of the same fiber (with a wavelength-multiplexing fiber coupler) at a wavelength associated with another cladding mode resonance (but still within the spectral bandwidth of the SPP), the latter signal is modulated by the pumpinduced optical property changes in the metal coating, on the same time scale as that of the pump. We achieved the modulation of a $\mathrm{CW}$ probe signal with $\sim 56 \mathrm{ps}$ response time and switching efficiency as high as $30 \pm 7 \%$ per $\mathrm{GW} / \mathrm{cm}^{2}$. 


\subsection{Method}

\subsubsection{Fabrication of the TFBG}

The TFBGs (20 mm in length) were inscribed in hydrogen-loaded CORNING SMF-28 fibers by using the phase-mask method [23]. The fibers were hydrogen-loaded at a pressure of 15.2 $\mathrm{MPa}$, a temperature of $20^{\circ} \mathrm{C}$, and a duration of 14 days, conditions sufficient to saturate the fiber core and increase its photosensitivity to ultraviolet light. After stripping a $5 \mathrm{~cm}$ section of fiber jacket, gratings were inscribed in the stripped section with a pulsed high-energy excimer laser (model PM-848 from Light Machinery Inc.) and the phase mask technique. The laser was operating at $248 \mathrm{~nm}$ with a fluence per pulse at the fiber of $\sim 40$ $\mathrm{mJ} / \mathrm{cm}^{2}$ over an area of $2 \mathrm{~cm} \times 50 \mu \mathrm{m}$ determined by selecting a portion of the laser beam and focusing it along the fiber. The tilt of the grating fringes was obtained by rotating the fiber/phase mask assembly (as well as the cylindrical focusing lens) around an axis perpendicular to the fiber axis and the plane of incidence.

\subsubsection{Fabrication of the gold coating}

A $45 \mathrm{~nm}$ thick uniform gold film (supplementary note 1) was deposited on the TFBG by thermal evaporation. To achieve a high-quality coating, the stripped fiber section is cleaned by immersing it in a piranha solution (an 8:1:1 mixture of deionized water, Ammonium Hydroxide, and Hydrogen Peroxide) for 30 minutes. Then the TFBG was fixed on a movable holder in a thermal-evaporation physical vapor deposition system (Balzers BA $510)$ at room temperature and under vacuum $\left(10^{-7}\right.$ Torr $)$. Two deposition steps were carried out, with a rotation of the fiber holder by $180^{\circ}$ between the two runs. The depositions were carried out at a relatively rapid deposition rate of $6 \mathrm{~nm} / \mathrm{min}$ for 7.5 minutes per side, 
resulting in a mass-equivalent thickness of $45 \mathrm{~nm}$ around the full circumference of the fiber.

\subsubsection{Dynamic TFBG-SPR simulations}

In the simulations of TFBG-SPR spectra modulated by hot carriers decay, three steps are considered for the ultrafast switching process. Firstly, the temporal evolution of hot electron populations and lattice temperature due to plasmonic excitation of the gold film were simulated by using a delayed two-temperature model within the short pumping time (supplementary note 2). Secondly, the resulting change in the dielectric function (complex permittivity) of the gold layer was calculated by using a Drude model with two critical points and damping terms modulated by hot electron and phonon temperatures (supplementary note 3). Finally, the time-dependent TFBG-SPR spectra associated with the variations of the dielectric function of the gold layer were simulated by first calculating the changes in the radially polarized cladding mode families (i.e. the EH and TM mode families, which are the only ones that can hybridize with SPPs of a metal layer on the cladding) with a cylindrical finite-difference vector mode solver at each wavelength (and taking into account material dispersion), and then using complex coupled-mode theory and a Runge-Kutta algorithm to calculate the transmission at each wavelength.

\subsubsection{Ultrafast switching measurement}

As shown in Fig. 4.9 (supplementary note 4), the pump-probe interrogation setup consists of a tunable picosecond laser (Genia Photonics Programmable Picosecond Laser, pulse width: $25 \mathrm{ps}$; repetition rate $1 \mathrm{MHz}$; adjustable average power: 0-100 $\mathrm{mW}$ ) and a CW tunable laser (HP 81640A tunable laser, power: $2 \mathrm{~mW}$ ) coupled into a common fiber with a $3 \mathrm{~dB}$ coupler. A manually adjustable bandpass filter (Yenista XTM-50) was used 
downstream of the TFBG to prevent the pumping pulse from reaching the detector. The pump modulated probe light was detected by a $40 \mathrm{GHz}$ photodetector (New Focus, Inc) and measured with a digital oscilloscope (86100D Infiniium DCA-X Wide-Bandwidth Oscilloscope). Pump and probe wavelengths were adjusted precisely relative to the TFBG resonances by directing the output of the TFBG to an Optical Spectrum Analyzer (ANDO AQ 6317B) instead of the photodiode, in between switching experiments.

\subsection{Results}

\subsubsection{The hybrid SPP-cladding modes of a TFBG}

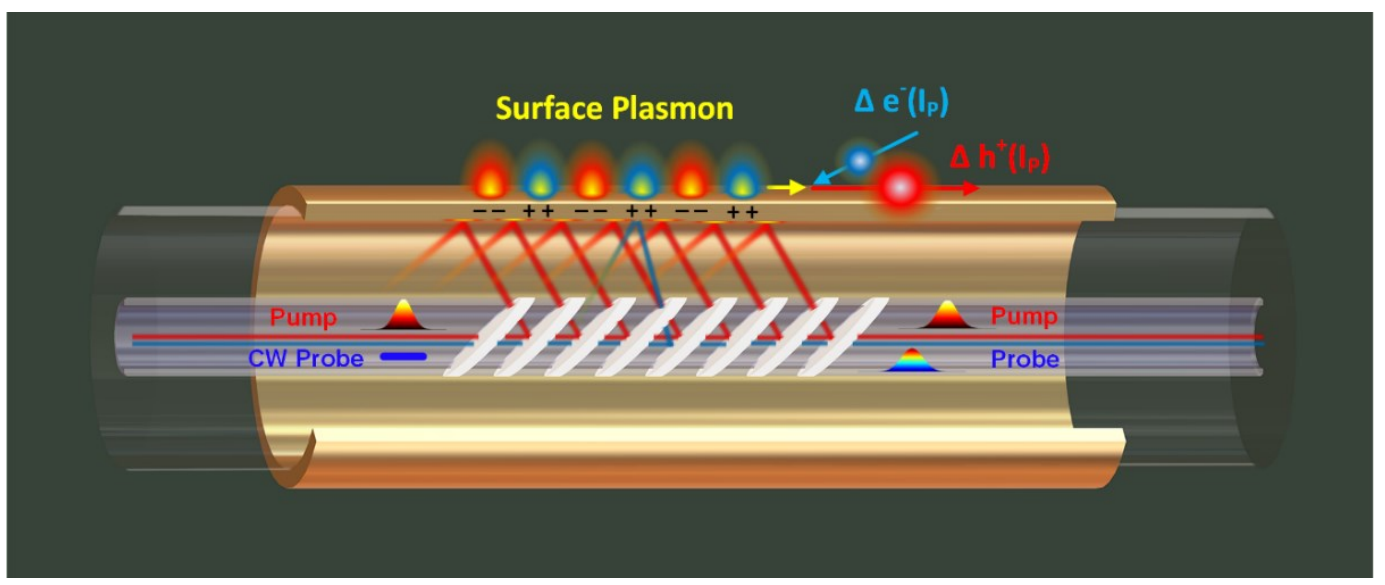

Fig. 4.1. Schematic diagram of the plasmonic gold-coated TFBG. The pulsed pump $\left(I_{P}\right)$ coupled out of the core by the tilted grating excite SPP on the gold surface and hot carriers $\left(\mathrm{e}^{-}\right.$and $\left.\mathrm{h}^{+}\right)$that in turn modulate the metal permittivity. A co-propagating $\mathrm{CW}$ probe at a different wavelength gets modulated as the grating resonances shift in response to the permittivity change.

The effective index of the SPP propagating at the interface of two media with relative permittivity $\varepsilon_{1}$ and $\varepsilon_{2}$ is given by [1]:

$$
N_{e f f}^{S P P}=\sqrt{\frac{\varepsilon_{1} \varepsilon_{2}}{\varepsilon_{1}+\varepsilon_{2}}}
$$


At near infrared wavelengths in the vicinity of $1550 \mathrm{~nm}$, the relative permittivity for gold and water are -115.13-11.259i and 1.7371-0.0000986i respectively, yielding $\mathrm{N}_{\mathrm{eff}}^{\mathrm{SPP}}$ values near $1.328[34,35]$. Then, in order to excite the SPP at the surface of a gold coating deposited on the cladding of an optical fiber immersed in water and remembering that the cladding supports guided mode with effective indices between the index of silica glass (near 1.444 at these wavelengths) and that of the external medium (1.315), it is sufficient to use an in-core tilted grating to couple light from the single mode core to a cladding mode that has an effective index equal to $\mathrm{N}_{\mathrm{eff}}^{\mathrm{SPP}}$. To be precise, a $556.015 \mathrm{~nm}$ period grating with an internal tilt angle of 12 degrees can generate a large number of cladding modes with effective indices in the vicinity of the required value at wavelengths near $1550 \mathrm{~nm}$, as will be shown in exact calculations below. So even though the light wave frequency is far from the plasma frequency ( $0.8 \mathrm{eV}$ vs $2.38 \mathrm{eV}$ in the usual units for plasmonics) the real part of the permittivity of gold has a large negative value and a SPP wave can be excited efficiently by the evanescent wave of a cladding mode tunnelling across the gold layer. With such low energy photon excitation, SPP excitation relies on phonons to provide the necessary momentum conservation to generate the hot carriers. These phonon-assisted transitions are important contributors to losses arising from various damping mechanisms such as a Landau damping and electron-phonons scattering which add up to contributions from electron-electron scattering and losses arising from defects of the metal such as nanoscale roughness [20-22]. 

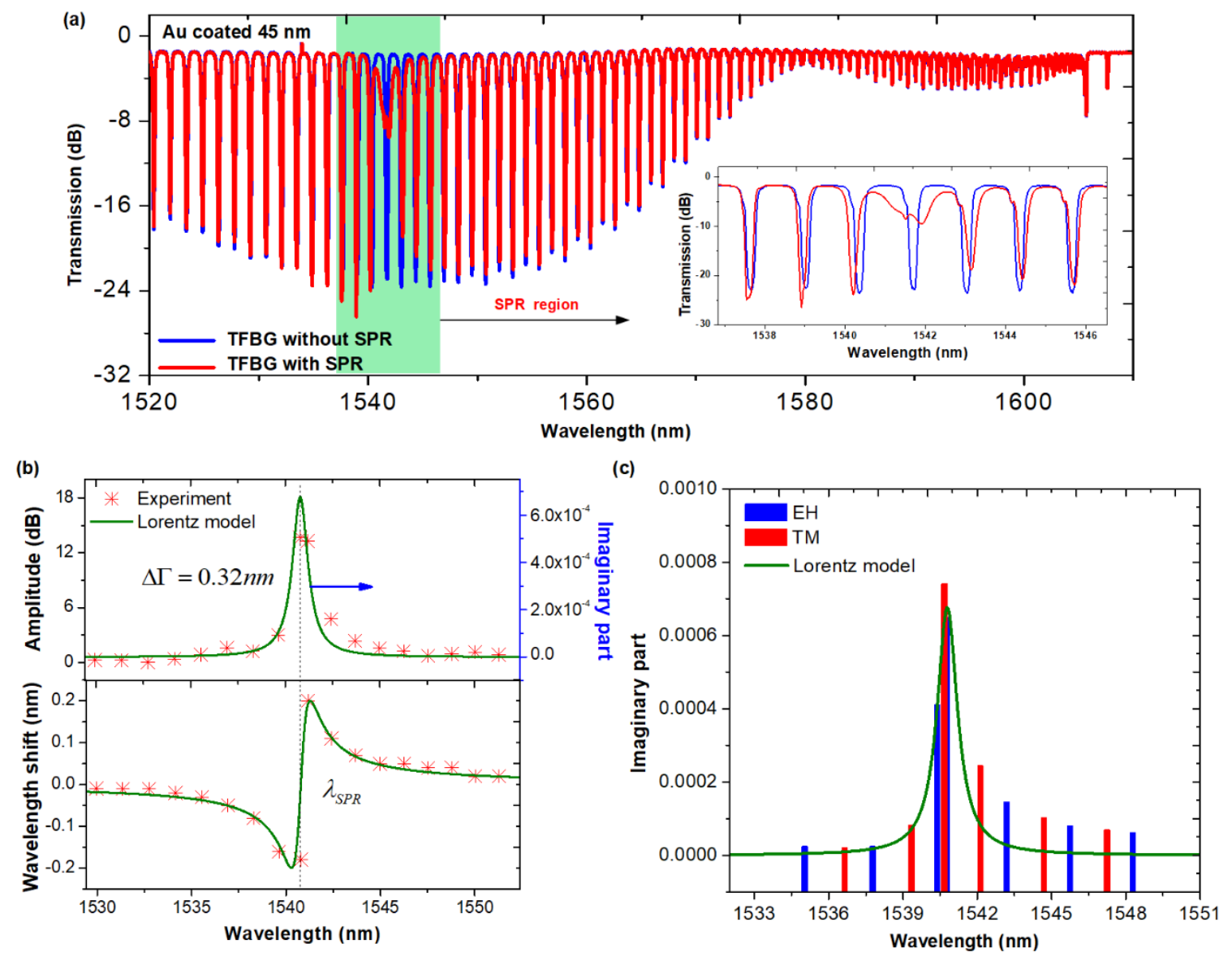

Fig. 4.2. TFBG-SPR linear properties: (a) measured spectrum in water (red, SPP phase matching near $1540 \mathrm{~nm}$ ) and air (blue, no surface plasmon at these wavelengths); (b) the Lorentz model fitting for wavelength shifts with- and without- SPR (indicative of changes in the real part of the cladding mode effective index), and of the amplitude change (due to the imaginary part of the mode effective index); (c) The relationship between the Lorentz fit of (b) and the values of the imaginary part of the effective index of guided cladding modes calculated using FIMMWAVE close to SPR (the TM/EH modes are those excited by P-polarized input light). Non SPR-active modes have imaginary parts of the order of $10^{-5}$ and less at the same wavelengths.

Fig. 4.2 shows the transmission spectra of a $12^{\circ}$ tilted Au-coated, 2-cm long TFBG measured in air and immersed in deionized water. The main feature of these spectra is the presence of a very dense comb of cladding mode resonances that are individually very 
narrow (sub-nm). In both cases, in order to fulfill the necessary condition for the excitation of SPP waves of the gold film, the input light injected in the core is polarized in the plane of the tilt (P-polarized) which results in the excitation of cladding modes that are radially polarized at the outsides surface of the fiber (i.e. TM-like) [36]. When the TFBG is in air, the index contrast is highest, the cladding modes are well confined in the fiber by the 45 $\mathrm{nm}$ thick gold coating and the spectrum is not much different from that of the same TFBG in a bare fiber. However, when the TFBG is immersed in water, the lower index contrast allows some tunneling of energy to the external surface of the gold and to the excitation of a SPP at wavelengths near $1541 \mathrm{~nm}$ where cladding modes have effective indices near that of pure SPP waves of a gold-water interface. For S-polarized input, no SPP can be excited even in water because the cladding mode polarization is then TE-like. When a SPP is excited, the mode power fraction in water increases from 0.68 to $25.7 \%$ and in the metal layer from 0.035 to $0.736 \%$ (supplementary note 5 , Fig. 4.12). This has two consequences: shifts in the resonance wavelengths and resonance amplitude decreases of those cladding modes with effective indices within the SPP spectral width (see the inset of Fig. 4.2(a)). Those cladding modes become hybridized with the SPP. The measured resonance modifications are reported in Fig. 4.2(b) and fitted by a Lorentz line shape function of the mode effective indices (supplementary note 6) where the real and imaginary parts are associated respectively with wavelength shifts (directly caused by change in the real part of the mode effective index, through the grating phase matching condition) and with transmission changes (since the increase in the imaginary part, i.e. loss, of the mode effective indices shortens the effective coupling length and hence the reflectivity of the resonance). A Lorentzian fit of the shifts gives a SPP-hybridization width of $0.32 \mathrm{~nm}$ for 
the metal coated fiber in Fig. 4.2(c). As in other surface plasmon resonance phenomena, the hybridization width is strongly dependent on the metal thickness [37] (supplementary note 7, Fig. 4.13). Here, the $44 \mathrm{~nm}$ thickness and very low surface roughness (near $1 \mathrm{~nm}$ ) result in a very narrow lossy bandwidth where collective collision damping is the dominant source of loss [38]. In these conditions, only 1 or 2 cladding mode resonances are strongly attenuated. While these particular resonances are rendered "useless" by the excessive loss and consequential weakening of the coupling, adjacent resonances retain enough coupling to the SPP while maintaining significant coupling with the core mode as well as a narrow linewidth required for efficient switching.

In order to demonstrate the validity of the Lorentz model for absorption, the complex effective indices of the hybridized cladding guided-SPP modes of the Au-coated fiber were simulated using the FIMMWAVE mode solver (from Photon Design Inc.). By use of the grating phase matching relation, the simulation results can be plotted as the mode loss (imaginary part of the effective mode index) against wavelength, as shown in Fig. 4.2(c). The imaginary part of the cladding mode effective index peaks at a value of 0.0007 close to the SPP resonance and fits the Lorentzian line-shape of the measured resonance loss increases. At the peak, the loss corresponds to an attenuation coefficient of $57 \mathrm{~cm}^{-1}$ for the cladding mode centered on the SPR wavelength, much too large for efficient coupling by the $2 \mathrm{~cm}$-long grating. Pumping on the nearest neighbor resonance however still couples energy to the SPP but with a cladding mode that has an attenuation coefficient 10 times smaller (of the order of $6 \mathrm{~cm}^{-1}$ ) which allows for strong coupling from the input core mode.

While this excitation condition is relatively narrowband its consequences following intense in-band pumping of one resonance affect all the other cladding mode resonances 
within the SPP bandwidth due to the global permittivity change induced by the hot carriers. Therefore, probe light at neighboring resonances will experience the consequences of pumping (i.e. modulation) at some wavelength separation from the pump wavelength and peak SPP coupling without the excessive damping losses experienced by in-band light.

\subsubsection{Ultrafast dynamic of surface plasmon decay}

In noble metals, it was demonstrated that internal thermalization of the electrons (i.e., establishment of an electron temperature following some excitation mechanism) takes place on the time scale of a few picoseconds thereby allowing ultrafast switching mechanisms to take place [39-42]. The change in temperature of the conduction band electrons modifies the Fermi-Dirac distribution function, which decreases the population below the Fermi level and increases it above the Fermi level. As a result, the response time and the nonlinear susceptibility of hot electrons in metals in terms of plasmonics have been widely investigated [43-45]. In particular, hot carriers generated by surface plasmon decay in thin metal films [20-22] make it possible to achieve ultrafast switching based on the modulation of high Q-factor transmission resonances in TFBGs. Fig. 4.3 shows a simulation of the effect of the dynamic hot carriers generated by picosecond pulsed excitation of SPP on the transmission spectrum of a TFBG. 

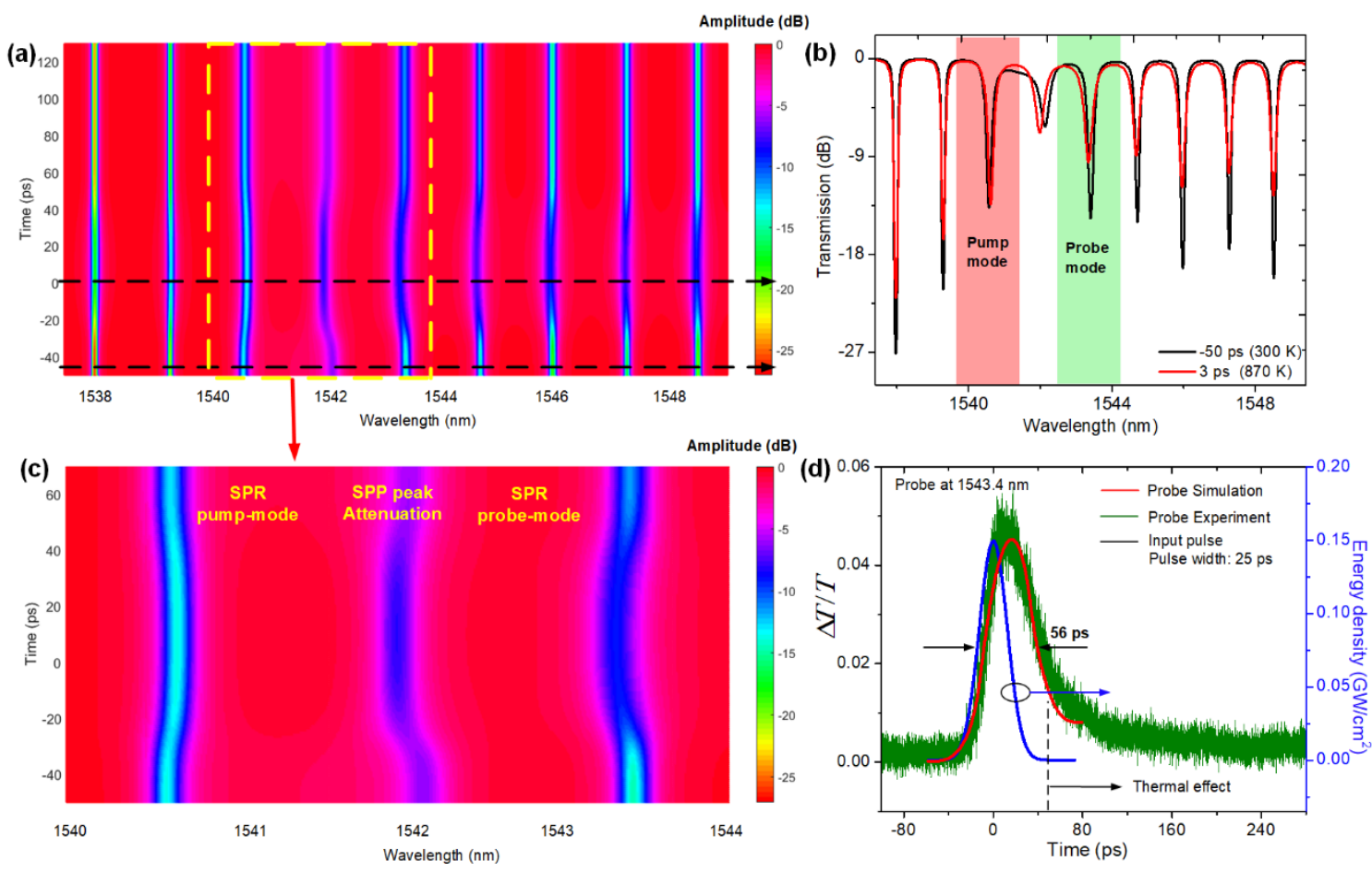

Fig. 4.3. Plasmonic ultrafast switching based on TFBG spectral combs: (a) simulated spectrum evolution during the hot carrier dynamic process (Supplementary Video 1); (b) simulated spectra for electrons at room temperature (black line) and hot electron temperature (870 K, red line); (c) dynamics of cladding modes close to SPR; (d) experimental ultrafast switching of the probe light (Supplementary Video 2).

A delayed two-temperature model for the hot electrons (supplementary note 2, Fig. 4.7) is used to calculate the non-thermal energy dissipation by electron-electron scattering and by reaching thermal equilibrium via electron-phonon scattering. This results in a timedependent change in the damping term of the imaginary part of the complex permittivity of the Au film (supplementary note 3, Fig. 4.8). The calculated permittivity as a function of time is then input into a cylindrical finite-difference complex vector mode solver to calculate perturbed mode fields and complex effective indices which are then used to calculate transmission spectra by coupled mode theory. The end result is a sequence of 
simulated transmission spectra during and after the arrival of a 25 ps duration pump pulse from time $\mathrm{t}=-50$ to $+125 \mathrm{ps}$ (Fig. 4.3(a)) and more closely during the pulse ( $\mathrm{t}=3 \mathrm{ps}$ ) on Fig. 4.3(b). In this simulation, the fact that the physical length of the 25 ps pulse (FWHM 5 $\mathrm{mm}$ ) is similar to that of the TFBG was not taken into account (i.e. it was assumed that the electron temperature was the same, as a function of time, over the whole length of the grating). It is still clear from Figs. 4.3(a) and 4.3(b) that during high intensity irradiation by the pumping pulse many resonances shift slightly and some weaken considerably as well, over a certain bandwidth around the SPP peak attenuation, and mostly on the long wavelength side. The broadening of the SPP-induced attenuation agrees with previous observations in free-space plasmonics $[46,47]$. The post-pump recovery is somewhat slower than the rise time because the $25 \mathrm{ps}$ input pulse is much longer than the intrinsic hot carrier relaxation time (less than $1 \mathrm{ps)}$ [22] and because thermalization of excited electrons also occurs through other, slower processes and continue to disrupt the permittivity of the $\mathrm{Au}$. Another factor that will slow down recovery in actual experiments is the temperature dependence of overall refractive index of the structure and the TFBG itself while heat generated by the excitation process must be dissipated. The main prediction of this simulation remains however that the arrival of the pump pulse and its coupling to the hybridized-cladding mode identified in Fig. 4.3(c) will cause a shift in the resonance position of another cladding mode (at the "probe" wavelength in Fig. 4.3(c)). Therefore, a co-propagating continuous wave core mode at the probe wavelength will be modulated synchronously by the pump pulse, but with a slightly longer "tail" (leading to a modulated pulse length of $56 \mathrm{ps}$ ) due to the slow relaxation processes predicted in the two-temperature model Fig. 4.3(d) shows a typical experimental result for the modulated CW probe 
(described in detail in the following section) which agrees well with the predicted pulse shape.

\subsubsection{Ultrafast switching experimental results}
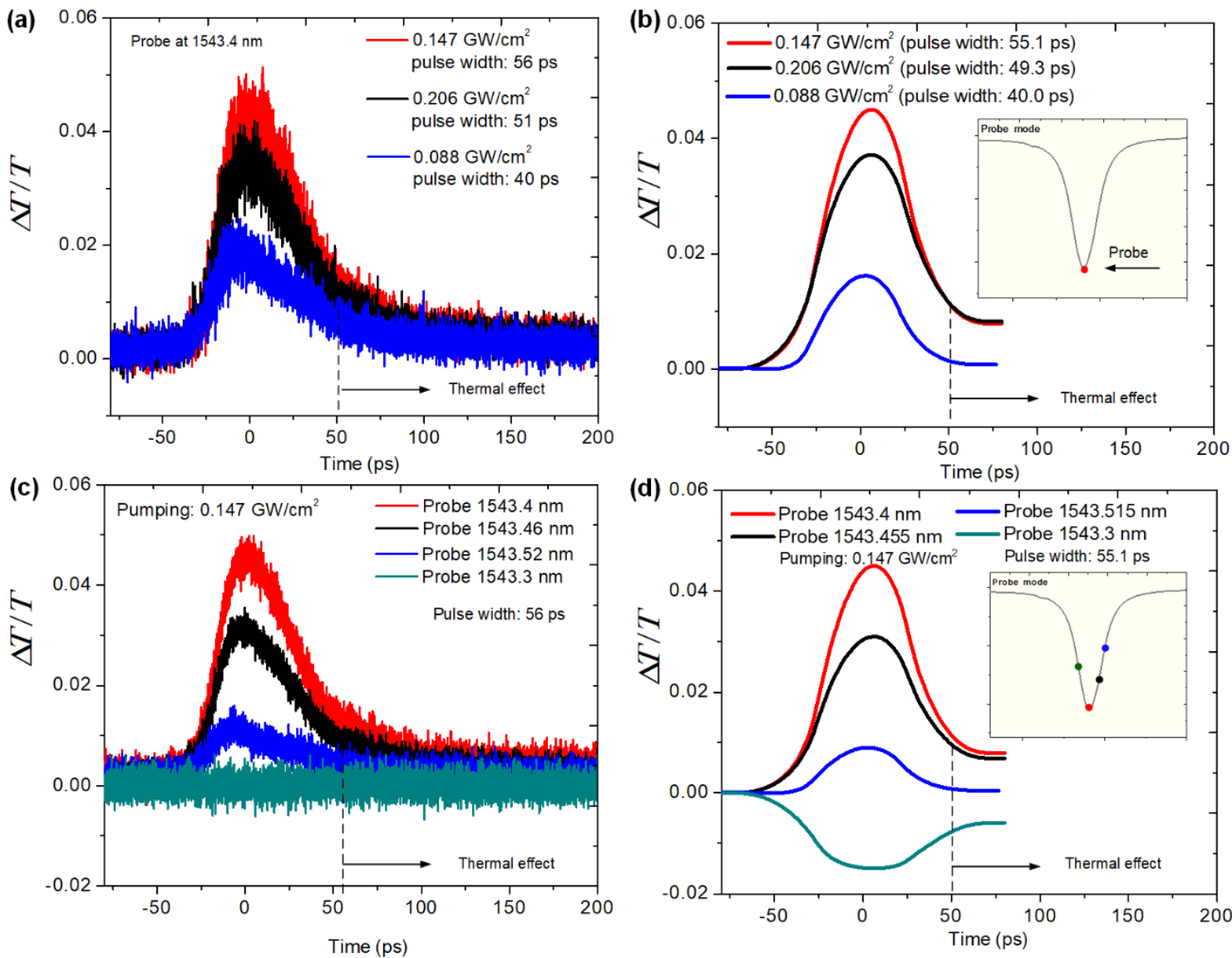

Fig. 4.4. Pump-induced modulation of $\mathrm{CW}$ probe: (a) the output response with pumping power; (b) the simulation for (a); (c) the output response with probe position; (d) the simulation for (c).

Fig. 4.4 shows the change in the transmitted power of a $\mathrm{CW}$ laser probe co-propagating in the fiber core with the pump, as a function of pump power and probe wavelength. The pump wavelength was fixed at $1540.4 \mathrm{~nm}$, on the long wavelength side of the first resonance located on the short wavelength side of the SPP maximum. This pump wavelength was determined experimentally to yield the strongest probe modulation. When 
the probe wavelength is located on the transmission minimum of the resonance, the arrival of the pump pulse blue-shifts the resonance and the transmitted power increases. Fig. 4.4(a) shows the result for this situation, i.e. a pulsed increase of the probe power with rise times ranging from 27 to $39 \mathrm{ps}$ and slightly longer recovery times. The maximum modulation depth increases with pump power and reaches $4.5 \%$ at an average pump power of $50 \mathrm{~mW}$ (corresponding to $.147 \mathrm{GW} / \mathrm{cm}^{2}$ in the metal layer, given an excitation efficiency of $0.7 \%$ between the input power and the guided power in the metal, as described in Supplementary note 8 ). The modulation depth was determined by comparing the power level changes to those measured by blocking the $\mathrm{CW}$ beam. For higher pump powers, the modulation depth of $\mathrm{CW}$ beam decreases, likely due to saturation of $\mathrm{SPR}^{48}$ together with competing slow processes caused by the relatively high $(1 \mathrm{MHz})$ pulse repetition rate of our tunable $25 \mathrm{ps}$ laser [49]. Self-detuning of the pump from its resonance may also contribute to lower efficiencies at higher pump levels. The self-detuning can be observed in simulations (Fig. 4.3(c)) and experiments: the transmitted pumping pulses are broadened (even broken up into a train of smaller pulse) and show an ultrafast amplitude modulation (greater than 35\%) (supplementary note 4, Figs. 4.10 and 4.11). It was verified that the decrease at higher pump powers is not due to damage (such as delamination of the gold film) because it was verified that high modulation efficiencies return after testing at higher pumping levels. Fig. 4.4(b) shows corresponding simulations for transmission at the probe wavelength where the range of power density in the metal layer is from 0.088 to $0.206 \mathrm{GW} / \mathrm{cm}^{2}$.

In Fig. 4.4(c), the switching response at the optimum pump power is measured for different wavelength positions of the probe relative to the resonance. The response time remains stable and the highest efficiency occurs when the probe is right on the resonance 
transmission minimum, in good agreement with simulations (Fig. 4.4(d)), except for the case where the $\mathrm{CW}$ probe is tuned to the shorter wavelength edge of the probe-mode resonance, because the "reverse" pulse generated by the switching is below the background noise.

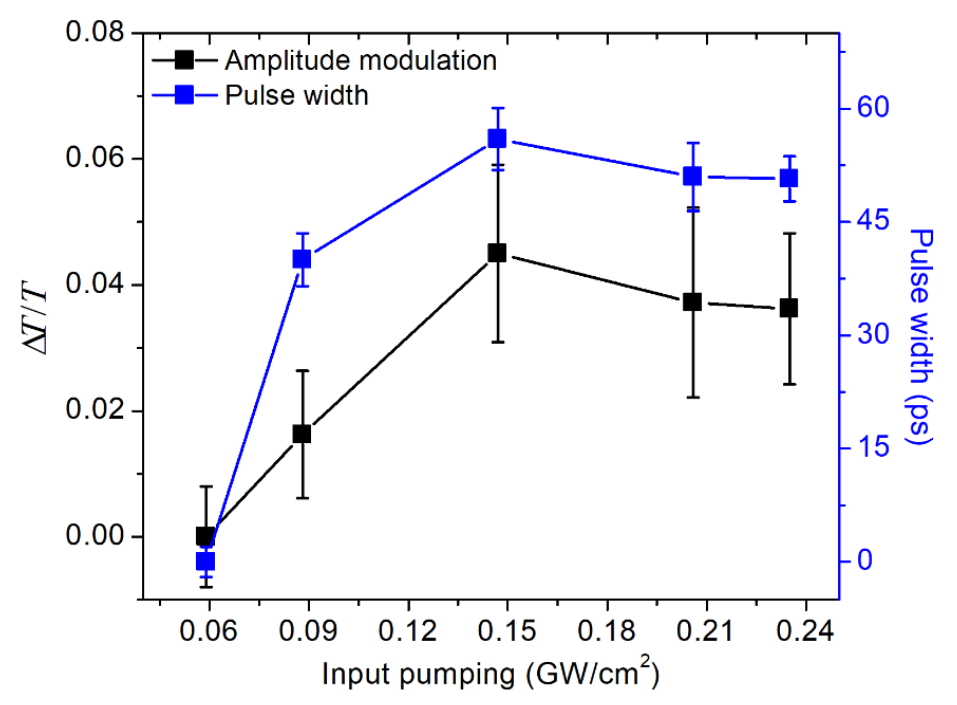

Fig. 4.5. Summary of pump-induced modulation of a CW probe as a function of the power density in the metal layer. The input (pump) pulse width is $25 \mathrm{ps}$.

Fig. 4.5 summarizes the switching results in terms of modulation efficiency and pulse width of the modulated probe, up to the maximum power level available from our system. The maximum efficiency occurs at the same power level as the largest temporal broadening and the effects appear to decrease and saturate beyond that point. Finally, it was verified that no modulation is observed when pumping and probing at resonances further away from the SPP spectral peak, but also at any wavelength (even close to the SPP maximu), for Spolarized input light in agreement with the well-established result that surface plasmon resonances are never observed for gold thicknesses of a few tens of nm due to the fact that this polarization couples only to TE-like cladding modes [23-33]. 


\subsection{Discussion}

The observations reported in chapter 4 constitute both experimental and simulation evidence that hot carriers can be generated by SPP waves excited on the surface of metal coated optical fibers by in-core TFBGs and that these hot carriers modulate the complex permittivity of the metal layer on a picosecond time scale. The spectral width over which the SPP excitation is possible for an optimal gold thickness and low roughness is still quite larger than the wavelength spacing between the grating resonances of the modes of the fiber cladding which means that pumping and probing of the complex permittivity can be carried out simultaneously with different wavelengths co-propagating simultaneously in the single mode core. While the results for high speed modulation of a CW probe by hot carriers from pump pulses show a reasonable overall agreement with simulations based on a very simple model of the light matter interaction, several factors may impact the maximum modulating efficiency observed here. First, the impact of the hot carriers on the complex permittivity not only shifts the transmission resonance at the probe wavelength but also at the pump wavelength which causes a kind of self-modulation of the pump, i.e. the pump pulse "detunes" itself from resonance during the pulse. The main effect of this is an unresolved ultrafast relative intensity noise as large as $+/-18 \%$ superimposed on the measured transmission of the pump pulses which, most significantly, is maximum for the same input pulse intensity where the maximum probe modulation is observed $(0.147$ $\mathrm{GW} / \mathrm{cm}^{2}$ ). Another issue is that the spatial extent of the $25 \mathrm{ps}$ pump pulse in the fiber is around $5 \mathrm{~mm}$, i.e. $1 / 4$ of the grating length. Therefore, contrary to what is assumed in the simulations, an SPP wave is only excited in a small part of the grating at any given instant. While the SPP waves propagate some distance backward from their excitation point and 
the cladding mode responsible for it has a power distribution that falls exponentially with distance from the beginning to the end of the grating (with an effective length of $6 \mathrm{~mm}$, for the pumping resonance, based on coupled-mode theory for contra-directional coupling), it is fair to say that the complex permittivity change associated with the hot carriers is likely to be quite non uniform along the grating, resulting in a fast chirp of the grating resonances. Compounding this issue is the fact that the spectral width of the 25 ps pulses is near 0.13 $\mathrm{nm}$, i.e. roughly the same width as the transmission resonances so that the total power transmission factor results from the convolution of the pulse and resonance spectra. Finally, there may be saturation of the surface plasmon in our layers beyond the maximum switching power ${ }^{48}$ and the efficiency of probe modulation goes down. A significantly more complex theoretical modelling will be required to fully explain these results and lead to improved efficiency by optimizing all the adjustable parameters (such as grating strength and length, and coating material).

\subsection{Conclusion}

In spite of these mitigating factors, an "all single-mode fiber" configuration was demonstrated where core-pumping a gold-coated TFBG in water with 25 ps pulses of near infrared light at $1 \mathrm{MHZ}$ and $50 \mathrm{~mW}$ of average input power resulted in a synchronous modulation of a $\mathrm{CW}$ signal propagating in the core of the same fiber by $4.5 \%$, with a modulating efficiency of $30 \pm 7 \%$ per $\mathrm{GW} / \mathrm{cm}^{2}$ and pulse duration widening of $\sim 30 \mathrm{ps}$. This achievement stems from many factors, including the low insertion of loss of the TFBG inscribed in standard telecom fiber, the dense comb of narrowband cladding mode resonances with high Q factor, and the strong light-matter interaction efficiency of the plasmonic hybridized cladding modes. Even though the reported modulation efficiency 
obtained in these devices is currently too low for conventional switching applications (in data transmission for instance), the fact that watt level input powers can be guided in this kind of fiber without damage leads to further potential applications in fiber-based nonlinear light-matter interactions like nonlinear sensing [50], plasmonic-assisted frequency conversion [51,52], and THz antenna [53]. It is also believed that further experiments with parameter variations (of grating properties, material choices for the coating, and pump

pulse widths for instance) will lead to improved understanding of the effects observed and optimization of their performance.

\subsection{References}

1. W. L. Barnes, A. Dereux, and T. W. Ebbesen, "Surface plasmon subwavelength optics," Nature 424(14), 824-830 (2003).

2. C. Frischkorn and M. Wolf, Chem. "Femtochemistry at metal surface," Chem. Rev. 106, 4207-4233 (2006).

3. M. L. Brongersma, N. J. Halas, and P. Nordlander, "Plasmon-induced hot carrier science and technology," Nat. Nanotechnol. 10, 25-34 (2015).

4. P. Narang, R. Sundararaman, and H. A. Atwater, "Plasmonic hot carrier dynamics in solid-state and chemical systems for energy conversion," Nanophotonics 5(1), 96-111 (2016).

5. R. Lopez, T. E. Haynes, and L. A. Boatner, "Temperature-controlled surface plasmon resonance in $\mathrm{VO}_{2}$ nanorods," Opt. Lett. 27(15), 1327-1329 (2002).

6. T. Jostmeier, M. Mangold, J. Zimmer, H. Karl, H. J. Krenner, C. Ruppert, and M. Betz, "Thermochromic modulation of surface plasmon polaritons in vanadium dioxide nanocomposites," Opt. Express 24(15), 17321-17331 (2016). 
7. R. A. Pala, K. T. Shimizu, N. A. Melosh, and M. L. Brongersma, "Nonvolatile Plasmonic Switch Employing Photochromic Molecules," Nano Lett. 8(5), 1506-1510 (2008).

8. A. Marini, M. Conforti, G. D. Valle, H. W. Lee, T. X. Tran, W. Chang, M. A. Schmidt, S. Longhi, P. St, J. Russell, and F. Biancalana, "Ultrafast nonlinear dynamics of surface plasmon polaritons in gold nanowires due to the intrinsic nonlinearity of metal," New J. Phys. 15, 013033 (2013).

9. R. W. Boyd, Zh. M. Shi, and I. D. Leon, "The third-order nonlinear optical susceptibility of gold," Opt. Commun. 326, 74-79 (2014).

10. I. D. Leon, Zh. M. Shi, A. C. Liapis, and R. W. Boyd, "Measurement of the complex nonlinear optical response of a surface plasmon-polariton," Opt. Lett. 39(8), 2274-2276 (2014).

11. I. D. Leon, J. E. Sipe, and R. W. Boyd, "Self-phase-modulation of surface plasmon polaritons," Phys. Rev. A 89, 013855 (2014).

12. A. Baron, S. Larouche, D. J. Gauthier, and D. R. Smith, "Scaling of the nonlinear response of the surface plasmon polariton at the metal/dielectric interface," J. Opt. Soc. Am. B 32(1), 9-14 (2015).

13. R. H. M. Groeneveld, R. Sprik, and A. Lagendijk, "Ultrafast relaxation of electrons probed by surface plasmons at a thin silver film," Phys. Rev. Lett. 64(7), 784-787 (1990).

14. K. F. MacDonald, Z. L. Samson, M. I. Stockman, and N. I. Zheludev, "Ultrafast active plasmonics," Nat. Photon. 3, 55-58 (2008).

15. N. Rotenberg, J. N. Caspers, and H. M. V. Driel, "Tunable ultrafast control of plasmonic coupling to gold films," Phys. Rev B 80, 245420 (2009). 
16. T. Tyborski, S. Kalusniak, S. Sadofev, F. Henneberger, M. Woerner, and T. Elsaesser, "Ultrafast nonlinear response of bulk plasmons in highly doped $\mathrm{ZnO}$ layers," Phys. Rev. Lett. 115, $147401(2015)$.

17. H. Harutyunyan, A. B. F. Martinson, D. Rosenmann, L. K. Khorashad, L. V. Besteiro, A. O. Govorov, and G. P. Wiederrecht, "Anomalous ultrafast dynamics of hot plasmonic electrons in nanostructures with hot spots," Nat. Nanotechnol. 10, 770-774 (2015).

18. M. Z. Alam, I. D. Leon, and R. W. Boyd, "Large optical nonlinearity of indium tin oxide in its epsilon-near-zero region," Science 352, 795-797 (2016).

19. Y. M. Yang, K. Kelley, E. Sachet, S. Campione, T. S. Luk, J. P. Maria, M. B. Sinclair, and I. Brener, "Femtosecond optical polarization switching using a cadmium oxidebased perfect absorber," Nat. Photon. 11, 390-395 (2017).

20. M. Bernardi, J. Mustafa, J. B. Neaton, and S. G. Louie, "Theory and computation of hot carriers generated by surface plasmon polaritons in noble metals," Nat. Commun. 6, 7044 (2015).

21. R. Sundararaman, P. Narang, A. S. Jermyn, W. A. Goddard III, and A. Atwater, "Theoretical predications for hot-carrier generation from surface plasmon decay," Nat. Commun. 5, 5788 (2014).

22. A. M. Brown, R. Sundararaman, P. Narang, W. A. Goddard, and H. A. Atwater, "Nonradiative Plasmon decay and hot carrier dynamics: effects of phonons, surfaces, and geometry," ACS Nano 10, 957-966 (2016).

23. J. Albert, L. Y. Shao, and C. Caucheteur, "Tilted fiber Bragg grating sensor," Laser Photonics Rev. 7(1), 83-108 (2013). 
24. J. Albert, S. Lepinay, C. Caucheteur, and M. C. DeRosa, "High resolution gratingassisted surface plasmon resonance fiber optic aptasensor," Methods 63, 239-254 (2013).

25. C. Caucheteur, T. Guo, and J. Albert, "Review of plasmonic fiber optic biochemical sensors: improving the limit of detection," Anal. Bioanal. Chem. 407, 3883-3897 (2015).

26. Y. Shevchenko, T. J. Francis, D. A. D. Blair, R. Walsh, M. C. DeRosa, and J. Albert, "In site biosensing with a surface plasmon resonance fiber grating aptasensor," Anal. Chem. 83, 7027-7034 (2011).

27. V. Voisin, J. Pilate, P. Damman, P. Megret, and C. Caucheteur, "Highly sensitive detection of molecular interactions with plasmonic optical fiber grating sensors," Biosens. Bioelectron. 51, 249-254 (2014).

28. T. Guo, F. Liu, X. Liang, X. H. Qiu, Y. Y. Huang, Ch. Xie, P. Xu, W. Mao, B. O. Guan, and J. Albert, "Highly sensitive detection of urinary protein variations using tilted fiber grating sensors with plasmonic nanocoatings," Biosens. Bioelectron. 78, 221-228 (2016). 29. Y. Yuan, T. Guo, X. H. Qiu, J. H. Tang, Y. Y. Huang, L. Zhuang, Sh. G. Zhou, Zh. H. Li, B. O. Guan, X. M. Zhang, and J. Albert, "Electrochemical surface plasmon resonance fiber-optic sensor: In situ detection of electroactive biofilms," Anal. Chem. 88, 76097616 (2016).

30. Z. C. Zhang, T. Guo, X. J. Zhang, J. Xu, W. P. Xie, M. Nie, Q. Wu, B. O. Guan, and J. Albert, "Plasmonic fiber-optic vector magnetometer," Appl. Phys. Lett. 108, 101105 (2016).

31. C. Caucheteur, T. Guo, F. Liu, B. O. Guan, and J. Albert, "Ultrasensitive plasmonic sensing in air using optical fibre spectral combs," Nat. Commun. 7, 13371 (2016). 
32. X. J. Zhang, Sh. Sh. Cai, F. Liu, H. Chen, P. G. Yan, Y. Yuan, T. Guo, and J. Albert, "In situ determination of the complex permittivity of ultrathin $\mathrm{H} 2$-infused palladium coatings for plasmonic fiber optic sensors in the near infrared," J. Mater. Chem. C 6, 5161-5170 (2018).

33. J. J. Lao, P. Sun, F. Liu, X. J. Zhang, Ch. X. Zhao, W. J. Mai, T. Guo, G. Zh. Xiao, and J. Albert, "In situ plasmonic optical fiber detection of the state of charge of supercapacitors for renewable energy storage," Light Sci. Appl. 7, 34 (2018).

34. P. B. Johnson and R. W. Christy, "Optical constants of the noble metals," Phys. Rev. B 6(12), 4370-4379 (1972).

35. G. M. Hale and M. R. Querry, "Optical constants of water in the 200-nm to 200-um wavelength region," Appl. Opt. 12(3), 555-563 (1973).

36. M. Z. Alam and J. Albert, "Selective excitation of radially and azimuthally polarized optical fiber cladding modes," J. Lightwave Technol. 31(19), 3167-3175 (2013).

37. H. Raether, Surface Plasmons on smooth and rough surface and on Gratings. (Springer Tracts in Modern Physics, 1988).

38. J. B. Khurgin, "Ultimate limit of field confinement by surface plasmon polaritons," Faraday Discuss. 178, 109-122 (2015).

39. E. Carpene, "Ultrafast laser irradiation of metals: Beyond the two-temperature model," Phys. Rev B 74, 024301 (2006).

40. S. D. Brorson, J. G. Fujimoto, and E. P. Ippen, "Femtosecond electronic heat-transport dynamics in thin gold films," Phys. Rev. Lett. 59(17), 1962-1965 (1987). 
41. C. K. Sun, F. Vallee, L. H. Acioli, E. P. Ippen, and J. G. Fujimoto, "Femtosecondtunable measurement of electron thermalization in gold," Phys. Rev B 50(20), 1533715348 (1994).

42. R. H. M. Groeneveld, R. Sprik, "Femtosecond spectroscopy of electron-electron and electron-phonon energy relaxation in Ag and Au," Phys. Rev B 51(17), 11433-11445 (1995).

43. J. Renger, R. Quidant, N. V. Hulst, S. Palomba, and L. Novotny, "Free-space excitation of propagating surface plasmon polaritons by nonlinear four-wave mixing," Phys. Rev. Lett. 103, 266802 (2009).

44. A. O. Govorov, H. Zhang, H. V. Demir, and Y. K. Gun'ko, "Photogeneration of hot plasmonic electrons with metal nanocrystals: Quantum description and potential application," Nano Today 9, 85-101 (2014).

45. A. O. Govorov, H. Zhang, "Kinetic density functional theory for plasmonic nanostructures: generation of hot electrons," J. Phys. Chem. C 119, 6181-6194 (2015).

46. S. Park, M. Pelton, M. Zh. Liu, P. Guyot-Sionnest, and N. F. Scherer, "Ultrafast resonant dynamics of surface plasmons in gold nanorods," J. Phys. Chem. C 111, 116123 (2007).

47. M. Perner, P. Bost, U. Lenner, G. V. Plessen, and J. Feldmann, "Optically induced damping of the surface plasmon resonance in gold colloids," Phys. Rev. Lett. 78(11), 2192-2195 (1997).

48. K. H. Kim, A. Husakou, and J. Herrmann, "Saturable absorption in composites doped with metal nanoparticles," Opt. Express 18(21), 21918-21925 (2010). 
49. M. V. Exter, A. Lagendijk, "Ultrashort surface-plasmon and phonon dynamics," Phys. Rev. Lett. 60(1), 49-52 (1988).

50. M. Mesch, B. Metzger, M. Hentschel and H. Giessen, "Nonlinear plasmonic sensing," Nano Lett. 16, 3155-3159 (2016).

51. H. J. Simon, D. E. Mitchell, and J. G. Watson, "Optical second-harmonic generation with surface plasmons in silver films," Phys. Rev. Lett. 33(26), 1531-1534 (1974).

52. T. Y. F. Tsang, "Surface-plasmon-enhanced third-harmonic generation in thin silver films," Opt. Lett. 21(4), 245-247 (1996).

53. L. Novotny, N. V. Hulst, "Antennas for light," Nat. Photon. 5, 83-90 (2011). 


\subsection{Supplementary information}

\subsubsection{Supplementary note 1: Physical characterization of Au layer}

The uniform metal coating process can be controlled properly with the thermal evaporation method. Post-deposition verification by AFM microscopy in Fig. 4.6 shows a thickness of $44 \mathrm{~nm}$ and a roughness $0.87 \mathrm{~nm}$ ). The small roughness ensures low parasitic loss in the metal layer from contributions other than the excitation of a surface plasmon wave, such as scattering and localized SPR. An SEM image is also shown to described the larger scale homogeneity of the coating on the fibre.

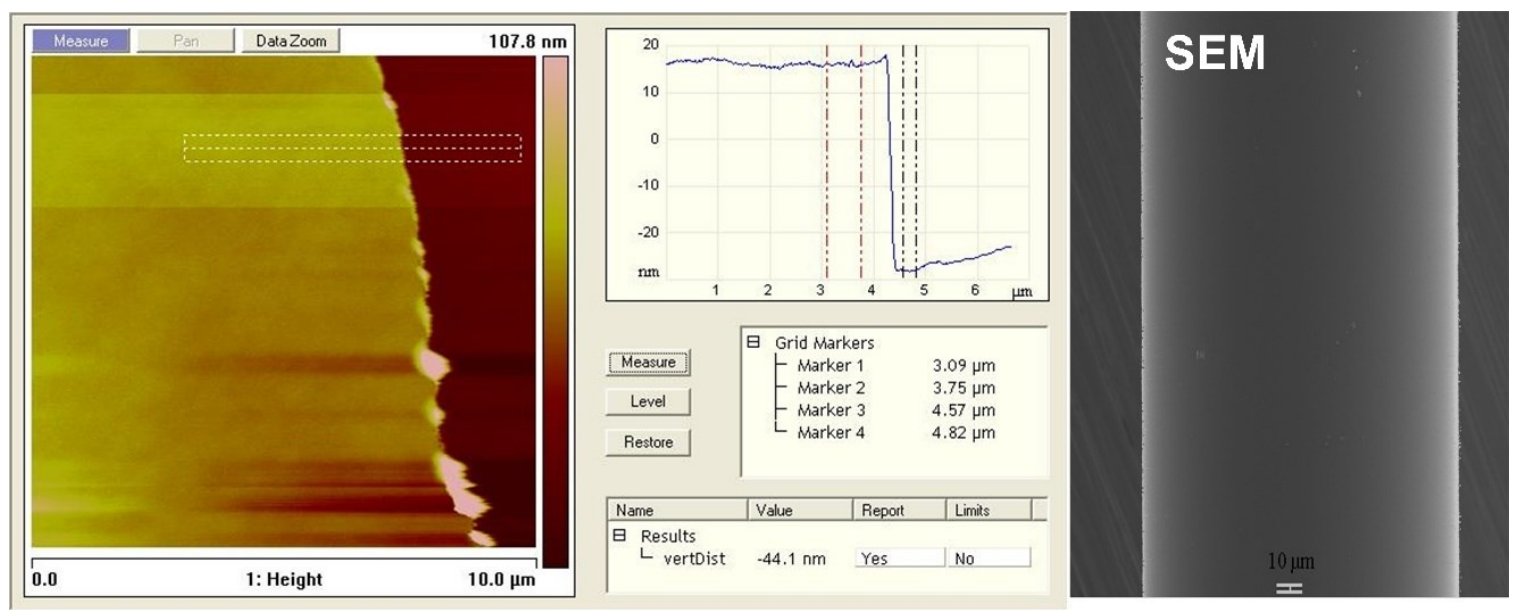

Fig. 4.6. AFM and SEM images of the Au coating on the fiber cladding.

\subsubsection{Supplementary note 2: Delayed two-temperature model}

We used the delayed two-temperature model to simulate the temperature of the hot electrons and lattice. We neglect the lattice contribution to the thermal diffusion and the heat diffusion by hot electrons in the axial direction. With pumping, the generated hot electrons acquire a non-thermal energy distribution. The evolution of the electrons and lattice temperature of the metal after absorption of a laser pulse near a surface plasmon resonance can be expressed as [S1-S3]: 


$$
\begin{aligned}
& C_{e} \frac{\partial T_{e}(t)}{\partial t}=-g_{e p}\left(T_{e}(t)-T_{l}(t)\right)+\frac{N(t)}{2 \tau_{e e}(t)} \\
& C_{l} \frac{\partial T_{l}(t)}{\partial t}=g_{e p}\left(T_{e}(t)-T_{l}(t)\right)+\frac{N(t)}{\tau_{e p}(t)} \\
& \frac{\partial N(t)}{\partial t}=-\frac{N(t)}{2 \tau_{e e}(t)}-\frac{N(t)}{\tau_{e p}(t)}+P(z, t)
\end{aligned}
$$

where $C_{e}$ and $C_{l}$ are the electronic and lattice heat capacities $\left(C_{l}=2.5 \times 10^{6} \mathrm{~J} \cdot \mathrm{m}^{-3} \cdot \mathrm{K}^{-1}\right.$ and $C_{e}=\gamma T_{e}(t)$ where $\gamma=71 \mathrm{~J} \cdot \mathrm{m}^{-3} \cdot \mathrm{K}^{-2}$ for gold $), g_{e p}=2.3 \times 10^{16} \mathrm{~W} \cdot \mathrm{m}^{-3} \cdot \mathrm{K}^{-1}$ is the electronphonon coupling coefficient of gold $[\mathrm{S} 2], T_{e}\left(T_{l}\right)$ is the temperature of the thermal electrons (lattice), we assume a Gaussian pump temporal dependence $P(t)=(1-R-T) \alpha e^{-a z} P_{0} \exp \left(-2\left(t / \tau_{p}\right)\right), \tau_{p}$ is the pulse duration, $\mathrm{R}$ is the reflection, $\mathrm{T}$ is the transmission, $\alpha$ is the absorption coefficient of the pulse at the SPR, The relaxation time of electron-electron collision $\left(\tau_{e e}\right)$ and electron-phonon collision $\left(\tau_{e p}\right)$ can be expressed as:

$$
\begin{aligned}
\tau_{e e} & =\left[\frac{\omega^{2}}{4 \pi^{2} \omega_{p}}\left[1+\left(\frac{2 \pi k_{B} T_{e}}{\hbar \omega}\right)^{2}\right]\right]^{-1} \\
\tau_{e p} & =\frac{\gamma\left(T_{e}+T_{l}\right)}{2 g_{e p}}
\end{aligned}
$$

Fig. 4.7 shows the electron and lattice dynamics upon pumping by a 25 ps pulse. Since the thermal effect is easily induced from phonon-phonon scattering to the lattice, as can be seen from the tail of the temperature rise at long durations, a relatively weak pumping is more suitable for the observation of ultrafast switching. 


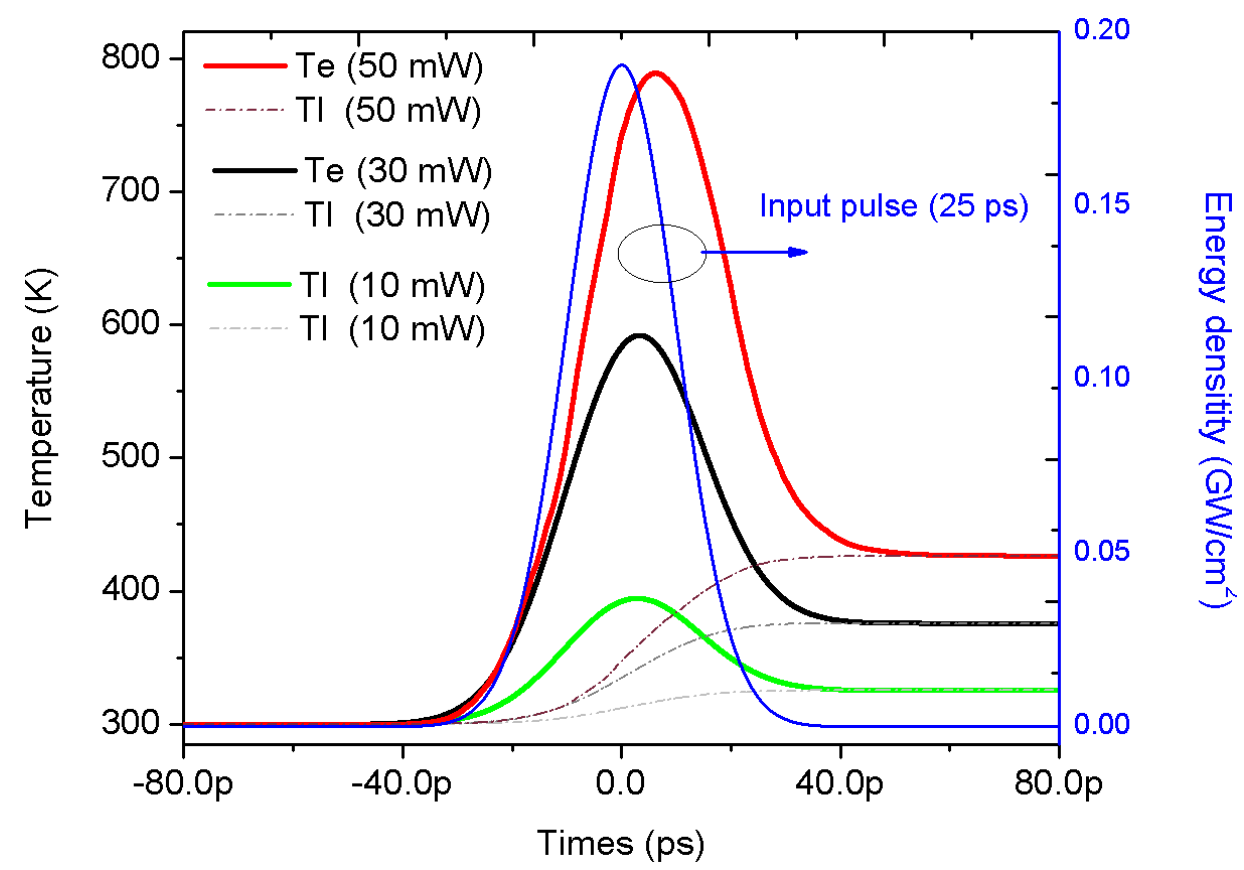

Fig. 4.7. The transient response of $T_{e}$ and $T_{1}$ for hot carriers.

\subsubsection{Supplementary note 3: The dynamics of the dielectric function of the Au layer}

\section{based on hot carrier relaxation}

As for the dielectric function of a thin gold film, we are using the Drude model with two critical points (DCP) [S4,S5].

$$
\varepsilon(\omega)=\varepsilon_{\infty}-\frac{\varepsilon_{p}^{2}}{\omega^{2}+i \Gamma_{A u} \omega}+\sum_{i=1}^{2} C_{i} E_{i}\left(\frac{e^{i \Phi}}{E_{i}-\omega-i \gamma}+\frac{e^{-i \Phi}}{E_{i}+\omega+i \gamma}\right)
$$

where $\varepsilon_{\infty}, \omega_{p}$ and $\Gamma_{A u}$ are the background dielectric constant, plasma frequency and linewidth; $C_{i}, E_{i}, \gamma$ and $\Phi_{i}$ are the oscillator strength, oscillator energy, oscillator damping and oscillator phase, respectively.

The influence of pumping on the effective mass $m_{\text {eff }}$ of the electron and on the carrier density $n_{e}$ can be neglected in the plasma frequency $\omega_{p}=\sqrt{n_{e} e^{2} / m_{e f} \varepsilon_{0}}$ for switching [S6] because the hot carriers excited by surface plasmon decay at intraband transition close to 
$1550 \mathrm{~nm}$ is too far away from the plasma frequency $(\sim 139 \mathrm{~nm})$ and interband transition $(\sim 520 \mathrm{~nm})$. Therefore, the Drude damping term $\Gamma_{A u}=\frac{\hbar}{\tau}$ will dominate the switching process, where $\tau$ is the electron relaxation time:

$$
\frac{1}{\tau}=\frac{1}{\tau_{e e}}+\frac{1}{\tau_{e p}}
$$

According to Equation 4.5, the electron-electron scattering rate $1 / \tau_{e e}$ and electronphonon scattering rate $1 / \tau_{e p}$ are related to the time-dependent electron and phonon temperatures which modulate the gold dispersion at the intraband transition near 1540.4 $\mathrm{nm}$. The imaginary part of the dielectric function is strongly affected, with a corresponding effect on the RI of $\mathrm{Au}$ (45 nm thick) as shown in Fig. 4.8.
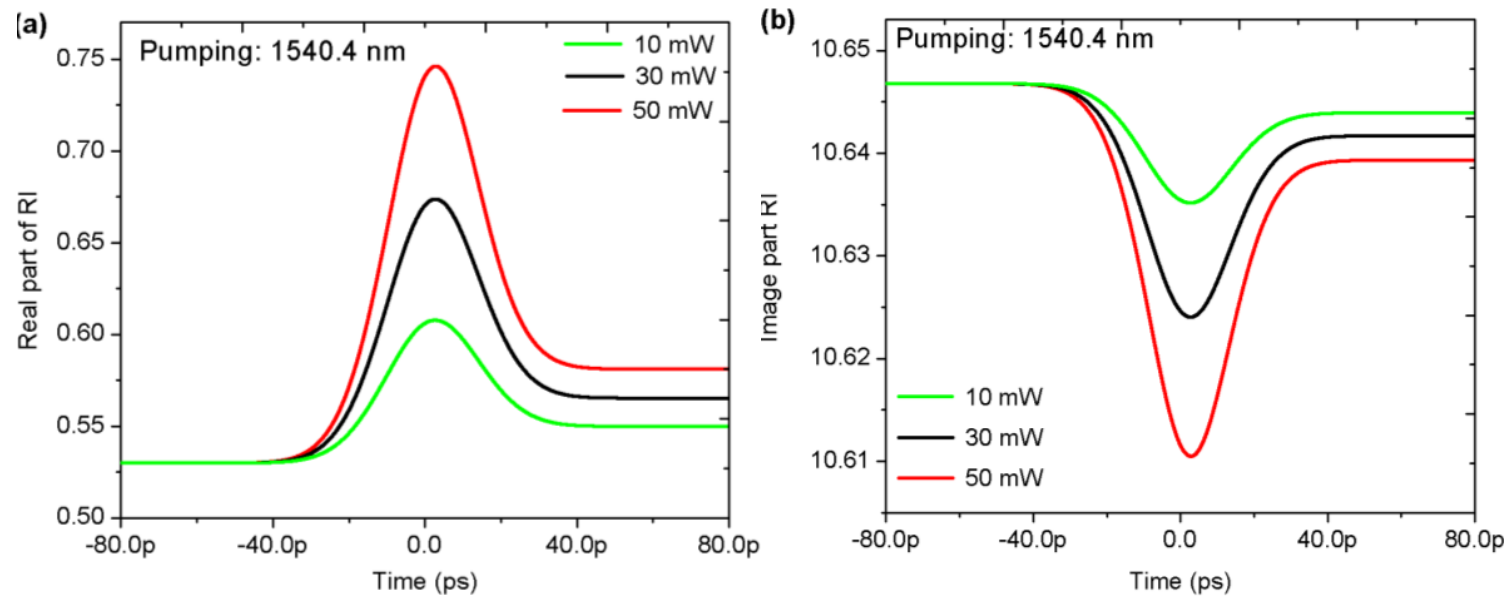

Fig. 4.8. Evolution of the (a) real and (b) imaginary part of effective RI of the Au film 


\subsubsection{Supplementary note 4: Experiment configuration and pump pulse response}
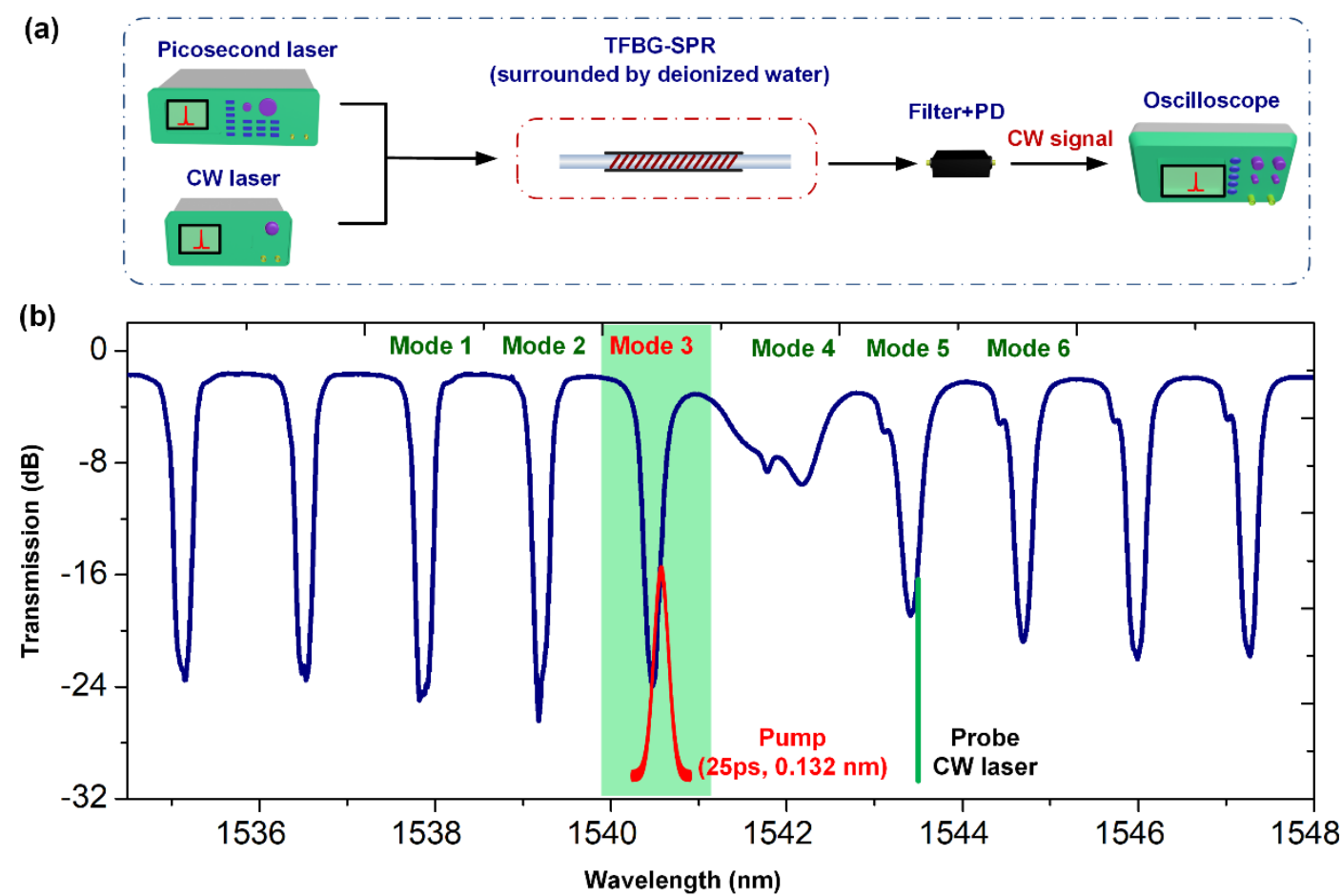

Fig. 4.9. (a) Schematic diagram of the switching system using a New Focus $40 \mathrm{GHz}$ bandwidth photodetector ("PD") and a Yenista tunable band blocking filter ("filter") ( (for measurement of the pump pulse variations, the pump filter is removed); (b) Measured transmission spectrum of the TFBG-SPR

The experimental configuration is shown in Fig. 4.9(a) while Fig. 4.9(b) shows the experimental transmission spectrum around the SPR with a simulated pump pulse spectrum. Fig. S5 shows the photodetector response measurement of the input and output pump pulses at different pump wavelengths (aligned with different cladding modes). The pump pulse temporal broadening is largest when lined up with mode 4, i.e. at the peak of the SPR and larger for modes 2 and 3 than for modes 5 and 6 since the photon energy at modes 2 and 3 is higher than that of the surface plasmon (i.e., corresponding to the two-level system assumption of SPR [S6]). Furthermore, a strong chaotic amplitude modulation is only 
happening for the pump aligned with mode 3, i.e., on the short wavelength (high energy) side of the SPR, where the plasmonic hybrid guided mode sensing sensitivity is large enough to respond to the hot carrier relaxation changes inside the metal layer.
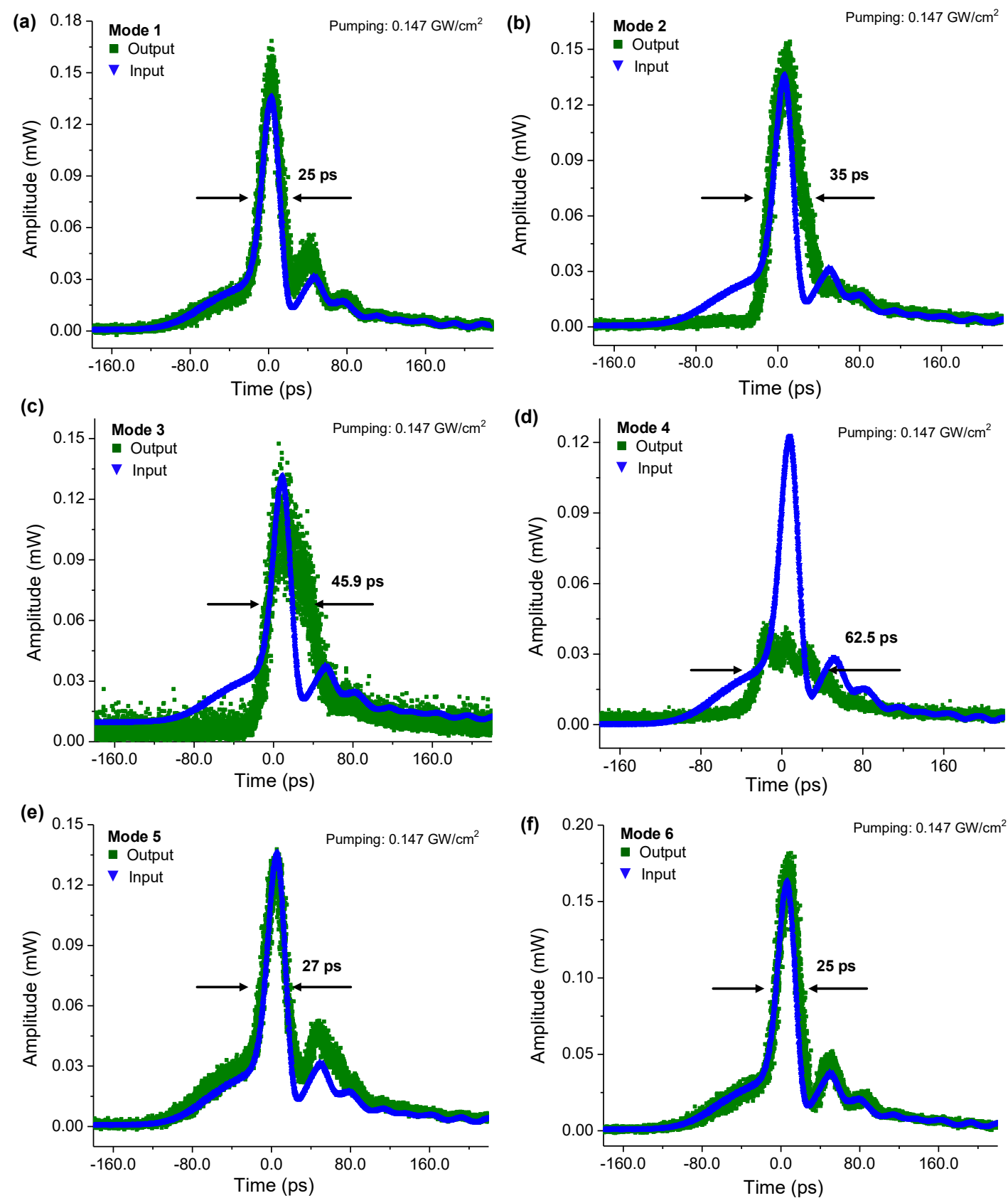

Fig. 4.10. The pump pulse response for pumping at different cladding mode wavelengths (see Fig. 4.9 for wavelengths corresponding to mode numbers). 
Fig. 4.11 shows the ultrafast amplitude modulation efficiency of the pumping pulse when pumping at mode 3 vs pumping power. The peak modulation reaches $36 \%$ at a pumping power of $0.118 \mathrm{GW} / \mathrm{cm}^{2}$ and then decreases gradually with higher pump power.
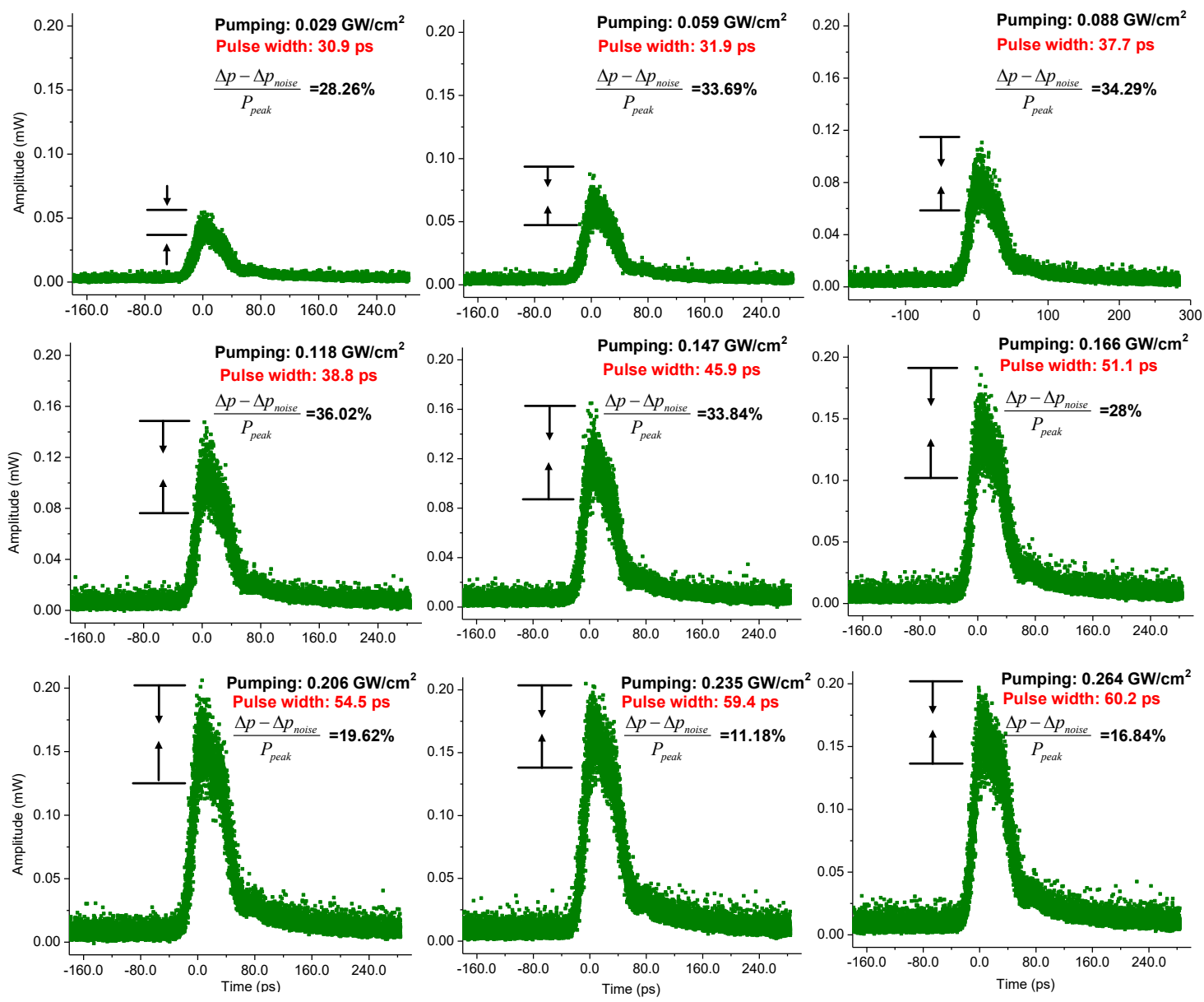

Fig. 4.11. The transmission of pump pulses at mode 3 versus pumping power. 


\subsubsection{Supplementary note 5: Plasmonic guided mode}

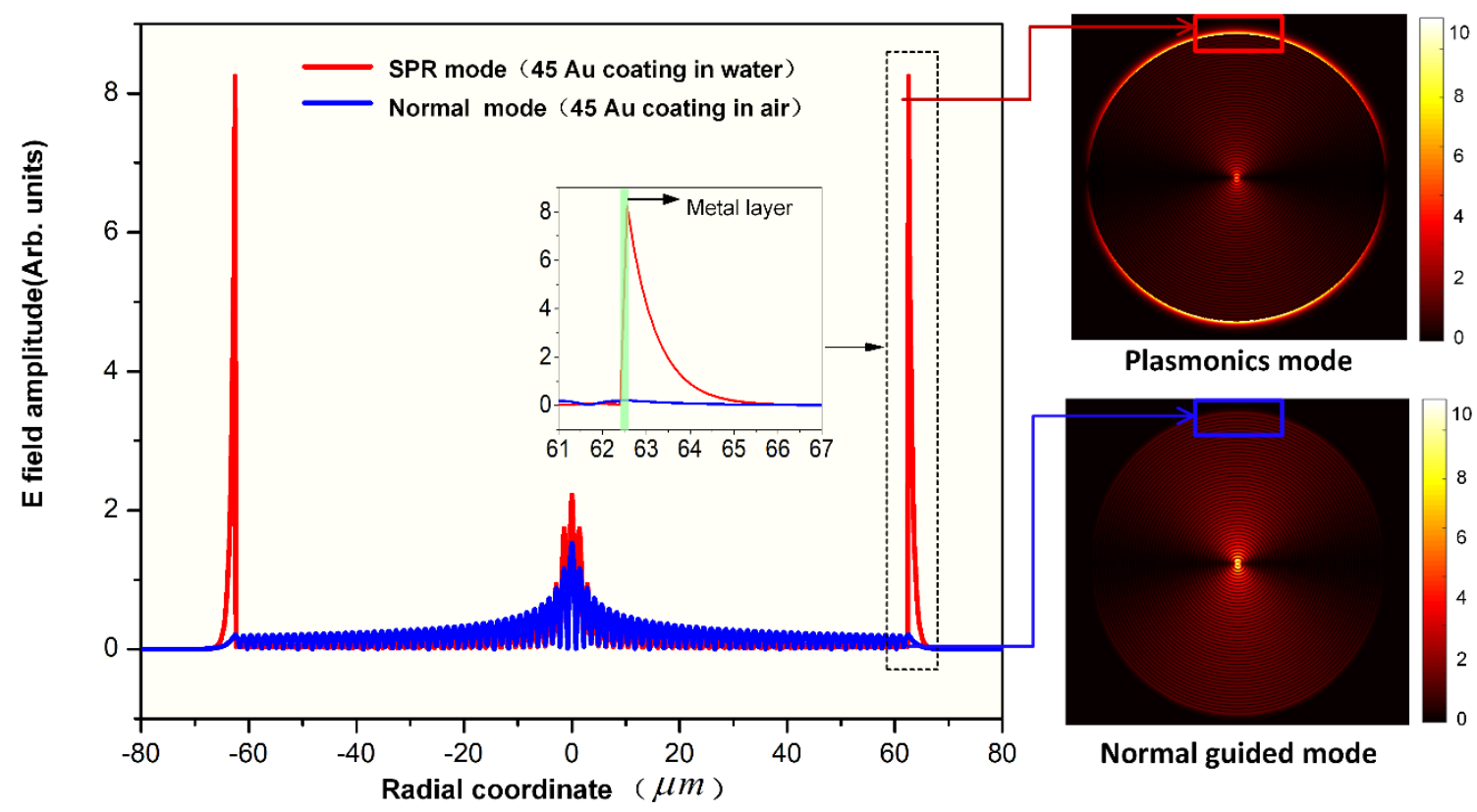

Fig. 4.12. Radial dependence of simulated plasmonic guided mode E-fields versus normal guided mode E-field. Insets show corresponding 2D intensity profiles

Fig. 4.12 shows the radial dependence of the radial electric field vectors and 2D mode intensity distributions of SPR-active and non-active cladding modes of the fiber simulated by the FIMMWAVE mode solver. The SPR significantly enhances the localized energy at the fiber surface and the fill factor of the mode field intensity increases significantly in the $45 \mathrm{~nm}$ thick metal layer, up to $2 \%$, (Fig. 4.12, red curve) while a normal guided mode without plasmonic excitation can only support a maximum of $0.035 \%$ of the cladding mode fill factor in the same layer (Fig. 4.12, blue curve). The mode used for pumping in the reported experiments corresponds to the adjacent resonance to the SPP maximum on the short wavelength side and its fill factor is $0.74 \%$ in the metal. 


\subsubsection{Supplementary note 6: Effective linear susceptibility of the SPR}

In the weak pumping approximation, we consider the TFBG-SPR dispersion properties based on the loss from collective electron collision damping only. The effective linear susceptibility can be described by using the typical Lorentz model and by replacing the atom vibration resonance and damping by the frequency and damping term of the SPR:

$$
\chi_{e f f}(\omega)=\frac{e^{2} N_{e f f}}{m_{e f f} \varepsilon_{0}\left(\omega_{S P R}^{2}-\omega^{2}-2 i \omega \Gamma\right)}
$$

where $N_{\text {eff }}=5.458 \times 10^{23} \mathrm{~cm}^{-3}$ is the effective carrier density of the SPR, $e=4.8 \times 10^{-10}$ esu is the electron charge, $m_{\text {eff }}$ is the effective mass $\left(0.3 \mathrm{~m}_{\mathrm{o}}\right), \varepsilon_{0}$ is the permittivity in vacuum, $\omega_{S P R}$ is the SPR frequency, $\Gamma$ is the bandwidth of the SPR $(0.32$ nm). Furthermore, the wavelength difference between cladding modes with- and withoutSPR can be simulated by $\Delta \lambda=\sqrt{1+\chi_{\text {eff }}(\omega)} \cdot S_{S P R}$, where $S_{S P R}=589.8 \mathrm{~nm} / R I U$ is the SPR cladding mode sensitivity [S8].

\subsubsection{Supplementary note 7: TFBG-SPR Au-thickness dependence}

Fig. 4.13 shows the TFBG-SPR spectra modulated by thickness of Au layer, simulated by cylindrical finite-difference vector mode solver for modes and coupled mode theory for spectra [S9]. Transmission spectra simulations (with accepted values for bulk Au thin film refractive indices, including wavelength dispersion) [S10] were performed for thicknesses from $10 \mathrm{~nm}$ to $70 \mathrm{~nm}$. The properties were used for fiber as follows: core radius $=4.1 \mathrm{um}$, cladding radius $=62.5 \mathrm{um}$, cladding material of pure silica $(\mathrm{SiO} 2)$, and core material of germanium-doped silica with 0.0625 germanium/silicon ratio [S11]. The "best" SPR response (spectrally narrowest and with the highest localization of cladding mode energy in the metal layer) is obtained for a thickness of $44 \mathrm{~nm}$. 

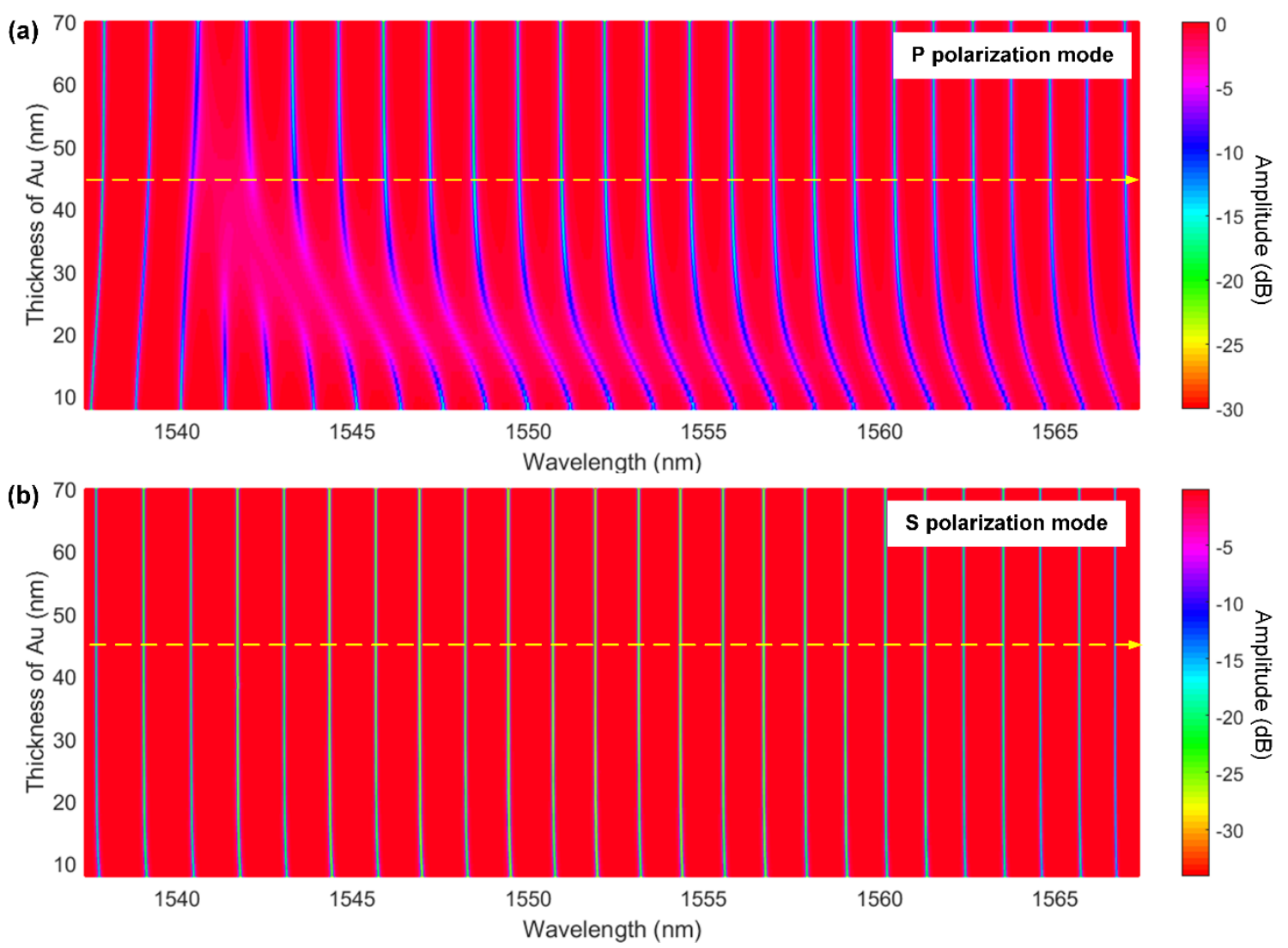

Fig. 4.13. Simulated TFBG-SPR Au thickness dependence: (a) P-polarized input core mode; (b) Spolarized.

\subsubsection{Supplementary note 8: Plasmon excitation efficiency and energy density inside} the metal layer

As for the energy density inside the metal layer: $P_{\text {density }}=\frac{P_{\text {ave }}}{\delta_{p} \cdot H \cdot S} \cdot f_{\text {filling }} \cdot R_{\text {grating }}$, where $P_{\text {ave }}$ is the average power, $\delta_{p}$ is the input pulse width (25 ps), $H$ is the pulse repetition rate (1 $\mathrm{MHz}), S_{\text {metal }}=2 \pi r L_{d}$ is the total surface of the gold layer on the fiber cladding ( $r=62.5 \mu \mathrm{m}$ is the fiber radius, $L_{d}=22.96 \mathrm{~nm}$ is the skin depth of the enhanced plasmonic field in the metal layer $\left.\left(L_{d}=\frac{\lambda}{2 \pi}\left(\frac{\varepsilon_{A u}^{\prime}+\varepsilon_{\text {water }}}{\varepsilon_{A u}^{\prime 2}}\right)^{1 / 2}\right)\right), f=0.00736$ is the fill factor of the SPP 
mode in the metal layer, and $R_{\text {grating }}=0.94$ is the coupling efficiency from the core-guided light to the cladding mode which couples the incident laser power into the metal layer. The coupling efficiency from the input mode power to the power guided in the metal layer is given by the product of $f$ and Rgrating, i.e. 0.007 here.

\subsection{Supplementary references}

S1. N. D. Fatti, C. Voisin, M. Achermann, S. Tzortzakis, D. Christofilos, and F. Vallee, "Nonequilibrium dynamics in noble metals," Phys. Rev B 61(24), 16956-16966 (2000).

S2. E. Carpene, "Ultrafast laser irradiation of metals: Beyond the two-temperature model," Phys. Rev B 74, 024301 (2006).

S3. M. Conforti and G. D. Valle, "Derivation of third-order nonlinear susceptibility of thin metal films as a delayed optical response," Phys. Rev B 85, 245423 (2012).

S4. H. Reddy, U. Guler, A. V. Kildishev, A. Boltasseva, and V. M. Shalaev, "Temperaturedependent optical properties of gold thin films," Opt. Mater. Express 6(9), 2776-2802 (2016).

S5. E. T. Hu, Q. Y. Cai, R. J. Zhang, Y. F. Wei, W. Ch. Zhou, S. Y. Wang, Y. X. Zheng, W. Wei, and L. Y. Chen, "Effective method to study the thickness-dependent dielectric functions of nanometal thin film," Opt. Lett. 41(21), 4907-4910 (2016).

S6. Y. M. Yang, K. Kelley, E. Sachet, S. Campione, T. S. Luk, J. P. Maria, M. B. Sinclair, and I. Brener, "Femtosecond optical polarization switching using a cadmium oxide-based perfect absorber," Nat. Photon. 11, 390-395 (2017).

S7. A. Archambault, F. Marquier, and J. J. Greffet, "Quantum theory of spontaneous and stimulated emission of surface plasmons," Phys. Rev B 82, 035411 (2010). 
S8. D. Y. Feng, W. J. Zhou, X. G. Qiao, and J. Albert, "High resolution fiber optic surface plasmon resonance sensors with single-sided gold coatings," Opt. Express 24(15), 1645616464 (2016).

S9. T. Erdogan and J. E. Sipe, "Tilted fiber phase gratings," J. Opt. Soc. Am. A 13(2), 296313 (1996).

S10. R. L. Olmon, B. Slovick, T. W. Johnson, D. Shelton, S. H. Oh, G. D. Boreman, and M. B. Raschke, "Optical dielectric function of gold," Phys. Rev B 86, 235147 (2012).

S11. W. J. Zhou, Y. Zhou, and J. Albert, "A true fiber optic refractometer," Laser Photonics Rev 11(1), 1600157 (2017). 


\section{Chapter 5: Broadband anti-reflection coatings for fiber cladding modes by partially etched nanoscale silver films at percolation (future work)}

Recently, ultrathin metallic films were demonstrated to work as efficient impedancematching, antireflection coating layers in the terahertz and mid-infrared frequency ranges, showing broadband performance [1-3]. In TFBGs, the tilted grating plane resonantly couples the input light from the forward-propagating core mode to a large number of backward-propagating cladding modes, resulting in a series of perfect "comb"-like discrete resonances over a large wavelength range in transmission, which are sensitive to external coatings such as thin metal films. In this chapter, the thickness and roughness of a $50 \mathrm{~nm}$ thick silver film on the surface of a TFBG was gradually etched by $\mathrm{H}_{2} \mathrm{O}_{2}$ in water while being measured in transmission. A special point, identified by a complete collapse of the resonances (i.e. at the percolation of the Ag film where the metal-insulator phase transition occurs together with tremendous permittivity change (real part) from negative to positive), corresponds to the silver film providing full impedance matching for cladding modes between glass and the water solution at near infrared wavelengths, since the spectrum is then identical to that of a bare TFBG immersed in index matching liquid. 


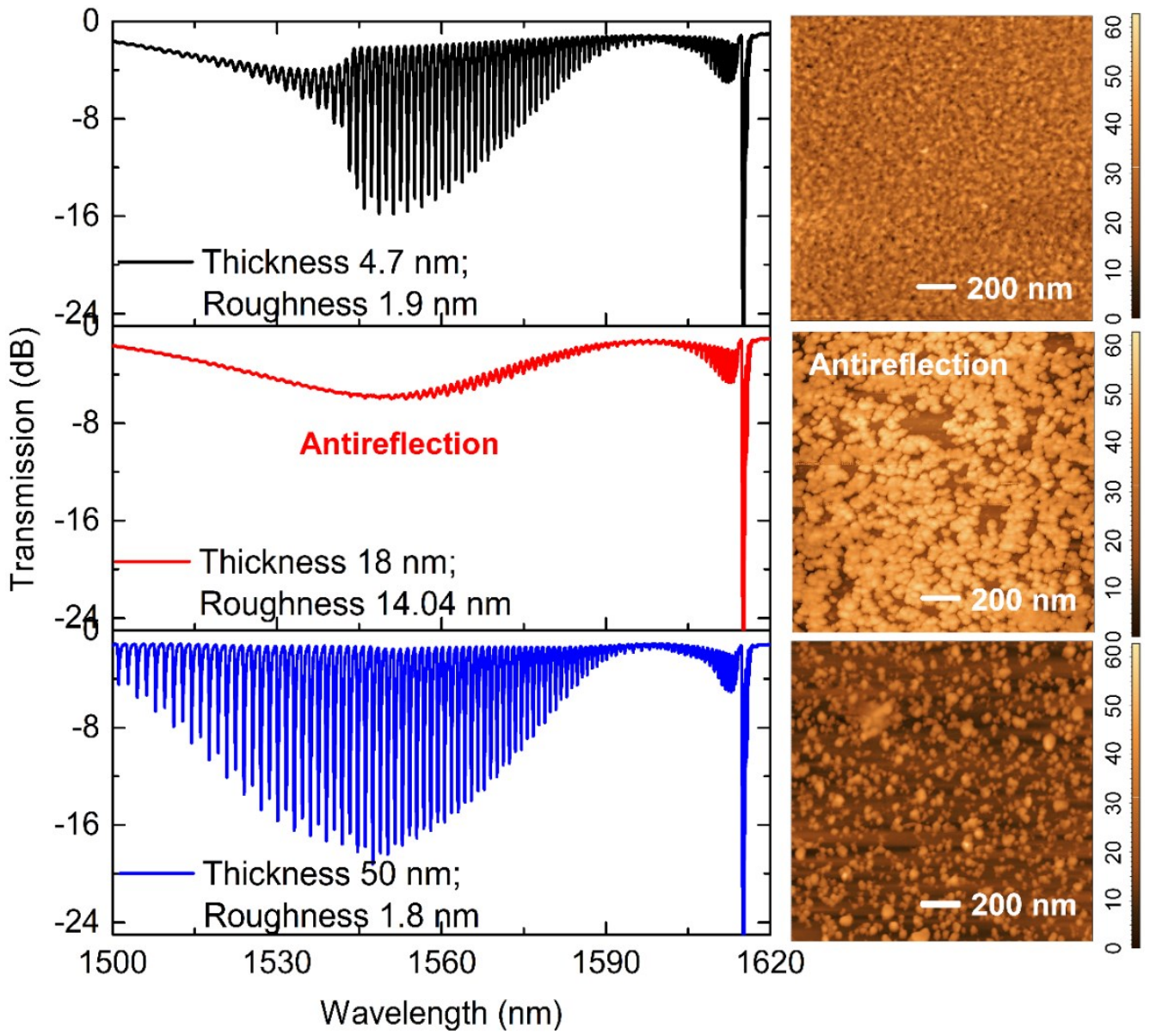

Fig. 5.1. Transmission spectra of a silver coated TFBG with S-polarized input light at three stages of etching of the silver coating (left) and corresponding AFM images of the silver film (right).

In order to detect more details of the metal properties, a $50 \mathrm{~nm}$ thick silver film coated on the surface of a TFBG was gradually etched by $\mathrm{H}_{2} \mathrm{O}_{2}$ in water while being measured in transmission. By controlling the etching speed to a very low value by using a very dilute concentration of liquid etchant $((1: 10,000)$ solutions of Hydrogen Peroxide $(30 \%$ VLSI grade Amplex Chemical Products Ltd) in deionized water), it takes 6 hours to bring the metal layer from its metallic state to its non-metallic state and thousands of spectra can be acquired in real time during a full etch. Fig. 5.1 shows the experimentally measured transmission spectra of the $12^{\circ}$ silver-coated TFBG. The initially smooth $50 \mathrm{~nm}$ thick silver 
film becomes thinner and rougher, and the cladding mode resonances fully collapse at a thickness of $18 \mathrm{~nm}$ and roughness of $14 \mathrm{~nm}$. Upon further etching the resonances reappear and the transmission spectra approaches that of a bare TFBG immersed in the water solution. At one special point, identified by a complete collapse of the resonances (i.e. at percolation), the silver film provides full impedance matching for cladding modes between glass and the water solution at near infrared wavelengths, since the spectra is then identical to that of a bare TFBG immersed in index matching liquid, so the coating then behaves as an antireflectionlayer.
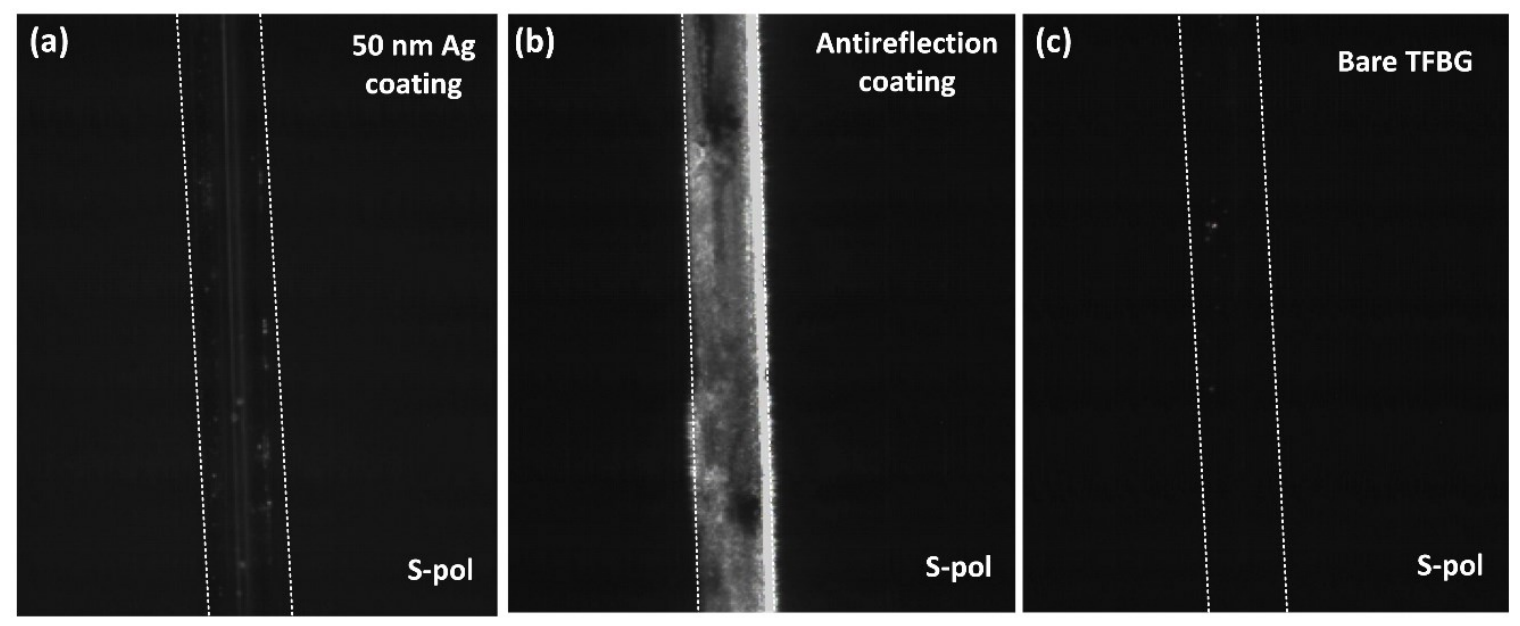

Fig. 5.2. Far-field scattering from the metal surface of a TFBG pumped by broadband Spolarized light and immersed in water for three thicknesses during the etching of the metal: (a) $50 \mathrm{~nm}$; (b) $18 \mathrm{~nm}$; (c) $0 \mathrm{~nm}$ (bare fiber grating).

Fig. 5.2 shows the far-field scattering of metal surface during etching. As we expect the scattering is weak for $50 \mathrm{~nm}$ coated Ag film because the evanescent field of cladding modes is shielded by the "thick" metal film, but we still can "see" some scattering light because of small roughness in the metal surface. On the other hand, the scattered light at the antireflection film thickness is very bright (comparatively) resulting from the fact that 
the full impedance matching for cladding modes between glass and the water makes the resonances collapse and extracts the light transferred from the core to the cladding directly through the metal surface, leading to strong scattering. The last image (all images were captured with the same settings of input power and camera sensitivity) further demonstrates that no such scattering occurs in bare fiber grating immersed in water as all the light remains confined by total internal reflection in the cladding.

In the future, we will focus on three main issues: firstly, measure the far-field scattering of a bare fiber grating immersed in refractive index matched liquid when all cladding modes collapse, which will further support the antireflection theory of the $\mathrm{Ag}$ coating at percolation. Secondly, measure the far-field scattering from the metal surface through P- or S-polarized cladding mode in real time and separately during the gradual etching process, and analyse the correspondence between the scattering properties for the two polarization states during the change from metal to insulator. Finally, measure the nonlinear properties of antireflection coated fiber grating by using high peak power pulse laser, which would lead to new applications such as nonlinear ultrafast switching or frequency conversion.

\section{References}

1. B. Gompf, J. Beister, T. Brandt, J. Pflaum, and M. Dressel, "Nanometer-thick Au-films as antireflection coating for infrared light," Opt. Lett. 32, 1578-1580 (2007).

2. J. Kroll, J. Darmo, and K. Unterrainer, "Metallic wave-impedance matching layers for broadband terahertz optical systems," Opt. Express 15(11), 6552-6560 (2007).

3. A. Thoman, A. Kern, H. Helm, and M. Walther, "Nanostructured gold films as broadband terahertz antireflection coatings," Physical Review B 77, 195405 (2008). 


\section{Chapter 6: Conclusion}

The primary focus of this thesis has been setting on all optical switching based on lightmatter interaction between the evanescent field of the cladding mode of TFBG and surface coated material such as the $\mathrm{Ag}$ film at percolation, graphene, Au metal film with surface plasmon excitation.

The TFBG, inscribed in standard telecom single mode fiber without modification of fiber by using phase mask technology, couples the forward propagation core mode to hundreds of backward propagation discrete cladding mode (i.e. $0.2-2 \mathrm{~nm}$ separated) with high Q factor (larger than $\left.10^{4}\right)$ ). An important feature of TFBGs is that the evanescent field of the cladding modes can be oriented either parallel or perpendicular to the cladding surface by linearly polarizing the input core light in S- or P-polarization relative to the tilt plane. Upon any change in the external medium permittivity, the narrow cladding resonances shift in wavelength and/or amplitude, resulting in large transmission level modifications within their spectral width, and hence it is used as a light-matter interaction platform for the rest of work during my PHD.

Firstly, the occurrence of percolation threshold by slowly etched $50 \mathrm{~nm}$ thick silver films deposited on TFBGs has been detected in-situ and in real time through the TFBG transmission spectra. The S polarized cladding modes (i.e. azimuthally polarized electric fields aligned in the plane of the coating layer) show a sudden and very large change in the wavelengths (by more than $1 \mathrm{~nm}$ ) and amplitudes (by more than $10 \mathrm{~dB}$ ). It was verified by simultaneous measurements of the electrical conductivity of the silver coating during etching, convincing the beginning of a loss of conductivity when the percolation threshold happens. In contrast to other methods to prepare thin metal films exactly at the percolation 
threshold by measuring their thickness, TFBG measurements can precisely determine the moment where the layer goes through a metal-to-insulator (or insulator-to-metal) transition during deposition or etching instead of by trial and error using external measurements on multiple samples.

Secondly, an all single-mode fiber configuration for photo-thermal based bistability and self-pulsing has been investigated by using a multi-layered graphene coated TFBG and single watt level CW laser pumping. Self-starting pulsing at a rate of up to $269 \mathrm{~Hz}$, pulse width of $1.4 \mathrm{~ms}$, and modulation amplitudes larger than $50 \%$ has been observed, corresponding to $80 \mu \mathrm{W}$ inside the graphene layer (i.e. energy density of $29 \mathrm{~kW} / \mathrm{cm}^{2}$ ) with over of $1 \mathrm{~W}$ continuous wavelength light in the fiber core, in which the oscillation threshold could be lowered through using the fibers with thinner cladding resulting in higher intensity mode fields inside the graphene layer located on the surface fiber. The bistability arises from the modulation of the transmitted spectra cladding resonance (i.e, wavelength shift and amplitude attenuation) via the rapid heating of the graphene layer. This demonstrated configuration can potentially be extended to any other system where the power coupled to the cladding of a TFBG would cause a change in the effective index of the cladding mode resonances, including coating materials with large nonlinear optical coefficients to change refractive indices through the much faster Kerr effect.

Thirdly, an all optical synchronous modulation of a CW signal propagating in the standard single fiber core by $4.5 \%$, with a modulating efficiency of $30 \pm 7 \%$ per $\mathrm{GW} / \mathrm{cm}^{2}$ and pulse duration widening of $\sim 30$ ps was achieved by core-pumping a gold-coated TFBG in water with 25 ps pulses of near infrared light at $1 \mathrm{MHZ}$ and $50 \mathrm{~mW}$ of average input power. The switching arises from modification of metal complex permittivity on a time 
scale of the order of picoseconds and hence modulate the cladding mode resonance wavelengths and amplitude of the TFBG because of hot carriers generation from the SPPs on the interface of Au film and water together with taking advantage of low insertion, the dense comb of narrowband cladding mode resonances with high $\mathrm{Q}$ factor, and the strong light-matter interaction efficiency of the plasmonic hybridized cladding modes, leading to further potential applications in fiber-based nonlinear light-matter interactions.

Finally, broadband antireflection of cladding modes based on partially etched Ag film at percolation coated on the surface of fiber grating was achieved by gradually etching of $50 \mathrm{~nm}$ thick RF coated Ag film, in which the scattering light at antireflection film surface in near-infrared wavelength region is strong (i.e. working as a photonic lighthouse), resulting from the fact that the full impedance matching for cladding modes between glass and the water makes the resonance collapse and extracts the light guided inside the fiber to metal surface, leading to strong scattering. The potential application in terms of nonlinear scattering by high peak power pumping through fiber core will be continued in future work. 